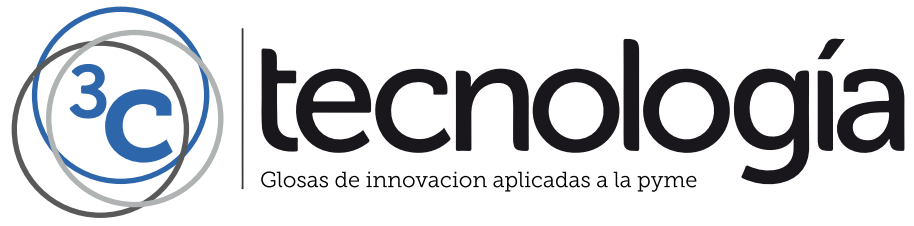

Edición Especial Marzo 2020

Special Issue March 2020

ISSN: $2254-4143$

KALASALINGAM GLOBAL CONFERENCE (KGC-2019)

INTERNATIONAL CONFERENCE ON SUSTAINABLE DEVELOPMENT

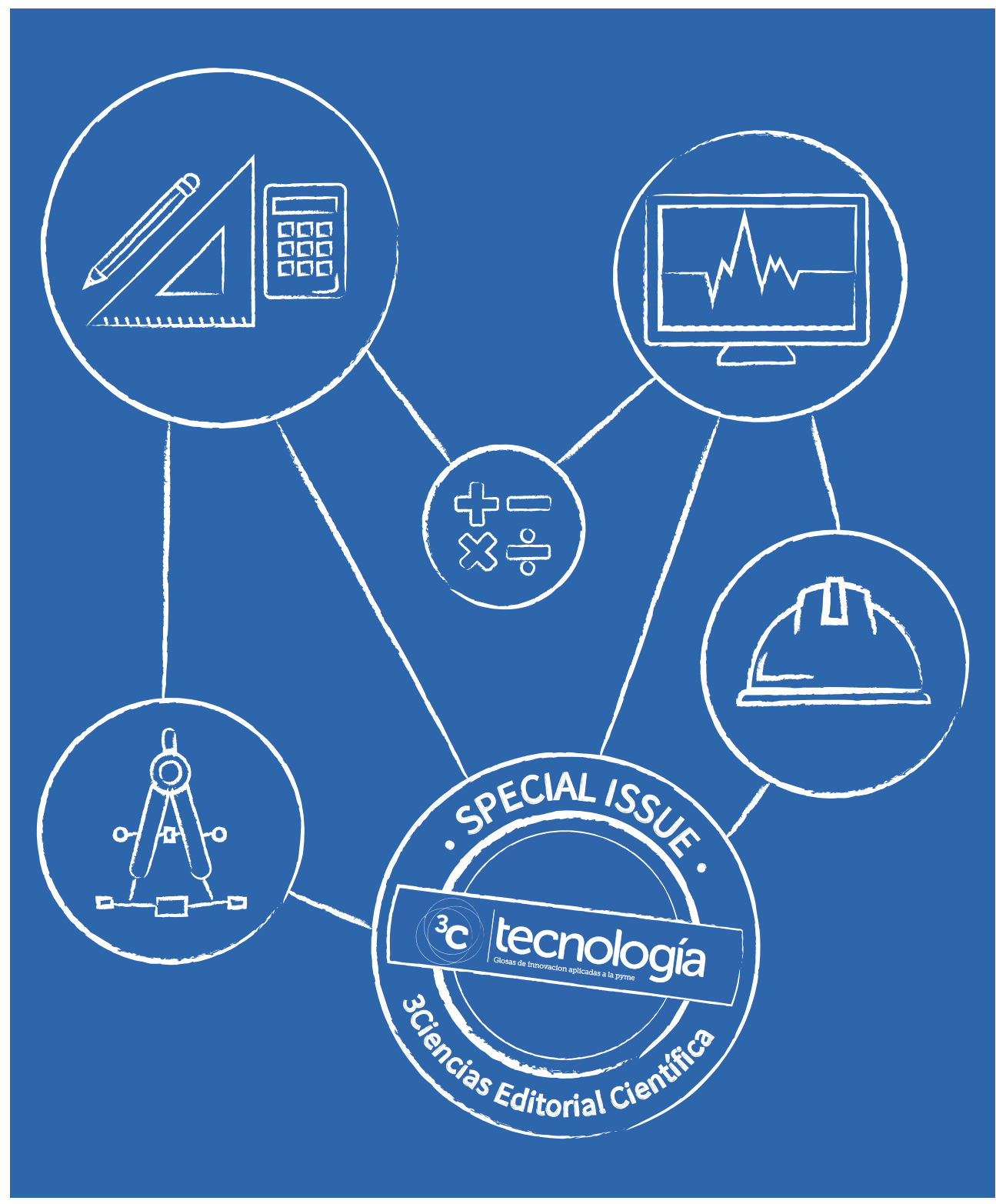


"Kalasalingam Global Conference (KGG-2019). International Conference on Sustainable Development"

\section{C Tecnología. Glosas de innovación aplicadas a la pyme.}

Edición Especial. Marzo 2020. Special Issue. March 2020.

Tirada nacional e internacional. National and internacional circulation.

Artículos revisados por el método de evaluación de pares de doble ciego.

Articles reviewed by the double blind peer evaluation method.

ISSN: $2254-4143$

No de Depósito Legal: A $268-2012$

DOI: http://doi.org/10.17993/3ctecno.2020.specialissue4

Edita:

Área de Innovación y Desarrollo, S.L.

C/Alzamora 17, Alcoy, Alicante (España)

Tel: 965030572

info@3ciencias.com _www.3ciencias.com

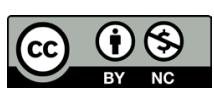

Todos los derechos reservados. Se autoriza la reproducción total o parcial de los artículos citando la fuente y el autor.

This publication may be reproduced by mentioning the source and the authors.

Copyright (C) Área de Innovación y Desarrollo, S.L. 


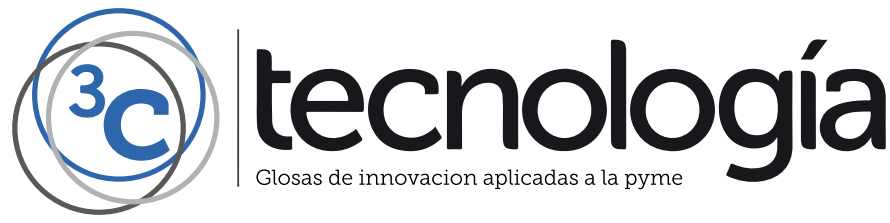




\section{CONSEJO EDITORIAL EDITORIAL BOARD}

Director

Editores adjuntos

Editores asociados
Víctor Gisbert Soler

María J. Vilaplana Aparicio

Maria Vela Garcia

David Juárez Varón

F. Javier Cárcel Carrasco

\section{CONSEJO DE REDACCIÓN DRAFTING BOARD}

Dr. David Juárez Varón. Universidad Politécnica de Valencia (España)

Dr. Martín León Santiesteban. Universidad Autónoma de Occidente (México)

Dr. F. Javier Cárcel Carrasco. Universidad Politécnica de Valencia (España)

Dr. Alberto Rodríguez Rodríguez. Universidad Estatal del Sur de Manabi (Ecuador)

\section{CONSEJO ASESOR ADVISORY BOARD}

Dra. Ana Isabel Pérez Molina. Universidad Politécnica de Valencia (España)

Dr. Julio C. Pino Tarragó. Universidad Estatal del Sur de Manabi (Ecuador)

Dr. Jorge Francisco Bernal Peralta. Universidad de Tarapacá (Chile)

Dr. Roberth O. Zambrano Santos. Instituto Tecnológico Superior de Portoviejo (Ecuador)

Dr. Sebastián Sánchez Castillo. Universidad de Valencia (España)

Dra. Sonia P. Ubillús Saltos. Instituto Tecnológico Superior de Portoviejo (Ecuador)

Dr. Jorge Alejandro Silva Rodríguez de San Miguel. Instituto Politécnico Nacional (México) 


\section{CONSEJO EDITORIAL EDITORIAL BOARD}

Área téxtil

Área financiera

Organización de empresas y RRHH

Estadística; Investigación operativa

Economía y empresariales

Sociología y Ciencias Políticas

Derecho

Ingeniería y Tecnología

Tecnologías de la Información y la Comunicación

Ciencias de la salud
Dr. Josep Valldeperas Morell

Universidad Politécnica de Cataluña (España)

Dr. Juan Ángel Lafuente Luengo

Universidad faime I (España)

Dr. Francisco Llopis Vañó

Universidad de Alicante (España)

Dra. Elena Pérez Bernabeu

Universidad Politécnica de Valencia (España)

Dr. José Joaquín García Gómez

Universidad de Almería (España)

Dr. Rodrigo Martínez Béjar

Universidad de Murcia (España)

Dra. María del Carmen Pastor Sempere

Universidad de Alicante (España)

Dr. David Juárez Varón

Universidad Politécnica de Valencia (España)

Dr. Manuel Llorca Alcón

Universidad Politécnica de Valencia (España)

Dra. Mar Arlandis Domingo

Hospital San fuan de Alicante (España) 


\section{POLÍTICA EDITORIAL}

\section{OBJETIVO EDITORIAL}

La Editorial científica 3Ciencias pretende transmitir a la sociedad ideas y proyectos innovadores, plasmados, o bien en artículos originales sometidos a revisión por expertos, o bien en los libros publicados con la más alta calidad científica y técnica.

\section{COBERTURA TEMÁTICA}

3C Tecnología es una revista de carácter científico-social en la que se difunden trabajos originales que abarcan la Arquitectura y los diferentes campos de la Ingeniería, como puede ser Ingeniería Mecánica, Industrial, Informática, Eléctrica, Agronómica, Naval, Física, Química, Civil, Electrónica, Forestal, Aeronáutica y de las Telecomunicaciones.

\section{NUESTRO PÚBLICO}

- Personal investigador.

- Doctorandos.

- Profesores de universidad.

- Oficinas de transferencia de resultados de investigación (OTRI).

- Empresas que desarrollan labor investigadora y quieran publicar alguno de sus estudios. 


\section{AIMS AND SCOPE}

\section{PUBLISHING GOAL}

3Ciencias wants to transmit to society innovative projects and ideas. This goal is reached thought the publication of original articles which are subdue to peer review or thorough the publication of scientific books.

\section{TEMATIC COVERAGE}

3C Tecnología is a scientific-social journal in which original works that cover Architecture and the different fields of Engineering are disseminated, such as Mechanical, Industrial, Computer, Electrical, Agronomic, Naval, Physics, Chemistry, Civil, Electronics, Forestry, Aeronautics and Telecommunications.

\section{OUR TARGET}

- Research staff.

- PhD students.

- Professors.

- Research Results Transfer Office.

- Companies that develop research and want to publish some of their works. 


\section{NORMAS DE PUBLICACIÓN}

3C Tecnología es una revista arbitrada que utiliza el sistema de revisión por pares de doble ciego (double-blind peer review), donde expertos externos en la materia sobre la que trata un trabajo lo evalúan, siempre manteniendo el anonimato, tanto de los autores como de los revisores. La revista sigue las normas de publicación de la APA (American Psychological Association) para su indización en las principales bases de datos internacionales.

Cada número de la revista se edita en versión electrónica (e-ISSN: 2254 - 4143), identificándose cada trabajo con su respectivo código DOI (Digital Object Identifier System).

\section{PRESENTACIÓN TRABAJOS}

Los artículos se presentarán en tipo de letra Baskerville, cuerpo 11, justificados y sin tabuladores. Han de tener formato Word. La extensión será de no más de 6.000 palabras de texto, incluidas referencias.

Los trabajos deben ser enviados exclusivamente por plataforma de gestión de manuscritos OJS:

\section{https://ojs.3ciencias.com/}

Toda la información, así como las plantillas a las que deben ceñirse los trabajos se encuentran en:

https://www.3ciencias.com/normas-de-publicacion/ 


\section{SUBMISSION GUIDELINES}

3C Tecnología is an arbitrated journal that uses the double-blind peer review system, where external experts in the field on which a paper deals evaluate it, always maintaining the anonymity of both the authors and of the reviewers. The journal follows the standards of publication of the APA (American Psychological Association) for indexing in the main international databases.

Each issue of the journal is published in electronic version (e-ISSN: $2254-4143$ ), each work being identified with its respective DOI (Digital Object Identifier System) code.

\section{PRESENTATION WORK}

The papers will be presented in Baskerville typeface, body 11, justified and without tabs. They must have Word format. The extension will be no more than 6.000 words of text, including references.

Papers must be submitted exclusively by OJS manuscript management platform: https://ojs.3ciencias.com/

All the information, as well as the templates to which the works must adhere, can be found at: https://www.3ciencias.com/normas-de-publicacion/ 


\section{ESTRUCTURA}

Los trabajos originales tenderán a respetar la siguiente estructura: introducción, métodos, resultados, discusión/conclusiones, notas, agradecimientos y referencias bibliográficas.

Es obligatoria la inclusión de referencias, mientras que notas y agradecimientos son opcionales. Se valorará la correcta citación conforme a la 7. a edición de las normas APA.

\section{RESPONSABILIDADES ÉTICAS}

No se acepta material previamente publicado (deben ser trabajos inéditos). En la lista de autores firmantes deben figurar única y exclusivamente aquellas personas que hayan contribuido intelectualmente (autoría), con un máximo de 4 autores por trabajo. No se aceptan artículos que no cumplan estrictamente las normas.

\section{INFORMACIÓN ESTADÍSTICA SOBRE TASAS DE ACEPTACIÓN E INTERNA- CIONALIZACIÓN}

- Número de trabajos aceptados publicados: 20.

- Nivel de aceptación de manuscritos en este número: 76,92\%.

- Nivel de rechazo de manuscritos: $23,08 \%$.

- Internacionalización de autores: 1 país (India).

Normas de publicación:

https://www.3ciencias.com/normas-de-publicacion/instrucciones/ 


\section{STRUCTURE}

The original works will tend to respect the following structure: introduction, methods, results, discussion/conclusions, notes, acknowledgments and bibliographical references.

The inclusion of references is mandatory, while notes and acknowledgments are optional. The correct citation will be assessed according to the 7 th edition of the APA standards.

\section{ETHICAL RESPONSIBILITIES}

Previously published material is not accepted (they must be unpublished works). The list of signatory authors should include only and exclusively those who have contributed intellectually (authorship), with a maximum of 4 authors per work. Articles that do not strictly comply with the standards are not accepted.

\section{STATISTICAL INFORMATION ON ACCEPTANCE AND INTERNATIONALI- ZATION FEES}

- Number of accepted papers published: 20.

- Level of acceptance of manuscripts in this number: 76,92\%.

- Level of rejection of manuscripts: $23,08 \%$.

- Internationalization of authors: 1 country (India).

Guidelines for authors:

https://www.3ciencias.com/en/regulations/instructions/ 


\section{INDEXACIONES INDEXATIONS}
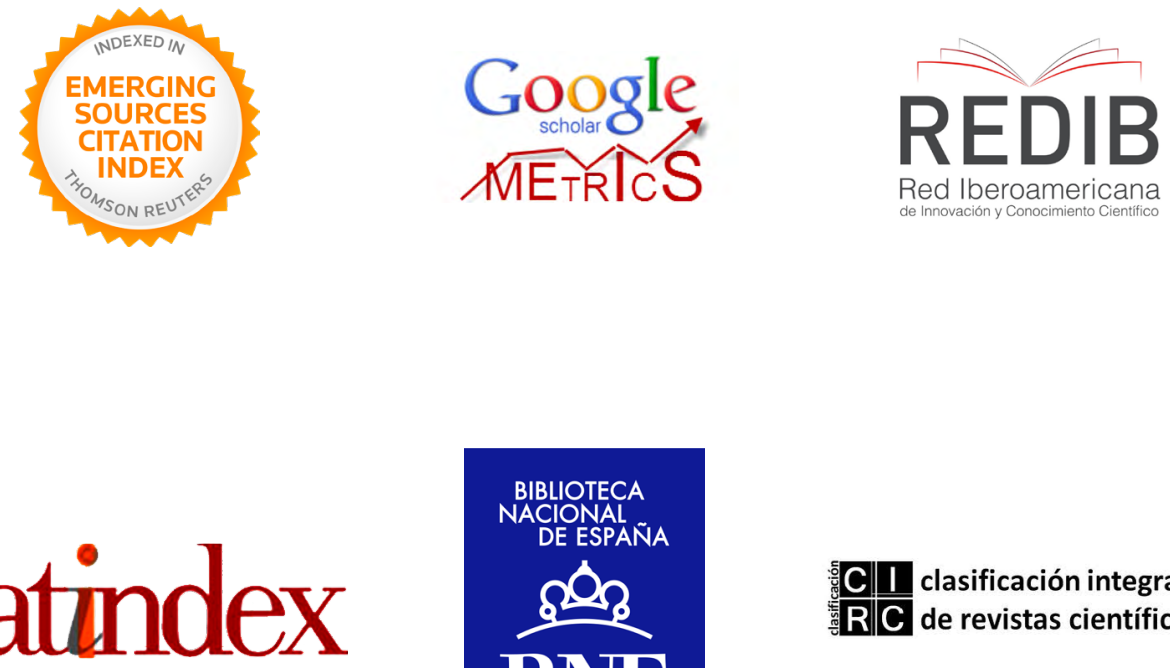

Red Iberoamericana

BIBLIOTECA

NACIONAL

DE ESPAÑA

latindex 


\section{INDEXACIONES INDEXATIONS}

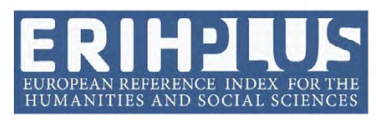

\section{- Dialnet}

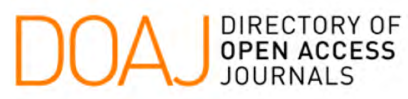

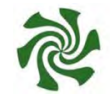

DULCINEA

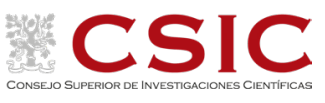

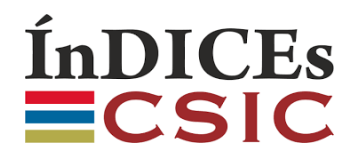

STWorldCat" 

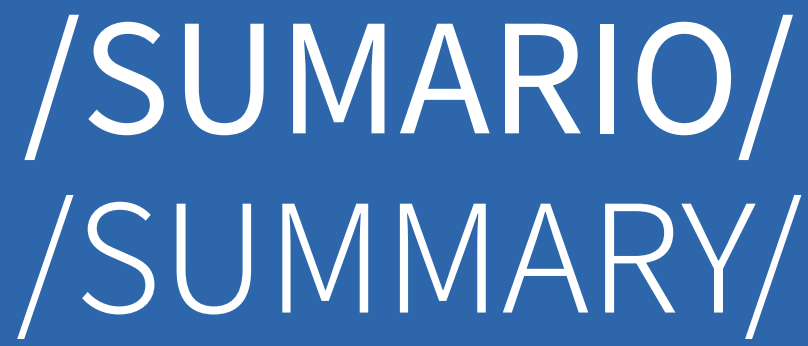
Test Time Optimization by Revisiting Notes in VLSI BIST Technique

H. Sribhuvaneshwari \& K. Suthendran

Dual biometric encrypted authentication using Rasperry Pi processor

Sivasankari Narasimhan \& Muthukumar Arunachalam

Secured Transmission in Double Clustered Heterogeneous Mobile Wireless Sensor

Network

T. Preethiya, A. Muthukumar \& S. Durairaj

Balking and reneging of batches in vod applications

R. Vanalakshmi, S. Maragathasundari \& K. S. Dhanalakshmi

A study on stages of queuing system in aircraft control system

S. Maragathasundari, C. Prabhu \& M. Palanivel

Design and optimization of reversible look ahead carry adder and carry save adder

N. Bhuvaneswar \& A. Lakshmi

Enhancing underwater images using piecewise linear smoothing gradient guided filter

A. Chrispin Jiji \& N. Ramrao

The human ear recognition based on phase-based matching algorithm

M. Muthukumar Arunachalam

New Intuition on Ear Authentication with Gabor Filter Using Fuzzy Vault

A. Kavipriya \& M. Arunachalam

Mememtic algorithm based on hill climbing algorithm for IC partitioning

K. Jeya Prakash \& P. Sivakumar

Modified Sobel Mask to Locate Knee Joint Boundaries

S. Sheik Abdullah \& M. Pallikonda Rajasekaran

Human 2D Ear Biometric Recognition Based on Contour Matching Technique 
Design of Modified March-C Algorithm and Built-in self-test architecture for Memories

G. Karthy \& P. Sivakumar

Queuing system in Synchronous Optical Network (SONET)

S. Maragathasundari, P. Suthersan \& K. S. Dhanalakshmi

Survey on various perspectives of raman amplifiers

Sumathy Raju \& Muthukumar Arunachalam

Music recommendation system based on facial emotion recognition

Deny John Samuvel, B. Perumal \& Muthukumaran Elangovan

Intelligent gas booking and leakage system using wireless sensor networks

Kalpana Murugam

Smart driving system with automatic driver alert and braking mechanism

P. B. Dhanusha, A. Lakshmi \& K. Saravanan

Implementation of differential evolution algorithm to perform image fusion for identifying brain tumor

Pothiraj Sivakumar, Subbiah Parvathy Velmurugan \& Jenyfal Sampson

Optimal choice of supervised techniques for MR image classification

Balasubramanian Aruna Devi \& Murugan Pallikonda Rajasekaran 


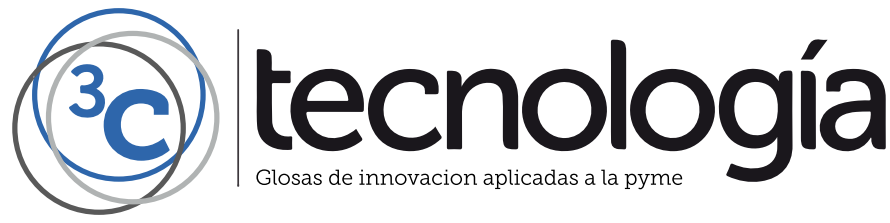




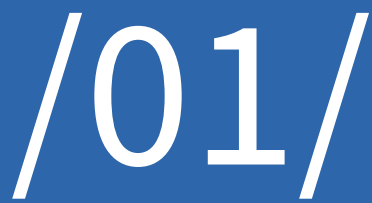




\section{TEST TIME OPTIMIZATION BY REVISITING NOTES IN VLSI BIST TECHNIQUE}

H. Sribhuvaneshwari

Research scholar, ECE Department, Kalasalingam Academy of Research and Education,

Krishnankoil, Tamilnadu, (India).

E-mail: havisriece@gmail.com ORCID: https://orcid.org/0000-0002-4804-4171

K. Suthendran

HoD/ IT Department, Kalasalingam Academy of Research and Education,

Krishnankoil, Tamilnadu, (India).

E-mail: k.suthendran@klu.ac.in ORCID: https://orcid.org/0000-0002-7030-4398

Recepción: 05/12/2019 Aceptación: 08/01/2020 Publicación: 23/03/2020

\section{Citación sugerida:}

Sribhuvaneshwari, H., y Suthendran, K. (2020). Test Time Optimization by Revisiting Notes in VLSI BIST Technique. 3 C Tecnología. Glosas de innovación aplicadas a la pyme. Edición Especial, Marzo 2020, 19-33. http://doi.org/10.17993/3ctecno.2020.specialissue4.19-33

\section{Suggested citation:}

Sribhuvaneshwari, H., \& Suthendran, K. (2020). Test Time Optimization by Revisiting Notes in VLSI BIST Technique. 3C Tecnología. Glosas de innovación aplicadas a la pyme. Edición Especial, Marzo 2020, 19-33. http://doi.org/10.17993/3ctecno.2020.specialissue4.19-33 


\section{ABSTRACT}

An effective method for test time minimization in Built In Self Test (BIST) using graph theory concept with revisiting of node is incorporated in this article. Here the shortest Hamiltonian path of ISCAS89 benchmark circuit s396 is taken as an example. Minimum spanning tree with revisiting nodes is applied for s386 circuit that optimizes the time cycle for testing. Result shows that minimum spanning tree with revisiting the nodes will reduce the time cycle without compromising the test quality. Hence an effective testing is achieved using graphical approach.

\section{KEYWORDS}

BIST, Shortest Hamiltonian path, Revisiting node, Test time. 


\section{INTRODUCTION}

In this super fast technical generation the growth of technology is massive in both technical and product aspect. Testing is an essential part which deals with the quality of the product before a microelectronic product is launched in the market where BIST is a testing scheme that is capable of finding faults in integrated circuits (ICs) to make faster testing at lessexpensive with low power constraints (Girard, Nicolici, \& Wen, 2010). It plays a vital role in electronic industry because a device that needs to be tested at higher level (levels being: Chip - board - system - system in field) costs 10 time (and possibly more) that of cost of testing it a lower level. Digital testing is declared as testing a digital circuit to validate that it performs the particular logic functions and in appropriate time. In case of VLSI testing, it is not of much concern as how many chips are binned as flawed; rather important is how many flawed chips are binned as normal. So, trade and industry expects "VLSI testing" is to result in an accuracy of perfect chips with its functionality. Optimized test time and scheming test power are contradictory targets and therefore optimization of testing for both attributes is challenging. This topic has been addressed in the recent literature (Nicolici \& Al-Hashimi, 2003; Sakurai \& Newton, 1990; Shanmugasundaram \& Agrawal, 2011; Shanmugasundaram \& Agrawal, 2012; Gogoi \& Kalita, 2014; Venkataramani, Sindia, \& Agrawal, 2014). The BIST vectors are speedy than ATE in terms of application time, thus follow-on improvement in test time with low power (Larsson, 2006). The test vector application time ratio between ATE and BIST is represented by " $a$ ". If $a=100$ than the application time of a vector in ATE is 100 times longer than the vector application of BIST (where $\alpha>1$ ). The total time required for test is equivalent to the addition of required number of time cycles to travel from source node to destination.

\section{PRIOR WORK}

A modern approach is introduced to minimize the test time for power constrained tests (Biswas, Das, \& Petriu, 2006; Das et al., 2008; Shanmugasundaram \& Agrawal, 2011, 2012) implements a monitor to observe the movement in the scan chain of a built-in self-test (BIST). According to the switching activity the test clock frequency varies from high to low both the parameters are inversely proportional i.e., test clock frequency raise if there is low switching activity in the scan chain else falls. This approach attains $20-50 \%$ reduction in test 
time of BIST circuits with a little area overhead. Reusable scan chains (Lai, Kung, \& Lin, 1993) and pattern overlapping (Zhou, Ye, Li, Wu, \& Ke, 2009; Bryant, 1986; Tehranipoor, Nourani, \& Chakrabarty, 2005; Chloupek, Novak, \& Jenicek, 2012; Chloupek, Novak, \& Jenicek, 2012; Alpert et al., 2018) eradicates unwanted scan chain operations using patterns that bear a resemblance to the previous pattern, so the number of scan shifting is minimized.

Hence high reduction is achieved on availability of such patterns. The single fixed order twisted ring-counter design proposed in (Tharakan \& Mathew, 2015) drops down the test application cycle with multiple programmable twisted-ring counters (PTRC). Huge number of unique test patterns based on the stipulation of reconfigurable run-time programmable multiple twisted-ring-counter is anticipated which is an on-chip test generation scheme. Spontaneous strategy is implemented in (Bhakthavatchalu, Krishnan, Vineeth, \& Devi, 2014) select the best possible seed and the quantity of the irregular test examples to be produced which reduces the testing time significantly. LFSR reseeding strategies proposed in (Kim \& Kang, 2006; Chandra \& Chakrabarty, 2003; Pathak \& Pathak, 2016) are broadly received in rationale BIST to improve fault perceptibility and abbreviate test application time for incorporated circuits.

Test comes about on ISCAS and expansive ITC circuits appear that the exhibited procedure can accomplish $100 \%$ fault scope with short test time by utilizing just $0.23-2.75 \%$ of inside nets. Test application time optimization in accumulator-based test-pattern generation is projected in (Magos, Voyiatzis, \& Tarnick, 2010; Voyiatzis, 2005, 2006, 2008; Manich, Garcia-Deiros, \& Figueras, 2007; Liang, Zhang, You, Li, \& Hosam, 2013). The problem of efficiently generating test patterns which is used in finding the shortest Hamiltonian path in an associated CUT's directed graph that results tremendously low demand for hardware.

Usage of accumulator structure is the better solution to the problem of minimizing the number of cycles needed for generating a set of deterministic test patterns in a novel test pattern generation. Further enhancement can be concentrated in terms of minimizing larger search space and the exact computation of the shortest Hamiltonian path in the testpattern graph. Revisiting nodes can reduce the test application time. 


\section{TEST PATTERN SELECTION}

All VLSI chips after the manufacturing process are applied for fault analysis, in such a case it is not possible of generating all the test vectors, at the same time different patterns detects the same fault which increases the complexity of test vector and its storage requirement. For C17 benchmark circuit the following six test patterns are high in terms of fault coverage which is shown in Table 1. These patterns are considered as node here.

$$
T=[T[1], T[2], T[3], T[4], T[5], T[6]]=T[0,11,14,17,28,31]
$$

Table 1. Test vector set of s396 circuit.

\begin{tabular}{|c|c|}
\hline Test Vector & Inputs [6:0] \\
\hline T1 & 0000101 \\
\hline T2 & 0000110 \\
\hline T3 & 0001011 \\
\hline T4 & 0001110 \\
\hline T5 & 0010101 \\
\hline T6 & 1010000 \\
\hline
\end{tabular}

The odd value sequence of $(31,3)$ is $\{0,3,6,9,12,15,18,21,24,27,30,2,5,8,11,14,17,20,23,26$ ,29,1,4,7,10,13,16,19,22,25,28,31 . Likewise it is preceded for all possible combination i.e., $(31, n)$. Here $\mathrm{n}$ is the odd numbers in-between ( 1 to 31$)$. In Table 2 decimal representation of the test vectors are given in first row, column and their location are given in $5 * 5$ matrix forms. Matrix size is equivalent to the number of test vectors in the test vector set of the concern circuit test pattern'00000' is negligible, so five test patterns are taken for calculation. $\left(2^{\mathrm{n}}+1\right) \&\left(2^{\mathrm{n}}+3\right)$ sequence i.e., $3,5,9,17 \& 5,7,11,19$ is calculated by means of Hamiltonian distance.

\section{DEFINITIONS}

Let $\mathrm{k}$ is the input to the particular circuit then $2^{\mathrm{k}}$ test vectors are required to test the circuit. Test vector set is derived by filtering the high fault coverage test vectors from the actual number of test vectors (here BIST analysis \& diagnosis tool is used filter six high fault coverage test vectors of s396 benchmark circuit). In order to avoid the problem called pattern minimization a technique is carried to compare the entire test vector set based on the fault detection ability, if many test vectors detects the same fault with one bit variation in 
the test vector sequence than that place is filled with ' $\mathrm{x}$ ', by this method here six test vectors are found as essential for ISCAS89 s396 benchmark circuit. When 128 test vectors are optimized to six test vectors then the test time effectively reduced to the minimum. For this s396 circuit 128 test vectors are required to test the circuit then six test vectors are filtered by using BISTAD tool. A test vector set $\mathrm{T}$ is given below:

$$
T=[T[1], T[2], T[3], T[4], T[5], T[6]]=T[5,6,11,14,21,88]
$$

These six test vectors are considered as node here, all odd value from 0 to 127 are taken in account to formulate the sequence. The odd value sequence of $(127,3)$ is $\{0,3,6,9,12,15$ ,18,21 ,24,27,30,33,36,39,42,45,48,51,54,57,60,63,66,69,72,75,78,81,84,87,90,93,96,99,1 02,105,108, $111,114,117,120,123,126,1,4,7,10,13,16,19,22,25,28,31,34,37,40,43,46,49,52$ ,55,58,61,64,67,70,73,76,79,82,85,88,91,94,97,100,103,106,109,112,115,118,121,124,12 7,2,5,8, 11,14,17,20,23,26,29,32,35,38,41,44,47,50,53,56,59,62,65,68,71,74,77,80,83,86,8 9,92,95,98,101,104,107,110,113,116,119,122,125\}.Likewise it is preceded for all possible combination i.e., $(127, \mathrm{n})$. Here $\mathrm{n}$ is the odd numbers in-between $(0$ to 127) because $\mathrm{k}=$ 128. In Table 2 decimal representation of the test vectors are given in first row, column and their location are given in $6 * 6$ matrix forms. Matrix size is equivalent to the number of test vectors in the test vector set of the concern circuit. In general for $\mathrm{k}$ inputs $2^{\mathrm{k}}-1$ matrix are required to derive $A_{\min }$ and $A_{\text {vec }}$ matrix. $A_{\min }$ and Avec are derived by finding the minimum values of a particular point for example all matrix value of 6 to 11 are compared and got 1 as minimum value which is taken for $\mathrm{A}_{\min }$ and the corresponding matrix value $\mathrm{A} 5$ is the $\mathrm{A}_{\text {vec }}$ value. Addend patterns are in the form of $2^{\mathrm{n}}+1$ i.e., $2^{1}+1=3,2^{2}+1=5, \ldots$ if the addend patterns are in the form of $2^{\mathrm{n}}+1$ then $3,5,9,17,33$ and 65 are its test pattern set.

\section{PROPOSED METHOD}

In this paper minimum spanning tree is introduced rather than Hamiltonian path (Hamiltonian path is a path which visits each vertex exactly once and also returns to the starting vertex) in the graphical construction of the c17 \& s386 benchmark circuit. Minimum spanning tree is a tree in a graph that spans all the vertices and total weight of a tree is minimal. Addend patterns are in the form of $2^{n}+1 \& 2^{n}+3$ are taken to compare the Hamiltonian path time and minimum spanning tree time cycles. 
Table 2. Amin of $c 17$ circuit with respect to $(2 n+1)$ test patterns.

\begin{tabular}{|c|c|c|c|c|c|}
\hline Amin & $\mathbf{1 1}$ & $\mathbf{1 4}$ & $\mathbf{1 7}$ & $\mathbf{2 8}$ & $\mathbf{3 1}$ \\
\hline $\mathbf{1 1}$ & $\mathrm{x}$ & 1 & 2 & 1 & 3 \\
\hline $\mathbf{1 4}$ & 10 & $\mathrm{x}$ & 1 & 5 & 1 \\
\hline $\mathbf{1 7}$ & 5 & 11 & $\mathrm{X}$ & 14 & 5 \\
\hline $\mathbf{2 8}$ & 5 & 1 & 4 & $\mathrm{x}$ & 1 \\
\hline
\end{tabular}

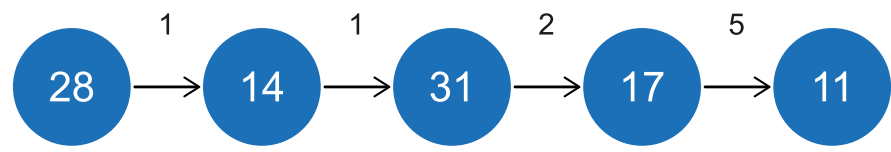

Figure 1. Hamiltonian path of $c 17$ with Addend patterns are in the form of $\left(2^{n}+1\right)$.

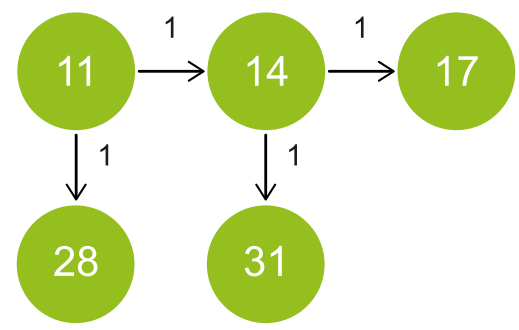

Figure 2. Minimum spanning tree of $c 17$ with Addend patterns are in the form of $\left(2^{n}+1\right)$.

The shortest Hamiltonian path for c17 circuit is $28 \rightarrow 14 \rightarrow 31 \rightarrow 17 \rightarrow 11(1,1,2,5)$ with corresponding weights and its total weight is 9 but in case of minimum spanning tree 4 time cycle are required (Figure $1 \& 2$ ). For Addend patterns are in the form of $2^{\mathrm{n}}+3$ and the Hamiltonian path through $17 \rightarrow 28 \rightarrow 11 \rightarrow 31 \rightarrow 14$ and their corresponding weights are $(1,2$, $4,3)$ totally 10 time cycles are involved whereas in minimum spanning tree with revisiting it is reduced to 7 which denotes that 14 times cycles (Figure $3 \& 4$ ) are required for testing. Table 3. Amin of c17 circuit with respect to $(2 n+3)$ test patterns.

\begin{tabular}{|c|c|c|c|c|c|}
\hline Amin & $\mathbf{1 1}$ & $\mathbf{1 4}$ & $\mathbf{1 7}$ & $\mathbf{2 8}$ & $\mathbf{3 1}$ \\
\hline $\mathbf{1 1}$ & $\mathrm{x}$ & 14 & 9 & 10 & 4 \\
\hline $\mathbf{1 4}$ & 4 & $\mathrm{x}$ & 13 & 2 & 10 \\
\hline $\mathbf{1 7}$ & 5 & 5 & $\mathrm{x}$ & 1 & 2 \\
\hline $\mathbf{2 8}$ & 2 & 10 & 4 & $\mathrm{X}$ & 13 \\
\hline $\mathbf{3 1}$ & 2 & 3 & 11 & 5 & $\mathrm{x}$ \\
\hline
\end{tabular}




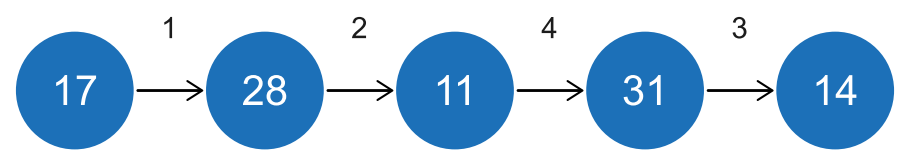

Figure 3. Hamiltonian path of $c 17$ with Addend patterns are in the form of $\left(2^{n}+3\right)$.

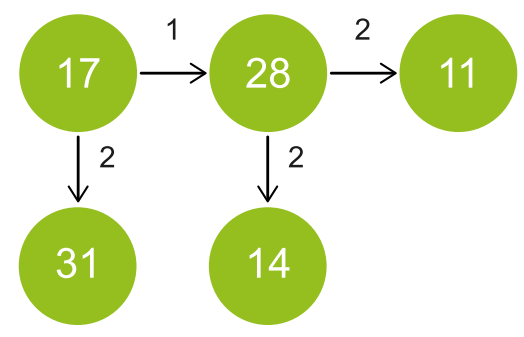

Figure 4. Minimum spanning tree of $c 17$ with Addend patterns are in the form of $\left(2^{n}+3\right)$.

Table 4. Test vector set of s396 circuit.

\begin{tabular}{|c|c|}
\hline Test Vector & Inputs [6:0] \\
\hline T1 & 0000101 \\
\hline T2 & 0000110 \\
\hline T3 & 0001011 \\
\hline T4 & 0001110 \\
\hline T5 & 0010101 \\
\hline T6 & 1010000 \\
\hline
\end{tabular}

Revisiting can reduce the testing time, here s396 benchmark circuit is taken as example which deals with 7 inputs and therefore $2^{\mathrm{k}}$ test vectors are required to test the circuit i.e., 128 test vectors. $\mathrm{A}_{\min }$ and $\mathrm{A}_{\text {vec }}$ are tabulated to derive the s396 circuit's graphical representation. All odd value sequence from 0 to 127 is taken in account for $\mathrm{A}_{\text {min }}$ and Avec calculation. The shortest Hamiltonian path for s396 circuit is $6 \rightarrow 21 \rightarrow 88 \rightarrow 5 \rightarrow 11 \rightarrow 14(1,5,3,2$, 1) with corresponding weights and its total weight is 12 but in case of minimum spanning tree 11 time cycle are required (Figure 2). Figure 3 shows the graphical representation of s386 circuit where $\mathrm{A}_{\min } \&$ Avec are derived with the consolidation of 64 matrices (all odd sequence from 0 to 127$)$. 


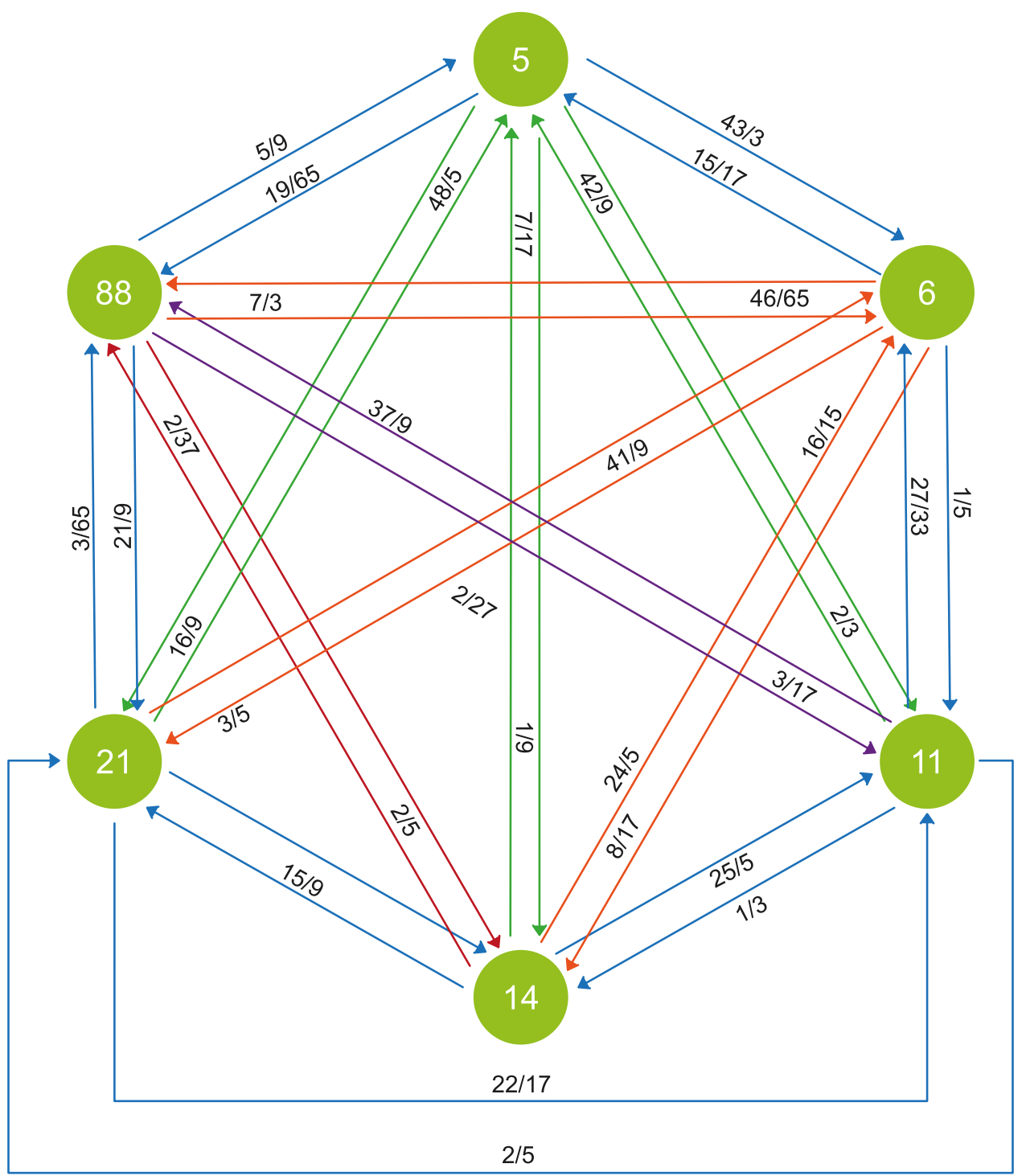

Figure 5. Graphical representation of s396 circuit.

Table 5. $A_{\min }$ of $s 386$ circuit with respect to $\left(2^{n}+1\right)$ test patterns.

\begin{tabular}{|c|c|c|c|c|c|c|}
\hline $\mathbf{A}_{\mathbf{m i n}}$ & $\mathbf{5}$ & $\mathbf{6}$ & $\mathbf{1 1}$ & $\mathbf{1 4}$ & $\mathbf{2 1}$ & $\mathbf{8 8}$ \\
\hline $\mathbf{5}$ & $\mathrm{x}$ & 43 & 2 & 1 & 16 & 19 \\
\hline $\mathbf{6}$ & 15 & $\mathrm{x}$ & 1 & 8 & 3 & 7 \\
\hline $\mathbf{1 1}$ & 42 & 27 & $\mathrm{x}$ & 1 & 2 & 37 \\
\hline $\mathbf{1 4}$ & 7 & 24 & 25 & $\mathrm{x}$ & 15 & 2 \\
\hline $\mathbf{2 1}$ & 48 & 41 & 22 & 57 & $\mathrm{x}$ & 3 \\
\hline $\mathbf{8 8}$ & 5 & 46 & 3 & 6 & 21 & $\mathrm{x}$ \\
\hline
\end{tabular}


Table 6. $A_{\min }$ of $s 386$ circuit with respect to $\left(2^{n}+3\right)$ test patterns.

\begin{tabular}{|c|c|c|c|c|c|c|}
\hline $\mathbf{A}_{\min }$ & $\mathbf{5}$ & $\mathbf{6}$ & $\mathbf{1 1}$ & $\mathbf{1 4}$ & $\mathbf{2 1}$ & $\mathbf{8 8}$ \\
\hline $\mathbf{5}$ & $\mathrm{x}$ & 11 & 2 & 53 & 48 & 17 \\
\hline $\mathbf{6}$ & 21 & $\mathrm{x}$ & 1 & 24 & 3 & 6 \\
\hline $\mathbf{1 1}$ & 46 & 73 & $\mathrm{x}$ & 33 & 2 & 7 \\
\hline $\mathbf{1 4}$ & 13 & 24 & 23 & $\mathrm{x}$ & 1 & 30 \\
\hline $\mathbf{2 1}$ & 16 & 59 & 18 & 11 & $\mathrm{x}$ & 1 \\
\hline $\mathbf{8 8}$ & 9 & 58 & 49 & 18 & 27 & $\mathrm{x}$ \\
\hline
\end{tabular}

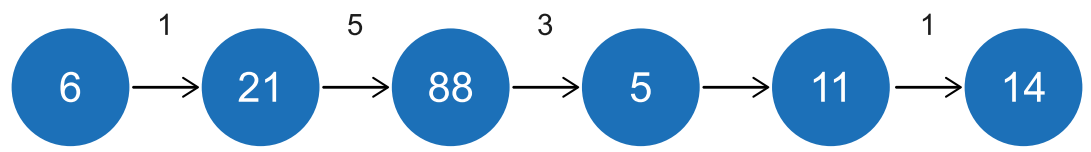

Figure 6. Hamiltonian path of $s 386$ with Addend patterns are in the form of $\left(2^{n}+1\right)$.

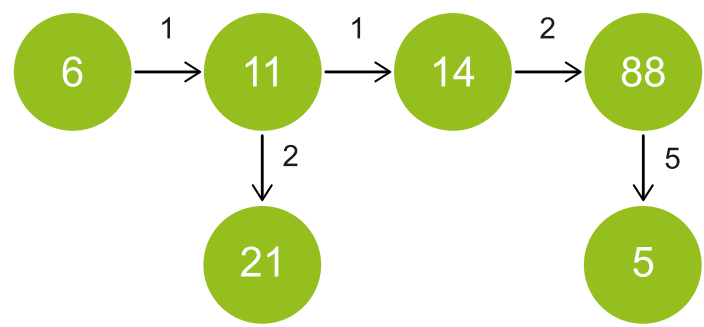

Figure 7. Minimized time spanning tree of s396 with Addend patterns are in the form of $\left(2^{n}+1\right)$.

For Addend patterns are in the form of $2^{\mathrm{n}}+3$ the Hamiltonian path through $14 \rightarrow 21 \rightarrow$ $88 \rightarrow 5 \rightarrow 6 \rightarrow 11$ and their corresponding weights are $(1,1,9,11,1)$ totally 23 time cycles are involved whereas in minimum spanning tree with revisiting it is reduced to 14 which denotes that 14 times cycles (Figure 3) are required for testing.

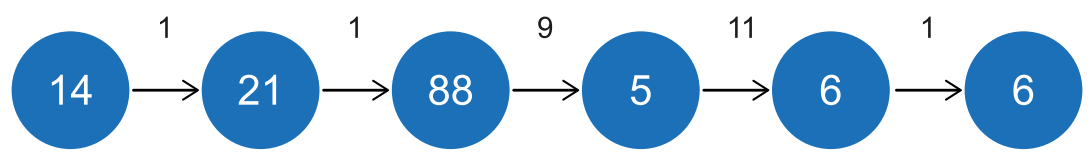

Figure 8. Hamiltonian path of $s 386$ with Addend patterns are in the form of $\left(2^{n}+3\right)$.

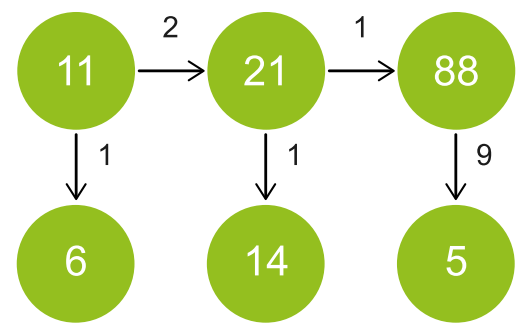

Figure 9. Minimized time spanning tree of s396 with Addend patterns are in the form of $\left(2^{n}+3\right)$. 


\section{COMPARISON}

\begin{tabular}{|c|c|c|c|}
\hline Benchmark circuit & $\begin{array}{c}\text { Addend pattern } \\
\text { form }\end{array}$ & $\begin{array}{c}\text { Hamiltonian path Time cycle } \\
\text { requirement }\end{array}$ & $\begin{array}{c}\text { Minimum spanning tree Time } \\
\text { cycle requirement }\end{array}$ \\
\hline \multirow{2}{*}{ C17 } & $2^{\mathrm{n}}+1$ & 9 & 4 \\
\hline $2^{n}+3$ & 10 & 7 \\
\hline \multirow{2}{*}{ S386 } & $2^{n}+1$ & 12 & 11 \\
\hline $2^{n}+3$ & 23 & 14 \\
\hline
\end{tabular}

Table 7. Comparison table for time cycle involvement in Hamiltonian path, minimum spanning tree with revisiting nodes.

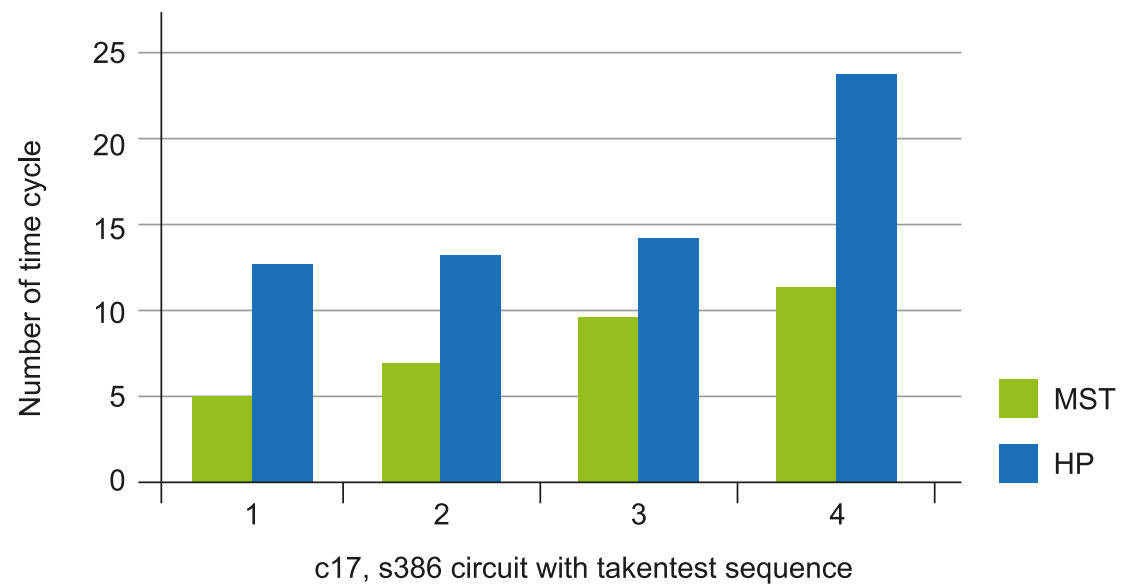

Figure 10. Graph for time cycle involvement in Hamiltonian path, minimum spanning tree with revisiting nodes.

\section{CONCLUSION}

In this paper we have presented a graph theory concept called minimum spanning tree with revisiting nodes instead of Hamiltonian path for c17 \& s396 benchmark circuit which results in optimized test time. Result shows that minimum spanning tree with revisiting the nodes will reduce the time cycle for testing. The above mentioned Table $7 \&$ Figure 10 shows that minimum spanning tree effectively reduces the number of test time cycles for testing. In future it can be implemented to test nano memories. 


\section{REFERENCES}

Alpert, G. J., Chow, W. K., Han, K., Kahng, A. B., Li, Z., Liu, D., \& Venkatesh, S. (2018, March). Prim-Dijkstra Revisited: Achieving Superior Timing-driven Routing Trees. In Proceedings of the 2018 International Symposium on Physical Design (pp. 10-17). ACM.

Bhakthavatchalu, R., Krishnan, S., Vineeth, V., \& Devi, M. N. (2014, July). Deterministic seed selection and pattern reduction in Logic BIST. In 18th International Symposium on VLSI Design and Test (pp. 1-2). IEEE.

Biswas, S., Das, S. R., \& Petriu, E. M. (2006). Space compactor design in VLSI circuits based on graph theoretic concepts. IEEE Transactions on Instrumentation and Measurement, 55(4), 1106-1118. https://doi.org/10.1109/TIM.2006.876523

Bryant, R. E. (1986). Graph-based algorithms for boolean function manipulation. IEEE Transactions on Computers, 100(8),677-691.https://doi.org/10.1109/TC.1986.1676819

Ghandra, A., \& Ghakrabarty, K. (2003). A unified approach to reduce SOC test data volume, scan power and testing time. IEEE transactions on computer-aided design of integrated circuits and systems, 22(3), 352-363. https://doi.org/10.1109/TCAD.2002.807895

Ghloupek, M., Novak, O., \& Jenicek, J. (2012, April). On test time reduction using pattern overlapping, broadcasting and on-chip decompression. In 2012 IEEE 15th International Symposium on Design and Diagnostics of Electronic Circuits ES Systems (DDECS) (pp. 300-305). IEEE.

Ghloupek, M., Novak, O., \& Jenicek, J. (2012, April). On test time reduction using pattern overlapping, broadcasting and on-chip decompression. In 2012 IEEE 15th International Symposium on Design and Diagnostics of Electronic Circuits \& Systems (DDECS) (pp. 300-305). IEEE.

Das, S. R., Hossain, A., Biswas, S., Petriu, E. M., Assaf, M. H., Jone, W. B., \& Sahinoglu, M. (2008). On a new graph theory approach to designing zero-aliasing space compressors for built-in self-testing. IEEE Transactions on Instrumentation and Measurement, 57(10), 2146-2168. https://doi.org/10.1109/TIM.2007.910004 
Flores, P., Neto, H., Chakrabarty, K., \& Marques-Silva, J. (1999, May). Test pattern generation for width compression in BIST. In ISCAS'99. Proceedings of the 1999 IEEE International Symposium on Circuits and Systems VLSI (Cat. No. 99CH36349) (Vol. 1, pp. 114-118). IEEE.

Girard, P., Nicolici, N., \& Wen, X. (Eds.). (2010). Power-aware testing and test strategies for low power devices. Springer Science \& Business Media.

Gogoi, B., \& Kalita, B. (2014). Algorithm to color a Circuit Dual Hypergraph for VLSI Circuit. International Journal of Computer Science and Information Technologies, 5(4), $5047-$ 5052. http://ijcsit.com/docs/Volume\%205/vol5issue04/ijcsit2014050447.pdf

Kim, H. S., \& Kang, S. (2006). Increasing encoding efficiency of LFSR reseeding-based test compression. IEEE Transactions on Computer-Aided Design of Integrated Circuits and Systems, 25(5), 913-917. https://doi.org/10.1109/TCAD.2005.855977

Lai, W. J., Kung, C. P., \& Lin, G. S. (1993, February). Test time reduction in scan designed circuits. In 1993 European Conference on Design Automation with the European Event in ASIC Design (pp. 489-493). IEEE.

Larsson, E. (2006). Introduction to advanced system-on-chip test design and optimization (Vol. 29). Springer Science \& Business Media.

Liang, W., Zhang, D., You, Z., Li, W., \& Hosam, O. (2013). A survey of techniques for VLSI IP protection. Information Technology Journal, 12(12), 2324-2332. https:// doi.org/10.3923/itj.2013.2324.2332

Lien, W. G., Lee, K. J., Hsieh, T. Y., \& Ghakrabarty, K. (2014). Efficient LFSR reseeding based on internal-response feedback. Fournal of Electronic Testing, 30(6), 673685. https://doi.org/10.1007/s10836-014-5482-4

Magos, D., Voyiatzis, I., \& Tarnick, S. (2010). An Accumulator-Based Test-Per-Clock Scheme. IEEE Transactions on Very Large Scale Integration (VLSI). Systems, 19(6), 1090-1094. https://doi.org/10.1109/TVLSI.2010.2043452 
Manich, S., Garcia-Deiros, L., \& Figueras, J. (2007). Minimizing test time in arithmetic test-pattern generators with constrained memory resources. IEEE Transactions on Computer-Aided Design of Integrated Circuits and Systems, 26(11), 2046-2058. https://doi. org/10.1109/TCAD.2007.906465

Nicolici, N., \& Al-Hashimi, B. (2003). Power-constrained testing of VLSI circuits (p. 178). Kluwer Academic Publishers.

Pathak, V. K., \& Pathak, V. K. (2016). Design of Input Vector Monitoring Concurrent BIST based Architecture for 4-Bit Multiplier. International Journal of Computer Applications, 153(5), 19-24. https://pdfs.semanticscholar. org/9c0e/229b71f418dabcb96b54af0e8474116c9370.pdf

Sakurai, T., \& Newton, A. R. (1990). Alpha-power law MOSFET model and its applications to CMOS inverter delay and other formulas. IEEE Journal of solid-state circuits, 25(2), 584-594. https://doi.org/10.1109/4.52187

Shanmugasundaram, P., \& Agrawal, V. D. (2011, May). Dynamic scan clock control for test time reduction maintaining peak power limit. In 29th VLSI Test Symposium (pp. 248-253). IEEE.

Shanmugasundaram, P., \& Agrawal, V. D. (2012, January). Externally tested scan circuit with built-in activity monitor and adaptive test clock. In 2012 25th International Conference on VLSI Design (pp. 448-453). IEEE.

Tehranipoor, M., Nourani, M., \& Chakrabarty, K. (2005). Nine-coded compression technique for testing embedded cores in SoCs. IEEE transactions onvery largescaleintegration (VLSI) systems, 13(6), 719-731. https://doi.org/10.1109/TVLSI.2005.844311

Tharakan, A. S., \& Mathew, B. K. (2015). Design and Implementation of an On-Chip Test Generation Scheme Based on Reconfigurable Run-Time Programmable and Multiple Twisted-Ring Counters. Procedia Computer Science, 46, 1409-1416. https:// doi.org/10.1016/j.procs.2015.02.059 
Venkataramani, P., Sindia, S., \& Agrawal, V. D. (2014). A test time theorem and its applications. Fournal of Electronic Testing, 30(2), 229-236. https://doi.org/10.1007/ s10836-014-5447-7

Voyiatzis, I. (2005). Test vector embedding into accumulator-generated sequences: A linear-time solution. IEEE Transactions on Computers, 54(4), 476-484. https://doi. org/10.1109/TC.2005.69

Voyiatzis, I. (2006, September). Accumulator-based compression in symmetric transparent RAM BIST. In International Conference on Design and Test of Integrated Systems in Nanoscale Technology, 2006. DTIS 2006. (pp. 273-278). IEEE.

Voyiatzis, I. (2008, March). On reducing aliasing in accumulator-based compaction. In 2008 3rd International Conference on Design and Technology of Integrated Systems in Nanoscale Era (pp. 1-12). IEEE.

Zhou, B., Ye, Y. Z., Li, Z. L., Wu, X. C., \& Ke, R. (2009, March). A new low power test pattern generator using a variable-length ring counter. In 2009 10th International Symposium on Quality Electronic Design (pp. 248-252). IEEE. 


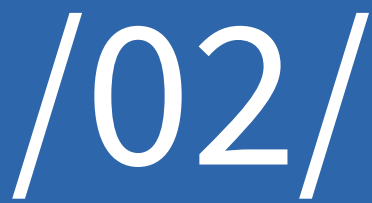




\section{DUAL BIOMETRIC ENCRYPTED AUTHENTICATION USING RASPERRY PI PROCESSOR}

Sivasankari Narasimhan

Assistant Professor, Electronics and Communication Engineering,

Mepco Schlenk Engineering College, Virudhunagar Dt, (India). E-mail: sivani.sivasankari@gmail.com ORCID: https://orcid.org/0000-0002-3162-4751

Muthukumar Arunachalam

Assistant Professor, Electronics and Communication Engineering, Kalasalingam University, Virudhunagar Dt, (India). E-mail: muthuece.eng@gmail.com ORCID: https://orcid.org/0000-0001-8070-3475

\section{Citación sugerida:}

Narasimhan, S., y Arunachalam, M. (2020). Dual biometric encrypted authentication using Rasperry PI Processor. 3C Tecnología. Glosas de innovación aplicadas a la pyme. Edición Especial, Marzo 2020, 35-49. http://doi.org/10.17993/3ctecno.2020.specialissue4.35-49

\section{Suggested citation:}

Narasimhan, S., \& Arunachalam, M. (2020). Dual biometric encrypted authentication using Rasperry PI Processor. 3C Tecnología. Glosas de innovación aplicadas a la pyme. Edición Especial, Marzo 2020, 35-49. http://doi.org/10.17993/3ctecno.2020.specialissue4.35-49 


\section{ABSTRACT}

Security is one of the main concerns in many sectors especially in banking. Many protection mechanisms such as passwords and number locks, PIN numbers have been used to identify the correct person. The biometric protection mechanism using fingerprints are also implemented. To ensure more security double biometric factors are implemented in this paper. Voice is a powerful factor to identify a speaker who is holding the account in banks. In addition to voice, usual face biometric features also considered for security in bank lockers. Both are transformed into encrypted format and stored to avoid database hacking. In this, Raspberry Pi board is used for implementation. To manipulate voice, devices like USB microphone and sound cards are used. For processing face image Raspi Cam is used. When the given image and voice matches with that of the image and voice stored in the database, then login process starts else the person trying to unlock the locker is not the bank account holder. For new users, signup process will be provided by administrator by capturing voice and face images for enrollment. This system can be helpful for maintaining the customer's confidentiality in bank lockers.

\section{KEYWORDS}

Authentication, Face recognition, Voice recognition, Encryption, Enrollment. 


\section{INTRODUCTION}

The most basic requirement of any bank locker is high security and getting high privacy regarding bank locker. Every person has precious accessories like jewelry or cash in it, so authentication of the person who wants to use the locker is very important. Effective security can be provided by using face and voice recognition biometrics. In olden days secret key is used by customers. Now-a-days customers' biometric attributes are additionally included which are unique and act as one identity for individual. A secret key can be stolen or changed. But biometric characteristics won't be changed, for example, an individual's face or voice can't be changed or imitated. The distinguished protocol for the execution of a bank locker security framework, with the authentication of human face and voice recognition, to confirm the person's character has been proposed in this paper.

The database creation phase for banking utilizes image and voice of the client to be stored using Raspberry pi. The access to open the locker is provided only to the authorized customers. If the image and the voice are not present in the database, the access permission is denied.

\section{RELATED WORKS}

Sahani, Nanda, Sahu and Pattnik (2015) proposed a remote access control framework for smart home condition. Raspberry Pi based entry to control and design home security framework through site page with ZigBee is implemented. The framework distinguishes the visitor's quality and exchanges the picture through email and SMS by GSM to already stored numbers. The client can specifically login and cooperate with the inserted gadget progressively without the need to keep up an extra server.

Baby, Munshi, Malik, Dogra and Rajesh (2017) proposed an empowering mechanism for home automation with web application for electrical apparatuses (such as fan and light) control. They are dependent on sensor inputs to indicate movements and temperature. The lock can be controlled by giving voice directions. Thus, utilizing this framework, it is currently progressively advantageous to control the machines in homes. 
Kaur, Sharma, Jain and Raj (2016) proposed an automation system using voice. With voice as information, the system interprets or follows the importance of that input and creates a proper voice yield. Utilizing voice as information, it tends to be changed over to content. This work experiences the disadvantage that just predefined voices are feasible, and it can store just restricted voices. Subsequently, the client can't get the full data.

Senthilkumar, Gopalakrishnan and Sathish Kumar (2014) wished-for image capturing system based on Raspberry Pi. Face acknowledgment is the principal concern and has the least false acknowledgment rate. The structured stage gains the pictures and stores them into the ongoing database, which is later utilized for contrasting the principles of the clients.

Shah, Patel and Patel (2018) develops a model for storing the data in computers using Rasperry PI. It can be programmed with languages like JAVA, HTML, .NET, Python in it. Rasperry PI and digital signal controller (DSC) is designed for monitoring multiple parameters based on Ethernet.

Ramani, Selvaraju, Valarmathy and Niranjan (2012) projected a secure bank locker system based on RFID and GSM. In this framework, true individual can recover cash from bank locker. This is used to approve the client and open the entryway continuously for bank locker secure access. This is more secure than different frameworks. The RFID examines the ID number from detached tag and send to the microcontroller, if the ID number is legitimate, at that point microcontroller send the SMS and ask for the confirmed individual portable number. The secret code is necessary to open the bank locker. If the individual sends the secret word to the microcontroller, it will check the passwords entered by the console and get verified from the cell phone. If these two passwords are coordinated, the locker will be opened else it will be stay in bolted position. This framework is more secure than different frameworks since two passwords required for confirmation.

Our project gives the following significant works:

- With face and voice recognition for accessing the bank locker account.

- Login page to unlock the locker of the bank account holder.

- Signup page for a new user. 
- $\quad$ Encrypted database for storing the voice and facial features.

The remaining sections are organized as follows: section 3 provides the proposed methods and section 4 gives the implementation results followed by conclusion in section 5 .

\section{PROPOSED METHODOLOGY}

The main module in our processor is Rasperry Pi kit which collects all details regarding biometric and customer's details. Raspberry Pi 3 is used for programming to create login and signup web pages by coding in PHP, capturing images, recording voice, creating databases for storing the necessary details, performing image encryption process and to perform voice and image recognition.

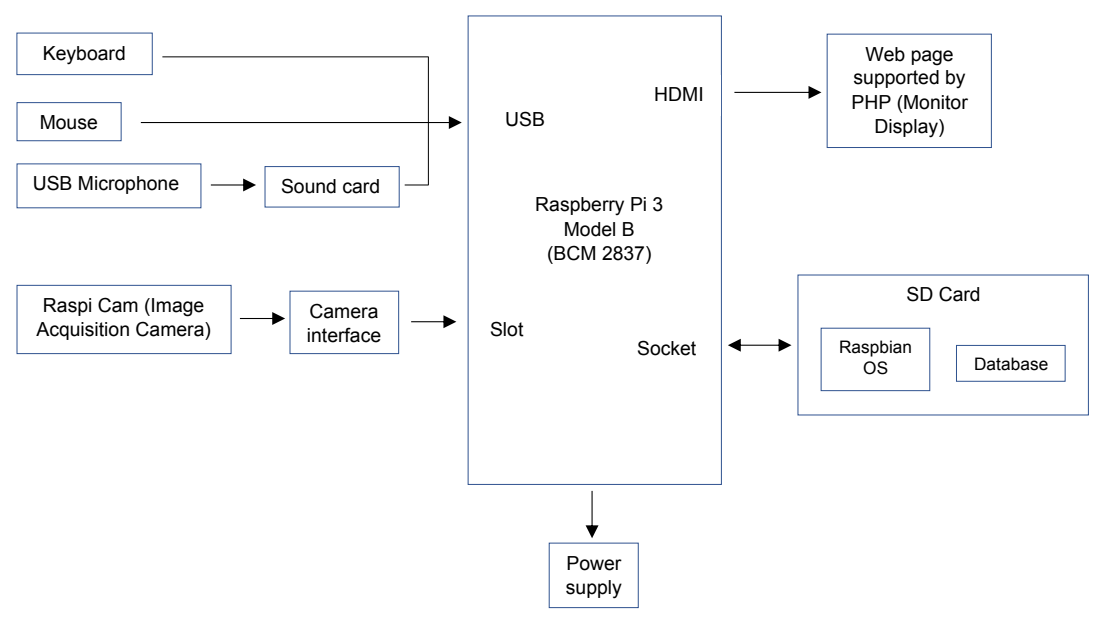

Figure 1. Overall block diagram.

Raspbian Stretch OS is used by this kit. The modules connected with Rasperry kit is shown in Figure 1. Now let us see the process involved and used components in the encrypted authentication process one by one.

\subsection{ENROLLMENT AND AUTHENTICATION PROCESS}

Bank customers account number, type of account and the persons involved in the particular ID and their facial biometric features, voice features have been collected in the process of new user enrollment. In bank database, they are stored in encrypted form. 
During login phase the customer must provide all the details to open the locker. If the details match with the database, then the locker will be opened; otherwise the person trying to open the locker is blocked by bank and alert is given to police station also. Sometimes voice features do not get matched and the facial biometrics gets matched, then there will be some likelihood that he/she may be the customer. But if face does not match, he should not be allowed to access the locker. Because face is an important feature in any individual. But voice may vary due to some unavoidable situations like cold, fever.

\subsection{FACE RECOGNITION MODULE}

Camera module captures image when capture image button is pressed in the webpage. When the button is pressed, the python code for capturing image should run. While storing that image in the database during signup, the image can be encrypted for better security. This $8 \mathrm{mp}$ camera module is equipped for 1080 pixel video and still pictures that associate straightforward to Raspberry Pi. The camera module associates with the Raspberry Pi board through the Camera Serial Interface (CSI) connector to interface with camera. The CSI transport is prepared to have high information rates, and it only conveys pixel information to the processor. The picture of Raspi camera is portrayed in Figure 2.

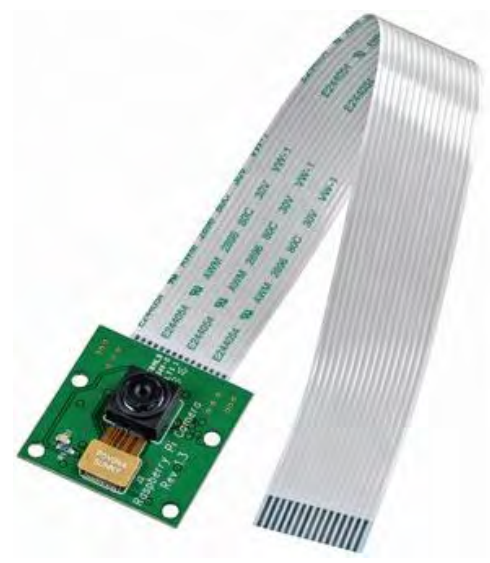

Figure 2. Raspi camera.

From the continuous pictures face image must be detected and recognized. Face detection is performed by HAAR Cascade Classifiers (Tabora, 2011). There is eye, head, and mouth and nose detectors in the HAAR cascade classifiers. Detected and processed face is compared to a database of known faces, to decide who that person is. Face Identification 
can be performed reasonably dependably, for example, with Open CV's face Identifier, working in about 90-95\% of clear photographs of an individual looking forward at the camera. The preprocessing is done to efficiently recognize the face of the customers. For that preprocessing, Eigen Face methodology concept is applied.

It is normally harder to identify an individual's face when they are seen from the side or at an edge, and occasionally this requires 3D Head Posture Estimation. Principal component analysis (PCA) is a statistical procedure that uses an orthogonal transformation to convert a set of observations of possibly correlated variables into a set of values of linearly uncorrelated variables called principal components. If the image elements are considered as random variables, the PCA basis vectors are defined as eigen vectors of the scatter matrix (ST) defined as:

$$
S_{T}=\Sigma^{M}\left(x_{i}-\mu\right) \cdot\left(x_{i}-\mu\right)^{T}
$$

where $\mu$ is the mean of all images in the training set and xi is the ith image with its columns concatenated in a vector.

\subsection{VOICE RECOGNITION MODULE}

Voice authentication is implemented in Raspberry Pi in order to add an extra layer of security. Raspberry Pi does not have a sound card and therefore it won't support microphones on audio jack, so we should use a USB microphone. Hence some additional modules are installed in Python for recording voice to perform voice recognition. The recorded voice should be of maximum 3 seconds duration. The customer can speak any of his/her secret code in their own tone. Voice recognition is done by matching the pitch of the captured speech signal and the speech stored in the database. Basic process is shown in Figure 3.

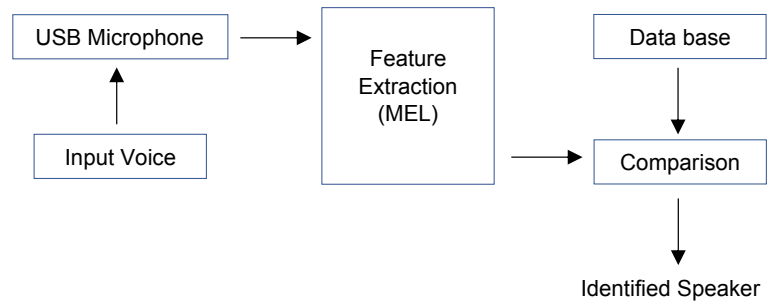

Figure 3. Voice biometric processing. 
Microphone is used to capture the voice of the customer. It is a transducer that changes over sound into electrical signal.
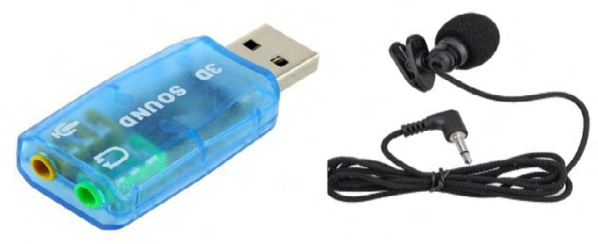

Figure 4. (a) Sound card (b) Microphone

Components used in our voice processing are shown in Figure 4. Raspberry Pi kit does not have an internal sound card. Also, the voice signal must be amplified prior to be given as input to the processor. For all these purposes an USB sound card must be used in between the USB microphone and the kit.

The sequence of steps followed in voice processing is:

- $\quad$ Frame the signal into short frames.

- $\quad$ For each frame, periodogram, power spectrum is calculated.

- Mel filter bank is applied to the power spectra.

- $\quad$ Energy is summed in each filter.

- DCT of Logarithm of all filter bank energies is taken.

- DCT coefficients 2-13 are kept and the remaining things are discarded.

In certain cases, the image may get matched, but the voice may not get matched. These cases may arise because of an individual's personal conditions. These situations are unavoidable. In such cases, the algorithm must be designed in such a manner that at these situations, the concerned person must be allowed to login by satisfying some threshold.

\subsection{ENCRYPTION}

The image obtained from RASPI camera is encrypted with AES algorithm before saving it in database. The Advanced Encryption Standard (AES) is a symmetric-key block cipher 
algorithm with Cipher Block Chaining Mode. As usual with the normal AES algorithm (Stallings, 2005) Substitute bytes, shift rows, Mix columns, Add round keys operations are taken place, Encrypted facial biometric data is stored in database.

\section{IMPLEMENTATION}

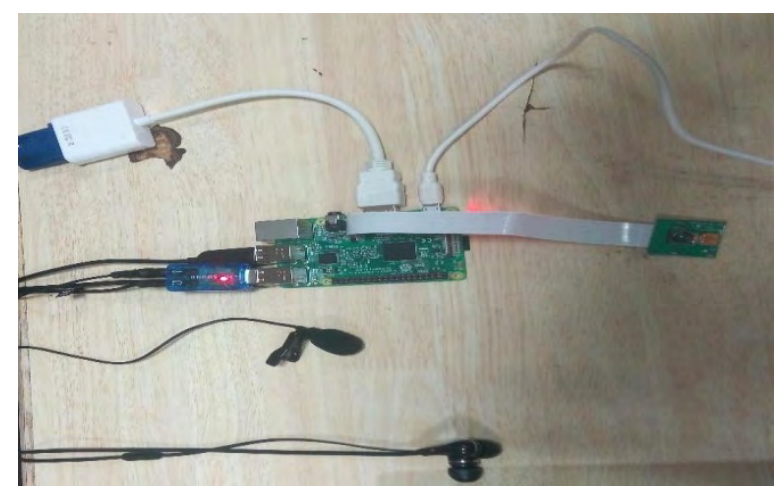

Figure 5. Hardware Setup.

Through the USB ports the keyboard, mouse and the sound card with which the microphone connection are to be made are connected. A High Definition Multimedia Interface (HDMI) to Video Graphics Array (VGA) connector is used to connect the processor to the monitor. An SD card is inserted in the slot provided at the right side. Raspi Camera module is connected to the Raspberry Pi camera interface. Hardware set up is shown in Figure 5. The face recognition module is to capture images through the Raspberry Pi camera. The images get stored in database which is created. The images shown in Figures 6(a) and 7(a) are registered face, which is stored as encrypted form as shown in Figures 6(b) and 7(b).

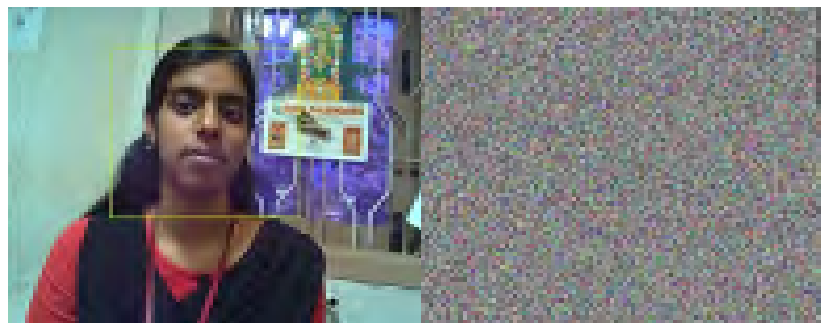

Figure 6. (a) Captured Image 1 (b) Encrypted image. 


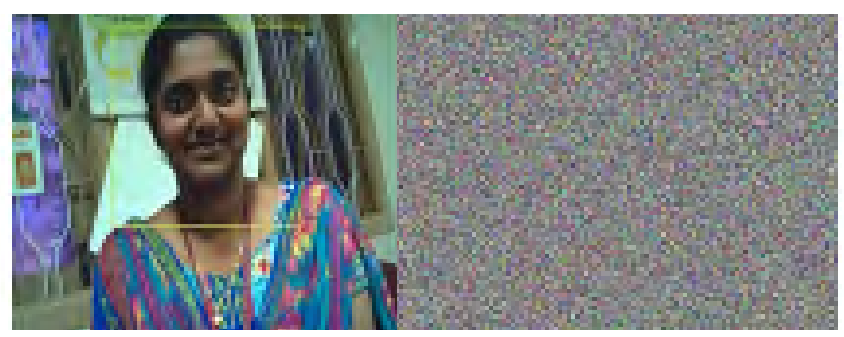

Figure 7. (a) Captured Image 2 (b) Encrypted image.

The face image from the passport size photo is located first and facial image is encrypted and stored in database. By using python coding for face recognition, first we are detecting the face and then the captured image is compared with the image that has already been captured and stored in the database. The stored images should be optimized because of the total data storage capability of the system. Since the encrypted image stores much space than the captured image, they must be compressed and then must be stored. The database contains the details of all the registered customer details. The database must be created through MySQL. The details to be stored are the customer ID, customer name, customer image and the customer voice. The sample login page created in our work is shown in Figure 9.

Voice Recognition Module: The audio signal input should be more or less 3 seconds of a wave (.wav) format file. Because for authentication due to the storage space constraints, there is limitation imposed on the length of the audio signal. That audio signal must be a code word of the customer of his own desire. The pitch values only will be compared for authentication. The sample voice images are shown in Figure 8.

Login page (shown in Figure 10) has been created for the customer to login to access his/ her bank locker if he is an already registered user. This login page asks for customer id, customer image and the customer voice. The customer image and voice are given as real time data. If the image is not registered and have customer ID and ask for authenticity means he/she will be marked as intruder. 

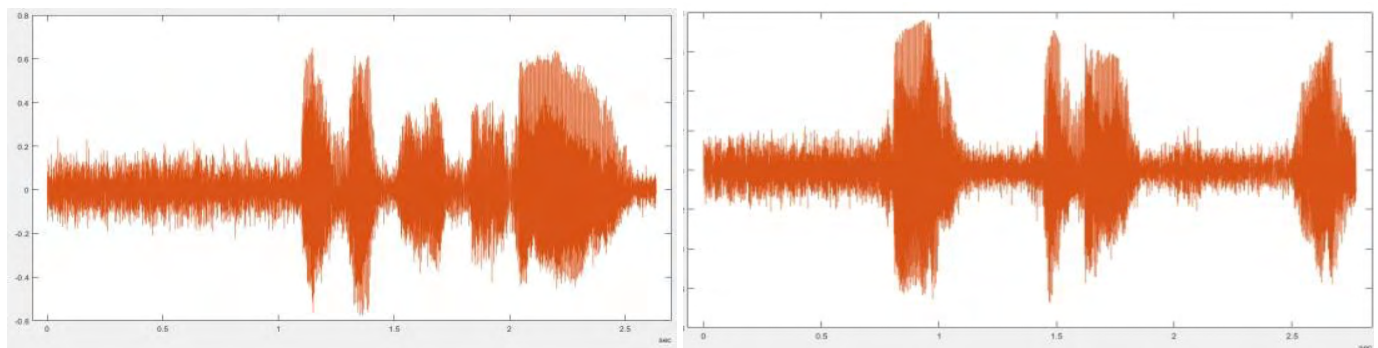

Figure 8. (a) Sample voice 1 (b) Sample voice 2.

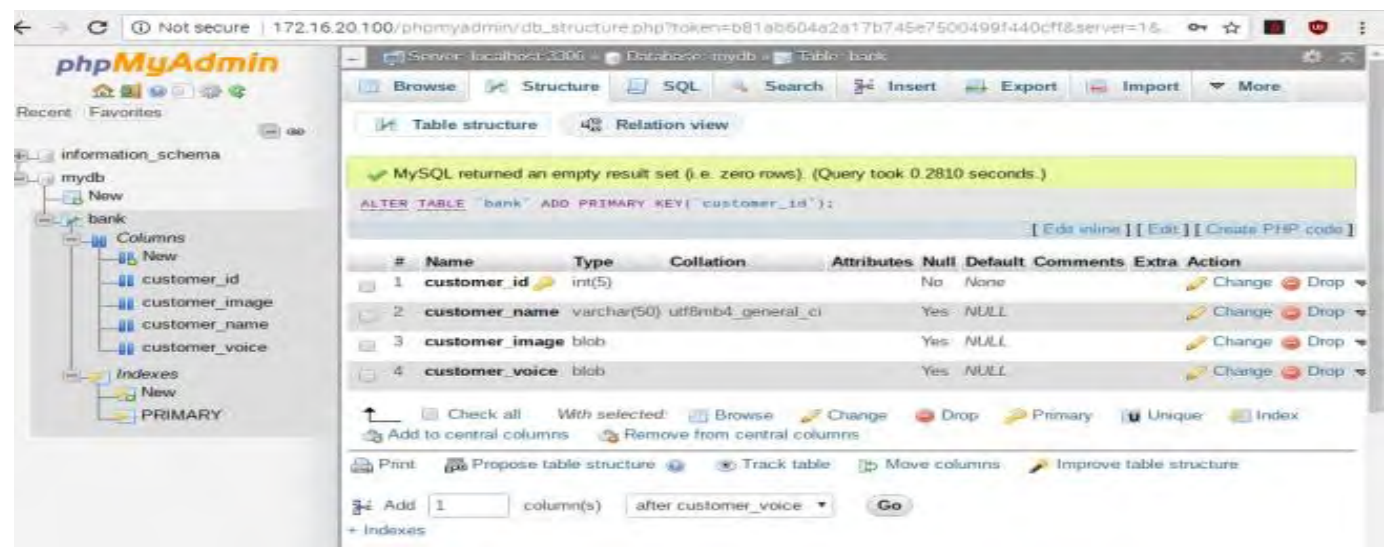

Figure 9. Database template.

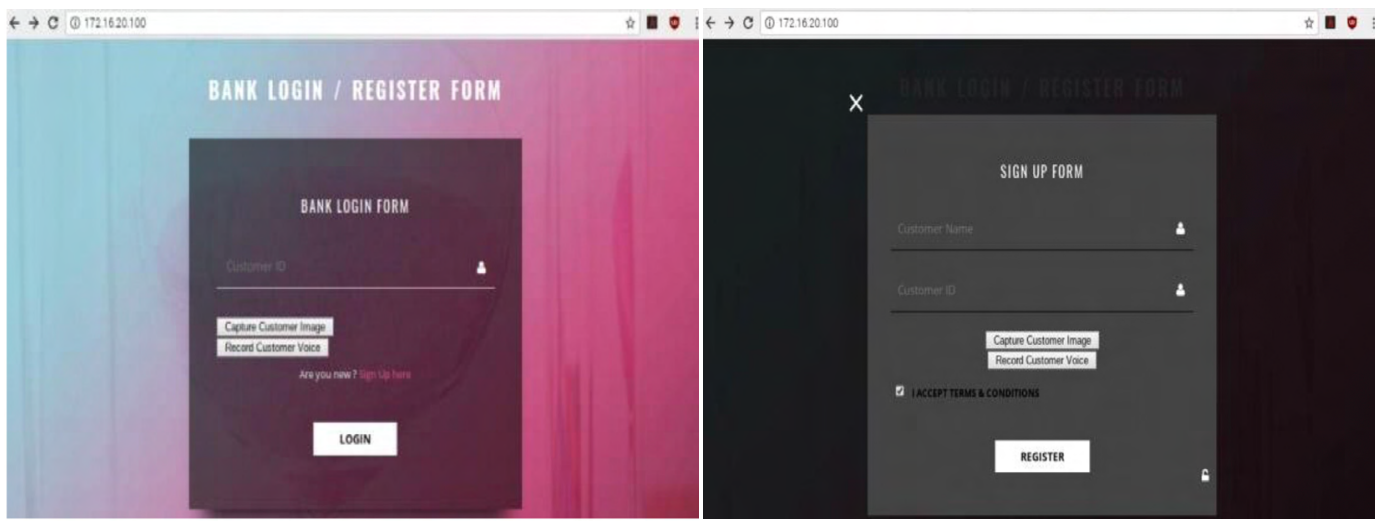

Figure 10. (a) Login form (b) Sign up form. 


\subsection{MATCHING}

The fresh images taken at the time of verification are compared in time by time manner with the stored templates for both voice and face image in our work.

Our work has been compared with others with methodology. Certain works have been designed for some intended purposes and they are designed to meet that. and the comparison of some works is given in Table 1.

Table 1. Comparison of previous works.

\begin{tabular}{|c|c|c|c|c|}
\hline References & $\begin{array}{l}\text { Biometric } \\
\text { trait }\end{array}$ & $\begin{array}{l}\text { Additional } \\
\text { Hardware } \\
\text { Module used }\end{array}$ & Algorithm & Intended Actions \\
\hline $\begin{array}{l}\text { Sahani et al. } \\
\qquad(2015)\end{array}$ & Face & $\begin{array}{c}\text { GSM/GPRS } \\
\text { module (For } \\
\text { transferring } \\
\text { information to the } \\
\text { owner) }\end{array}$ & $\begin{array}{l}\text { Eigen } \\
\text { Methodology }\end{array}$ & $\begin{array}{l}\text { The photograph of person enter into house is } \\
\text { captured and sent to the owner for allow/deny }\end{array}$ \\
\hline Baby et al. (2017) & Voice & Rasperry PI & $\begin{array}{l}\text { Voice to text } \\
\text { and database } \\
\text { storage }\end{array}$ & $\begin{array}{l}\text { To close the home, switch off the lights and } \\
\text { fans. }\end{array}$ \\
\hline Kaur et al. (2016) & Voice & Wiki, iCloud id & Voice to text & $\begin{array}{l}\text { It searches the missed iPhone, Helps to search } \\
\text { the movie, helps to search Wikipedia,reading } \\
\text { news, describe weather. }\end{array}$ \\
\hline $\begin{array}{l}\text { Gyulyustan \& } \\
\text { Svetoslav (2017) }\end{array}$ & Voice & Rasperry PI & $\begin{array}{l}\text { Hidden Markov } \\
\text { Model }\end{array}$ & $\begin{array}{l}\text { Speech recognition with intended words and } \\
\text { carry out the action behind that. }\end{array}$ \\
\hline $\begin{array}{l}\text { Senthilkumar } \\
\qquad(2014)\end{array}$ & Face & EICSRS platform & $\begin{array}{l}\text { Eigen faces } \\
\text { methodology }\end{array}$ & $\begin{array}{l}\text { If the user is not in the stored template, reject } \\
\text { the user. }\end{array}$ \\
\hline $\begin{array}{l}\text { Kishore Bhanse \& } \\
\text { Jaybhaye (2018) }\end{array}$ & Front image & Google API & $\begin{array}{l}\text { Machine } \\
\text { Learning, Neural } \\
\text { network }\end{array}$ & $\begin{array}{l}\text { To alert the user regarding correct user, or } \\
\text { intruder. }\end{array}$ \\
\hline Proposed & $\begin{array}{l}\text { Image and } \\
\text { voice }\end{array}$ & Rasperry PI & $\begin{array}{l}\text { Eigen } \\
\text { Methodology, } \\
\text { AES (image } \\
\text { Encryption) }\end{array}$ & $\begin{array}{l}\text { Both image and voice database information's } \\
\text { are stored in the encrypted format to avoid the } \\
\text { hackers template hacking. }\end{array}$ \\
\hline
\end{tabular}

\section{CONCLUSION}

This work proposes the design and the development of an interactive smart bank locker security system with the raspberry pi as the processor. The PC used for interaction can be replaced with low-cost processors which would provide the administrator with parameters of the entire remote device. This setup can be implemented in banking sectors for improved security of bank lockers. It can be used to avoid access of unauthorized persons. It can be 
easily used to track the intruders. Since, face and voice both the important features are used as the key factors, it provides an as an excellent security system. It reduces the risk of threat. Since encryption algorithms are employed, the customer images can be stored securely.

As a future scope, a separate application can be created to send the picture of the unauthorized customer through E-mail or through any other active social media in which the customers will be active and alert them with this intruder information. Also, the voice of the customer to be stored can be encrypted and then can be stored in the database. This will be an additional factor to enhance the security of bank lockers. 


\section{REFERENCES}

Baby, C. J., Munshi, N., Malik, A., Dogra, K. \& Rajesh, R. (2017). Home automation using web application and speech recognition. In 2017 International conference on Microelectronic Devices, Circuits and Systems (ICMDCS), Vellore, India. IEEE. https://doi. org/10.1109/ICMDC.S.2017.8211543

Gyulyustan, H., \& Svetoslav, E. (2017). Experimental speech recognition system based on Raspberry Pi 3. IOSR Journal of Computer Engineering (IOSR-FCE), 19(3), 107-112. https://doi.org/10.9790/0661-190302107112

Kaur, S., Sharma, S., Jain, U., \& Raj, A. (2016). Voice Command System Using RaspberryPi. Advanced Computational Intelligence An International fournal (ACII), 3(3), 4349.

Kishore Bhanse, V., \& Jaybhaye, M.D. (2018). Face Detection and Tracking Using Image Processing on Raspberry Pi. International Conference on Inventive Research in Computing Applications (ICIRCA). Coimbatore, India. IEEE. https://doi.org/10.1109/ ICIRCA.2018.8597246

Ramani, R., Selvaraju, S., Valarmathy, S., \& Niranjan, P. (2012). Bank Locker Security System based on RFID and GSM Technology. International fournal of Computer Applications, 57(18), 15-20. https://www.ijcaonline.org/archives/volume57/ number18/9213-3761

Sahani, M., Nanda, G., Sahu, A. K., \& Pattnik, B. (2015). Web-Based Online Embedded Door Access Control and Home Security System Based on Face Recognition. In 2015 International Conference on Circuit, Power and Computing Technologies (ICCPCT). Nagercoil, India. IEEE. https://doi.org/10.1109/ICGPCT.2015.7159473

Senthilkumar, G., Gopalakrishnan, K., \& Sathish Kumar, V. (2014). Embedded Image Capturing System Using Raspberry Pi System. International fournal of Emerging Trends \& Technology in Computer Science (IJETTCS), 3(2), 213-215. https://pdfs. semanticscholar.org/d2bf/70f60dff35086fd57b28525d7e5e6ea2eld0.pdf 
Shah, M., Patel, J., \& Patel, V. (2018). Development of Interactive Data Storage Unit Using Raspberry Pi. In 2018 International Conference on Inventive Research in Computing Applications (ICIRCA), pp. 825-830. Coimbatore, India. IEEE. https:// doi.org/10.1109/ ICIRCA.2018.8597217

Stallings, W. (2005). Cryptography and Network Security (4th ed.). Prentice-Hall, Inc.

Tabora, V. (2011). Face Detection Using OpenCV With Haar Cascade Classifiers. https:// becominghuman.ai/face-detection-using-opencv-with-haar-cascade-classifiers$941 \mathrm{dbb} 25177$ 


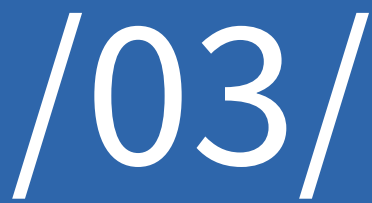




\section{SECURED TRANSMISSION IN DOUBLE CLUSTERED HETEROGENEOUS MOBILE WIRELESS SENSOR NETWORK}

T. Preethiya

Research Scholar. Department of ECE. Kalasalingam Academy of Research and Education, Srivilliputtur, (India). E-mail: preethiya.t@gmail.com ORCID: https://orcid.org/0000-0003-3504-1884

A. Muthukumar

Associate Professor. Department of ECE. Kalasalingam Academy of Research and Education,

Srivilliputtur, (India). E-mail: muthuece.eng@gmail.com ORCID: https://orcid.org/0000-0001-8070-3475

S. Durairaj

Principal. Dhanalakshmi Srinivasan Engineering College.

Perambalur, (India).

E-mail: rajsdr@rediffmail.com ORCID: https://orcid.org/0000-0002-7104-687X

Recepción: 05/12/2019 Aceptación: 13/01/2020 Publicación: 23/03/2020

\section{Citación sugerida:}

Preethiya, T., Muthukumar, A., y Durairaj, S. (2020). Secured Transmission in Double Clustered Heterogeneous Mobile Wireless Sensor Network. 3C Tecnología. Glosas de innovación aplicadas a la pyme. Edición Especial, Marzo 2020, 51-67. http://doi.org/10.17993/3ctecno.2020.specialissue4.51-67

\section{Suggested citation:}

Preethiya, T., Muthukumar, A., \& Durairaj, S. (2020). Secured Transmission in Double Clustered Heterogeneous Mobile Wireless Sensor Network. 3C Tecnología. Glosas de innovación aplicadas a la pyme. Edición Especial, Marzo 2020, 51-67. http://doi.org/10.17993/3ctecno.2020.specialissue4.51-67 


\section{ABSTRACT}

In recent years, Mobile Wireless Sensor Network (MWSN) has derived the attention of vendors and researchers as it being the state-of-art technology in the areas of battle field surveillance, medical and military application etc. The Mobile Double Cluster Head-Particle Swarm Optimization (MDCH-PSO) algorithm is proposed for optimization in hybrid mobile network with a heterogeneity. This paper proposes an algorithm Secure-MDCH (S-MDCH) to improve the security aspects of MDCH-PSO algorithm. In S-MDCH, intercluster and intra-cluster key generation algorithms are explained to prevent the network from malicious node attack and $\mathrm{CH}$ compromising. This ensures secure communication in the network. A unique mobile key " is used by all nodes to avoid malicious node from entering the cluster through handoff and to prevent 'information learning'. Simulation results shows that packet delivery ratio of the proposed algorithm is $8.25 \%$ higher than LEACH-M and average residual energy is improved by $2.802 \%$.

\section{KEYWORDS}

MWSN, Mobility, Heterogeneous, Security, Inter-cluster, Intra-cluster keys. 


\section{INTRODUCTION}

MWSN is a collection of an infrastructure less, self-organizing nodes with sensors to detect event occurrence that are connected wirelessly to form an arbitrary topology. The basic need of a network is to ensure a reliable data transmission, higher connectivity, lower energy consumption and increased life time. Many existing WSN application such as habitat monitoring, surveillance and medical application adopts mobility in its execution. Though, mobility makes the network complex its need make it as an advantage. Many research has revealed that mobility characteristics improves the overall network and QoS performance of the network.

\subsection{MWSN ARCHITECTURE}

As shown in Figure 1, in every sensor node a sensing unit, processing unit, transmission unit and a power unit are mandatory for its operation. The blocks mobilizer and position finding system are optional which can be activated based on the application. Enabling these optional blocks has provided a new paradigm to the sensor network 'Mobile Wireless Sensor Network' that can be used in many application creating a base for IoT and pervasive computing. The sensing unit comprises of sensor and analog-digital conversion circuit. The sensor can be selected from the wide range based on the application. The processing unit process the incoming data and stores it in a register. The transmitter is a communication model which provides radio transmission in the ground surface. The two components motor and chassis of mobilizer enables the node movement. These components are selected depending on the application.

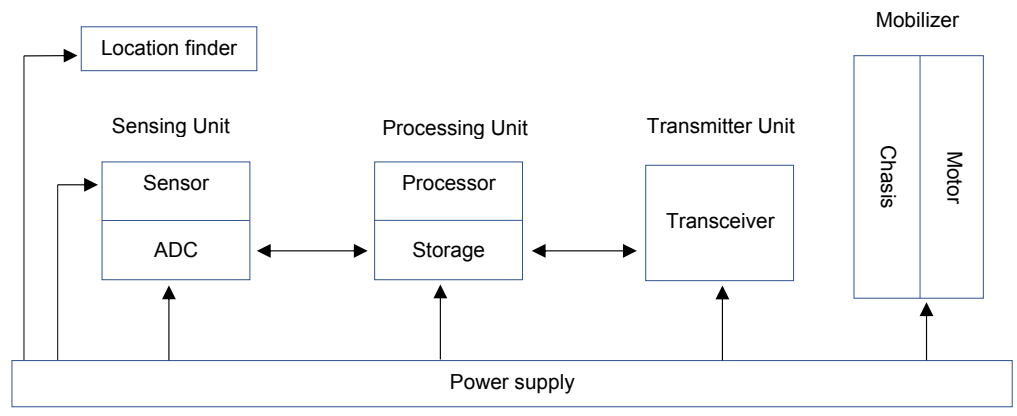

Figure 1. Architecture of MWSN. 
The MWSN node move over air, water or ground as application demands. Similarly, the chassis used also differs. The wheels, the caterpillars and the walking legs are the major options for movement of nodes in ground. To transform the electrical energy into the mechanical energy required for wheel or caterpillar rotation or leg movement, the mobile node use the motors. Each node uses two motors for its direction change. According to Gungor and Hancke (2009), Srivastava (2010) and Rathee, Singh and Nandini (2016), the challenges of MWSNs are identified as processing speed, network heterogeneity, scalability, hardware cost, deployment, Size of memory and battery, balanced traffic, dynamic topology, mobility, coverage, energy consumption, localization, node failure, QoS, fault tolerance, wireless connectivity, and security. The addition of security features to the MWSN's make it more compatible.

Messai (2014) and Singh, Singh and Singh (2016) has identified the possible security threats in mobile networks as:

\section{Malicious node attack-An intruder can act as a handoffnode and falsify the local data}

Being a mobile network there will be a frequent topology change and it is handled by handoff. In this case both hard and soft handoff takes place. In such case, a malicious node can act as a handoff node from adjacent cluster and transmit false event data to the female node thereby wasting the resources.

\section{Learning information table}

A malicious node can learn entire cluster details through female node communication that may violate message confidentiality and authentication.

\section{Compromised CH attacks}

The $\mathrm{CH}$ node is compromised by the attacker which creates black hole attack, selective forwarding attacks in the network.

This paper proposes an intra-cluster and inter-cluster key generation algorithm for double cluster head heterogeneous mobile hybrid network. In general, mobility is the movement of node from one place to the other. Security is an important aspect in any mobile network 
as it changes its topology dynamically. This allows the intruders to spoof the transmitted information and create other attacks in the network. For a DCH network, a malicious node can easily enter and modify the information table. This is avoided by a unique mobile key by the mobile node.

\section{LITERATURE SURVEY}

Xuegong and Chen (2010) have introduced a 'Double Cluster-Head topology Control Algorithm' (DCCCA) for a heterogeneous network. MCH is selected based on weight. Here, Main $\mathrm{CH}(\mathrm{MCH})$ collects the data and transmitted by an Assistant $\mathrm{CH}(\mathrm{ACH})$ to the next CH. A chain based algorithm 'Power-Efficient Gathering in Sensor Information Systems with Double Cluster Head (PDCH)" is proposed by Linping, Wu, Zhen and Zufeng (2010), where the cluster heads are classified as MCH and secondary $\mathrm{CH}$. The parameters such as energy and distance to $\mathrm{CH}$ were used for $\mathrm{CH}$ selection. A node with higher tag value and with more than two neighbors is elected as $\mathrm{MCH}$ and any one of its neighbors in the next level is elected as secondary CH. Because of this, node that is far away from CH node takes too much energy to send its own data to cluster head from network.

Xiao and Deng (2010) recommended a 'Double Head Static Cluster' (DHSG) algorithm where the problems related to uneven distribution of nodes are addressed. The MCH is selected in thick and $\mathrm{ACH}$ in thin area and they are used to reduce single cluster head's energy consumption. An algorithm called 'Multiple Cluster-heads Routing Protocol' (MCHRP) is proposed by Da, Liu, Jiao and Yue (2011). This MCHRP algorithm uses maxmin approach for the election of $\mathrm{CH}$. The $\mathrm{MCH}$ selection is based on residual energy and frequency of being $\mathrm{CH}$ and Vice $\mathrm{CH}(\mathrm{VCH})$ election is based on residual energy, distance between node to $\mathrm{CH}$, distance between node to base station and frequency of being $\mathrm{CH}$.

Suresh and Selvakumar (2014) have proposed the SKADC algorithm uses an inter-cluster and intra-cluster keys to provide security for static WSN. It uses SHA-1 MAC for node authorization. The digest size of SHA-1 MAC is 20 bytes and 80 steps to create a digest size. In real time, TinySec frame work will have 29 bytes of information to transmit the message. With SHA-1 MAC, the remaining 9 bytes are left blank which results in waste of resources. This algorithm is proposed for double cluster architecture. Four different keys 
are generated in intra-cluster key generation. A multiplicative element is obtained to avoid compromising of node.

In literature, numerous algorithms are proposed for double CH selection and energy efficiency. The above study explains double cluster head mechanism for wireless sensor network. The existing algorithms do not focus the security aspect of the network. A mobile network with double cluster head has to face the more security issues than the single clustered architecture. This paper explains about the security aware energy efficient double cluster head algorithm for a mobile network.

The contribution of this paper given below.

1. It is proposed for double clustered heterogeneous hybrid mobile network.

2. It uses SHA-224 algorithm for MAC generation. The number of keys generated in intra cluster communication is reduced.

3. A unique individual key is given to all node by $\mathrm{F}$ nodes for transmitting.

4. A mobility key is also generated to learn mobility in the network.

5. A unique multiplicative element is obtained periodically to prevent attacker from knowing keys.

\section{PROPOSED WORK}

\subsection{S-MDCH}

There are four phases in the Secure-MDCH (S-MDCH) algorithm as shown in Figure 2. In this algorithm, there are two $\mathrm{CH}$ namely male $\mathrm{CH}$ (Temporary $\mathrm{CH}$ ) that is elected among the member node and female node is heterogeneous immobile node that acts as the backbone of the network. 


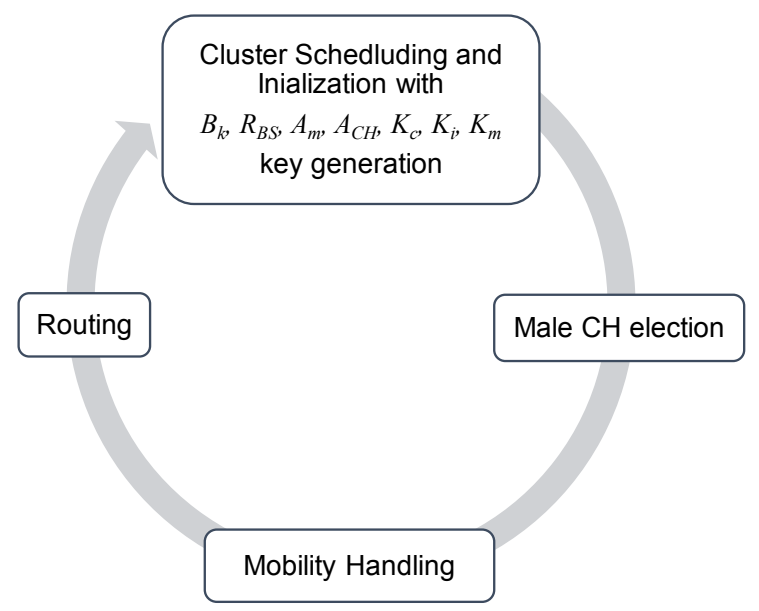

Figure 2. Phases of S-MDCH algorithm.

\section{a) Cluster scheduling algorithm and initialization phase}

In this phase, initial clustering is done (i.e.) member nodes are registered or scheduled with the female node using the received signal strength of every node to get connected with female node. The HELLO_PKTS are generated and flooded by the female sensor node. It consists of the source address of the female sensor node and information fields. Similarly, the REPLY_PKTS from each node contains source and destination address field, information field. Each node sends its node id, residual energy, one hop neighbors, and distance to female node in its information field and time stamp in its REPLY_PKTS. The possible security issue in this phase is 'learning information table'. A malicious node that acts as member node may register with female node and acquire its cluster details.

The female node floods a HELLO_PKT to its member node which in turn sends a REPLY_PKT which has its digital signature in addition to the data. The female node uses a verifying algorithm to the data received and if the result is true, data from that member node is accepted and store in its table.

b) PSO based male CH Selection phase

The temporary male selection is done using Particle Swarm Optimization. The fitness value is calculated as follows: 


$$
\begin{gathered}
\text { fitness }(i)=w_{1} E_{\text {residual }}(i)+w_{2} \text { Distance }_{\text {tofemale }}+w_{3} \text { Node density }(i) \\
E_{\text {residual }}=E_{\text {initial }}-\left(E_{T x}+E_{R x}+E_{\text {mobile }}\right) \\
\text { Distance } \text { tofemale }=T x_{\text {range }} \times M_{s} \\
\text { Node density }=\frac{\text { Node degree }_{\text {dee }}}{\pi \times \varphi}
\end{gathered}
$$

where $w_{1}, w_{2}$ and $w_{3}$ are constants between 0 and 1 .

\section{c) Mobility handling Phase:}

The female node updates its "information table" after every transmission by HELLOREPLY packets. In short, if a node does not reply, the female node consider it as an 'away node' and remove its data from the table after waiting till its next HELLO-REPLY packets. Meanwhile, if a new node enters the cluster, female node obtain it's $K_{m}$ and decrypts that to authenticate that node. Finally, female node consider it as the 'recent node' and update its information in the table through the subsequent HELLO-REPLY message.

\section{d) Routing Phase:}

The female node directly gathers the information from its member node that are registered in the table. The routing phase involves the intra and inter-cluster key generations for secure transmission of data. The female node gathers the event occurrence from all the nodes and aggregate it $D_{\text {agg }}$. This is forwarded to male $\mathrm{CH}$ to reach the base station with public and private keys. The algorithm is described in next sub section.

e) SHA-224 algorithm:

The data field is 29 bytes for a TinySec authentication frame work. So, a message digest used for authentication should be 29 bytes. The digest sizes of SHA-1, SHA-224, SHA256, SHA-384, and SHA-512 are 20, 28, 32, 48, 64 bytes respectively. The SHA-256, SHA-384 and SHA-512 are excluded since their digest size exceeds the limit. So, SHA-1 and SHA-224 are the choices. In an event sensing mobile environment the computation time should be less. Nunoo-Mensah, Boateng and Gadze (2015; 2017) has clearly proved that SHA-224 has less execution time when compared with SHA-1. So, we have adopted SHA-224 algorithm in S-MDCH for MAC generation. 


\subsection{INTER CLUSTER KEY GENERATION}

1. Base station broadcasts initial $B_{k}$ to Female ' $F$ '

$$
\begin{aligned}
& B_{k}:\left\{I M_{i}\left\|S N_{i}\right\| L_{p}\right\} \\
& \text { if } H\left(S N_{i}\right)=S N_{i-1}
\end{aligned}
$$

then

$$
\begin{aligned}
& B_{k} \text { is autorized } \\
& \text { else } \\
& F_{i} \text { drops } B_{k} \\
& \text { endif }
\end{aligned}
$$

2. Update $\mathrm{L}_{\mathrm{P}}$ to reach base station

3. After recievig $B_{k}, F_{i}$ sends reply $R_{B S}$ to base station

$$
R_{B S}=\left\{E\left(I D\left(F_{i}\right), K_{i}, K_{p r i}\left(F_{i}\right) \| E\left(M A C\left(R_{e} \| I D\left(F_{i}\right), K_{i}, K_{p r i}\left(F_{i}\right)\right)\right)\right)\right\}
$$

4. BS after recieving $R_{B S}$ from $\mathrm{CH}$ validates the message and generates an authorization message $\left(A_{m}\right)$ to every $\mathrm{CHs}$.

5. BS after $K_{r}$ from $R_{B S}$ and adds it to $A_{m}$ and univasts to Female nodes.

$$
A_{m}=\left\{I D\left(F_{i}\right), K_{i}, K_{p r i}\left(F_{i}\right) \| E\left(M A C\left(R_{e} \| I D\left(F_{i}\right), K_{i}, K_{p r i}\left(F_{i}\right)\right)\right)\right\}
$$

6. CH upon recieving the $A_{m}$ verifies and decrypts it and generates level key $(K L i)$ for its child cluster heads. Then if forwards the cluster head authorization message $A_{C H}$ to child cluster heads.

$$
A_{C H}=\left\{I D\left(F_{i}\right) \| E\left(K L i, K_{r}\right)\right\}
$$




\subsection{INTRA-CLUSTER KEY GENERATION}

7. ' $\mathrm{F}^{\mathrm{c}}$ broad casts routing message $R_{\text {req }}$ to its $m_{i}$.

8. Each mi broadcasts the $R_{\text {req }}$ message to its neighbors and obtains the hop distance to reach ' $\mathrm{F}$ '.

9. Nodes with less number of hops to reach $\mathrm{F}$ will act as Male node ' $\mathrm{M}$ ' ' and if twho nodes have same hop count a node with less mobile speed is eleccted as ' $\mathrm{M}$ '.

10. $m_{i}$ broadcasts $R_{\text {req }}$ message in the reverse path trasversed by request message.

11. The following kets are generated for secure communication within the cluster.

a. Cluster key $K_{c}$ - to be shared with entire cluster.

b. Individual key $K_{i}$ - to be shared with F.

c. Mobility key $K_{m}$ - generated when a new node joins the cluster due to mobility.

12. To prevent attacker from confronting the keys, it is generated by a source multiplicative element ' $\mathrm{z}$ ' with a random key values.

a. Note: These keys changes with when ' $\mathrm{z}$ ' changes.

13. F can request $\mathrm{BS}$ for new ' $\mathrm{z}^{6}$ periodically which avoids node compromising.

\section{RESULTS AND DISCUSSION}

Simulation area is assumed to be $600 \mathrm{~m} \times 600 \mathrm{~m}$ with 50 nodes distributed randomly. Sink node is placed at $(300,300)$ to gather the occurrence of events from various locations. The mobility model used is Random Way Point model. This is chosen because it is a model that can use pause time between changes and speed. The simulation results are recorded at mobility speed $20 \mathrm{~m} / \mathrm{sec}$ to study the performance of network. The pause time is set to $50 \mathrm{sec}$. The initial energy of member node is set to 2 Joules and a female node is 10 Joules. Table 1 shows the simulation parameters considered for the energy model of the network and simulated using network simulator 2.35 . 
Table 1. Simulation parameters.

\begin{tabular}{|c|c|}
\hline Parameter & Value \\
\hline Deployment & Random \\
\hline Energy Consumption per bit & $50 \mathrm{~nJ} / \mathrm{bit}$ \\
\hline$\varepsilon f s$ & $10 \mathrm{pJ} / \mathrm{bit} / \mathrm{m} 2$ \\
\hline$\varepsilon m p$ & $0.0013 \mathrm{pJ} / \mathrm{bit} / \mathrm{m} 4$ \\
\hline Data packet size & $512 \mathrm{bytes}$ \\
\hline C1 and C2 & 2 \\
\hline W & 0.9 \\
\hline
\end{tabular}

A malicious node has been introduced to study the performance of the network. Figure 3 shows the packet delivery ratio against the offered load. The delivery ratio gets dropped as the offered load increases in the network in both algorithms. The delivery ratio is $8.25 \%$ higher than the LEACH-M algorithm. The obtained PDR is consistent during less offered load because of the high pause time which avoids topology change for $50 \mathrm{sec}$. This will prevent any malicious node from entering the cluster due to handoff. Also, the entire transmission takes place in a stable energy efficient secured path. The data transmission takes place using a unique private key and hash value for each transmission. Further increase in load, will create congestion in the network thus PDR decreased. This can be improved by varying the mobile speed of each node.

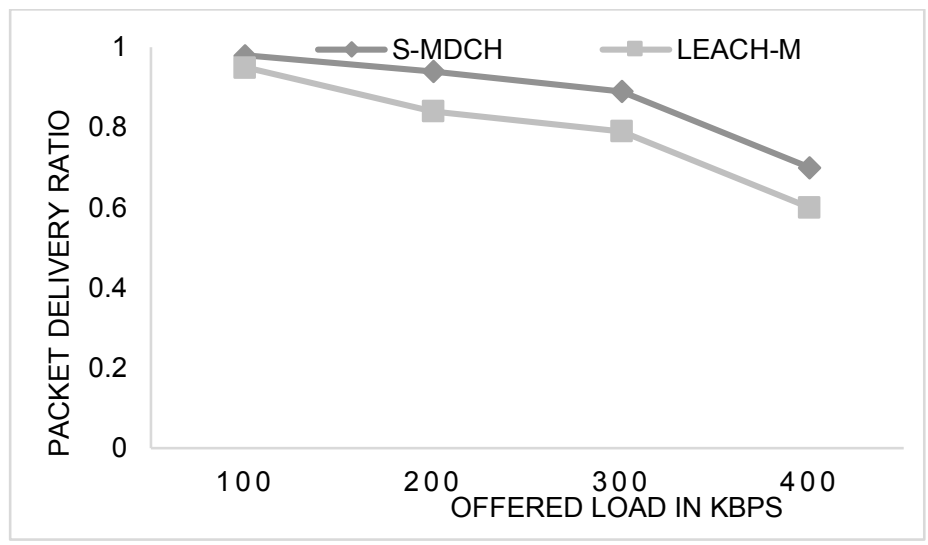

Figure 3. PDR versus Offered load.

Generally in mobile network, mobility is a major reason that contributes to packet drop. If the route to destination is not available then the packets drop at the source node and if the next hop is not available then packet loss occurs at intermediate nodes. Also, malicious 
node may cause more packet dropping which forwards only the selective packets to the destination and drops the other. This affects the message integrity at the base station. In the proposed algorithm, the malicious is node is identified in the registration phase. Even if malicious node receives the packet it is not able to modify the data packet. From the Figure 4 , it seen that packet loss is $8 \%$ higher in LEACH-M when compared to the S-MDCH. This is because LEACH-M has not been designed to provide security rather it simply carry forward the packet. As the offered load increases, the packet loss increase in both algorithms, because higher load with more mobile nodes causes congestion and frequent change in path to the destination. However, this is reduced by the pause time of the nodes and some nodes still may cause it to happen. (i.e.) a node in the path has completed its 50 sec pause time during transmission. The other way of improving packet loss is by adhering varying mobile speed for each node.

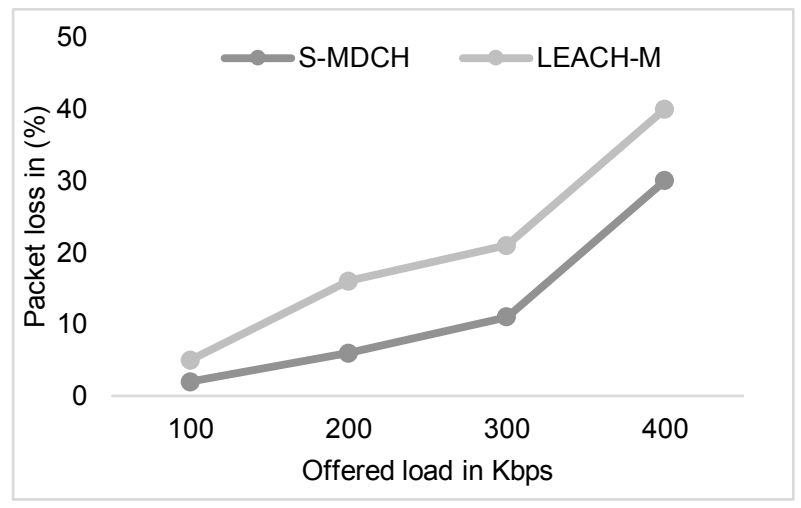

Figure 4. Packets dropped versus Offered load.

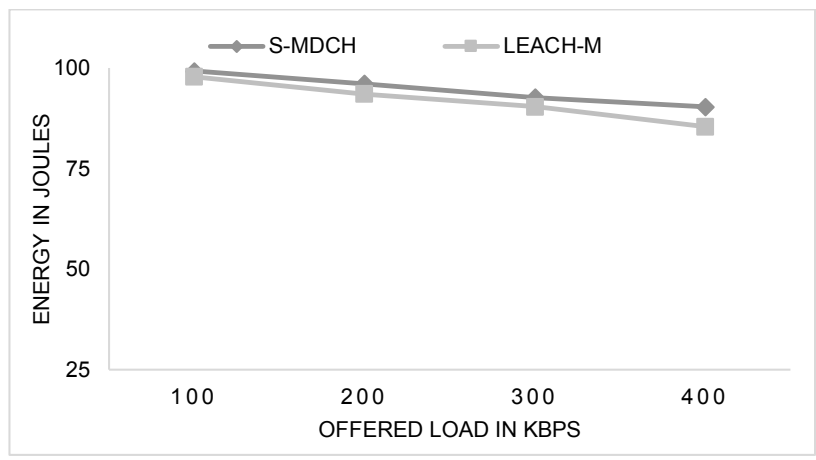

Figure 5. Average residual energy versus Offered load. 
When compared to static node, mobile node spends one part of its energy in mobility of node. This is may vary due to additional components attached to mobilizer unit. Figure 5 represents the average residual energy of member nodes (initial energy is 2J) in the network. The total number of nodes is $50 * 2 \mathrm{~J}=100 \mathrm{~J}$. As shown in Figure 5, the average residual energy of the network in S-MDCH is $2.802 \%$ higher than the LEACH-M algorithm. In S-MDCH algorithm, a time-stamping based handoff mechanism is used whereas in LEACH-M, a simple handoff mechanism is used. The reduced number of key generation reduces the overhead transmission which results in effective energy minimization.

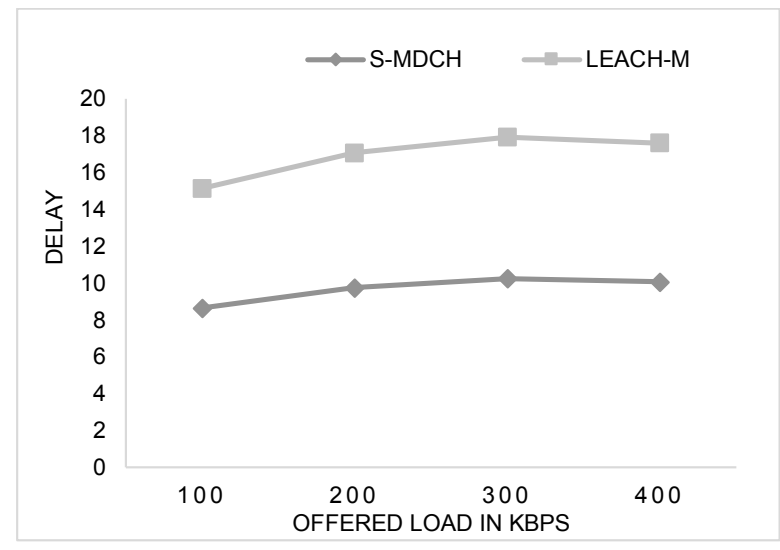

Figure 6. Delay versus Offered load.

Figure 6 shows the Offered load Vs delay. From the Figure 6, it is analyzed that average delay of S-MDCH is 7.25 times less than LEACH-M algorithm. The reason behind this is, in LEACH-M algorithm the CH monitors the member nodes and generates all inter-cluster and intra-cluster keys for secured communication. In the proposed S-MDCH algorithm inter-cluster keys are generated by the female node and intra-cluster keys by the member nodes and female. The female node generates the cluster key and unicasts it to all member nodes. Similarly, if a node wants to transmit it uses its individual key rather than using neighbor keys. Therefore all nodes concentrate on communication rather monitoring.

\section{CONCLUSION}

This proposed S-MDCH algorithm improves the security aspects of MDCH-PSO algorithm. The proposed algorithm uses SHA-224 algorithm which reduces the execution 
time in a TinySec framework. The number of keys used in intra-cluster communication is reduced and a mobility key is introduced to authenticate the mobile node during handoff. Simulation results shows that packet delivery ratio of the proposed algorithm is $8.25 \%$ higher than LEACH-M, average residual energy is improved $2.802 \%$ and delay by 7.25 times less than LEACH-M algorithm. In future, algorithm can be adopted to the network with varying mobility speeds. 


\section{REFERENCES}

Da, T., Liu, X., Jiao, Y., \& Yue, Q. (2011). A load balanced multiple Cluster-heads routing protocol for wireless sensor networks. IEEE 13th International Conference on Communication Technology, Finan, 656-660.

Gungor, V. G., \& Hancke, G. P. (2009). Industrial wireless sensor networks: Challenges, design principles, and technical approaches. IEEE Transactions on Industrial Electronics, 56(10), 4258- 4265. https://doi.org/10.1109/TIE.2009.2015754

Linping, W., Wu, B., Zhen, C., \& Zufeng, W. (2010). Improved algorithm of PEGASIS protocol introducing double cluster heads in wireless sensor network. In International Conference on Computer, Mechatronics, Control and Electronic Engineering, Changchun, pp. 148151. https://doi.org/10.1109/CMCE.2010.5609618

Messai, M. L. (2014). Classification of Attacks in Wireless Sensor Networks. International Congress on Telecommunication and Application' 14 University of A. MIRA, Bejaia, Algeria, pp. 23-24.

Nunoo-Mensah, H., Boateng, K. O., \& Gadze, J. D. (2015). Comparative analysis of energy usage of hash functions in secured wireless sensor networks, International Fournal of Computer Applications, 109(11), 20-23. https://doi.org/10.5120/19233-0968

Nunoo-Mensah, H., Boateng, K. O., \& Gadze, J. D. (2017). Tamper-aware authentication framework for wireless sensor networks. IET Wireless Sensor Systems, 7(3), 73-81.

Ramasamy, V. (2017). Mobile Wireless Sensor Networks: An Overview. Wireless Sensor Networks Insights and Innovations, Chapter 1. https://doi.org/10.5772/intechopen.70592

Rathee, A., Singh, R., \& Nandini, A. (2016). Wireless sensor network - challenges and possibilities. International Journal of Computer Applications, 140(2). https://www. ijcaonline.org/archives/volume140/number2/24563-2016909221

Singh, R., Singh, J., \& Singh, R. (2016). Attacks in wireless sensor networks: a survey. International Fournal of Computer Science and Mobile Computing, 5(5), 10-16. 
Srivastava, N. (2010). Challenges of next-generation wireless sensor networks and its impact on society. Fournal of Telecommunications, 1(1), 128-133.

Suresh, D., \& Selvakumar, K. (2014). Secure Key-Tree Architecture for Double Cluster Based Routing in Wireless Sensor Network. International Journal of Applied Engineering Research, 9(22), 16143-16157.

Xiao, Y., \& Deng, L. (2010). A double heads static cluster algorithm for wireless sensor networks. 2nd Conference on Environmental Science and Information Application Technology, Wuhan, pp. 635-638. https://doi.org/10.1109/ESIAT.2010.5568343

Xuegong, Q., \& Chen, Y. (2010). A control algorithm based on double clusterhead for heterogeneous wireless sensor network. 2nd International Conference on Industrial and Information Systems, Dalian, pp. 541-544. https://doi.org/10.1109/ INDUSIS.2010.5565790 


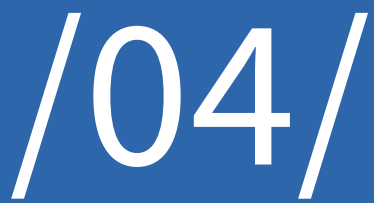




\section{BALKING AND RENEGING OF BATCHES IN VOD APPLICATIONS}

R. Vanalakshmi

Research Scholar, Department of Mathematics, Kalasalingam Academy of Research and Education.

Krishnankovil, (India).

E-mail: vanalakshmi31@gmail.com ORCID: https://orcid.org/0000-0002-3440-5044

S. Maragathasundari

Associate Professor, Department of Mathematics, Kalasalingam Academy of Research and Education.

Krishnankovil, (India).

E-mail: maragatham01@gmail.com ORCID: https://orcid.org/0000-0003-1210-6411

K. S. Dhanalakshmi

Assistant Professor, Department of Electronics and Communication Engineering,

Kalasalingam Academy of Research and Education.

Krishnankovil, (India).

E-mail: k.s.dhanalakshmi@klu.ac.in ORCID: https://orcid.org/0000-0001-6285-3656

Recepción: 05/12/2019 Aceptación: 08/01/2020 Publicación: 23/03/2020

\section{Citación sugerida:}

Vanalakshmi, R., Maragathasundari, S., y Dhanalakshmi, K. S. (2020). Balking and reneging of batches in vod applications. 3C Tecnología. Glosas de innovación aplicadas a la pyme. Edición Especial, Marzo 2020, 69-89. http://doi.org/10.17993/3ctecno.2020.specialissue4.69-89

\section{Suggested citation:}

Vanalakshmi, R., Maragathasundari, S., \& Dhanalakshmi, K. S. (2020). Balking and reneging of batches in vod applications. 3C Tecnología. Glosas de innovación aplicadas a la pyme. Edición Especial, Marzo 2020, 69-89. http://doi.org/10.17993/3ctecno.2020.specialissue4.69-89 


\section{ABSTRACT}

This paper researches an examination on Video-on-request (VOD) alludes to video benefits in which customers can demand any video program from a server whenever. VOD has significant applications in stimulation, training, data, and promoting, for example, film onrequest, remove learning, home shopping, and intelligent news, so on. Versatility in gushing limit can be cultivated by strategies for interest clumping, in which requests for a video touching base inside a time allotment are collected together (i.e., batched) and presented with a lone multicast stream. The target here is to achieve the trade between the multicasting cost and customer delay in the system. We analyze different clustering schemes (as far as customers concede experienced, the amount of customers assembled in each bunch, thus on...), and how framework benefit can be helped given client's reneging conduct. This issue of postponement in patch up procedure and separate happening in VOD writing computer programs is drawn nearer through lining hypothesis in this investigation. Lining disposition characterizes the mistake I the systems administration and gives out the expected plans to be passed out to limit the blunder happening assets. It likewise presents the idea of support period after the fruition of administration. Numerical delineation and an expand graphical investigation are completed toward the conclusion to approve the model. It gives a reasonable pondered the applied investigation of lining hypothesis in VOD systems.

\section{KEYWORDS}

Balking, Reneging, Batch arrival, Emergency vacation, Compulsory vacation. 


\section{INTRODUCTION}

VIDEO-ON-DEMAND (VOD) spilling administration over remote systems is exponentially expanding with creative advances in keen cell phones. To give the VOD spilling administration, high caliber of administration (QoS)requirements ought to be met, for example, high transfer speed necessity, low administration idleness, low administration blocking rate, and so forth. Also, clients need to access to any substance, whenever, anyplace, on any gadget. The conventional unicast transmission has focal points such straightforwardness, no administration inactivity (i.e., start-up postponement), and simple execution of client heterogeneities, for example, cushion limit and channel conditions. Be that as it may, unicast isn't versatile. The transfer speed utilization increments directly as the client solicitations increment. In this light, a few multicast/communicate based transmission plans are being concentrated to deal with the development of portable video traffic. They are versatile and productive as far as data transmission necessity. All things considered, the administration by and large produces extreme idleness and can't promptly think about client heterogeneities. Clumping, fixing, and occasional telecom plans are in the classes of the multicast/communicate based transmission.

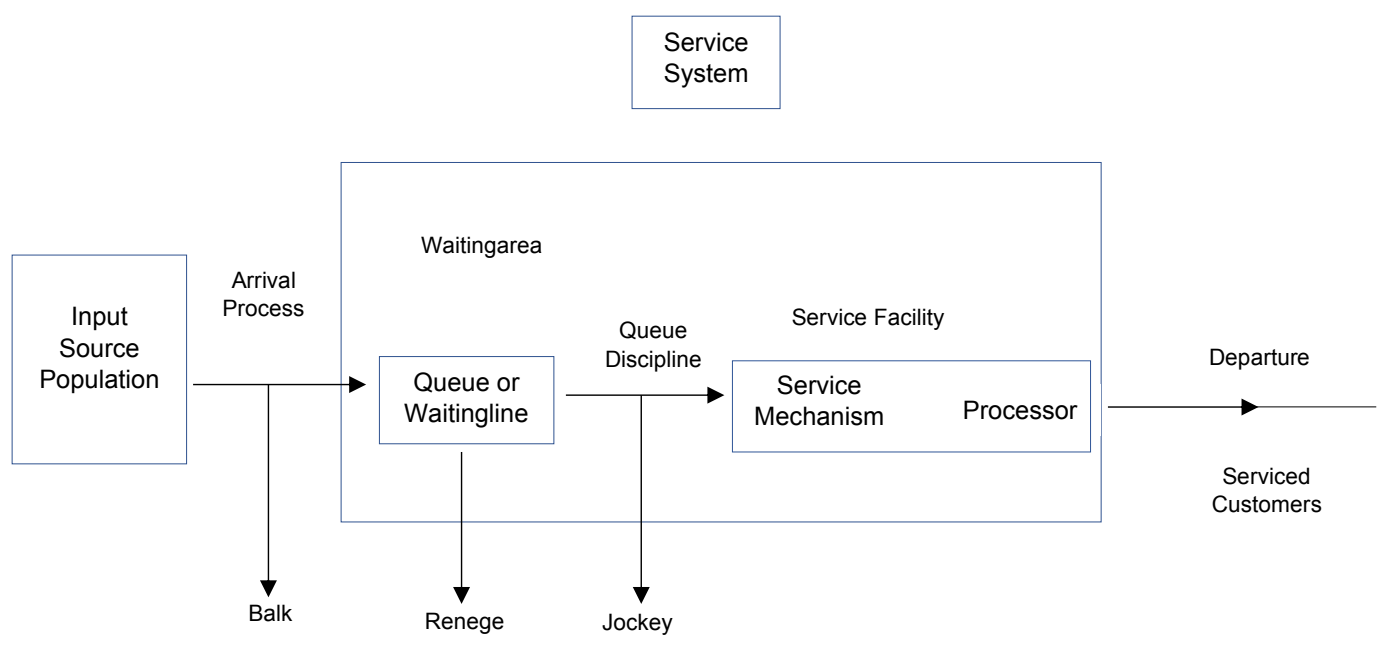

Among them, clumping can ensure the administration inactivity inside specific limits for both mainstream and disliked substance The record related qualities of a VOD application incorporate gushing data transfer capacity, size of the documents, number of video titles, and video fame. The spilling transfer speed of a video, b0, relies upon the video pressure 
plan utilized (e.g., MPEG-I, MPEG-II, movement JPEG, and so forth). It can go from under $1 \mathrm{Mbps}$ to more than $10 \mathrm{Mbps}$. Gushing data transfer capacity likewise relies upon the encoding technique utilized (e.g., Constant-piece rate, Variable-piece rate, etc.). The size of a video document is the genuine extra room the record expends in a capacity medium. It might run from $\sim 10 \mathrm{MB}$ (promoting cuts) to more than $\sim 1$ GB (motion pictures). All documents in a VOD application may not be of a similar size. In MOD (film on-request), for instance, the record size is probably going to be comparable or "homogeneous," with each document of about, state, an hour and a half playback time. Then again, record estimate in intelligent news condition can be fairly "heterogeneous," contingent upon the bit of news and whether it is a narrative or not. Somewhere close to the "limits" might be home shopping, in which record size may run from $\sim 5 \mathrm{MB}$ to $\sim 30 \mathrm{MB}$ (20 seconds to 2 minutes). applications focused to overall population are probably going to have a greater number of titles than applications for a littler gathering of clients. Various recordings have diverse access recurrence. The ubiquity of a video is characterized as the likelihood for the video to be gotten to or picked by any approaching solicitation.

Singh (2016) showed that the gushing design and security issues were the difficulties looked by VOD. Jain and Bhargava (2008) worried about the examination of questionable server mass entry retrial line with two class non-preemptive need endorsers. Juhn, and Tseng (1998) presents another telecom plot, which can bolster live recordings and decrease the holding up time to 8 minutes. McManus and Ross (1996) presented a particular transport and transmissions conspire for video - on-request (VOD) called steady - rate transmission and transport (CRTT). Maragathasundari (2015) derived the execution measures for a mass section queuing model of three periods of organization with different journey strategies. Maragathasundari, Anandapriya, Gothaiammal and Gowri (2017) described a non-markovianqueuing model in which entry was taken after a Poisson method. Maragathasundari and Karthikeyan (2016) investigated a mass queuing model with short and long escape. Maragathasundari, Srinivasan and Ranjitham (2014) examined a bunch landing queuing arrangement of stage get-away with two phases ofadministration dependent on a Bernoulli plan. Alomari and Sumari (2011) gave measurable data about the web, correspondences and cell phones and so forth. Abeywickrama and Wong (2013) featured that vital advancement of a nearby capacity inside the system empowered the 
administrations to be conveyed with improved nature of administration to the client. Kanrar (2012) displayed an effective estimation of the transfer speed necessity for the distinctive design. Gupta (2013) should attempt to respond to addresses identified with innovative and administrative difficulties looked by IPTV and the requirements identified with its effective usage in India and broke down the capability of IPTV as an apparatus of instruction in setting to the changing worldview of educating - learning strategy - and Pedagogy. Viswanathan and Imielinski (1996) gave expository and trial assessments of pyramid broadcasting dependent on its execution on an Ethernet LAN. Van Den Broeck, Pierson and Lievens (2007) orchestrated learning on existing review rehearses just as the Video-onrequests new affordances. He and Liu (2009) demonstrated that VOVO was adaptable and compelling, giving short start up latencies and great execution in VCR intuitive.

\subsection{PROCESS CARRIED OUT IN VOD PROGRAMMING}

Different applications have different performance requirements. We list here six such requirements:

\section{Batch arrival:}

In VOD, every client is appointed its own committed uncast stream. Henceforth clients appreciate fantastic adaptability in interacting with the server while viewing their videos.

\section{First Stage Service: Multicast delivery VOD service}

In a multicast conveyance VOD administration, motion pictures are made accessible just toward the start of spaces. The opening term is on the request of minutes (in our investigation we utilize the range from 30 seconds to 20 minutes). Clients making a solicitation will in this way need to pause, by and large, a large portion of a space span before the motion picture can begin. For short opening lengths (state 6 minutes) this ought not influence "onrequest" nature of the framework. At the point when the server gets a client demand it decides whether assets are accessible to support the solicitation. The server utilizes data about exceptional solicitations and the accessibility of assets to accept or reject demands. Note that the server performs clear "First Come, First Serve"scheduling. Solicitations are not appointed need, and no solicitation is denied if assets exist to service it. Clients are educated through reaction messages whether their solicitation is acknowledged or denied. 
All demands that touch base during the present space are booked or dismissed before the finish of the opening.

Emergency vacation during the time of service:

The Long starts up deferrals are considered as Emergency Vacation during the season of administration. While a long start-up postpone is bothersome, for the most part clients are eager to hold up longer under the accompanying conditions:

a. Delay guarantee: Clients might be all the more eager to pause on the off chance that they are certain that they can watch their recordings at a specific time, regardless of whether the time is potentially minutes (or even hours) after the fact. This is the rule behind postpone ensure frameworks, such as deterministic deferral (in which clients experience comparable deferral) or reservation system, in which clients save recordings to be shown at a specific later time

b. User interactions: We list two such requirements here: response time of the interactions and control granularity of the interactions. (i) Response time of the interactions (ii) Control granularity of user's interactions

c. Others: A VOD framework should offer adequate video quality. Various administrations may require distinctive video quality relying upon the class of the clients, application, and so on. Besides, the planning arrangements utilized in a server ought to be reasonable. For instance, in film on-request, a client who happens to demand a disagreeable motion picture ought not to be separated for a client mentioning an increasingly famous motion picture, if the two are charged the equivalent.

\section{Second Stage Service:}

Interactive VOD Service: In intelligent VOD services, a client viewing a motion picture will be able to control the playout of the motion picture. Client association with on-request films can be like the interactivity customers have when they lease a motion picture and watch it utilizing a video tape machine. In addition, the utilization of advanced video will empower new ideal models for intelligence. The varieties of the conventional VGR elements of delay, rewind, and quick forward. 


\section{Service Interruption:}

Delay is occurring between separate and fix process - start-up deferral:

We characterize "start-up deferral," as the holding up time from the minute when a client at first presents a video demand until the minute when the client starts to see the video. It is along these lines the absolute holding up time before the mentioned video is gushed. Clearly, start-up deferral is an irregular variable whose esteem relies upon where the client is in the line, what the client's need class is, or even the video mentioned. We recognize here start-up deferral from the reaction time of client connections. While start-up deferral is the sitting tight time for a client before the mentioned video is shown, the reaction time of client collaborations is the inertness from the season of issuing a control order to the genuine scene change in an on-going video session. In this manner, start-up postponement can be longer than the reaction time of client communications. Diverse VOD applications have distinctive least start-up postpone necessities. The necessity may rely upon to what extent a client sees the video, i.e., the solicitation's holding time.

\section{Balking during Repair:}

If the VOD server isn't reachable and for upkeep reason, the Balking of customers may occur.

\section{Reneging during delay:}

Depending on the holding up time achieved, a customer may drop its interest and leave the system (i.e., renege). The reneging behavior of the customers is a basic idea in the structure of a nearby VOD structure and the essential interest clumping plans.

\section{Compulsory Vacation after completion of the service:}

Video servers: The video servers store various motion pictures (described by their length, fame and gushing information rate) open by the clients. Every server has limited stockpiling and spilling capacities. Such assets are viewed as constantly accessible and one might say effectively paid for the accessible gushing limit might be parceled or shared among the motion pictures. In a close VOD framework, the fundamental issue is to properly allot the constrained gushing ability to the different demands by methods for bunching. 
Necessary excursion must be taken by the video server subsequent to finishing the gushing administration. To conquer the solid issue of clog in VOD programming a lining model is proposed in the present work. The lining instrument is created dependent on the likelihood circulation in various scope of correspondence. A procedure did in VOD application is totally changed into a lining issue. As clarified above in VOD application, the procedure comprises of rendering various phases of administration, a crisis excursion (upkeep work or set up time work) before the second phase of administration. After the culmination of the second phase of administration, mandatory excursion (Compulsory support work) is done. Next, because of blockage and different issues, negligible administration intrusion emerge. To fathom the intrusion, correction procedure is done. In sensible circumstances, fix procedure can't be started promptly because of different reasons. Consequently the idea of defer happens between administration interference and patch up procedure. The lining system improves the system measurements, for example, generally speaking system throughput, lessens the course delay, over hard and traffic blockage likelihood.

\subsection{QUEUING THEORY APPROACH}

The VOD lining issue is as per the following: Clients arriving in batches follows a Poisson system. Service starts and it resumes. It pursues general dissemination and it is rendered in like manner on first started things out served premise in two phases of administration. After the fulfillment of the primary phase of administration, the server takes a crisis vacation. In this course of time, maintenance work for the second phase of administration is done. What's more, server interrupts because of different reason during the season of administration. In continuation, it needs to get into repair process, however here in this circumstance, a delay process idea is been taken over between the intrusion and fix process. Additionally, to augment the up keep work of the framework a mandatory excursion is presented after the culmination of second phase of administration. The idea of Balking assumes a noticeable job in this model. Seeing the line, clients may stop the framework without joining the line. Furthermore, to that, a portion of the customers may leave the line and quit the framework because of eagerness. This procedure is known to renege and it occurs in our model during the postpone time between administration intrusion and redo process. All the characterized parameters pursue a general conveyance. 


\section{The issue is depicted by the concept of birth and death process and utilizing the steady state conditions of the model defined}

VOD application issue is understood by the strategy for beneficial variable method. For every one of the phase of administration, postpone time, fix process, crisis get-away and necessary get-away beneficial variables are used. Enduring state likelihood creating line measure, length of the line, number of clients in the framework, holding up time of the clients in the framework just as in the line are resolved. Likewise, the use factor, the time spent by the server for the administration and inactive time of the server are inferred for the characterized VOD programming issue. Numerical depiction legitimizes the model and the graphical depiction gives a sensible picture about the decisions to be taken before the startup of the organization. To deteriorate the issue in VOD programming, an undeniable endorsement is rendered close to the end, by strategies for looking at the numerical results and graphical examination of the model.

\section{MATHEMATICAL ASSUMPTIONS OF THE MODEL}

Clients arrive in groups for service with mean arrival rate $\lambda^{+}>0$.

The first order probability that a batch of i customers arrives at the system is $\lambda^{+} d_{i}$ Here $0 \leq d_{i} \leq 1$ and $\sum_{i=1}^{n} d_{i}=1$

For the first stage of service, $\theta_{n}^{(1)}(x), \eta_{1}(x)$ is the conditional probability of completion of completion of first stage of service. The probability distribution function of the first stage of service and its corresponding density function are given by $E^{*}(x)$ and $e^{*}(x)$. Hence:

$$
\eta_{1}(x)=\frac{e^{*}(x)}{1-E^{*}(x)}, \quad e^{*}(x)=\eta_{1}(s) e^{-\int_{0}^{s} \eta_{1}(x) d x}
$$

Similarly for all the other parameters, Emergency vacation $\left(M_{n}^{E}(x)\right)$, stage 2 procedure $\left(\theta_{n}^{(2)}(x)\right)$, Compulsory vacation $\left(M_{n}^{(c)}(\mathrm{x})\right)$, Delay process $\left(D_{n}(x)\right)$, Repair process we have the following functions respectively:

$$
\begin{array}{ll}
\delta_{E}(x)=\frac{a^{*}(x)}{1-S^{*}(x)}, & a^{*}(x)=\delta_{E}(s) e^{-f \delta_{E}(x) d x} \\
\delta_{2}(x)=\frac{a_{1}^{*}(x)}{1-h_{1}^{*}(x)}, & a_{1}^{*}(x)=\delta_{2}(s) e^{-\int \delta_{2}(x) d x}
\end{array}
$$




$$
\begin{gathered}
\delta_{c}(x)=\frac{w^{*}(x)}{1-W^{*}(x)}, \quad w^{*}(x)=\delta_{c}(s) e^{-\int_{0}^{s} \delta_{c}(x) d x} \\
\varphi(x)=\frac{a_{2}^{*}(x)}{1-A_{2}^{*}(x)}, \quad a_{2}^{*}(x)=\varphi(s) e^{-\int_{0}^{s} \varphi(x) d x} \\
\gamma(x)=\frac{v^{*}(x)}{1-K^{*}(x)}, \quad v^{*}(x)=\gamma(s) e^{-\int_{0}^{s} \gamma(x) d x}
\end{gathered}
$$

During delay process, the process of reneging takes place. That is the clients leave the system due to impatience with probability $\varepsilon$ after joining the Queue. Also break down occurs during the stages of service with arrival rate $\beta>0$ follows a Poisson distribution. After entering into the system, seeing the Queue, some customers may not join the Queue and they leave the system. This process of Balking occurs with probability b during the time of repair process in this Queuing system.

\section{GOVERNING EQUATIONS OF THE MODEL}

The VOD Queuing model is first defined as a set of difference differential equations:

$$
\begin{aligned}
& \frac{\partial}{\partial x} \theta_{n}^{(1)}(x)+\left(\lambda^{+}+\eta_{1}(x)+\beta\right) \theta_{n}^{(1)}(x)=\lambda^{+} \sum_{i=1}^{n} d_{i} \theta_{n-i}^{(1)}(x) \\
& \frac{\partial}{\partial x} \theta_{0}^{(1)}(x)+\left(\lambda^{+}+\eta_{1}(x)+\beta\right) \theta_{0}^{(1)}(x)=0 \\
& \frac{\partial}{\partial x} M_{n}^{E}(x)+\left(\lambda^{+}+\delta_{E}(x)\right) M_{n}^{E}(x)=\lambda^{+} \sum_{i=1}^{n} d_{i} M_{n-i}^{E}(x) \\
& \frac{\partial}{\partial x} M_{0}^{E}(x)+\left(\lambda^{+}+\delta_{E}(x)\right) M_{0}^{E}(x)=0 \\
& \frac{\partial}{\partial x} \theta_{n}^{(2)}(x)+\left(\lambda^{+}+\eta_{2}(x)+\beta\right) \theta_{n}^{(2)}(x)=\lambda^{+} \sum_{i=1}^{n} d_{i} \theta_{n-i}^{(2)}(x) \\
& \frac{\partial}{\partial x} \theta_{0}^{(2)}(x)+\left(\lambda^{+}+\eta_{2}(x)+\beta\right) \theta_{0}^{(2)}(x)=0 \\
& \frac{\partial}{\partial x} M_{n}^{(c)}(x)+\left(\lambda^{+}+\delta_{c}(x)\right) M_{n}^{(c)}(x)=\lambda^{+} \sum_{i=1}^{n} d_{i} M_{n-i}^{(c)}(x) \\
& \frac{\partial}{\partial x} M_{0}^{(c)}(x)+\left(\lambda^{+}+\delta_{c}(x)\right) M_{0}^{(c)}(x)=0 \\
& \frac{\partial}{\partial x} D_{n}(x)+\left(\lambda^{+}+\varphi(x)+\epsilon\right) D_{n}(x)=\lambda^{+} \sum_{i=1}^{n} d_{i} D_{n-i}(x)+\epsilon D_{n+1}(x) \\
& \frac{\partial}{\partial x} D_{0}(x)+\left(\lambda^{+}+\varphi(x)+\epsilon\right) D_{0}(x)=\epsilon D_{1}(x) \\
& \frac{\partial}{\partial x} k_{n}(x)+\left(\lambda^{+}+\gamma(x)\right) k_{n}(x)=\lambda^{+}(1-b) k_{n}(x)+b \lambda^{+} \sum_{i=1}^{n} d_{i} K_{n-i}(x) \\
& \frac{\partial}{\partial x} k_{0}(x)+\left(\lambda^{+}+\gamma(x)\right) k_{0}(x)=\lambda^{+}(1-b) k_{0}(x) \\
& \lambda^{+} Q=\int_{0}^{\infty} k_{0}(x) \gamma(x) d x+\int_{0}^{\infty} M_{0}^{(c)}(x) \delta_{c}(x) d x+\lambda^{+}(1-b) Q
\end{aligned}
$$




\section{Boundary conditions}

The following boundary conditions are used to solve the above equations:

$$
\begin{gathered}
\theta_{n}^{(1)}(0)=\int_{0}^{\infty} M_{n+1}^{(c)}(x) \delta_{c}(x) d x+\int_{0}^{\infty} R_{n+1}(x) \gamma(x) d x \\
\theta_{n}^{(2)}(0)=\int_{0}^{\infty} M_{n}^{E}(x) \delta_{E}(x) d x+(1-s) \int_{0}^{\infty} \theta_{n}^{(1)}(x) \eta_{1}(x) d x \\
M_{n}^{E}(0)=s \int_{0}^{\infty} \theta_{n}^{(1)}(x) \eta_{1}(x) d x \quad n \geq 0 \\
M_{n}^{(c)}(0)=\int_{0}^{\infty} \theta_{n}^{(2)}(x) \eta_{2}(x) d x \quad n \geq \\
D_{n}(0)=\beta \int_{0}^{\infty} \theta_{n}^{(2)}(x) d x=\beta \theta_{n-1}^{(2)}(x) \quad n \geq 1 \\
k_{n}(0)=\int_{0}^{\infty} D_{n}(x) \varphi(x) d x n \geq 0
\end{gathered}
$$

\section{DISTRIBUTION OF THE QUEUE LENGTH AT ANY POINT OF TIME}

To solve equations (1) to (12) for a closed form solution we follow the procedure set out below.

We multiply (1) by and sum over $\mathrm{x}$ from 1 to and add it to (2)

We get,

$$
\frac{\partial}{\partial x} \theta_{q}^{(1)}(x, z)+\left(\lambda^{+}-\lambda^{+} D(z)+\eta_{1}(x)\right) \theta_{q}^{(1)}(x, z)=0
$$

Similarly,

$$
\begin{gathered}
\frac{\partial}{\partial x} M_{q}^{(E)}(x, z)+\left(\lambda^{+}-\lambda^{+} D(z)+\delta_{E}(x)\right) M_{q}^{(E)}(x, z)=0 \\
\frac{\partial}{\partial x} \theta_{q}^{(2)}(x, z)+\left(\lambda^{+}-\lambda^{+} D(z)+\eta_{2}(x)+\beta\right) \theta_{q}^{(2)}(x, z)=0 \\
\frac{\partial}{\partial x} M_{q}^{(c)}(x, z)+\left(\lambda^{+}-\lambda^{+} D(z)+\delta_{c}(x)\right) M_{q}^{(c)}(x, z)=0 \\
\frac{\partial}{\partial x} D_{q}(x, z)+\left(\lambda^{+}-\lambda^{+} D(z)+\epsilon-\frac{\varepsilon}{z}+\varphi(x)\right) D_{q}(x, z)=0 \\
\frac{\partial}{\partial x} K_{q}(x, z)+\left(b\left(\lambda^{+}-\lambda^{+} D(z)\right)+\gamma(x)\right) K_{q}(x, z)=0
\end{gathered}
$$

Integrating (20)-(25) between limits 0 to $x$, we obtain 


$$
\begin{gathered}
\theta^{(1)}(x, z)=\theta^{(1)}(0, z) \exp \left[-\left(\lambda^{+}-\lambda^{+} D(z)\right) x-\int_{0}^{\infty} \eta_{1}(t) d t\right] \\
M^{E}(x, z)=M^{E}(0, z) \exp \left[-\left(\lambda^{+}-\lambda^{+} D(z)\right) x-\int_{0}^{\infty} \delta_{E}(t) d t\right] \\
\theta^{(2)}(x, z)=\theta^{(2)}(0, z) \exp \left[-\left(\lambda^{+}-\lambda^{+} D(z)+\beta\right) x-\int_{0}^{\infty} \eta_{2}(t) d t\right] \\
M^{(c)}(x, z)=M^{(c)}(0, z) \exp \left[-\left(\lambda^{+}-\lambda^{+} D(z)\right) x-\int_{0}^{\infty} \delta_{c}(t) d t\right] \\
D(x, z)=D(0, z) \exp \left[-\left(\lambda^{+}-\lambda^{+} D(z)+\xi-\frac{\xi}{z}\right) x-\int_{0}^{\infty} \varphi(t) d t\right] \\
K(x, z)=K(0, z) \exp \left[-b\left(\lambda^{+}-\lambda^{+} D(z)\right) x-\int_{0}^{\infty} \gamma(t) d t\right]
\end{gathered}
$$

The above set of equations (26) holds for all $x>0$

We next multiply the boundary conditions by suitable powers of $z^{n}$ and taking summation over all possible values of $n$ and using (13) we get after simplification

$$
\begin{gathered}
z \theta^{(1)}(0, z)=z \lambda^{+} b_{1}(D(z)-1)+\int_{0}^{\infty} M^{(c)}(x, z) \delta_{c}(x) d x+\int_{0}^{\infty} K(x, z) \gamma(x) d x \\
\theta^{(2)}(0, z)=\int_{0}^{\infty} M^{E}(x, z) \delta_{E}(x) d x+(1-s) \int_{0}^{\infty} \theta^{(1)}(x, z) \eta_{1}(x) d x \\
M^{E}(0, z)=s \int_{0}^{\infty} \theta^{(1)}(x, z) \eta_{1}(x) d x \\
M_{n}^{(c)}(0, z)=\int_{0}^{\infty} \theta^{(2)}(x, z) \eta_{2}(x) d x \\
D(0, z)=\beta z \int_{0}^{\infty} \theta^{(2)}(x, z) d x \\
K(0, z)=\int_{0}^{\infty} D(x, z) \varphi(x) d x
\end{gathered}
$$

Integrating (26) by parts with respect to $\mathrm{x}$, we get,

$$
\theta^{(1) *}(z)=\theta^{(1) *}(0, z)\left(\frac{1-E^{*}(g)}{g}\right), g=\lambda^{+}-\lambda^{+} D(z)
$$

Where $E^{*}(g)=\int_{0}^{\infty} e^{-\left(\lambda^{+}-\lambda^{+} D(z)\right) x} d E_{1}(x)$ is the Laplace transform of the service time of first stage.

Again multiplying (26) on both sides by $\eta_{1}(x)$ and integrating over $x$, we get

$$
\int_{0}^{\infty} \theta^{(1) *}(x, z) \eta_{1}(x) d x=\theta^{(1) *}(0, z) E^{*}(g)
$$


Similarly,

$$
\begin{gathered}
\theta^{(2)^{*}}(z)=\theta^{(2) *}(0, z)\left(\frac{1-A_{1}^{*}(h)}{h}\right), h=\lambda^{+}-\lambda^{+} D(z)+\beta \\
\theta^{(2)^{*}}(z)=\theta^{(2) *}(0, z)\left(\frac{1-A_{1}^{*}(h)}{h}\right), h=\lambda^{+}-\lambda^{+} D(z)+\beta \\
\int_{0}^{\infty} \theta^{(2) *}(x, z) \eta_{2}(x) d x=\theta^{(2) *}(0, z) A_{1}^{*}(h) \\
\int_{0}^{E *}(z)=M^{E *}(0, z)\left(\frac{1-S^{*}(g)}{g}\right) \\
M_{0}^{\infty *}(x, z) \delta_{E}(x) d x=M^{(E *)}(0, z) S^{*}(g) \\
M^{(c) *}(z)=M^{(c) *}(0, z)\left(\frac{1-W^{*}(g)}{g}\right) \\
\int_{0}^{\infty} M^{(c) *}(x, z) \delta_{c}(x) d x=M^{(E) *}(0, z) W^{*}(g) \\
D^{*}(z)=D^{*}(0, z)\left(\frac{1-A_{2}^{*}(q)}{q}\right), q=\left(\lambda^{+}-\lambda^{+} K(z)+\xi-\frac{\xi}{z}\right) \\
\int_{0}^{\infty} D^{*}(x, z) \varphi(x) d x=D^{*}(0, z) A_{2}^{*}(q) \\
K^{*}(z)=K^{*}(0, z)\left(\frac{1-K^{*}(b g)}{b g}\right)
\end{gathered}
$$

Now utilizing the above relations (38) in (32)-(37), we get

$$
\begin{gathered}
z \theta^{(1)}(0, z)=z \lambda^{+} b_{1}(D(z)-1)+s W^{*}(g) \theta^{(1) *}(0, z) E^{*}(g)+K^{*}(b g) A_{2}^{*}(q) \beta z\left(\frac{1-A_{1}^{*}(h)}{h}\right)+\left[\left(\frac{1-S^{*}(g)}{g}\right) s \theta^{(1) *}(0, z) E^{*}(g)+(1-s) \theta^{(1) *}\right. \\
\theta^{(1)}(0, z)=\frac{z \lambda^{+} b_{1}(D(Z)-1)}{z-s W^{*}(g) E^{*}(g)-K^{*}(b g) A_{2}^{*}(q) \beta z\left(\frac{1-A_{1}^{*}(h)}{h}\right)\left[\frac{1-S^{*}(g)}{g} s+(1-s)\right] E^{*}(g)}
\end{gathered}
$$

Hence we get the following from (36) using (39) 


$$
\begin{aligned}
& \theta^{(1) *}(z)=\frac{z \lambda^{*} b_{1}(D(Z)-1)}{z-s W^{*}(g) E^{*}(g)-K^{*}(b g) A_{2}^{*}(q) \beta z\left(\frac{1-\alpha^{*}(k)}{h}\right)\left[\frac{1-s^{*}(g)}{g} s+(1-s)\right] E^{*}(g)}\left[\frac{1-E^{*}(g)}{g}\right] \\
& \theta^{(2) *}(z)=\left[S^{*}(g) s+(1-s)\right] E^{*}(g) \frac{z \lambda^{*} b_{1}(D(Z)-1)\left(\frac{1-A_{j}^{*}(h)}{h}\right)}{\left.z-s W^{*}(g) E^{*}(g)-K^{*}(b)\right) A_{2}^{*}(q) \beta z\left(\frac{1-A_{h}^{*}(h)}{h}\right)\left[\frac{1-s^{*}(g)}{g} s+(1-s)\right] E^{*}(g)} \\
& M^{E^{*}}(z)=s E^{*}(g)\left(\frac{1-s^{*}(g)}{g}\right) \frac{z \lambda^{*} b_{1}(D(L)-1)}{z-s W^{*}(g) E^{*}(g)-K^{*}(b g) A_{2}^{*}(q) \beta z\left(\frac{1-1 \phi^{*}(k)}{h}\right)\left[\frac{1-s^{*}(g)}{g} s+(1-s)\right] E^{*}(g)} \\
& M^{(c) *}(z)=A_{1}^{*}(h)\left[S^{*}(g) s+(1-s)\right] E^{*}(g) \frac{z \lambda^{+} b_{1}(D(Z)-1)\left(\frac{1-W^{*}(g)}{g}\right)}{z-s W^{*}(g) E^{*}(g)-K^{*}(b g) A_{2}^{*}(q) \beta z\left(\frac{1-A_{1}^{*}(h)}{h}\right)\left[\frac{1-S^{*}(g)}{g} s+(1-s)\right] E^{*}(g)} \\
& D^{*}(z)=\beta z\left(\frac{1-A_{1}^{*}(h)}{h}\right)\left(\frac{1-A_{2}^{*}(q)}{q}\right) \frac{z \lambda^{*} b_{1}(D(Z)-1)\left[S^{*}(g) s+(1-s)\right] E^{*}(g)}{z-s W^{*}(g) E^{*}(g)-K^{*}(b g) A_{2}^{*}(q) \beta z\left(\frac{1-A^{*} * h}{h}\right)\left[\frac{1-s^{*}(g)}{g} s+(1-s)\right] E^{*}(g)} \\
& K^{*}(z)=A_{2}^{*}(q) \beta z\left(\frac{1-A_{1}^{*}(h)}{h}\right)\left[S^{*}(g) s+(1-s)\right] E^{*}(g) \frac{z \lambda^{+} b_{1}(D(Z)-1)\left(\frac{1-K^{*}(b g)}{b g}\right)}{z-s W^{*}(g) E^{*}(g)-K^{*}(b g) A_{2}^{*}(q) \beta z\left(\frac{1-A_{1}^{*}(h)}{h}\right)\left[\frac{1-S^{*}(g)}{g} s+(1-s)\right]}
\end{aligned}
$$

\section{PROBABILITY GENERATING FUNCTION OF THE QUEUE SIZE}

To find the probability generating function of the queue size,

Let, $T_{q}(z)=\theta^{(1) *}(z)+\theta^{(2) *}(z)+M^{E *}(z)+M^{(c) *}(z)+D^{*}(z)+K^{*}(z)$

$\frac{-z b(1-E(g))+S(g) s+(1-s)] E^{*}(g) z \lambda^{*} b_{1}(D(Z)-1)\left(\frac{1-A^{*}(h)}{h}\right)-z b E^{*}(g)\left[1-S^{*}(g)\right] s-z b A_{1}^{*}(h)\left[S^{*}(g) s+(1-s)\right] E^{*}(g)\left(1-W^{*}(g)\right)+\beta z z^{*}(g) z b \lambda^{+}(D(Z)-1)\left(\frac{1-A^{*}(h)}{h}\right)\left(\frac{1-A_{2}^{*}(q)}{q}\right)\left[S^{*}(g) s+(1-s)\right]-A_{2}^{*}}{\left.z-s W^{*}(g) E^{*}(g)-K^{*}(b g) A_{2}^{*}(q)\right] z\left(\frac{1-A^{*}(k)}{h}\right)\left[\frac{\left[-S^{*}(g)\right.}{g} s+(1-s)\right] E^{*}(g)}$

\section{IDLE TIME AND UTILIZATION FACTOR}

The normalization condition $T_{q}(1)+Q=1$ is used in order to determine $Q$. Because of the indetermine of $T_{q}(1)$, L'Hopital's rule is applied in (40) to achieve

$$
T_{q}(z)=\frac{N^{\prime}(1)}{D^{\prime}(1)}
$$

Now adding $Q$ to $T_{q}(z)$ given in equation (41) and equating to 1 and simplifying we obtain

$$
Q=\frac{D^{\prime}(1)}{D^{\prime}(1)+N^{\prime}(1)}
$$

Mean length of the Queue and to find $L_{q}$, the steady state average queue length, where

$$
L_{q}=\frac{d}{d z} T_{q}(z) \mid z=1
$$

We note that this formula is of $\frac{0}{0}$ form. 
Hence we write $T_{q}(z)$ as $T_{q}(z)=\frac{N(z)}{D(z)}$ where $\mathrm{N}(\mathrm{z})$ and $\mathrm{D}(\mathrm{z})$ are the numerator and denominator of equation (40)

Then using L'Hopital's rule, we obtain

$$
L_{q}=\lim _{z \rightarrow 1} \frac{D^{\prime}(z) N^{\prime \prime}(z)-N^{\prime}(z) D^{\prime \prime}(z)}{2\left(D^{\prime}(z)\right)^{2}}
$$

Finding the required derivatives at $\mathrm{z}=1$, we have

$$
\begin{gathered}
N^{\prime}(1)=b_{1}\left\{\left(-b_{1} E(R) \lambda^{+}+1\right)\left(\frac{1-A_{i}^{*}(\beta)}{\beta}\right)+E(W) \lambda^{+}\right\}-b_{1}\left[\lambda^{+}+\lambda^{+} s E(s)\right] \\
D^{\prime}(1)=1+s \lambda^{+}[E(L)+E(W)]-\left(1-A_{1}^{*}(\beta)\right)(1-s)\left\{\left[\left(b E(R) \lambda^{+}+R^{*}(b) E(L)\right)\left(\lambda^{+}\right)\right]+\left[-E\left(A_{2}\right)\left(-\lambda^{+}+\xi\right)+1\right] R^{*}(b)\right\}-R^{*}(b) \\
N^{\prime \prime}(1)=\left(2 b_{1} s \lambda^{+} E(s)+2 b_{1} E(L) \lambda^{+}+2 b_{1}\right)\left[\left(-\lambda^{+} b_{1} E(R)+\lambda^{+}\right)\left(\frac{1-A_{i}^{*} \beta}{\beta}\right)+E(W) \lambda^{+}\right]+\left[b_{1} \frac{\beta}{b_{1}} E(R)+\left(-\lambda^{+}+\xi\right)\left(-\lambda^{+}\right) E(R)+\lambda^{+} \beta\right. \\
D^{\prime \prime}(1)=-s\left(\lambda^{+}\right)^{2}\left[2 E(W) E(L)+E\left(L^{2}\right)+E\left(W^{2}\right)\right]-b_{1}\left(\lambda^{+}\right)^{2} E\left(R^{2}\right)\left(1-A_{1}^{*}(\beta)\right)(1-s)+E\left(A_{2}\right)\left(\lambda^{+}\right)^{2} E(R)\left(1-A_{1}^{*}(\beta)\right)(1-s)+E(R) b
\end{gathered}
$$

Substituting (44) in (43) we obtain $L_{q}$ in closed form.

Further, the man waiting time of the customers in the queue as well as in the system and number of customers waiting in the system can be found using Little's law

$$
W_{q}=\frac{L_{q}}{\lambda^{+}}, \mathrm{W}=\frac{L}{\lambda^{+}}, \mathrm{L}=L_{q}+\rho
$$

\section{NUMERICAL JUSTIFICATION OF THE MODEL}

Assume that service time follows exponential distribution in particular and based on this condition, the numerical justification is elaborated below. The values are collected accordingly: $\lambda^{+}=3, \beta=4, b_{1}=1, \varphi=3, \gamma=2.5, \mathrm{~s}=0.5, \eta_{1}=4, \eta_{2}=5, \delta_{E}=3.5, \delta_{c}=2$

$$
\begin{aligned}
& E(L)=\frac{1}{\eta_{1}}, E\left(L^{2}\right)=\frac{2}{\eta_{1}}, E(s)=\frac{1}{\delta_{E}}, E\left(s^{2}\right)=\frac{2}{\delta_{E^{2}}}, \quad E(W)=\frac{1}{\delta_{c}}, \quad E\left(W^{2}\right)=\frac{2}{\delta_{c}^{2}}, \quad A_{1}^{\prime}(\beta)=\frac{-\eta_{2}}{\left(\eta_{2}+\beta\right)^{2}}, \\
& E\left(A_{2}\right)=\frac{1}{\varphi}, E\left(A_{2}^{2}\right)=\frac{2}{\varphi^{2}}, E(R)=\frac{1}{\gamma}, E\left(R^{2}\right)=\frac{2}{\gamma^{2}}, R^{*}(b)=1, A_{1}^{*}(\beta)=\frac{\eta_{2}}{\eta_{2}+\beta}
\end{aligned}
$$

Table 1. Effect Of ChangeOf Reneging ( $\xi=1,1.5,2,2.5,3)$.

\begin{tabular}{|c|c|c|c|c|c|}
\hline $\boldsymbol{Q}$ & $\boldsymbol{\rho}$ & $\boldsymbol{L}$ & $\boldsymbol{L}$ & $\boldsymbol{W}_{\boldsymbol{q}}$ & $\boldsymbol{W}$ \\
\hline 0.4116 & 0.5884 & 7.9348 & 8.5232 & 2.8411 & 2.6449 \\
\hline 0.4197 & 0.5803 & 7.4296 & 8.0099 & 2.67 & 2.4765 \\
\hline 0.4275 & 0.5725 & 6.9683 & 7.5408 & 2.5136 & 2.3228 \\
\hline 0.4352 & 0.5648 & 6.5741 & 7.1389 & 2.3796 & 2.1914 \\
\hline 0.4426 & 0.5574 & 6.1635 & 6.7209 & 2.2403 & 2.0545 \\
\hline
\end{tabular}




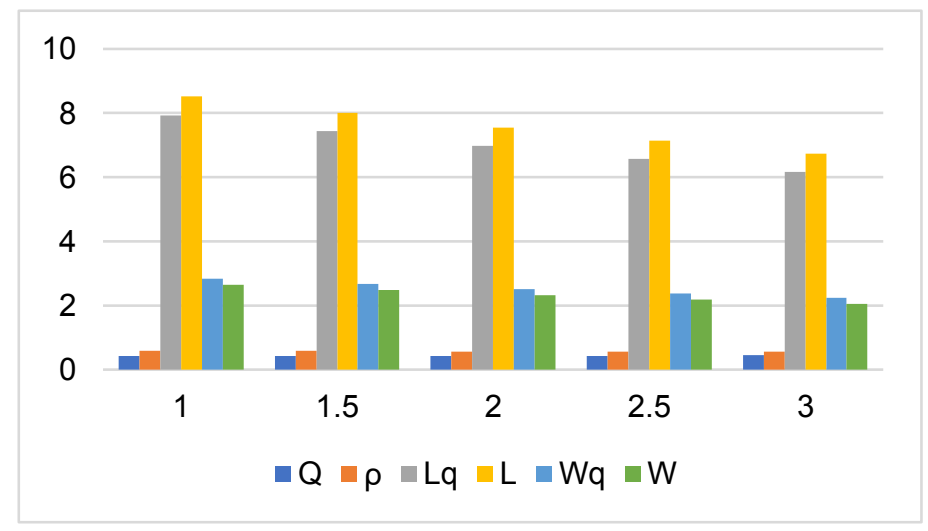

Graphic 1. Effect of change of Reneging.

Table 2. Effect Of Change Of Breakdown ( $\beta=1.5,2,2.5,3,3.5)$.

\begin{tabular}{|c|c|c|c|c|c|}
\hline$Q$ & $\rho$ & $L_{q}$ & $L$ & $W_{Q}$ & $W$ \\
\hline 0.4705 & 0.5295 & 2.1088 & 2.6383 & 0.7029 & 0.8794 \\
\hline 0.4537 & 0.5463 & 2.7691 & 3.3154 & 0.9230 & 1.1051 \\
\hline 0.4484 & 0.5516 & 3.1270 & 3.6786 & 1.0423 & 1.2262 \\
\hline 0.4209 & 0.5791 & 4.0008 & 4.5799 & 1.3336 & 1.5266 \\
\hline 0.4141 & 0.5859 & 4.4935 & 5.0794 & 1.4978 & 1.6931 \\
\hline
\end{tabular}

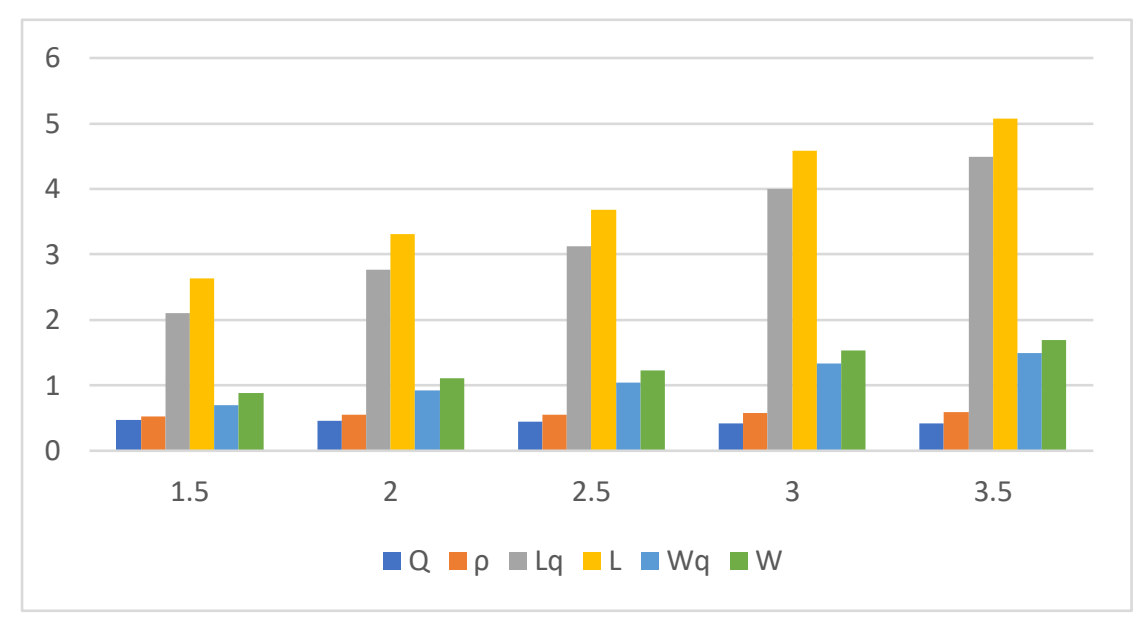

Graphic 2. Effect of change of Breakdown.

From the Table 1, the fact is clear that, as the service goes on in the system, the process of reneging factor occurring during delay process increases. This creates an effect in all the Queue execution measures. It leads to an increase in the idle time and hence the utilization 
factor decreases. Length of the Queue, number of customers in the system and the waiting time gets decreased.

Next, Table 2 shows the performance measures of the system when the break down factor increases. It leads to an increase in all the performance measures of the system as expected. The Idle time dropped off and it leads to an enlarge the utilization factor.

\section{CONCLUSION}

This VOD application process clearly defines the Queuing model consisting of the parameters Stages of service, multi vacation policy, Delay process, service interruption, revamp process, Balking and Reneging. VOD service is well analyzed by means of Queuing approach and the problem is solved by supplementary variable method. Queue performance measures are derived and the model is well justified by the way of numerical illustration. All the results are as expected. 


\section{REFERENCES}

Abeywickrama, S., \& Wong, E. (2013). Delivery of video-on-demand services using local storages within passive optical networks. Optics Express, 21(2), 2083-2096. https://doi. org/10.1364/OE.21.002083

Alomari, S. A., \& Sumari, P. (2011). Statistical information of the Increased demand for watch the VOD with theincreased sophistication in the mobile devices,communications and internet penetration in Asia. The International fournal of Multimedia and its Applications, 3(4), 183-199. https://arxiv.org/ftp/arxiv/papers/1112/1112.2042.pdf

Ghimire, S., Ghimire, R., Thapa, G. B., \& Fernandes, S. (2017). Multi-server batch service queuing model with variable service rates. International fournal of Applied Mathematics and Statistical Science, 6(4), 43-54. https://www.researchgate.net/ publication/321 135050_MULTI-SERVER_BATCH_SERVICE_QUEUEING_ MODEL_WITH_VARIABLE_SERVICE_RATES

Gupta, S. (2013). IPTV: A new dimension in online video streaming - A study of the Indian scenario. Global Media Fournal, 4(1), 1-17. https://www.caluniv.ac.in/globalmdia-journal/CMENT_JUNE_2013/COM-1-Sourav.pdf

He, Y., \& Liu, Y. (2009). VOVO: VGR-oriented Video-on-Demand in Large scale peerto-peer networks. IEEE Transactions on Parallel and Distributed systems, 20(4), 528-539. https://doi.org/10.1109/TPDS.2008.102

Jain, M., \& Bhargava, G. (2008). Bulk arrival retrial queue with unreliable server and priority subscribers. International Fournal of Operational Research, 5(4), 242-259. https:// www.researchgate.net/publication/266828621_Bulk_Arrival_Retrial_Queue_ with_Unreliable_Server_and_Priority_Subscribers

Juhn, L. S., \& Tseng, L. M. (1998). Fast Data broadcasting and receiving scheme for popular video service. IEEE Transactions on Broadcasting, 44(1), 100-105. https://doi. org/10.1109/11.713059 
Kanrar, S. (2012). Analysis and implementation of the large scale video-on-demand system. International Journal of Applied Information Systems, 2(12), 1-9. https://arxiv.org/ $\mathrm{ftp} /$ arxiv/papers/1202/1202.5094.pdf

Maragathasundari, S. (2015). A Bulk arrival queuing model of three stages of service with different vacation policies service interruption and delay time. American International Journal of Research in Science and Technology, 11(1), 52-56. https://www.researchgate. net/publication/295605742_A_bulk_arrival_queueing_model_of_three_stages_of_ service_with_different_vacation_policies_service_interruption_and_delay_time

Maragathasundari, S., \& Karthikeyan, K. (2016). A Bulk queuing model of Optional second phase service with short and long vacations. International fournal of Scientific Research in Science and Technology, 2(5), 196-201. https://www.academia. edu/29971131/A_Bulk_Queuing_Model_of_Optional_Second_Phase_Service_ with_Short_and_Long_Vacations

Maragathasundari, S., Anandapriya, B., Gothaiammal, S. B., \& Gowri, V. (2017). M/G/1 Queue with restricted availability during service interruption and compulsory vacation of deterministic time. International Fournal of Mathematics Trends and Technology, 52(1), 5-9. http://www.ijmttjournal.org/2017/Volume-52/number-1/IJMTTV52P502.pdf

Maragathasundari, S., Srinivasan, S., \& Ranjitham, A. (2014). Analysis of a batch arrival queue with two stages of service and phase vacation. Missouri fournal of Mathematical Sciences, 26(2), 189-205. https://doi.org/10.35834/mjms/1418931959

McManus, J. M., \& Ross, K. W. (1996). Video-on-demand over ATM: Constant-rate transmission and transport. IEEE Journal on selected areas in Communication, 14(6), 10871098. https://doi.org/10.1109/49.508280

Singh Walia, A. (2016). Live multimedia streaming and video on demand issues and challenges. International Journal of Research in Engineering and Technology, 5(2), 91-96. https://www.academia.edu/25603526/LIVE_MULTIMEDIA_STREAMING_ AND_VIDEO_ON_DEMAND_ISSUES_AND_CHALLENGES 
Van Den Broeck, W., Pierson, J., \& Lievens, B. (2007). Video-On-Demand: Towards New Viewing Practices? Observatorio Fournal, 3, 23-44. http://citeseerx.ist.psu.edu/ viewdoc/download?doi=10.1.1.1011.3016\&rep=rep 1\&type=pdf

Viswanathan, S., \& Imielinski, T. (1996). Metropolitan area video-on-demand service using pyramid broadcasting. Multimedia Systems, 4(4), 197-208. https://doi. org/10.1007/s005300050023 


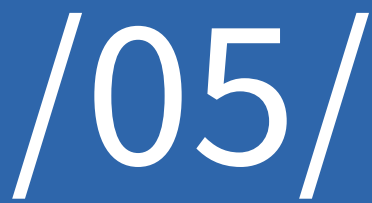




\section{A STUDY ON STAGES OF QUEUING SYSTEM IN AIRCRAFT CONTROL SYSTEM}

S. Maragathasundari

Associate professor, Department of Mathematics, Kalasalingam Academy of Research and Education.

Krishnankoil, (India).

E-mail: maragatham01@gmail.com ORCID: https://orcid.org/0000-0003-1210-6411

C. Prabhu

Assistant professor, Department of Mathematics, Kalasalingam Academy of Research and Education.

Krishnankoil, (India).

E-mail: cprabhumath4@gmail.com ORCID: https://orcid.org/0000-0003-3879-3299

Manikandan Palanivel

Assistant professor, Department of Electronics and Communication Engineering, Kalasalingam Academy of Research and Education.

Krishnankoil, (India).

E-mail: maanip85@gmail.com ORCID: https://orcid.org/0000-0002-5737-0235

Recepción: 05/12/2019 Aceptación: 15/01/2020 Publicación: 23/03/2020

\section{Citación sugerida:}

Maragathasundari, S., Prabhu, C., y Palanivel, M. (2020). A study on stages of queuing system in aircraft control system. 3C Tecnología. Glosas de innovación aplicadas a la pyme. Edición Especial, Marzo 2020, 91-111. http://doi.org/10.17993/3ctecno.2020.specialissue4.91-111

\section{Suggested citation:}

Maragathasundari, S., Prabhu, C., \& Palanivel, M. (2020). A study on stages of queuing system in aircraft control system. 3C Tecnología. Glosas de innovación aplicadas a la pyme. Edición Especial, Marzo 2020, 91-111. http://doi.org/10.17993/3ctecno.2020.specialissue4.91-111 


\section{ABSTRACT}

This inspection looks into a Non Markovian covering issues in which the organization is rendered in two stages. Customers meet up in bunches seeks after a Poisson assignment. Organization time seeks after a general scattering. After the fulfillment of the second period of organization, the server goes for a required vacation, if in need the server has the decision go take a comprehensive trip. Here vacation in the sense addresses the period in the midst of which the help attempts to be improving the state of the server. In like manner, service blocks carelessly in the midst of the period of organization. Everything considered, conditions, it is unavoidable. In this coating issue, separation occurs in the midst of the second period of organization and acknowledged to occur according to a Poisson stream. Similarly, to dodge the blockage, in the midst of the period of optional expanded vacation, a stand by the server is given. At whatever point the server meddles with, it is sent to fix process quickly promptly. For the above portrayed Queuing model, by strategies for the fortifying variable framework, we get the persisting state results in express and close to respect the probability creating capacities with respect to the amount of customers in the line, the typical number of customers, idle time of the server, use factor and the ordinary holding up time in the line. Numerical portrayal and agrow graphical examination are done toward the end to favor the model.

\section{KEYWORDS}

Non-markovianqueue, Optional extended vacation, Queuing performance measures. 


\section{INTRODUCTION}

Queuing speculation is the numerical examination of holding up lines or Queues. It is a basic piece of Mathematics with associated likelihood, quantifiable spread, examination, matrix theory and complex examination. It furthermore falls under the locale of choice hypothesis. It is a practical circumstance that the server is inaccessible to serve the clients amid intermittent timeframes. The period for which the server is inaccessible to serve the clients as indicated by a known or obscure calendar is characterized to be the server getaway period. In lining, writing, the term get-away alludes to a length in which upkeep work to be completed by the server. Doshi (1986) made a broad review on line frameworks with getaways. Madan (2000) examined two stage heterogeneous administrations with the Bernoulli excursion lining model. The same work was later proposed by Keilson and Servi (1987) under certain modifications. Madan and Abu Al-Rub (2004) contemplates the staged kind server getaways base on single get-away approach. The single entry lining framework M/G/1 have been examined by various creators, as Madan and Baklizi (2002), Artalejo and Choudhury (2004) and so on because of its wide applications.All the more as of late, the vast majority of the investigations have been dedicated to group landing excursion models under various get-away approaches due to its interdisciplinary character. Numerous scientists have considered cluster entry lines with get-away time, weeludethe peruse to Altman and Yechiali (2006), Lee, Lee and Chae (1994). In later years a lot of work has been done on clump entry lines with getaways and arbitrary breakdowns. Maragathasundari and Balamurugan (2015) have considered a cluster entry line of administration in two phases with a Bernoulli plan get-away pursued by an all-encompassing get-away and benefit interference. Maragathasundari and Dhanalakshmi (2018) made a coating approach in Mobile adhoc frameworks. Maragathasundari and Srinivasan (2012) investigated non Markovian feedback queue with multiple server vacation. Multistage cluster arrival queue with service interruption have been well analyzed by Maragathasundari and Srinivasan (2015). Discretionary services are well studied by Maragathasundari and Srinivasan (2017) in a Non Markovian Queue. Maraghi, Madan and Darby-Dowman (2010) made an analysis over second discretionary service 


\section{APPLICATION OF THE MODEL DEFINED}

The queuing model what we implemented here is very much suitable for aircrafts scheduling done to the air traffic controllers. The air traffic controller can serve in the form of first come first serve basis. But the aircrafts what we considered here as the customers are arriving as the batches. The air traffic controller provides various services to maintain safe and secure journey provided to the travelers.

\subsection{AIR TRAFFIC CONTROL SYSTEM}

The errand of guaranteeing safe tasks of business and private flying machine falls on air traffic controllers. They should facilitate the developments of thousands of flying machines, keep them at safe separations from one another, immediate them amid departing and arriving from air terminals guide them around awful climate and guarantee that traffic streams easily with negligible postponements. In this article, we will inspect airport regulation in the United States. We'll pursue a departure from takeoff to landing, taking a gander at the different controllers included, what everyone does, the gear they use and how they are prepared.

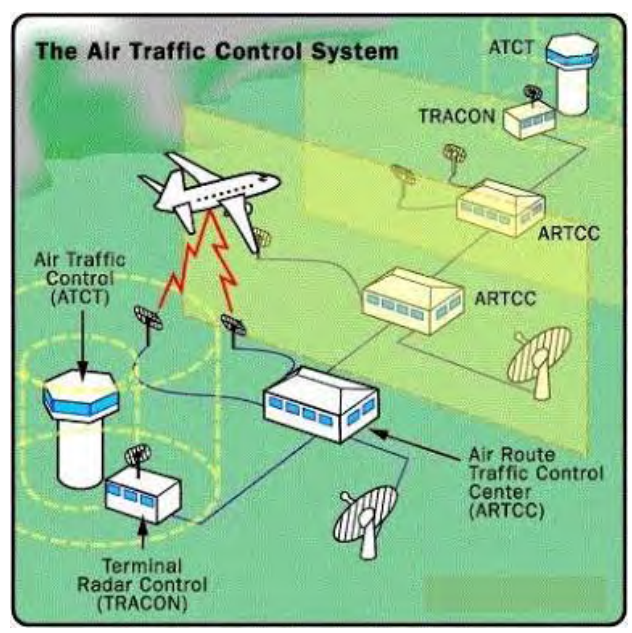

Figure 1. The Air traffic control system.

\subsection{AIR TRAFFIC SERVICES}

Air Traffic Services conveys sheltered, secure, and successful administration for the National Airspace System and worldwide airspace assigned to U.S. control. We are liable for Airport 
Traffic Control Towers (Federal and Contract), Terminal Radar Approach Control offices, Air Route Traffic Control Centers, and Combined Control Facilities to direct flying machine through their different periods of flight. Air traffic controllers at every office are kept up by various specialists, for example, meteorologists, traffic the executive specialists, and $\mathrm{AF}$ administrators who keep up and fix the hardware substructure of the ATC framework.

In this work, we are thinking about ATC, Air Traffic Controller as the server for giving administrations to the arriving Aircrafts. ATC fuses show and control supports, this foundation incorporate $\mathrm{PC}$ frameworks of changing vintages, complex voice and information exchanging gear, radio and microwave transmission frameworks, neighborhood and remote found radio and radar frameworks, and also ecological and electric power molding and reinforcement frameworks, which are required by the hardware.

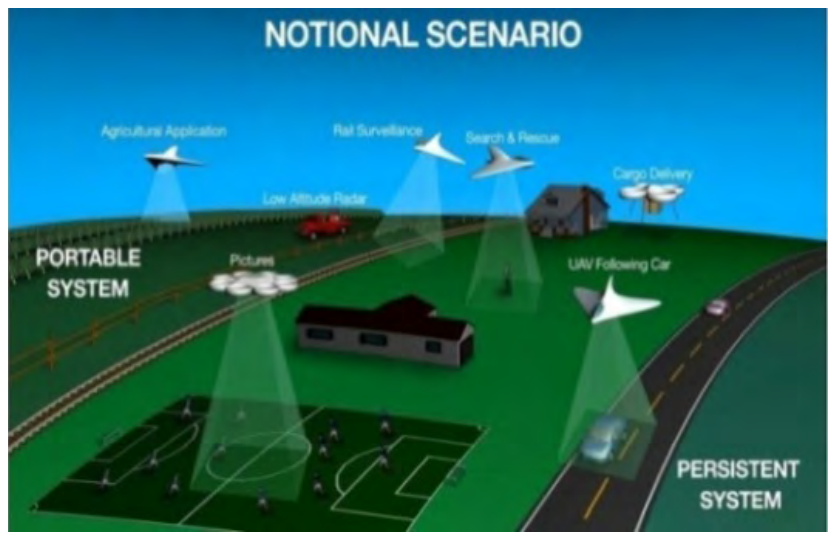

Figure 2. The Air traffic services.

All the parameters defined in the queuing problem are well explained in the application. They are as follows.

\subsection{MULTI ESSENTIAL SERVICES (STAGE 1)}

\section{STAGE I}

Flight data benefit, which offers data helpful for the sheltered and successful holding of flights; the pilot can get data about the landing and takeoff timings of different flights. They need to mindful of the climate investigation report of every single moment send by the radar frameworks. 


\section{STAGE II}

Flight data benefit, which offers data helpful for the sheltered and successful hold Alerting administration, which bears administrations to all known air ships. The pilots will get the explicit data with respect to hazardous situations. On the off chance that there is no such occurrence, it used to send one leeway message to all the air create for further procedure.

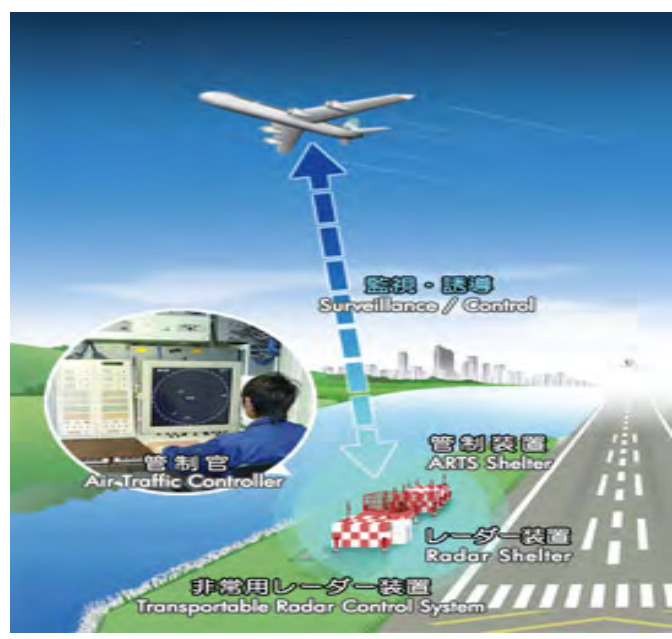

Figure 3. Alerting Administration.

\subsection{INTERRUPTION OCCURRENCE}

Air traffic controllers at every office are bolstered by various pros, for example, meteorologists, traffic the executive's experts. Subsequently, if there any correspondence connects mistake between these pros may cause enormous harm/issue to the flying machines. The showcase and control comforts utilized by controllers probably won't be at the great condition. This framework incorporates PC frameworks of changing vintages, complex voice and information exchanging gear, radio and microwave transmission frameworks, nearby and remote-found radio and radar frameworks, and also there might be a few issues in ecology and electric power molding and reinforcement frameworks, which are required by the hardware.

\subsection{REPAIR WORKS CARRIED OUT IN REPAIR PROCESS}

Repair of aging display computers. The replacement of frequency of outages involving the aging IBM 9020E display channel complex equipment. Replacement of Other recent 
equipment outages involving power systems and communications equipment. To rectify Lack of controller proficiency with the direct access radar channel (DARG) /Standalone mode of the backup computer system. Software error can be rectified by debugging the prolonged coding. Hardware repairs can be rectified by the replacement of some good conditioned equipment. Re alters the wiring connections, cables to avoid outages. Some equipment reassembly may also take place at this stage.

\subsection{COMPULSORY VACATION (REGULAR MAINTENANCE WORK)}

Any time the system is functioning with failed elements, technicians are working to make emergency repairs. Often, technicians work around the clock until repairs are complete. This restricts their ability to comprehend monotonous maintenance tasks and less-serious repairs. The Safety Boardconfirmed three basic, recurring problems distressing air route facilities: (1) Thewell-publicized age of the kit, predominantly the IBM 9020E computer and some related modules, contributes to the existing difficulties. It is hard to maintain, old system with brittle wiring, thousands of difficult-to-repair special circuit boards, and a nearly total lack of direct manufacturer support. Whenever the framework is working with fizzled components, professionals are attempting to make crisis fixes. Frequently, experts work nonstop until the point when fixes are finished. This confines their capacity to complete repetitive support undertakings and less-genuine fixes. The Safety Board affirmed three essential, repeating issues troubling air course offices:

The all-around broadcasted age of the unit, dominatingly the IBM 9020E PC and some related modules, adds to the current challenges. It is difficult to keep up, old framework with fragile wiring, a large number of hard to-fix exceptional circuit sheets, and an about the aggregate absence of direct maker bolster.

Facility reinforcement control hand-off frameworks are additionally intensifying at an expanding rate. This is by all accounts a more critical issue than 9020E disappointments, since it disturbs all electrically fueled frameworks in an office. Communication joins both radio correspondences to airplane and landline interchanges between ATC offices, are likewise confronting the issues at a rate that worries both the Safety Board and the FAA. 


\subsection{OPTIONAL EXTENDED VACATION}

Whenever the framework is working with fizzled components, experts are attempting to make crisis fixes. Regularly, specialists work nonstop until the point that fixes are finished. This confines their capacity to far reaching dull upkeep errands and less-genuine fixes. The Safety Board affirmed three fundamental, repeating issues troubling air course offices: 1) the very much exposed age of the unit, dominatingly the IBM 9020E PC and some related modules, add to the current challenges. It is difficult to keep up, old framework with fragile wiring, a large number of hard to-fix exceptional circuit sheets, and an almost add up to absence of direct producer bolster. 2) Facility reinforcement control transfer frameworks are additionally compounding at an expanding rate.

\subsection{STANDBY SERVER DURING EXTENDED VACATION}

In the event that either the Host framework or the presentation PC flops (or if both come up short), controllers must rely upon on DARC. In case of a presentation PC blackout, DARC passes on related radar and flight plan data to controller PVDs trusting on the Host to movement flight plan data. This method of activity is alluded to as "DARC/Host," and is like task under the essential framework; be that as it may, a few structures are not realistic to controllers. Five structures that are not available amid DARC/Host activity include:

Conflict alarm, a PC undermining that protected flying machine partition has been endangered. Minimum safe height cautioning (MSAW), a PC cautioning that a flying machine is working underneath a preset least elevation. Mode-C interloper alarm, a PC cautioning that an unmanaged airplane is working in the airspace. Remove reference pointer, a moving 5-mile ring around flying machine focuses on that is utilized as a partition helper.

Any time the system is functioning with failed elements, technicians are working to make emergency repairs. Often, technicians work around the clock until repairs are complete. This restricts their ability to comprehend monotonous maintenance tasks and less-serious repairs. The Safety Board confirmed three basic, recurring problems distressing air route facilities: (1) The well-publicized age of the kit, predominantly the IBM 9020E computer and some related modules, contributes to the existing difficulties. It is hard to maintain, 
old system with brittle wiring, thousands of difficult-to-repair special circuit boards, and a nearly total lack of direct manufacturer support. Whenever the framework is working with fizzled components, professionals are attempting to make crisis fixes. Frequently, experts work nonstop until the point when fixes are finished. This confines their capacity to complete repetitive support undertakings and less-genuine fixes. The Safety Board affirmed three essential, repeating issues troubling air course offices:

\section{QUEUEING THEORY APPROACH TO AIRCRAFT SERVICE PROBLEM}

The above characterized Queuing issue (Air creates issue) is disentangled by using the supplementary variable technique. For every one of the organization time, remain by server escape time and fix time valuable variables are used. Unwavering state line gauge dispersal and the different execution measure are settled. Numeral outline legitimizes the model and the graphical depiction gives a sensible picture about the decisions to be taken before the startup of the organization. To deteriorate the issue of clog in the air make benefits, an obvious endorsement is rendered toward the end, by strategies for looking at the numerical results and graphical examination of the model.

\section{NON MARKOVIANQUEUEING PROBLEM}

The Queuing issue is as per the following: This examination researches a non Markovian lining issue in which the administration is rendered in two phases. Clients touch base in clumps pursues a Poisson appropriation. Administration time pursues a general dissemination. After the consummation of the second phase of administration, the server goes for a mandatory get-away, if in need the server has the choice go take an all-encompassing excursion. Here excursion in the sense speaks to the period amid which the support works to be done in the server. Likewise, benefit hinders aimlessly amid the season of administration. All things considered, circumstances, it is unavoidable. In this lining issue, separately happens amid the second phase of administration and accepted to happen as per a Poisson stream. Likewise, to evade the blockage, amid the season of discretionary broadened get-away, a remain by the server is given. At whatever point the server intrudes on, it is sent to fix process promptly immediately. For the above characterized Queuing model, by methods for strengthening 
the variable system, we acquire the enduring state results in express and shout for regarding the likelihood producing capacities for the quantity of clients approve the model. It gives a reasonable pondered the theoretical investigation of Queuing hypothesis.

\section{SCIENTIFIC DEPICTION OF THE MODEL}

Consumers enter in clusters pursues a Poisson procedure. Let $\lambda p_{i} d t$, i varies from 1 to $\mathrm{n}$, is the probability value which denotes the arrival of $\mathrm{i}$ customers in cluster. Here $0 \leq p_{i} \leq 1$ and $\sum_{i=1}^{n} p_{i}=1$ and $\lambda>0$ is the mean landing rate of the batch. Service time follows general distribution. $\int_{0}^{\infty} S_{n}^{(a)}(x) d x, \int_{0}^{\infty} S_{n}^{(b)}(x) d x, \int_{0}^{\infty} B_{n}^{(1)}(x) d x, \int_{0}^{\infty} B_{n}^{(2)}(x) d x$ and $\int_{0}^{\infty} L_{n}(x) d x$ refers to probability that there are $\mathrm{n}$ customers in the queue and the server is in essential service, optional service, compulsory vacation, optional extended vacation and revamp process respectively. Essential service, optional service, compulsory vacation, optional extended vacation and revamp process follows general distribution with distribution function $Q_{a}(s), Q_{b}(s), R_{q 1}(s), R_{q 2}(\mathrm{~s})$ and $L(s)$ and corresponding density function $q_{a}(s), q_{b}(s), r_{q 1}(s), r_{q 2}(s)$ and $l(s)$ repectively. Also $\xi_{a}(x)$ and $\xi_{b}(x)$ be the contingent likelihood of culmination of first fundamental service and flexible administration amid the interim $(x, x+d x)$, given that the slipped time by time is $\mathrm{x}$, so that $\xi_{a}(x)=\frac{q_{a}(x)}{1-\overline{Q_{a}}(x)}$, $q_{a}(s)=\xi_{a}(s) e^{-\int \xi_{a}(x) d x}$ and $\xi_{b}(x)=\frac{q_{b}(x)}{1-\overline{Q_{b}}(x)}, q_{b}(s)=\xi_{b}(s) e^{-\int^{f} \xi_{b}(x) d x}$. Similarly for compulsory vacation and optional extended vacation, we have $\gamma(x)=\frac{r_{q 1}(x)}{1-R_{q 1}(x)}, r_{q 1}(s)=\gamma(s) e^{-\int_{0}^{f} \gamma(x) d x}$ and $\delta(x)=\frac{r_{q 2}(x)}{1-\bar{R}_{q 2}(x)}, \quad r_{q 2}(s)=\delta(s) e^{-f^{-} \delta(x) d x}$. The concept of standby server acts during the optional extended vacation period and it follows exponential distribution with parameter additionally the fix time (repair time) pursues general dispersion. So $\varepsilon(x)=\frac{l(x)}{1-\bar{L}(x)} \quad, l(s)=\epsilon(s) e^{-\int_{0}^{f} \varepsilon(x) d x}$

\section{"The issue is delineated by birth and death process by techniques for Persevering state conditions."}

\section{GOVERNING EQUATIONS OF THE QUEUEING SYSTEM IN STEADY STATE}

To start with, the generating function in terms of probability is given as follows:

$$
\begin{gathered}
S_{n}^{a}(x, z)=\sum_{n=0}^{\infty} f^{n} S_{n}^{a}(x), S_{n}^{b}(x, z)=\sum_{n=0}^{\infty} f^{n} S_{n}^{b}(x) B_{q}^{(1)}(x, f)=\sum_{n=0}^{\infty} z^{n} B_{n}^{(1)}(x) \\
B_{q}^{(2)}(x, f)=\sum_{n=0}^{\infty} z^{n} B_{n}^{(2)}(x), \mathrm{L}(x, f)=\sum_{n=0}^{\infty} z^{n} L_{n}(x)
\end{gathered}
$$


According to the above assumption and description, the following steady state equations are derived:

$$
\begin{gathered}
\frac{\partial}{\partial x} S_{n}^{(a)}(x)=-\left(\varphi+\xi_{a}(x)\right) S_{n}^{(a)}(x)+\varphi \sum_{j=1}^{n} p_{i} S_{n-j}^{(a)}(x) \\
\frac{\partial}{\partial x} S_{0}^{(a)}(x)+\left(\varphi+\xi_{a}(x)\right) S_{0}^{(a)}(x)=0 \\
\frac{\partial}{\partial x} S_{n}^{(b)}(x)=-\left(\varphi+\xi_{b}(x)+\omega\right) S_{n}^{(b)}(x)+\varphi \sum_{j=1}^{n} p_{i} S_{n-j}^{(b)}(x) \\
\frac{\partial}{\partial x} S_{0}^{(b)}(x)+\left(\varphi+\xi_{b}(x)+\omega\right) S_{0}^{(b)}(x)=0 \\
\frac{\partial}{\partial x} B_{n}^{(1)}(x)+(\varphi+\gamma(x)) B_{n}^{(1)}(x)=\sum_{j=1}^{n} p_{i} B_{n-j}^{(1)}(x) \\
\frac{\partial}{\partial x} B_{0}^{(1)}(x)+(\varphi+\gamma(x)) B_{0}^{(1)}(x)=0 \\
(x)+(\varphi+\delta(x)+\tau) B_{n}^{(2)}(x)=\varphi \sum_{j=1}^{n} p_{i} B_{n-j}^{(2)}(x)+\tau B_{n+1}^{(2)}(x) \\
\frac{\partial}{\partial x} B_{0}^{(2)}(x)+(\varphi+\delta(x)+\tau) B_{0}^{(2)}(x)=\tau B_{1}^{(2)}(x) \\
\frac{\partial}{\partial x} L_{n}(x)+(\varphi+\epsilon(x)) L_{n}(x)=\sum_{j=1}^{n} p_{i} L_{n-j}(x) \\
\frac{\partial}{\partial x} L_{0}(x)+(\varphi+\epsilon(x)) L_{0}(x)=0
\end{gathered}
$$

Boundary conditions:

$$
\begin{gathered}
Q D=\int_{0}^{\infty} L_{0}(x) \varepsilon(x) d x+(1-c) \int_{0}^{\infty} B_{0}^{(1)}(x) \gamma(x) d x+\int_{0}^{\infty} B_{0}^{(2)}(x) \delta(x) d x \\
S_{n}^{(a)}(0)=\int_{0}^{\infty} B_{n+1}^{(2)}(x) \delta(x) d x+\int_{0}^{\infty} L_{n+1}(x) \varepsilon(x) d x+\varphi p_{n+1} D+(1-c) \int_{0}^{\infty} B_{n+1}^{(1)}(x) \gamma(x) d x \\
S_{n}^{(b)}(0)=\int_{0}^{\infty} S_{n}^{(a)}(x) \xi_{a}(x) d x \\
B_{n}^{(1)}(0)=\int_{0}^{\infty} S_{n}^{(b)}(x) \xi_{b}(x) d x, \quad x \geq 0 \\
B_{n}^{(2)}(0)=c \int_{0}^{\infty} B_{n}^{(1)}(x) \gamma(x) d x, \quad x \geq 0
\end{gathered}
$$




$$
L_{n}(0)=\omega \int_{0}^{\infty} S_{n-1}^{(b)}(x) d x=\omega S_{n-1}^{(b)}, \quad L_{0}(0)=0
$$

\section{QUEUE SIZE DISTRIBUTION AT A RANDOM EPOCH}

Usage of Supplementary variable method:

We multiply (1) by $f^{n}$ and sum over $n$ from 1 to $\infty$ and add it to (2) results as follows:

$$
\frac{\partial}{\partial x} S_{q}^{(a)}(x, f)+\left(\varphi-\varphi P(f)+\xi_{a}(x)\right) S_{q}^{(a)}(x, f)=0
$$

Applying the similar procedure for the remaining parameters, we have

$$
\begin{gathered}
\frac{\partial}{\partial x} S_{q}^{(b)}(x, f)+\left(\varphi-\varphi P(f)+\xi_{b}(x)+\omega\right) S_{q}^{(b)}(x, f)=0 \\
\frac{\partial}{\partial x} B_{q}^{(1)}(x, f)+(\varphi-\varphi P(f)+\gamma(x)) B_{q}^{(1)}(x, f)=0 \\
\frac{\partial}{\partial x} B_{q}^{(2)}(x, f)+\left(\varphi-\varphi P(f)+\gamma(x)+\tau-\frac{\tau}{f}\right) B_{q}^{(2)}(x, f)=0 \\
\frac{\partial}{\partial x} L_{q}(x, f)+(\varphi-\varphi P(f)+\epsilon(x)) L_{q}(x, f)=0
\end{gathered}
$$

Multiplying (12a) by $f^{n+1}$ and summing over $n$ from 0 to $\infty$ and using (11), we get

$$
f S_{q}^{(a)}(0, f)=\int_{0}^{\infty} B_{q}^{(2)}(x, f) \delta(x) d x+\int_{0}^{\infty} L_{q}(x, f) \varepsilon(x) d x+(1-c) \int_{0}^{\infty} B_{q}^{(1)}(x, f) \gamma(x) d x+\varphi D(P(z)
$$

To find the R.H.S of (21), we proceed as follows

Next multiply (12b) by $f^{n}$ and summing over $n$ from 0 to $\infty$ to obtain

$$
\begin{gathered}
S_{q}^{(b)}(0, f)=\int_{0}^{\infty} S_{q}^{(a)}(x, f) \xi_{a}(x) d x \\
B_{q}^{(1)}(0, f)=\int_{0}^{\infty} S_{q}^{(b)}(x, f) \xi_{b}(x) d x \\
B_{q}^{(2)}(0, f)=c \int_{0}^{\infty} B_{q}^{(1)}(x, f) \gamma(x) d x \\
L_{q}(0, f)=\omega f \int_{0}^{\infty} S_{q}^{(b)}(x, f) d x=\omega f S_{q}^{(b)}(f)
\end{gathered}
$$

Integrating (16) from 0 tox yields 


$$
S_{q}^{(a)}(x, f)=S_{q}^{(a)}(0, f) e^{-(\varphi-\varphi P(f)) x-\int_{0}^{x} \xi_{a}(t) d t}
$$

Where $S_{q}^{(a)}(0, f)$ is given by $(21)$

Integrating (26) by parts with respect to yields

$$
S_{q}^{(a)}(f)=\frac{S_{q}^{(a)}(0, f)\left(1-Q_{a}(\varphi-\varphi P(f))\right)}{\varphi-\varphi P(f)}
$$
Where $\overline{Q_{a}}=\int_{0}^{\infty} e^{-(\varphi-\varphi P(f)) x} d Q_{a}(x)$ is the Laplace Stieltje's transform of the service time
$Q_{a}(x)$.

Multiply both sides of (26) by $\xi_{a}(x)$ and integrating over $x$ we get

$$
\int_{0}^{\infty} S_{q}^{(a)}(x, f) \xi_{a}(x) d x=S_{q}^{(a)}(0, f) \overline{Q_{a}}(\varphi-\varphi P(f))
$$

Similar procedure to be carried out for $(17)-(20)$, we get

$$
\begin{gathered}
S_{q}^{(b)}(f)=\frac{S_{q}^{(b)}(0, f)\left(1-Q_{b}(\varphi-\varphi P(f)+\omega)\right)}{\varphi-\varphi P(f)+\omega} \\
\int_{0}^{\infty} S_{q}^{(b)}(x, f) \xi_{b}(x) d x=S_{q}^{(a)}(0, f) \overline{Q_{a}}(\varphi-\varphi P(f)) \overline{Q_{b}}(\varphi-\varphi P(f)+\omega)
\end{gathered}
$$

For the process of compulsory vacation,

$$
\begin{gathered}
B_{q}^{(1)}(f)=\frac{B_{q}^{(1)}(0, f)\left(1-R_{q_{1}}(\varphi-\varphi P(f))\right)}{\varphi-\varphi P(f)} \\
\int_{0}^{\infty} B_{q}^{(1)}(x, f) \gamma(x) d x=S_{q}^{(a)}(0, f) \overline{Q_{a}}(\varphi-\varphi P(f)) \overline{Q_{b}}(\varphi-\varphi P(f)+\omega) \overline{R_{q_{1}}}(\varphi-\varphi P(f))
\end{gathered}
$$

For optional extended vacation,

$$
\begin{gathered}
B_{q}^{(2)}(f)=\frac{B_{q}^{(2)}(0, f)\left(1-\overline{R_{q_{2}}}\left(\varphi-\varphi P(f)+\tau-\frac{\tau}{f}\right)\right)}{\varphi-\varphi P(f)+\tau-\frac{\tau}{f}} \\
\int_{0}^{\infty} B_{q}^{(2)}(x, f) \delta(x) d x=c S_{q}^{(a)}(0, f) \overline{Q_{a}}(\varphi-\varphi P(f)) \overline{Q_{b}}(\varphi-\varphi P(f)+\omega) \overline{R_{q_{1}}}(\varphi-\varphi P(f)) \overline{R_{q_{2}}}(\varphi
\end{gathered}
$$


For revamp process,

$$
\begin{gathered}
L_{q}(f)=\frac{L_{q}(0, f)(1-L(\varphi-\varphi P(f)+\omega))}{\varphi-\varphi P(f)+\omega} \\
\int_{0}^{\infty} L_{q}(x, f) \varepsilon(x) d x=\frac{\omega f\left(1-\overline{Q_{b}}(\varphi-\varphi P(f)+\omega)\right)\left(\bar{L}(\varphi-\varphi P(f)+\omega) S_{q}^{(a)}(0, f) \overline{Q_{a}}(\varphi-\varphi P(f))\right)}{(\varphi-\varphi P(f)+\omega)}
\end{gathered}
$$

Using (32), (34), (36) in (21), we get:

$$
S_{q}^{(a)}(0, f)=-\frac{D(\varphi-\varphi P(f))}{f-\overline{Q_{a}}(\varphi-\varphi P(f))\left\{\frac{1-\bar{Q}(\varphi-\varphi P(f)+\omega)}{\varphi-\varphi P(f)+\omega}\right\} L(\varphi-\varphi P(f)+\omega) \omega f-\overline{Q_{a}}(\varphi-\varphi P(f)) \overline{Q_{b}}(\varphi-\varphi P(f)+\omega) \overline{R_{q_{1}}}(\varphi-\varphi P(f))\left(c \overline{R_{q_{2}}}(\varphi\right.}
$$

Substituting (37) in (27),(29),(31),(33) we get:

$$
\begin{gathered}
S_{q}^{(a)}(f)=\frac{S_{q}^{(a)}(0, f)\left(1-\overline{Q_{a}}(\varphi-\varphi P(f))\right)}{\varphi-\varphi P(f)} \\
S_{q}^{(b)}(f)=\frac{S_{q}^{(b)}(0, f)\left(1-\overline{Q_{b}}(\varphi-\varphi P(f)+\omega)\right)}{\varphi-\varphi P(f)+\omega} \\
B_{q}^{(1)}(f)=\frac{B_{q}^{(1)}(0, f)\left(1-R_{q_{1}}(\varphi-\varphi P(f))\right)}{\varphi-\varphi P(f)} \\
B_{q}^{(2)}(f)=\frac{B_{q}^{(2)}(0, f)\left(1-R_{q_{2}}\left(\varphi-\varphi P(f)+\tau-\frac{\tau}{f}\right)\right)}{\varphi-\varphi P(f)+\tau-\frac{\tau}{f}} \\
L_{q}(f)=\frac{L_{q}(0, f)(1-L(\varphi-\varphi P(f)+\omega))}{\varphi-\varphi P(f)+\omega}
\end{gathered}
$$

\section{PROBABILITY GENERATING FUNCTION OF THE QUEUE LENGTH}

Let $A^{* *}(f)$ be the likelihood producing capacity of the line length regardless of what the framework is,

$$
\text { (i.e.) } A^{* *}(f)=S_{q}^{(a)}(f)+S_{q}^{(b)}(f)+B_{q}^{(1)}(f)+B_{q}^{(2)}(f)+L_{q}(f)
$$

Thus adding (26),(29),(31),(33) and (35) we get:

$$
A^{* *}(z)=\frac{D\left\{\left(-\left(1-\overline{Q_{a}}(\varphi-\varphi P(f))\right)-\left(1-\overline{R_{q_{1}}}(\varphi-\varphi P(f))\right) \overline{Q_{a}}(\varphi-\varphi P(f)) \overline{Q_{b}}(\varphi-\varphi P(f)+\omega)+\omega f \overline{Q_{a}}(\varphi-\varphi P(f))\left\{\frac{1-Q_{b}(\varphi-\varphi P(f)+\omega)}{\varphi-\varphi P(f)+\omega}\right\} \bar{L}\right.\right.}{D(z)}
$$


Where:

$D(z)=f-\overline{Q_{a}}(\varphi-\varphi P(f))\left\{\frac{1-Q_{b}(\varphi-\varphi P(f)+\omega)}{\varphi-\varphi P(f)+\omega}\right\} \bar{L}(\varphi-\varphi P(f)+\omega) \omega f-\overline{Q_{a}}(\varphi-\varphi P(f)) \overline{Q_{b}}(\varphi-\varphi P(f)+\omega)$

\section{IDLE TIME AND UTILIZATION FACTOR}

The normalization condition $A^{* *}(1)+D=1 \quad(44)$.

Gives out the idle time and hence the time spent by the server for the service.

L'Hopital's rule is applied on (43) due to its determinant form to achieve

$$
\lim _{z \rightarrow 1} A^{* *}(z)=\frac{\varphi\left[1+E\left(Q_{a}\right)+E\left(R_{q_{1}}\right)\right]+\left(1-Q_{b}(\omega)\right) E(L)}{1-E\left(Q_{a}\right) \varphi\left(1-\overline{Q_{b}}(\omega)\right)-E\left(Q_{b}\right) \omega-E(L) \varphi\left(1-\overline{Q_{b}}(\omega)\right)-\left(1-\overline{Q_{b}}(\omega)\right)+\varphi\left[E\left(Q_{a}\right)+E\left(Q_{b}\right)+E\left(R_{q_{1}}\right)\right]-(\tau-\varphi) c E\left(R_{q_{2}}\right)}
$$

Now using (45) and normalizing condition we get

$$
D=\frac{1-E\left(Q_{a}\right) \varphi\left(1-\overline{Q_{b}}(\omega)\right)-E\left(Q_{b}\right) \omega-E(L) \varphi\left(1-\overline{Q_{b}}(\omega)\right)-\left(1-\overline{Q_{b}}(\omega)\right)+\varphi\left[E\left(Q_{a}\right)+E\left(Q_{b}\right)+E\left(R_{q}\right)\right]-(\tau-\varphi) c E}{\left[\varphi\left[1+E\left(Q_{a}\right)+E\left(R_{q}\right)\right]+\left(1-\overline{Q_{b}}(\omega)\right) E(L)\right]\left\{1-E\left(Q_{a}\right) \varphi\left(1-\overline{Q_{b}}(\omega)\right)-E\left(Q_{b}\right) \omega-E(L) \varphi\left(1-\overline{Q_{b}}(\omega)\right)-\left(1-\overline{Q_{b}}(\omega)\right)+\varphi\left[E\left(Q_{a}\right)+E(Q\right.\right.}
$$

Also from the above, the utilization factor can be calculated, using $\rho=1-D$

\section{EXECUTION MEASURES OF THE LINING SYSTEM}

To find $L_{q}$, where $L_{q}=\frac{d}{d z} A^{* *}(z) \mid z=1$

As it takes indeterminate form as z tends to 1, L 'Hopital's rule is applied. Hence

$$
\begin{gathered}
L_{q}=\lim _{z \rightarrow 1} \frac{D^{\prime}(z) N^{\prime \prime}(z)-N^{\prime}(z) D^{\prime \prime}(z)}{2\left(D^{\prime}(z)\right)^{2}} \\
D^{\prime}(1)=1-E\left(Q_{a}\right) \varphi\left(1-\overline{Q_{b}}(\omega)\right)-E\left(Q_{b}\right) \omega-E(L) \varphi\left(1-\overline{Q_{b}}(\omega)\right)-\left(1-\overline{Q_{b}}(\omega)\right)+\varphi \\
D^{\prime \prime}(1)=-2 \varphi E\left(Q_{a}\right) E\left(Q_{b}\right) \omega-3 \varphi\left(1-\overline{Q_{b}}(\omega)\right) E(L)-2 \varphi E\left(Q_{a}\right)\left(1-\overline{Q_{b}}(\omega)\right)-E\left(Q_{b}^{2}\right) \varphi \\
N^{\prime}(1)=\varphi\left[1+E\left(Q_{a}\right)+E\left(R_{q_{1}}\right)\right]+\left(1-\overline{Q_{b}}(\omega)\right) E(L) \\
N^{\prime \prime}(1)=\varphi^{2}\left[E\left(Q_{a}^{2}\right)+\overline{Q_{b}}(\omega)\left(E\left(R_{q_{1}}^{2}\right)+2 E\left(R_{q_{1}}\right) E\left(Q_{a}\right)+\frac{c}{\varphi}\right)+2 E\left(R_{q_{1}}\right) E\left(Q_{b}\right)\right]-\varphi E
\end{gathered}
$$


The above derivatives are substituted in (47) we obtain $L_{q}$ in closed form. Further the other performance measures of the defined queuing model are found using Little's formula $W_{q}=\frac{L_{q}}{\lambda} \quad,=\frac{L}{\lambda}, L=L_{q}+\rho$.

\section{NUMERICAL ILLUSTRATION}

We portray a numerical point of reference with the ultimate objective to see the effect and authenticity of our results of the particular parameters used in our model, to be explicit reneging rate, separate rate and likelihood of culmination of fix rate on the utilization factor and execution extents of the coating model. The estimations of the parameters are assembled with the ultimate objective that the unfaltering condition isn't harmed.

Assume service time follows an exponential distribution:

$$
\begin{aligned}
& E\left(Q_{a}\right)=\frac{1}{\xi_{a}}, E\left(Q_{a}^{2}\right)=\frac{2}{\xi_{a}^{2}}, E(L)=\frac{1}{\varepsilon}, \overline{Q_{b}}(\omega)=\frac{\xi_{b}}{\xi_{b}+\omega}, E\left(L^{2}\right)=\frac{2}{\varepsilon^{2}}, \\
& {\overline{Q_{b}}}^{\prime}(\omega)=\frac{-\xi_{b}}{\left(\xi_{b}+\omega\right)^{2}},{\overline{Q_{b}}}^{\prime \prime}(\omega)=\frac{2 \xi_{b}}{\left(\xi_{b}+\omega\right)^{3}}, E\left(R_{q_{1}}\right)=\frac{1}{\gamma}, E\left(R_{q_{2}}\right)=\frac{1}{\delta}, \\
& E\left(R_{q_{1}}^{2}\right)=\frac{2}{\gamma^{2}}, E\left(R_{q_{2}}^{2}\right)=\frac{2}{\delta^{2}}, \xi_{a}=4, \xi_{b}=5, \omega=2, \gamma=3.5, \delta=2.5 \\
& , \epsilon=5, \tau=3, \varphi=3, c=0.5
\end{aligned}
$$

Case (I): Table 1 demonstrate that for steady estimations of all the parameters and expanding the arrival rate $\varphi$, demonstrates an expansion in all the executions estimates like $L_{q^{\prime}} L, W_{q^{\prime}}$ $W$. Additionally, it prompts a contraction in usage factor and at the same time an increase in idle time.

Table 1. Effect of Variation Of Arrival Rate $\varphi=1,2.5,3,4,5,6,7$.

\begin{tabular}{|c|c|c|c|c|c|}
\hline $\boldsymbol{Q}$ & $\boldsymbol{\rho}$ & $\boldsymbol{L}_{\boldsymbol{q}}$ & $\boldsymbol{W}_{\boldsymbol{Q}}$ & $\boldsymbol{L}$ & $\boldsymbol{W}$ \\
\hline 0.3623 & 0.6377 & 0.5463 & 0.1821 & 1.184 & 0.3947 \\
\hline 0.3782 & 0.6218 & 0.8571 & 0.2143 & 1.4789 & 0.3697 \\
\hline 0.3875 & 0.6125 & 1.0928 & 0.2186 & 1.7053 & 0.3411 \\
\hline 0.3935 & 0.6065 & 1.2994 & 0.2166 & 1.9059 & 0.3177 \\
\hline 0.4071 & 0.5929 & 1.4398 & 0.2057 & 2.0327 & 0.2904 \\
\hline
\end{tabular}




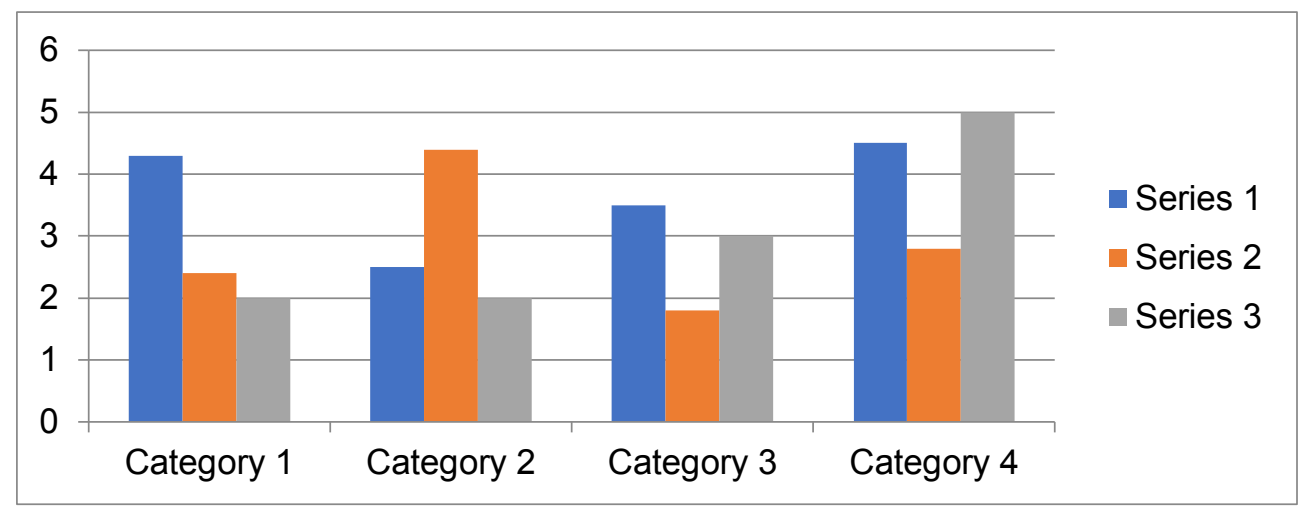

Graphic 1. Variation of $\varphi$.

Case (II): From Table 2, It is clear that if the probability in completion of repair rate increases, it leads to a decrease in all the performance measures. Since the repair gets completed sooner, the idle time of the server increases, and the time spent by the server for service get expanded.

Table 2. Effect of Variation of Repair Rate $\epsilon=5,6,7,8,9$.

\begin{tabular}{|c|c|c|c|c|c|}
\hline $\boldsymbol{Q}$ & $\boldsymbol{\rho}$ & $\boldsymbol{L}_{\boldsymbol{q}}$ & $\boldsymbol{W}_{\boldsymbol{Q}}$ & $\boldsymbol{L}$ & $\boldsymbol{W}$ \\
\hline 0.2822 & 0.7178 & 0.2080 & 0.0693 & 0.9258 & 0.3086 \\
\hline 0.2857 & 0.7143 & 0.1610 & 0.0537 & 0.8753 & 0.2918 \\
\hline 0.2883 & 0.7117 & 0.1310 & 0.0437 & 0.8427 & 0.2809 \\
\hline 0.2902 & 0.7098 & 0.1105 & 0.0368 & 0.8203 & 0.2734 \\
\hline 0.2916 & 0.7084 & 0.0956 & 0.0319 & 0.8040 & 0.2680 \\
\hline
\end{tabular}

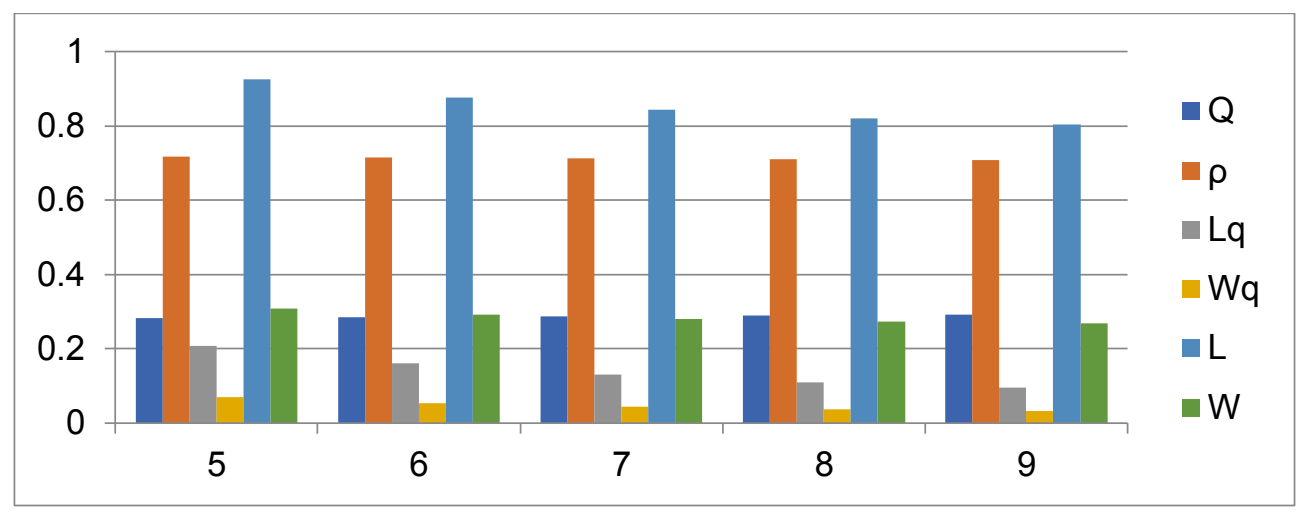

Graphic 2. Variation of $\varepsilon$. 
Case (III): Table 3 indicates that an increase in Reneging rate $\tau$ makes all the performance measures to decrease. Additionally, it prompts an expansion in usage factor and at the same time a decrease out of idle time.

Table 3. Effect of Variation of Standby Server Rate $\tau=3,3.5,4,4.5,5,5.5$.

\begin{tabular}{|c|c|c|c|c|c|}
\hline $\boldsymbol{Q}$ & $\boldsymbol{\rho}$ & $\boldsymbol{L}_{\boldsymbol{q}}$ & $\boldsymbol{W}_{\boldsymbol{Q}}$ & $\boldsymbol{L}$ & $\boldsymbol{W}$ \\
\hline 0.3709 & 0.6291 & 0.5819 & 0.1940 & 1.2110 & 0.4037 \\
\hline 0.3623 & 0.6377 & 0.5461 & 0.1820 & 1.1838 & 0.3946 \\
\hline 0.3535 & 0.6465 & 0.4822 & 0.1607 & 1.1287 & 0.3762 \\
\hline 0.3444 & 0.6556 & 0.3833 & 0.1278 & 1.0389 & 0.3463 \\
\hline 0.3350 & 0.6650 & 0.2417 & 0.0806 & 0.9067 & 0.3022 \\
\hline 0.3254 & 0.6746 & 0.0465 & 0.0155 & 0.7211 & 0.2404 \\
\hline
\end{tabular}

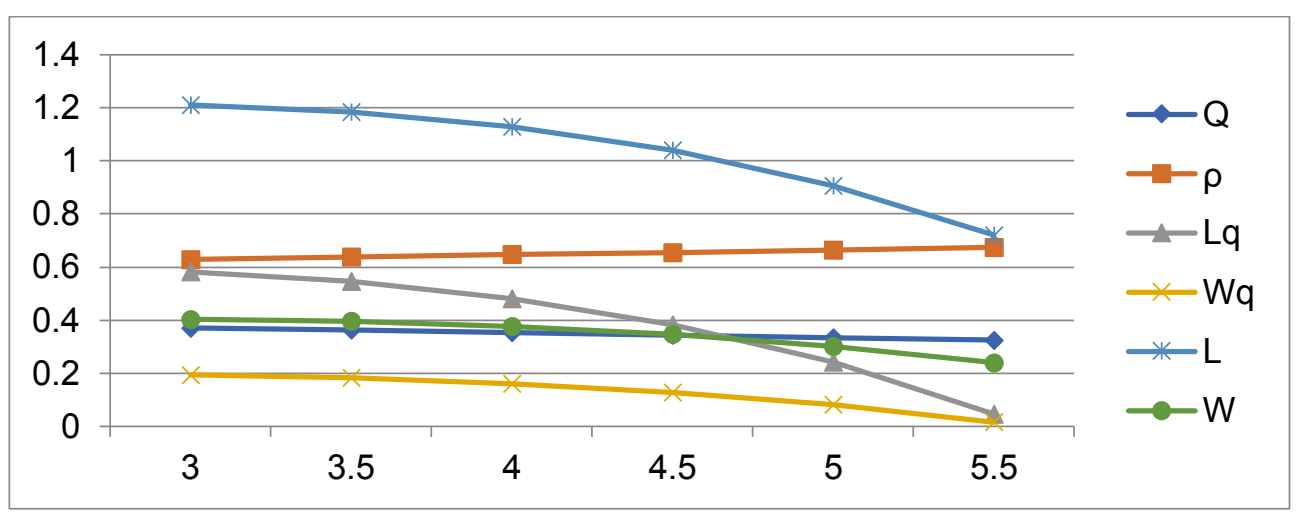

Graphic 3. Variation of $\tau$.

Case (IV): Table 4 demonstrates that for consistent estimations of all the parameters and expanding the completion of the compulsory vacation rate $\gamma$, demonstrates a contraction in all the executions estimates like $L_{q}, L, W_{q}, W$. Furthermore it prompts a development in use factor and in the meantime a decline out of inert time.

Table 4. Effect of Variation of Vacation rate $\gamma=2.5,3,4,5,6$.

\begin{tabular}{|c|c|c|c|c|c|}
\hline $\boldsymbol{Q}$ & $\boldsymbol{\rho}$ & $\boldsymbol{L}_{\boldsymbol{q}}$ & $\boldsymbol{W}_{\boldsymbol{Q}}$ & $\boldsymbol{L}$ & $\boldsymbol{W}$ \\
\hline 0.3030 & 0.697 & 0.8895 & 1.5865 & 1.7965 & 0.5288 \\
\hline 0.2914 & 0.7086 & 0.7885 & 0.2628 & 1.4971 & 0.499 \\
\hline 0.2748 & 0.7252 & 0.5499 & 0.1833 & 1.2751 & 0.425 \\
\hline 0.2635 & 0.7365 & 0.3046 & 0.1015 & 1.0411 & 0.347 \\
\hline 0.2553 & 0.7447 & 0.0713 & 0.0238 & 0.8160 & 0.2720 \\
\hline
\end{tabular}




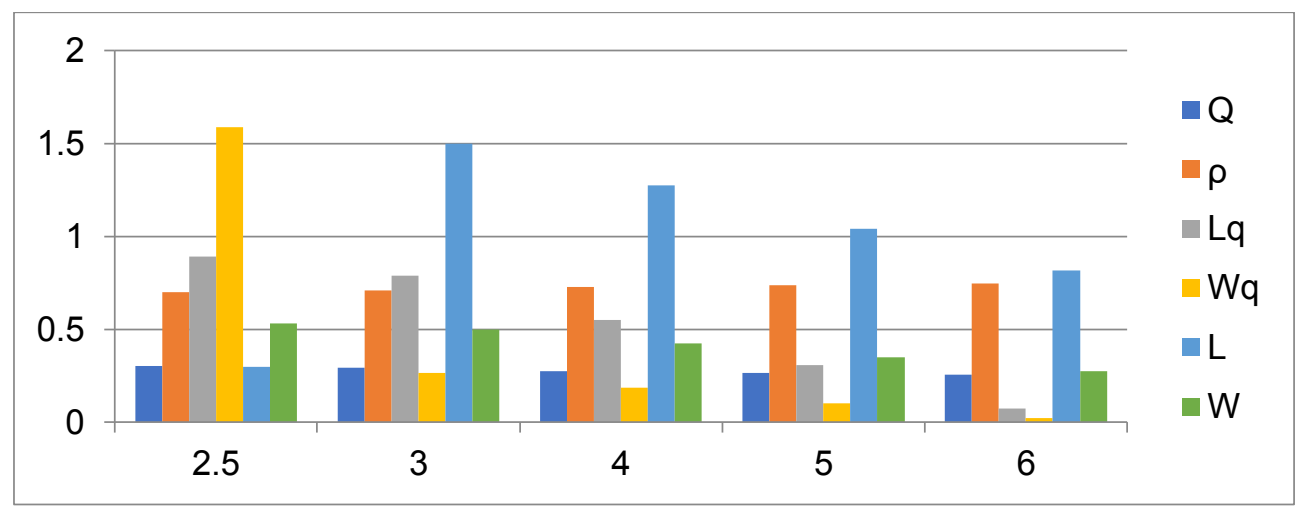

Graphic 4. Variation of $\gamma$.

\section{CONCLUSION}

The model portrayed finds its applications in various divisions of amassing organizations and correspondence frameworks. It is extraordinarily sensible to depict about stay by the server in the midst of optional extended vacation. This kind of vacation makes the server complete their help, work rely on need. As it isn't deterministic, if the upkeep work is most outrageous it can take a long journey or else short escape time. Graphical depiction pictures the model broadly and gives the reasonable results as obvious. As a future work, shying without end can be displayed. Need standard can be included. Likewise the re-try strategy can be given in stages subject to the kind of fix. Postpone process can moreover be considered before getting into a fix up process. Set up time criteria can be included in this concept of Queuing. This presentation accepts prominent employment in gathering units, correspondence structure, and movement crossing focuses, system designing and so on. 


\section{REFERENCES}

Altman, E., \& Yechiali, U. (2006). Analysis of customer's impatience in queue with server vacations. Queueing systems, 5(4), 261-279. https://doi.org/10.1007/s11134006-6134-x

Artalejo, R. J., \& Choudhury, G. (2004). Steady state analysis of an M/G/1 queue with repeated attempts and two phase service. Quality Technology and Quantitative Management,1(2), 189-199. https://doi.org/10.1080/16843703.2004.11673072

Doshi, B. T. (1986). Queueing systems with vacations-a survey. Queueing systems, 1(12), 2966. https://doi.org/10.1007/BF01149327

Keilson, J., \& Servi, L. D. (1987). The dynamics of an M/G/1 vacation model. Operations research, 35(4), 575-582. https://doi.org/10.1287/opre.35.4.575

Lee, H. W., Lee, S. S., \& Chae, K. G. (1994). Operating characteristics of M[X]/G/1 queue with N policy. Queueing system, 15(1-4), 387-399. https://doi.org/10.1007/ BF01189247

Madan, K. G. (2000). On a single server queue with two stage heterogeneous service ad deterministic server vacations. The Egyptian Statistical journal, 44(1), 39-55.

Madan, K. G., \& Abu Al-Rub, A. Z. (2004). On a single server queue with optional phase type server vacations based on exhaustive deterministic service and a single vacation policy. Applied Mathematics and Computation, 149, 723-734. https://doi.org/10.1016/ S0096-3003(03)00174-7

Madan, K. C., \& Baklizi, A. (2002). An M/G/1 queue with additional second stage service and optional re service. International journal of information and Management sciences, 16(2), 1-16. https://www.researchgate.net/publication/285743897_An_MG1_ queue_with_additional_second_stage_service_and_optical_re-service 
Maragathasundari, S., \& Balamurugan, B. (2015). A study on the performance analysis of a batch arrival queue with two stages of service Bernoulli schedule vacation extended vacation and service interruption. International journal of computer applications, 124(12), 33-37. https://www.ijcaonline.org/archives/volume124/ number12/22159-2015905695

Maragathasundari, S., \& Dhanalakshmi, K. S. (2018). Mobile adhoc networks problem- A queuing approach. International journal of communication networks and distributed systems, 21(4). https://doi.org/10.1504/IJCNDS.2018.095363

Maragathasundari, S., \& Srinivasan, S. (2012). Analysis of M/G/1 feedback queue with three stages and multiple server vacation. Applied mathematical sciences, 6(125), 6221-6240.

Maragathasundari, S., \& Srinivasan, S. (2015). A Non-Markovian Multistage Batch arrival queue with breakdown and reneging. Mathematical problems in engineering. Article ID 519579. http://dx.doi.org/10.1155/2014/519579

Maragathasundari, S., \& Srinivasan, S. (2017). Optional services in a Non Markovian Queue. International fournal of Operational Research, 28(1), 1-17. https://doi. org/10.1504/IJOR.2017.10000660

Maraghi, F. A., Madan, K. C., \& Darby-Dowman, K. (2010). Batch Arrival vacation queue with second optional service and Random Breakdowns. International Fournal of Statistical Theory and Practice, 4(1), 137-153. https://doi.org/10.1080/15598608.2010 .10411977 


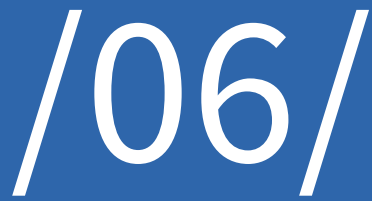




\title{
DESIGN AND OPTIMIZATION OF REVERSIBLE LOOK AHEAD CARRY ADDER AND CARRY SAVE ADDER
}

\author{
N. Bhuvaneswary \\ Assistant Professor. Department of ECE. \\ Kalasalingam Academy of Research and Education, (India). \\ E-mail: bhuvaneswary.n@klu.ac.in ORCID: http://orcid.org/0000-0001-6400-6602 \\ A. Lakshmi \\ Associate Professor. Department of ECE. \\ Kalasalingam Academy of Research and Education, (India). \\ E-mail: lakshmi@klu.ac.in ORCID: http://orcid.org/0000-0002-6744-7048
}

Recepción: 05/12/2019 Aceptación: 03/01/2020 Publicación: 23/03/2020

\section{Gitación sugerida:}

Bhuvaneswar, N., y Lakshmi, A. (2020). Design and optimization of reversible look ahead carry adder and carry save adder. 3C Tecnología. Glosas de innovación aplicadas a la pyme. Edición Especial, Marzo 2020, 113-127. http://doi.org/10.17993/3ctecno.2020.specialissue4.113-127

\section{Suggested citation:}

Bhuvaneswar, N., \& Lakshmi, A. (2020). Design and optimization of reversible look ahead carry adder and carry save adder. 3C Tecnología. Glosas de innovación aplicadas a la pyme. Edición Especial, Marzo 2020, 113-127. http://doi.org/10.17993/3ctecno.2020.specialissue4.113-127 


\section{ABSTRACT}

A circuit is said to be reversible if inputs and the outputs are equal. Reversibility focused mainly to bring down power to zero. In modern centuries, gates with reversible logic has arose together as notable vital approaches for power optimisation based on application. Reversible logic is leading area in power consumption. Based on its application, its emerging trend in power consumption. In ideal situations, reversible circuit yield nil power. In this paper, new design of the look ahead carry adder and carry save adder designed and it is optimized with the previous existing binary logic gates. Minimizing the garbage output and replacing the binary logic gates by reversible logic gates. To develop low power circuits, reversible circuit is necessary.

\section{KEYWORDS}

Look ahead carry adder, Carry save adder, Reversible logic. 


\section{INTRODUCTION}

Look ahead carry adder is a type of digital adder. In this circuit, speed can be increased by reducing the required time. Generated carry bit calculated before summing so that it can reduce the time delay. The design of ripple carry circuit is modest, but it has timeconsuming delay in the circuit due to several gates in path carry flows from LSB to MSB. Therefore, in this paper designed an alternate design, look ahead carry adder. For designing look ahead carry adder, transform ripple carry design to strategy, which reduce the number of bits to two level bit logic.

By using carry-save adder design sum up multiple binary numbers. When compared to other adder design, carry look ahead adder design be at variance in dualistic outputs that has same aspect as inputs, first output has been series of half done sum and next output has been series of carry.

\section{A. NEED FOR REVERSIBLE LOGIC}

Reversible circuits are effectual than irreversible because of information loss which leads to energy loss. Due to information loss in irreversibility, it dissipates more power. To reduce power, circuit designed with reversible logic. At last, reversible circuits can be viewed as distinct instance of quantum circuits since quantum progression must be reversible.

\section{B. CONDITIONS FOR REVERSIBLE COMPUTATION}

Reversible computation satisfies the conditions.

\section{The foremost State:}

Formost state is logical reversibility in which any settled device to be reversible state and the input and output should be unambiguously recoverable from one another.

\section{The second State:}

The second state is physical reversibility, the device in reality run backwards, i.e., each operation converts no energy to heat and produces no entropy. 


\section{Representation of a reversible circuit truth table}

In view of the fact that we are dealing only with bijective functions, i.e., permutations, we signify them using the pedal system which represented by dislodge cycle of functions.

$S_{n}$, denoted as set of all permutations of $n$ indices and $S_{2}^{n}$ mentioned as set of objective perform with $n$ input binary bits. Let us Toffoli's Gate and its corresponding truth table.

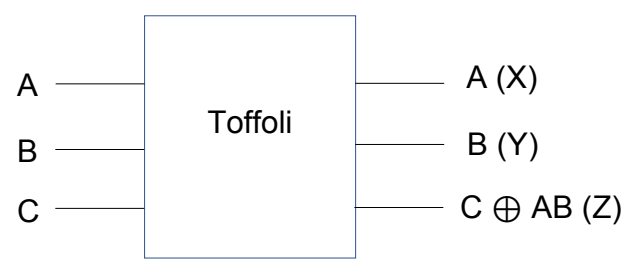

Figure 1. Toffoli gate.

Table 1. Truth table for toffoli gate.

\begin{tabular}{|l|l|l|l|l|l|}
\hline \multicolumn{3}{|c|}{ Inputs } & \multicolumn{3}{c|}{ Outputs } \\
\hline A & B & C & X & $y$ & Z \\
\hline $\mathbf{0}$ & 0 & 0 & 0 & 0 & 0 \\
\hline $\mathbf{0}$ & 0 & 1 & 0 & 0 & 1 \\
\hline $\mathbf{0}$ & 1 & 0 & 0 & 1 & 0 \\
\hline $\mathbf{0}$ & 1 & 1 & 0 & 1 & 1 \\
\hline $\mathbf{1}$ & 0 & 0 & 1 & 0 & 0 \\
\hline $\mathbf{1}$ & 0 & 1 & 1 & 0 & 1 \\
\hline $\mathbf{1}$ & 1 & 0 & 1 & 1 & 1 \\
\hline $\mathbf{1}$ & 1 & 1 & 1 & 1 & 0 \\
\hline
\end{tabular}

\section{Some special types of Reversible Gates}

\section{SWAP Gate:}

Reversible gate, called the SWAP (S) gate which interchanges the input.

\section{Toffoli's Gate:}

In Toffoli Gate (Agarwal, Choudhary, Jangid, \& Kasera, 2017), all the inputs that is from 1 to (n-1) are mapped to its corresponding outputs. The final output is coordinated by inputs from 1 to (n-1). To upended and pass the nth input make all inputs as 1 else pass original 
output. The first two inputs corresponds to outputs and the third output controlled by first two input and invert it. The truth table has been shown in Table.

\section{MTSG gate:}

In MTSG gate (Agarwal et al., 2017) four number of inputs and outputs are used. By this design the one bit full adder is designed.

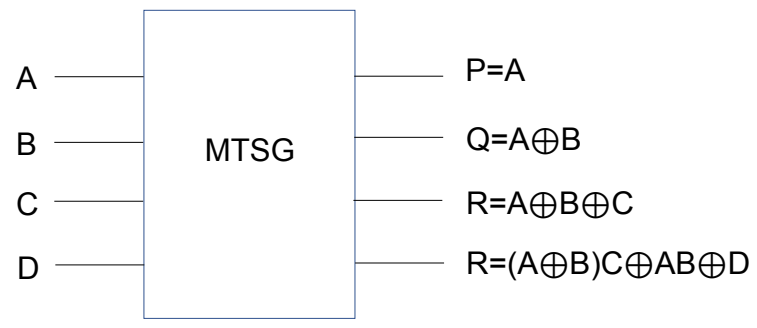

Figure 2. MTSG gate.

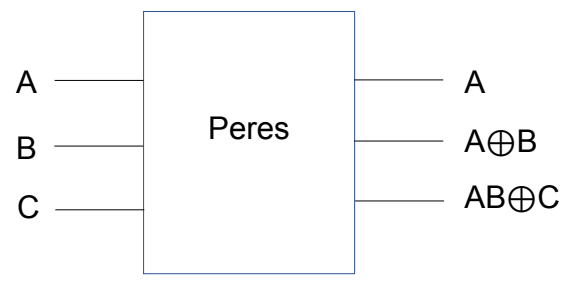

Figure 3. Peres gate.

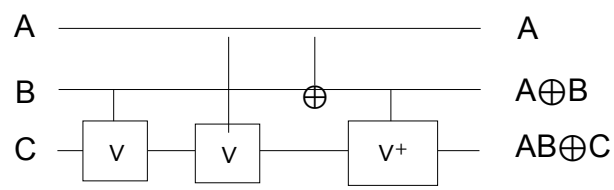

Figure 4. Internal architecture with Peres gate.

Table 2. Peres gate truth table.

\begin{tabular}{|c|c|c|c|c|c|}
\hline $\mathbf{A}$ & $\mathbf{B}$ & $\mathbf{C}$ & $\mathbf{P}$ & $\mathbf{Q}$ & $\mathbf{R}$ \\
\hline 0 & 0 & 0 & 0 & 0 & 0 \\
\hline 0 & 0 & 1 & 0 & 0 & 1 \\
\hline 0 & 1 & 0 & 0 & 1 & 0 \\
\hline 0 & 1 & 1 & 0 & 1 & 1 \\
\hline 1 & 0 & 0 & 1 & 1 & 0 \\
\hline 1 & 0 & 1 & 1 & 1 & 1 \\
\hline 1 & 1 & 0 & 1 & 0 & 1 \\
\hline 1 & 1 & 1 & 1 & 0 & 0 \\
\hline
\end{tabular}




\section{LOOK AHEAD CARRY ADDER}

\section{A. CONGEPT OF GARRY GENERATOR}

Look ahead carry adder used to produce and circulate carry. In binary addition when both the inputs are 1, it generates carry and propagate

If either of the input is 1 then $\mathrm{A}+\mathrm{B}$ propagates in case binary addition. Binary predicate is represented as $P(A, B)$

$P(A, B)=A+B$

If binary addition, then expression can be represented as:

$P^{\prime}(A, B)=A$ xor $B$

Binary operation performs faster than xor. Though we can use P'(A, B) for multiple bit carry look ahead adder.

In Boolean function, $P_{i}$ represented as propagate, $\mathrm{Ci}$ denoted as carry bit and $G_{i}$ generate binary bit.

$\mathrm{Ci}+\mathrm{l}=\mathrm{Gi}+(\mathrm{Pi}$.Ci $)$

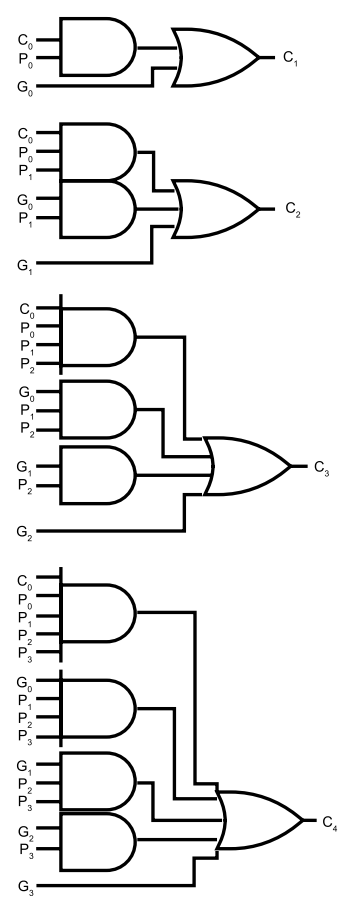

Figure 5. Existing model of carry generator. 


\section{PROPOSED ADDER ARCHITECTURES}

This deals with the Design and operation of the proposed look ahead carry adder architecture using Peres gate based on the existing adder architecture. The proposed architectures are implemented by replacing the three block's (Peres full adder) Peres gates and peresfull adders with reversible logic gates to obtain the better performance compared to conventional logic.

\section{Design I}

In this sector look ahead carry adder using Peres logic is proposed. As we know the Peres logic already, it is pretty much easier to propose this type of adder using the Peres reversible gate. The Peres full adder is already proposed (Somani, Chaudhary, \& Yadav, 2016; Lisa, $\&$ Babu, 2015).

Whenever the quantum cost of the Peres gate is said to be four and the Peres full adder consist of two Peres gate, which proposes the quantum cost of eight. In addition, the minimal number of reversible logic gates used for proposing a 4 bit look ahead carry adder is 32. This design proposes the 4 bit look ahead carry adder design consist of four sum elements and a carry output.

\section{PROPOSED LOOK AHEAD GARRY ADDER}

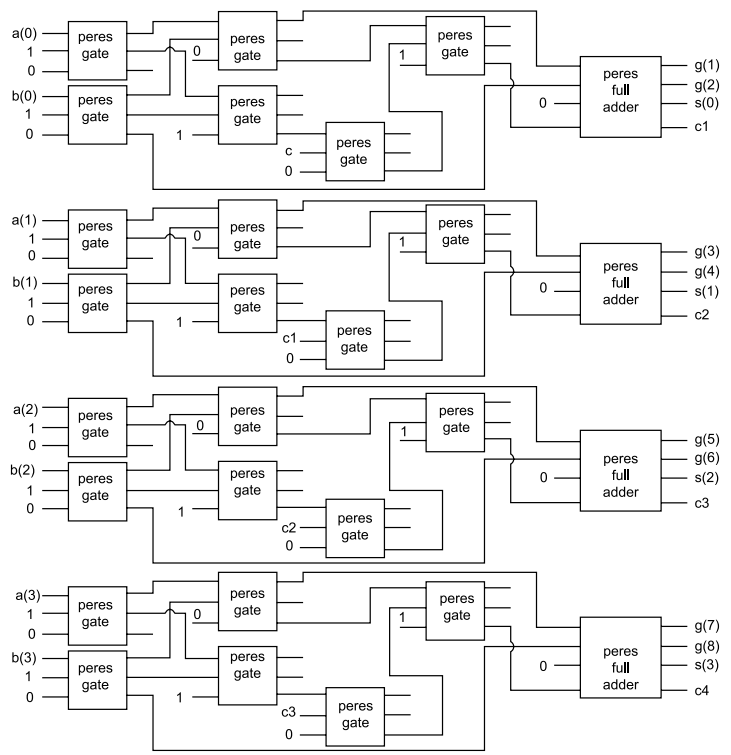

Figure 6. Look ahead carry adder Design 1. 


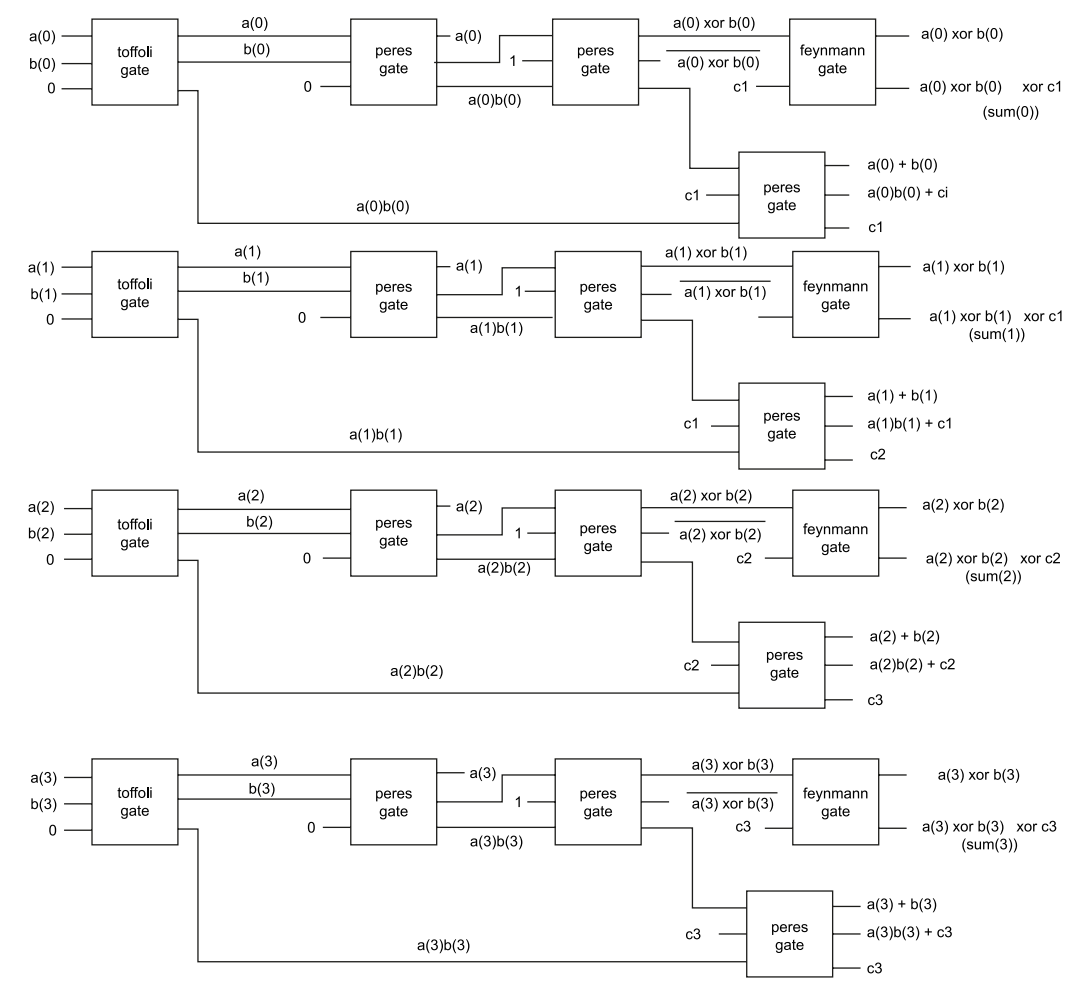

Figure 7. Look ahead carry adder Design 2.

This second design is proposed by using three types of reversible gates (Peres, Toffoli, Feynmann) (Somani et al., 2016) although it is already proposed through the survey that the gates quantum cost (Peres, Toffoli, Feynmann) (Somani et al., 2016) are 4,5 and 1 respectively. By proposing this adder the quantum cost and the count of garbage outputs are also reduced. The above design proposes a design with four sum elements and a single carry output. And this design has a quantum cost of 18 for a single bit adder.

\section{B. PROPOSED GARRY SAVE ADDER}

This deals with the designing and optimization of the carry save adder by replacing the conventional logic gates by the reversible gates. By considering the minimal quantum cost containing design as the best design. 


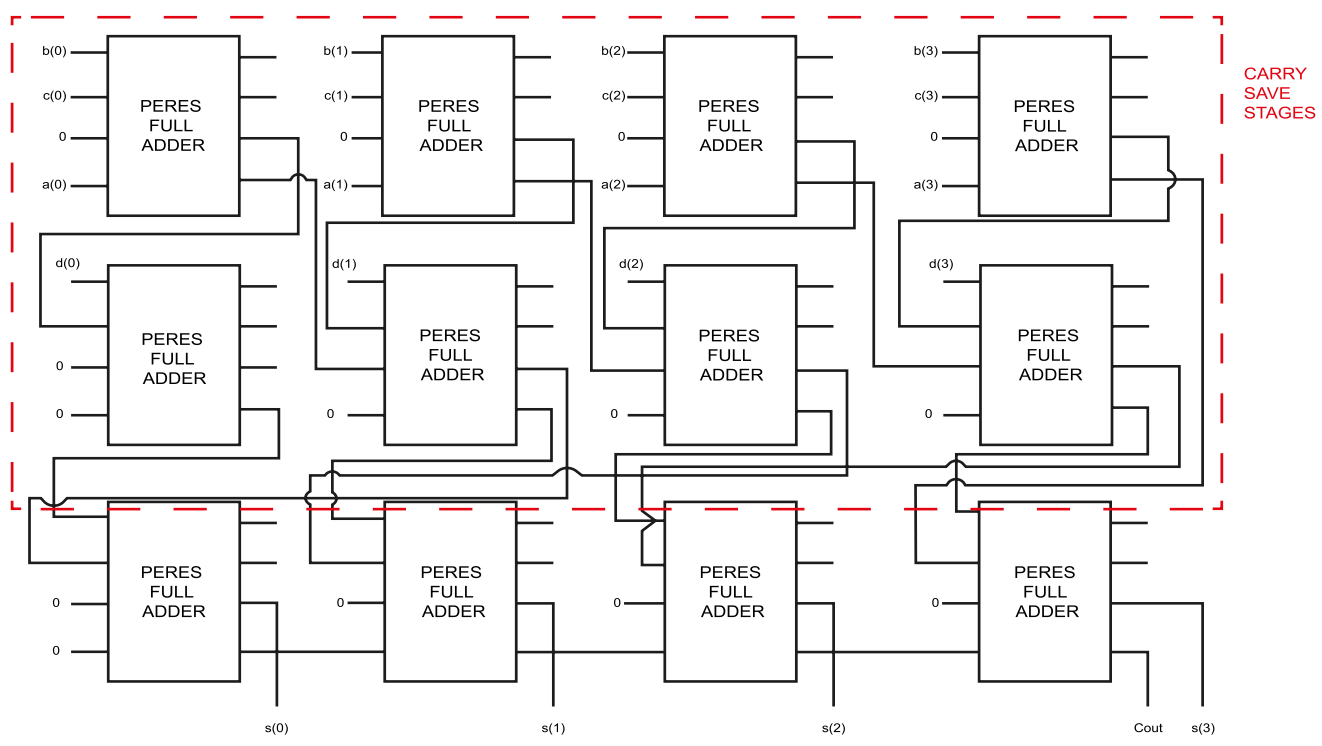

Figure 8. Design 1.

Proposed 4 bit carry save adder is designed Peres full adder. Source: (Somani et al., 2016).

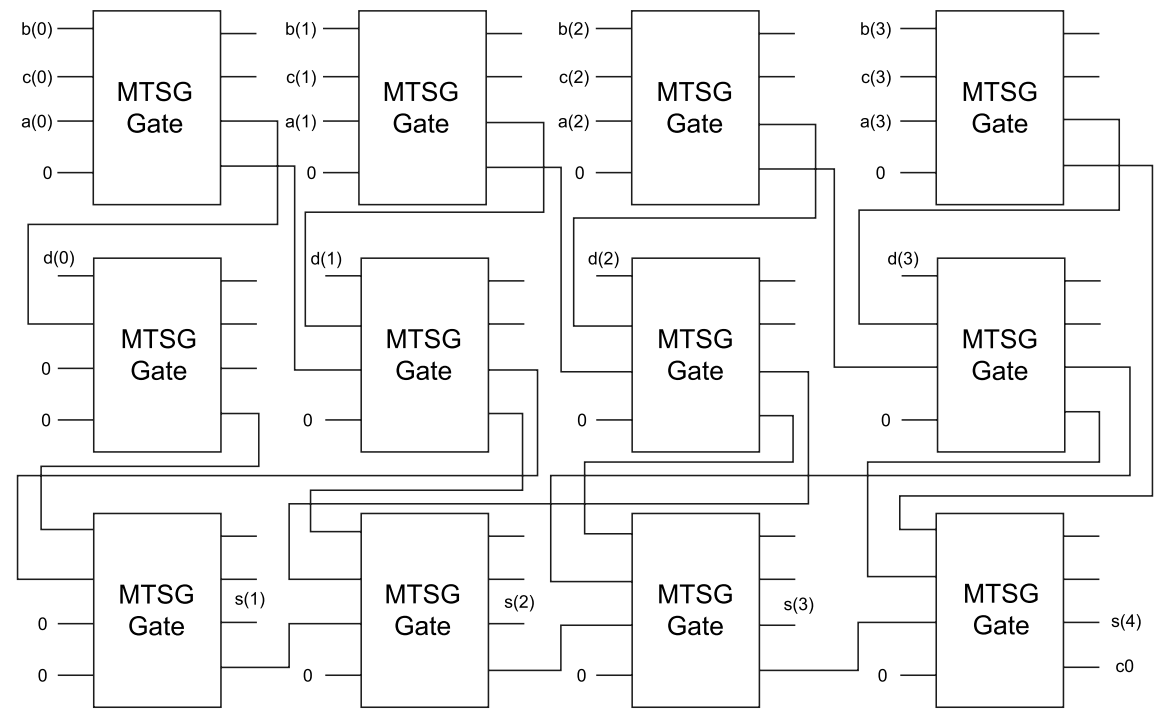

Figure 9. Design 2.

This design proposed by using the MTSG gates.

\section{SIMULATION RESULTS}

Proposed reversible adder circuit is more proficient than existing method. Based on the comparative analysis, proposed design can be easily realized. In existing design, logic gate 
used instead of that in proposed work reversible gate such as Peres used to understand the circuit. The circuit realized using reversible Peres gate to reduce the logical calculation. Also in terms of hardware complexity proposed work is efficient than exiting circuit.

All the simulations have been done using XILINX 9.2i and Model sim Altera 6.3g_p1.

\section{SIMULATION RESULTS OF PROPOSED ARGHITECTURE}

Design I

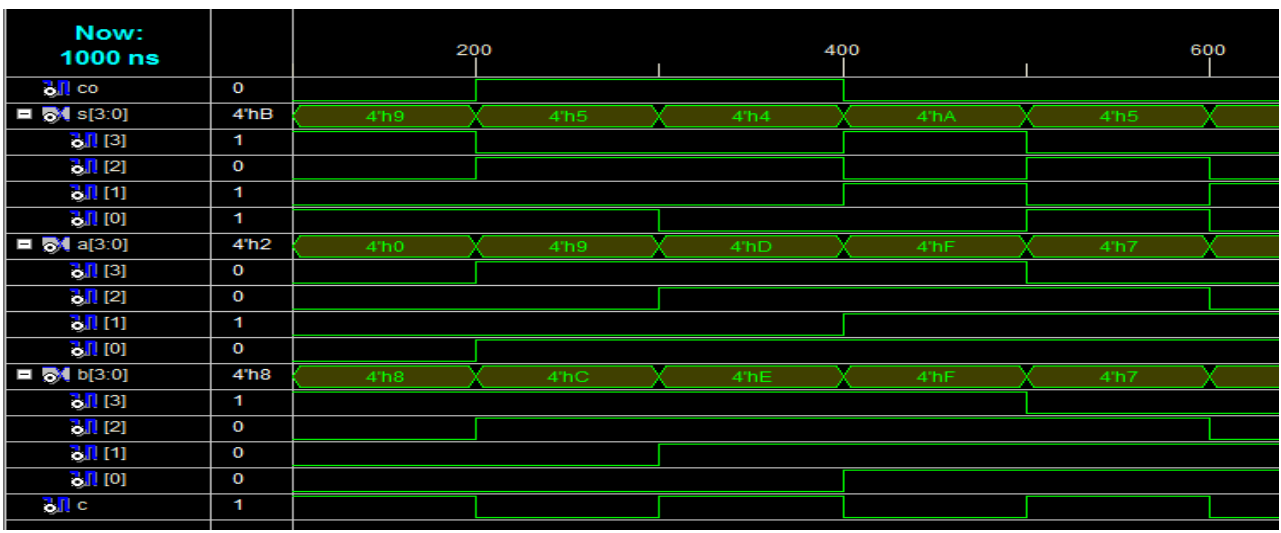

Figure 10. Proposed Design 1 Look ahead carry adder simulation results.

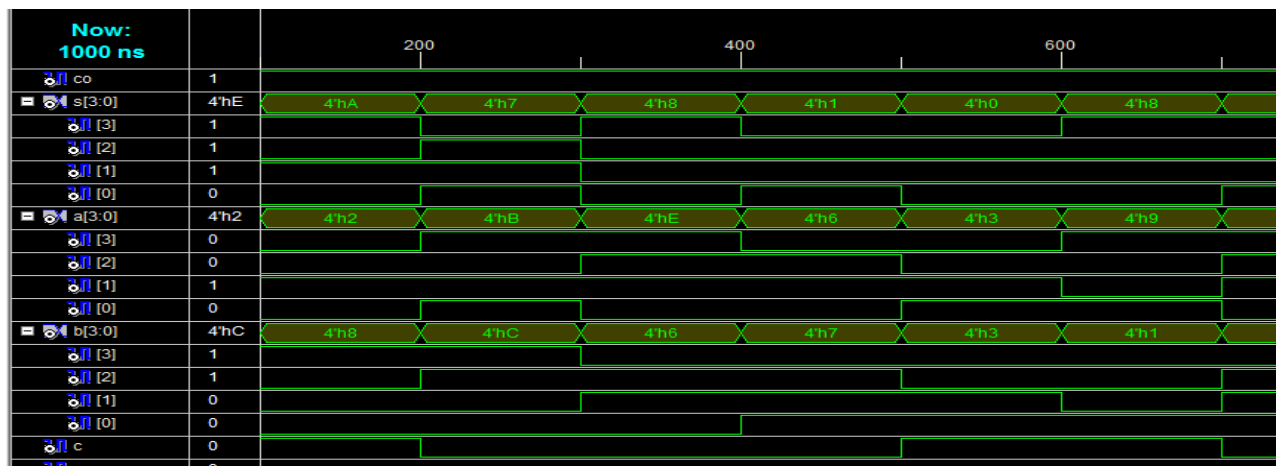

Figure 11. Proposed Design 2 Look ahead carry adder simulation results.

The above Figure shows the results of the proposed reversible look ahead carry adder for three different inputs. the inputs to the adder is two 4-bit binary coded decimal numbers named a and b and carry Cin. 


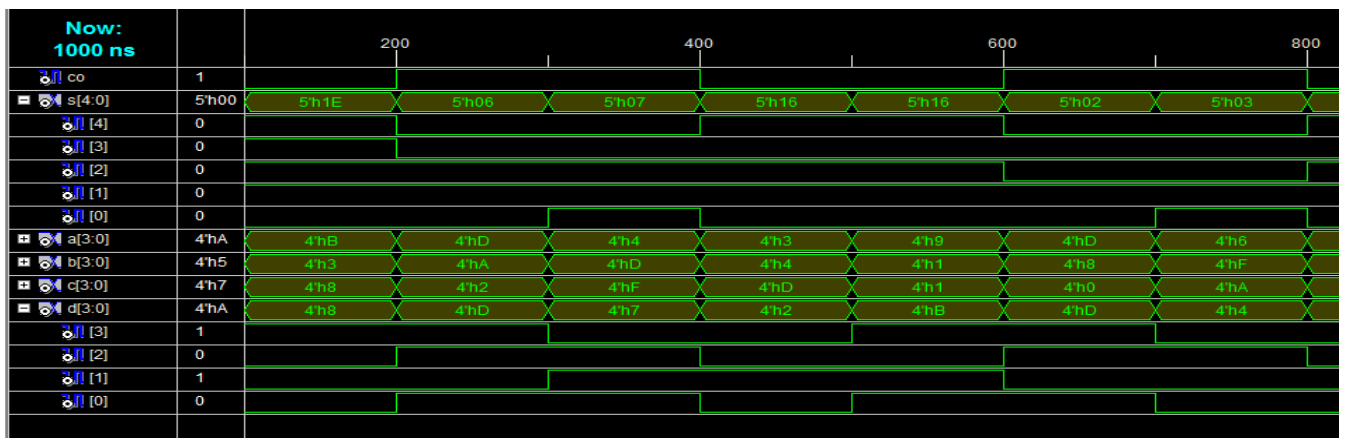

Figure 12. Proposed design 1 carry save adder simulation results.

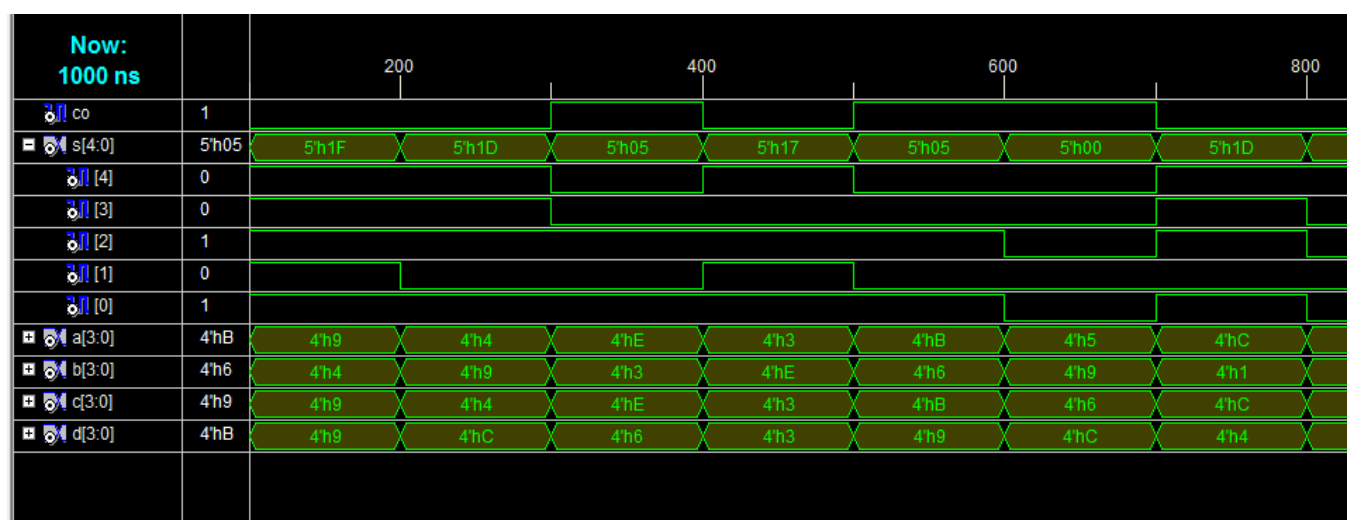

Figure 13. Proposed design 2 carry save adder simulation results.

\section{HARDWARE AND SOFTWARE USED}

All simulations have been done using Xilinx ISE 9.2i.

\section{APPLICATIONS}

Applications based on reversible logic concept are listed. (1) Nano Computing (2) Spacecraft

(3) Bio Molecular Computations (4) Quantum Computing (5) Low power CMOS. 


\section{CONCLUSION}

Look ahead carry adder is designed with reversible logic gates. Proposed design optimized with existing design in terms of reversible gate operations.

Table 3. Carry look ahead adder optimization results.

\begin{tabular}{|c|c|c|c|c|}
\hline Design & $\begin{array}{c}\text { No. of reversible } \\
\text { gates used }\end{array}$ & $\begin{array}{c}\text { No. of garbage } \\
\text { outputs }\end{array}$ & Quantum cost & Time delay \\
\hline Design 1 & 32 & 40 & 128 & $14.33 \mathrm{~ns}$ \\
\hline Design 2 & 20 & 20 & 72 & $12.275 \mathrm{~ns}$ \\
\hline
\end{tabular}

Table 4. Optimization table of carry save adder.

\begin{tabular}{|c|c|c|c|c|}
\hline Design & $\begin{array}{c}\mathbf{N}^{\circ} \text {.of reversible } \\
\text { gates used }\end{array}$ & $\begin{array}{c}\mathbf{N}^{\circ} \text {.of garbage } \\
\text { outputs }\end{array}$ & Quantum cost & Time delay \\
\hline Design 1 & 24 & 24 & 96 & 19.219 ns \\
\hline Design 2 & 12 & 24 & 72 & 15.873 ns \\
\hline
\end{tabular}

Reversible circuit design strategy is used to reduce the complexity and circuit cost. Distinguish the circuit with reversible systems, which performs more number of complex operations. Based on the reversible circuit design main factors like garbage outputs, quantum cost and delay of the circuit reduced. In addition, circuit complexity reduced by reducing the reversible gates. This work customs basic step in constructing complicated reversible systems, which can perform more comple x operations. Based on logical synthesis alternative design can be implemented. To decrease system design and manufacturing cost VLSI system implemented with one type of modular building. To reduce quantum cost and gates count, further design can be implemented using other reversible logic gates. 


\section{REFERENCES}

Agarwal, K. K., Choudhary, M. P., Jangid, H. K., \& Kasera, A. (2017). Design and implementation of reversible carry look ahead adder and array multiplier. International Journal of Advanced Research in Basic Engineering Sciences and Technology (IFARBEST), 3(Special Issue 23), 34-37.

Haque, M. U., Sworna, Z. T., \& Babu, H. M. H. (2016). An Improved Design of a Reversible Fault Tolerant LUT-based FPGA. In 29th International Conference on VLSI Design and 2016 15th International Conference on Embedded Systems (VLSID). Kolkata, India. IEEE. https://doi.org/10.1109/VLSID.2016.39

Lisa, N. J., \& Babu, H. M. H. (2015). Design of a Compact Reversible Carry LookAhead Adder Using Dynamic Programming. In 28th International Conference on VLSI Design. Bangalore, India. IEEE. https://doi.org/10.1109/VLSID.2015.46

Prashanth, N. G., Savitha, A. P., Anadaraju, M. B., \& Nuthan, A. G. (2103). Design and Synthesis of Fault Tolerant Full Adder/Subtractor Using Reversible Logic Gates. International Journal of Engineering Research and Applications (IJERA), 3(4), 137142. https://www.ijera.com/papers/Vol3_issue4/W34137142.pdf

Rajesh, K., \& Umamaheswara, G. (2017). Implementation of Ripple Carry and Carry Look Ahead Adders Using Reversible Logic Gates. International Fournal of Applied Engineering Research, 12(3), 3665-3670. https://www.researchgate.net/ publication/322481988_FPGA_implementation_of_ripple_carry_and_carry_look_ ahead_adders_using_reversible_logic_gates

Rajmohan, V., \& Ranganathan, V. (2011). Design of counter using reversible logic. In 3rd International Conference on Electronics Computer Technology. Kanyakumari, India. IEEE. https://doi.org/10.1109/ICEGTECH.2011.5941973

Rangaraju, H.G., Venugopal, U., Muralidhara, K.N., \& Raja, K.B. (2010). Low Power Reversible Parallel Binary Adder/Subtractor. International journal of VLSI design E Communication Systems, 1(3), 23-34. https://doi.org/10.5121/vlsic.2010.1303 
Safari, P., Haghparast, M., \& Azari, A. (2012). A Design of Fault Tolerant Reversible Arithmetic Logic Unit. Life Science Journal, 9(3). https://www.researchgate.net/ publication/230642890_A_Design_of_Fault_Tolerant_Reversible_Arithmetic_ Logic_Unit

Sayem, A.S.M., \& Ueda, M. (2010). Optimization of reversible sequential circuits. Fournal of computing, 2(6). https://pdfs.semanticscholar.org/4918/6fbed8b la3139fce9be426 cb4bbd0f073a3c.pdf

Somani, N., Ghaudhary, G., \& Yadav, S. (2016). Reversible Adder Design For Ripple carry and Carry look ahead. In International Conference on Computing, Communication and Automation (ICCCA). Noida, India. IEEE. https://doi.org/10.1109/ CCAA.2016.7813935

Thapliyal, H., \& Ranganathan, N. (2010). Design of Reversible Sequential Circuits Optimizing Quantum Cost, Delay, and Garbage outputs. ACM Journal on Emerging Technologies in Computing Systems, 6(4). https://doi.org/10.1145/1877745.1877748 


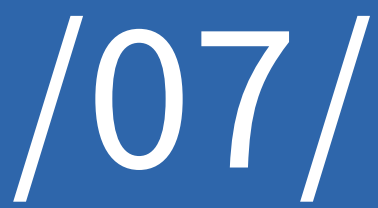




\section{ENHANCING UNDERWATER IMAGES USING PIECEWISE LINEAR SMOOTHING GRADIENT GUIDED FILTER}

A. Chrispin Jiji

Assistant Professor, Department of Electronics and Communication Engineering, The Oxford College of Engineering, Bangalore-560068 and affiliated to Visvesvaraya Technological University, Belagavi, Karnataka, (India). E-mail: chrispinjij@gmail.com ORCID: https://orcid.org/0000-0001-5267-788X

N. Ramrao

Vice Chancellor, Kalasalingam University, Srivilliputtur, Tamilnadu, (India). E-mail: nagaraj.ramrao@gmail.com ORCID: https:// orcid.org/0000-0003-2542-5999

\section{Citación sugerida:}

Chrispin Jiji, A., y Ramrao, N. (2020). Enhancing underwater images using piecewise linear smoothing gradient guided filter. 3C Tecnología. Glosas de innovación aplicadas a la pyme. Edición Especial, Marzo 2020, 129-139. http://doi.org/10.17993/3ctecno.2020.specialissue4.129-139

\section{Suggested citation:}

Chrispin Jiji, A., \& Ramrao, N. (2020). Enhancing underwater images using piecewise linear smoothing gradient guided filter. 3C Tecnología. Glosas de innovación aplicadas a la pyme. Edición Especial, Marzo 2020, 129-139. http://doi.org/10.17993/3ctecno.2020.specialissue4.129-139 


\section{ABSTRACT}

Poor visibility owing towards illumination absorption and scattering is challenging for processing undersea descriptions. However, for enhancing true scene from such degradation is more important. Unfortunately, existing methods cause gradient reversal artifact particularly near boundaries. To get better insight of undersea imagery, we project a piecewise linear smoothing Gradient Guided Filter (P-GGF) technique is to defeat the difficulties caused by conventional schemes, hence produce sharper boundaries based on GGF and smoothed output based on piecewise linear model. The projected technique mainly functional for smoothing, flash and feathering. Tentative results prove that the resultant algorithm can produce imagery with improved ocular excellence than existing methods.

\section{KEYWORDS}

Image Enhancement, Guided Filter, Piecewise liner smoothing, Piecewise constant smoothing. 


\section{INTRODUCTION}

Discovering an unexplained undersea globe has paying attention in modern era. Clear descriptions in oceanic surroundings take part a significant task in discovering as well as inspecting underneath globe, namely to observe oceanic biodiversity, undersea salvage, perceiving submerged tube drip, undersea computer visualization applications etc. Here the submerged imaging finds a large-scale application. Some difficulty comes all throughout underneath descriptions of illumination absorption as well as scattering. Deep-rooted design of ocean also composes complications in undersea. On the design of the ocean, reflection of the illumination alters. The reflected radiance is horizontally polarized along with its halfway gets inside the water vertically. Vertical polarization has significant property that hatches the substance not as great shining and aides on the way to capture deep colors. One more difficulty in underneath descriptions associated towards underneath density as 800 bits impenetrable than air. Hence when beam travels from air into water, it is halfway back and, in the meantime, partially enters the water. As we go deeper into ocean, dimension of beam underneath starts reducing. The underneath molecules absorb assertive size of beam and create problem for capturing imagery. That is the reason; undersea descriptions are getting darker as depth increases. The color with shorter wavelength travels remoteness as compared to longer wavelength. This is why undersea descriptions conquered only with blue color as in Torres-Méndez and Dudek (2005), Chiang and Chen (2012).

There are many challenges that enhance visibility of corrupted descriptions. While weakening of submerged descriptions outcomes the combination of multiplicative as well as additive procedures in Schettini and Corchs (2010) conventional improvement system namely contrasts alteration, histogram equalizer is robustly defective for such assignment. Former mechanism to review in section II, difficulty was attempted with customized attainment strategy by several imagery in Narasimhan and Nayar (2003), specific module in He and Seet (2004) or divergence methods in Schechner and Averbuch (2007). Regardless of their accomplishment, above approaches undergo various problems which degrade the system performance.

In contrast, this paper proposes new method for enhancing undersea descriptions using Piecewise linear smoothing. Our approach uses Piecewise linear smoothing gradient 
guided filter for getting better picture. Numerous spatial field schemes use bilateral scheme, which causes blurring and gradient deformation. Gradient Guided representation performs filtering process using guidance picture substance. Thus, a boundary preservation method mainly to improve excellence of underneath descriptions.

This paper prepared as follows. Foremost, in Section 2, we concise the existing schemes. Section 3 introduces a comparison of conventional and projected filters. Section 4 describes a new method for enhancing undersea descriptions. In Section 5, we describe tentative outcomes and at last Section 6 conclude our method.

\section{RELATED WORK}

Edge-preserving smoothing is the fundamental processing procedure within several lowlevel computer visualization applications in Farban, Fattal and Lischinski (2010), Farbman, Fattal, Lischinski, and Szeliski (2008), Gastal and Oliveira (2011; 2012). Meant for on whole smooth filters believe the smoothed output imagery are piecewise constant. Generally, the edge-preserving techniques using confined filtering to keep sharp boundaries. Bilateral filter is extensively used because of its effortlessness Tomasi and Manduchi (1998). Conversely, it undergoes unwanted sharpening of edges may show undesired profiles around edges. Guided filter introduced in He, Sun, and Tang (2013) overcome these problems but show unwanted smoothing edges. Weighted GF in Li et al. (2015) uses gradient-domain constrains for smoothing the picture elements but in few cases, it cannot preserve the boundaries. The gradient domain GIF in Kou, Chen, Wen, and $\operatorname{Li}$ (2015) incorporates a precise initial-order boundary-aware restraint to keep up boundaries better in some cases.

These conventional schemes are typically denoted as local model which causes artifact such as gradient reversals, hence may not fine for few cases. For those schemes, a piecewise linear form preferred mostly for properly smooth out boundaries. So, no artifacts are present in improved results. In Liu et al. (2018) piecewise linear method via guided representation accurately resolve difficulty of gradient-reversal except that only some cases illustrate small smoothing boundaries.

Therefore, we project a P-GGF to properly sharp, smooth all boundaries as well as do artifacts free enhanced result. Three major goals of projected sections as follows: 
1. To take away gradient reversal, we use Gradient Guided Filter (GGF) to give sharper boundaries.

2. Next, we use piecewise linear smoothing to smooth the boundaries.

3. The projected module uses P-GGF to produce improved output.

Experimental results produced by P-GGF can perfectly remove the problems caused by the conventional method.

\section{PIECEWISE LINEAR SMOOTHING}

Conventional methods mostly suitable for image regions more likely be piecewise linear may cause artifacts. Clearly, detail layers cannot correctly say details in the original descriptions. Thus, gradient reversal artifacts exist in their enhanced imagery. In highlighted regions, two kinds of smoothing can properly keep strong gradients which means strong edges are properly preserved. For weak edges of small gradients which should be smoothed out. However, these gradients are not properly smoothed by conventional methods or even improperly sharpened. As a result, gradient reversal artifacts exist with the enhanced representation.

For most cases, conventional methods usually need a huge number of bins to avoid quantization artifacts. Smoothed and enhanced descriptions achieved with first smoothing of conventional filters and later uses smooth gradient for reconstructing the image. The reason for these phenomena is clear. The classical smoothing performed in intensity domain where intensity values could be very large. In contrast, to overcome all problems we go for proposed method namely piecewise linear method.

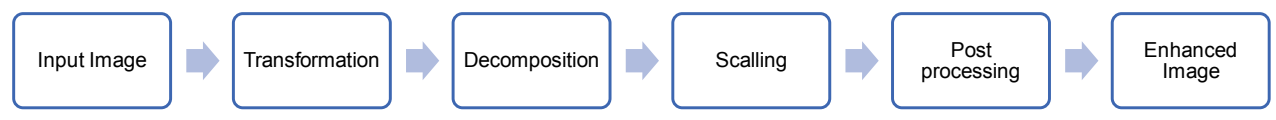

Figure 1. Block diagram of Proposed Method. 


\section{PROPOSED METHOD}

As depicted in Figure 1 our technique is mainly for enhancing underwater images using piecewise linear smoothing. Initially, original picture developed by RGB or HSV color space. RGB depict colors in combination of primary colors.

HSV describes colors namely Hue, Saturation, and Value. Color depiction acting an essential task, HSV form often chosen over RGB form and express color likewise to how human vision lean-to recognize color. Later, we used first layer decomposition by smoothing the image using L1L0 smoothing and second layer decomposition for sharpening the edges using gradient guided filter. Next, scaling to vary ocular form of a picture. Finally, post processing of scaled image to enhance the quality of the image.

The conventional method can simply modeled as:

$$
E_{P}^{\wedge}=a_{k}, P \in N(k)
$$

where $E_{P}^{\wedge}$ represents expected output pixel rate at location $P=\left[P_{x}, P_{y}\right], a_{k}$ denotes expected constant rate of pixel values inside kth area in the picture indicated as $N(k)$. In contrast to conventional model assumption, projected method can signify:

$$
E_{P}^{\wedge}=a_{k} G_{P}+b_{k}, P \in w(k)
$$

where $E_{P}^{\wedge}$ denotes expected output pixel value at position $\mathrm{p}, G_{P}$ represents pixel value of guidance image at position p. $a_{k}$ and $b_{k}$ stay steady in $w(k)$. These methods show no gradient reversal artifacts.

In this paper, we assume a linear form which is spatially linear different from gradient guided image filter. In addition, our method also focuses on how to do projected method using classical methods.

Different from piecewise linear function, we formulate imagery as:

$$
E_{P}^{\wedge}=a_{k}^{T} P+b_{k}, P \in N(k)
$$


where $a_{k}=\left[a_{k}^{x}, a_{k}^{y}\right]^{T}$ as well as $b_{k}$ denotes some linear coefficients which assumed to stay constant inside $N(k)$. The above equation indicates linear polynomial regression to remove stair-casing effect. The model in (3) is general and abstract form which is not used for explicit formulations of filters. However, when we take the derivative of $E_{P}^{\wedge}$ with respect to $\mathrm{p}$, then we have:

$$
\frac{\partial E_{P}^{\wedge}}{\partial P}=a_{k}, P \in N(k)
$$

Note that $\frac{\partial E_{P}^{\wedge}}{\partial P}$ signifies gradient of picture at p. For linear form, their gradients are piecewise constant. Though, dissimilarity assumes resultant imagery of smoothed output gradients. Conversely, problem is that we cannot simply reconstruct picture only by its smoothed gradients. The difficulty in using original and its filtered gradients is to reconstruct filtered image. For original picture, its x-axes and y-axes gradients denoted as $\partial x I_{0}$ and $\partial y I_{0}$. By denoting smoothing process of piecewise constant filters as $\mathfrak{\Im}_{p c}($.$) , then final representation$ $E$ reconstructed by minimizing the following energy function:

$$
\left\|\hat{E}-E_{0}\right\|^{2}+\beta\left\{\left\|\partial x \hat{E}-\mathfrak{J}_{p c}\left(\partial x E_{0}\right)\right\|^{2}-\left\|\partial y \hat{E}-\mathfrak{J}_{p c}\left(\partial y E_{0}\right)\right\|^{2}\right\}
$$

From this, we can execute piecewise linear smoothing through conventional scheme in the following two steps:

(1). Smoothing x-axes in addition to y-axes, gradients $\partial x E_{0}$ and $\partial y E_{0}$ of original representation $E_{0}$ with $\mathfrak{I}_{p c}($.$) . The smoothed output gradients are denoted by \mathfrak{I}_{p c}\left(\partial x E_{0}\right)$ and $\mathfrak{I}_{p c}\left(\partial y E_{0}\right)$

(2). Using (5) for enhancing picture $\hat{E}$ from $E_{0}, \mathfrak{I}_{p c}\left(\partial x E_{0}\right)$ and $\mathfrak{I}_{p c}\left(\partial y E_{0}\right)$ with a proper value of $\beta$. Then the enhanced $\hat{E}$ is spatially piecewise linear as modelled in (3).

\section{EXPERIMENTAL RESULTS}

This section presents a comparison of improved outcome produced via ours and conventional schemes through subjective evaluation of test scene. Figure 2 gives improved result than conventional methods. As seen from Figure 2(a)-(f), all schemes get better output to some 
extent. Figure 2(a) bilateral process Tomasi and Manduchi (1998) results enhanced scene, but it cannot keep sharp boundaries. In Figure 2(b) He et al. (2013) discussed Guided filter to keep boundaries sharper but fail to represent the picture well close to a few boundaries. To defeat Li, Zheng, Zhu, Yao and Wu (2015) used weighted guided scheme in Figure 2(c) used for minimizing halo artifacts but fail to keep the boundaries.

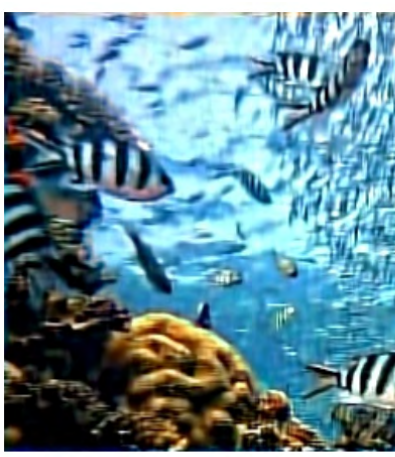

a) (Tomasi and Manduchi, 1998)

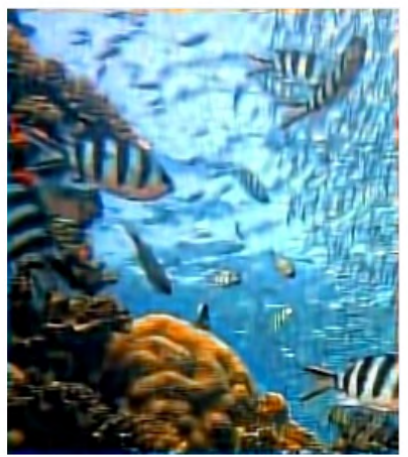

d) (Kou et al., 2015)

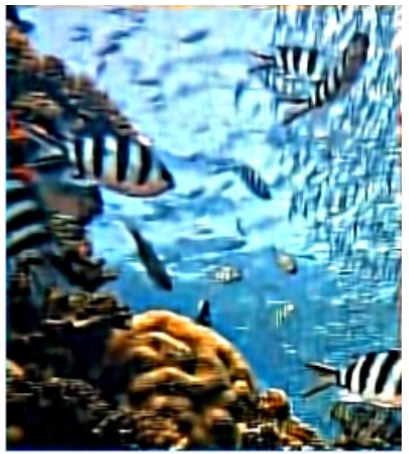

b) (He et al., 2013)

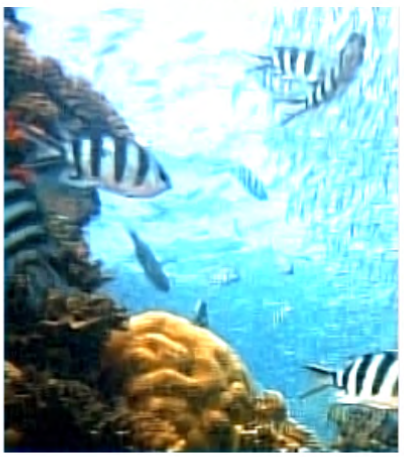

e) Liu et al. (2018)

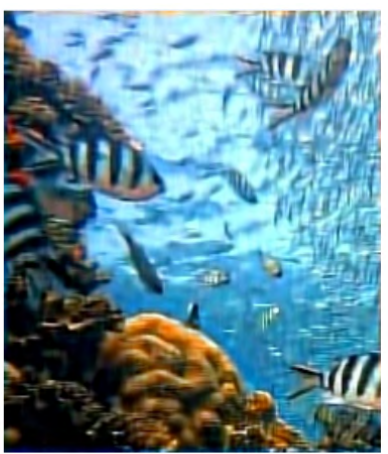

c) (Li et al., 2015)

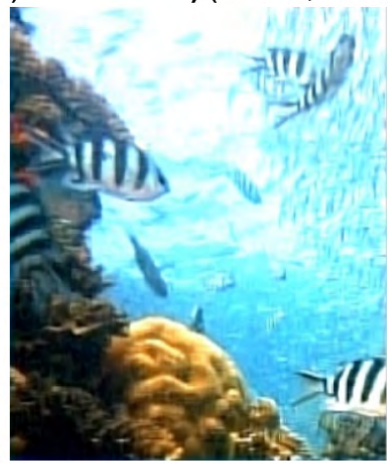

f) Own elaboration

Figure 2. Enhancing performance evaluation of Underwater Imagery.

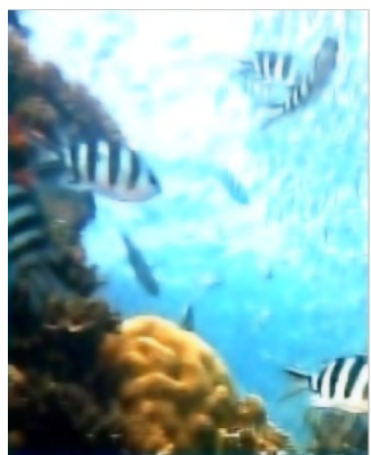

a)

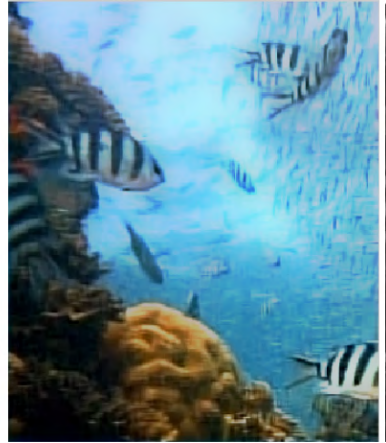

b)

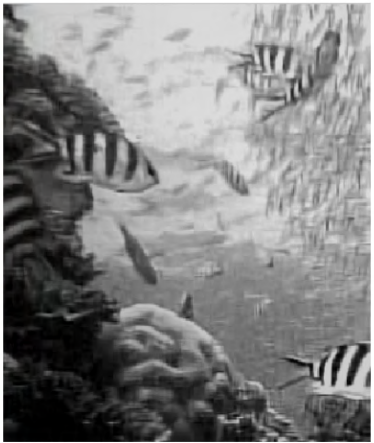

c)

Figure 3. Various applications of Proposed Method a) Smoothing b) Flash c) Feathering. 
Figure 2(d), Kou et al. (2015) used an edge aware factor introduced to keep up well-built boundaries but smoothed away weak boundaries. Piecewise linear method via guided representation Liu et al. (2018) in Figure 2(e) accurately resolve difficulty of gradient-reversal except that only some cases illustrate small smoothing boundaries. At last projected approach Figure 2(f) outcomes strong and weak boundaries accurately and makes the standardized system into range $[0 ; 1]$ earlier to smoothing and then standardize back towards original range after smoothing. Figure 3 gives various applications of projected scheme to smooth out weak boundaries, drop artifacts using flash filtering and refine boundaries using feathering.

However, the projected scheme gives improved results than the schemes in Tomasi and Manduchi (1998), He et al. (2013), (Li et al., 2015), Kou et al. (2015), Liu et al. (2018). Therefore, projected scheme is more suitable for various applications.

\section{CONCLUSIONS}

We projected a piecewise linear smoothing Gradient Guided Filter (P-GGF) method to improve undersea descriptions. The proposed method properly handles gradient reversal artifacts caused by conventional and uses GGF with sharper boundaries and smoothed result based on piecewise linear model. Overall, projected scheme P-GGF can effectively improve the scene. Experimental results prove that projected method generate imagery by improved ocular excellence than conventional methods. We believe that proposed technique used for many applications such as smoothing, Flash and feathering. The extra remarkable difficulty based on the expansion of the projected method to obtain fine details from numerous descriptions concurrently using extensive filter in Li et al (2012; 2014). We leave this for future research.

\section{ACKNOWLEDMENT}

We gratefully thank the Visvesvaraya Technological University, Jnana Sangama, Belagavi for financial support extended to this Research work. 


\section{REFERENCES}

Ghiang, J. Y., \& Chen, Y. G. (2012). Underwater image enhancement by wavelength compensation and dehazing. IEEE Transactions on Image Processing, 31(4), 1756-1769. https://doi.org/10.1109/TIP.2011.2179666

Farban, Z., Fattal, R., \& Lischinski, D. (2010). Diffusion maps for edge-aware image editing. ACM Transa Grap. https://www.cse.huji.ac.il/labs/cglab/projects/diffmaps/ diffmaps.pdf

Farbman, Z., Fattal, R., Lischinski, D., \& Szeliski, R. (2008). Edge-preserving decompositions for multi-scale tone and detail manipulation. ACM Transactions on Graphics, 27(3). https://doi.org/10.1145/1360612.1360666

Gastal, E. S. L., \& Oliveira, M. M. (2011). Domain transforms for edge awareimage and video processing. ACM Transactions on Graphics, 30(4). https://doi. org/10.1145/2010324.1964964

Gastal, E. S. L., \& Oliveira, M. M. (2012). Adaptive manifolds for real-time high-dimensional filtering. ACM Transactions on Graphics, 31(4). https://doi. org/10.1145/2185520.2185529

He, D. M., \& Seet, G. G. L. (2004). Divergent-beam LiDAR imaging in turbid water. Optics and Lasers in Engineering, 41(1), 217-231. https://doi.org/10.1016/S01438166(02)00138-0

He, K., Sun, J., \& Tang, X. (2013). Guided image filtering. IEEE Transactions on Pattern Analysis and Machine Intelligence, 35(6), 1397-1409. https://doi.org/10.1109/ TPAMI.2012.213

Kou, F., Ghen, W., Wen, G., \& Li, Z. (2015). Gradient Domain Guided Image Filtering. IEEE Transactions on Image Processing, 24(11). https://doi.org/10.1109/ TIP.2015.2468183 
Li, Z., Zheng, J., Zhu, Z., Yao, W., \& Wu, S. (2015). Weighted guided image filtering. IEEE Transactions on Image Processing, 24(1), 120-129. https://doi.org/10.1109/ TIP.2014.2371234

Li, Z. G., Zhe, J. H., \& Rahardja, S. (2012). Detail-enhanced exposure fusion. IEEE Transactions on Image Processing, 21(11). https://doi.org/10.1109/TIP.2012.2207396

Li, Z.G., Zheng, J., Zhu, Z., \& Wu, S. (2014). Selectively detail enhanced fusion of differently exposed images with moving objects. IEEE Transactions on Image Processing, 23(10). https://doi.org/10.1109/TIP.2014.2349432

Liu, W., Xu, W., Ghen, X., Huang, X., Shen, G., \& Yang, J. (2018). Edge Preserving Piecewise Linear Smoothing using Piecewise Constant Filters. arXiv:1801.06928. https://arxiv.org/abs/1801.06928

Narasimhan, S. G., \& Nayar, S. K. (2003). Contrast restoration of weather degraded images. IEEE Transactions on Pattern Analysis and Machine Intelligence, 25(6),713- 724. https://doi.org/10.1109/TPAMI.2003.1201821

Schechner, Y. Y., \& Averbuch, Y. (2007). Regularized image recovery in scattering media. IEEE Transactions on Pattern Analysis and Machine Intelligence, 29(9), 1655-1660. https:// doi.org/10.1109/TPAMI.2007.1141

Schettini, R., \& Corchs, S. (2010). Underwater Image Processing: State of the Art of Restoration and Image Enhancement Methods. EURASIP Journal on Advances in Signal Processing, 746052. https://doi.org/10.1155/2010/746052

Tomasi, G., \& Manduchi, R. (1998). Bilateral filtering for gray and color images. In Proceedings of the 1998 IEEE International Conference on Computer Vision, Bombay, India. https://users.soe.ucsc.edu/ manduchi/Papers/ICGV98.pdf

Torres-Méndez, L. A., \& Dudek, G. (2005). Color Correction of Underwater Images for Aquatic Robot Inspection. In Rangarajan A., Vemuri B., \& Yuille A.L. (eds). Energy Minimization Methods in Computer Vision and Pattern Recognition. EMMCVPR 2005. Lecture Notes in Computer Science, vol. 3757. Springer, Berlin, Heidelberg. https://doi. org/10.1007/11585978_5 


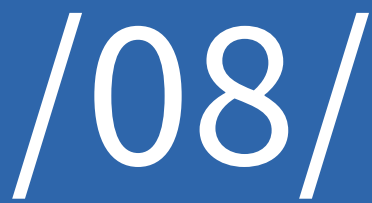




\section{THE HUMAN EAR RECOGNITION BASED ON PHASE- BASED MATCHING ALGORITHM}

Muthukumar Arunachalam

Department of Electronics and Communication Engineering Kalasalingam Academy of Research and Education. Krishnankoil, (India). E-mail: muthuece.eng@gmail.com ORCID: https://orcid.org/0000-0001-8070-3475

\section{Citación sugerida:}

Arunachalam, M. (2020). The human ear recognition based on phase-based matching algorithm. 3C Tecnología. Glosas de innovación aplicadas a la pyme. Edición Especial, Marzo 2020, 141-157. http://doi. org/10.17993/3ctecno.2020.specialissue4.141-157

\section{Suggested citation:}

Arunachalam, M. (2020). The human ear recognition based on phase-based matching algorithm. 3C Tecnología. Glosas de innovación aplicadas a la pyme. Edición Especial, Marzo 2020, 141-157. http://doi. org/10.17993/3ctecno.2020.specialissue4.141-157 


\section{ABSTRACT}

In this modern era, biometric play a vital role in authentication process for an individual identification. Broadly biometrics has been classified into anatomical and behavioral characteristics. Among the many biometrics, this paper focus on new emerging biometric named as Ear biometrics. In this paper, Phase-only Correlation (POC) and Band-limited Phase Only Correlation (BLPOG) has been proposed for ear recognition. The phase information has been obtained by calculating similarity between input and query ear image using 2D-Discrete Fourier Transform (DFT) and auto correlation function. Finally the phase-based on image matching have being success implemented for human ear recognition endeavor. The experimental resultant effect of proposed algorithm has been performed using IIT Delhi ear database.

\section{KEYWORDS}

DFT, Phase Only Correlation (POG), Band-Limited Phase Only Correlation (BLPOC). 


\section{INTRODUCTION}

In impressive of last few years, Human Ear recognition has been becoming a very attractive in biometric authentication. The important reasons behind human ear biometric over other biometric modalities are smaller in size, very stable shape has proven by clinical observation (Rutty, Abbas, \& Crossling, 2005), uniform colors, and does not affected by any expression like face or changes in age or position or rotation (Malathy, Annapurani, \& Sadiq, 2013). The ear is a passive biometric as it can easily be captured from far distance, even if the person is not fully cooperative. Hence ear recognition is much interest for the researchers dealing with numerous applications in commercial and governmental, such as law enforcement, security systems and forensics (Jain, Ross, \& Pankanti, 2006). Therefore, ear biometric is a deserved preference for it providing a fine avail between cost and accuracy.

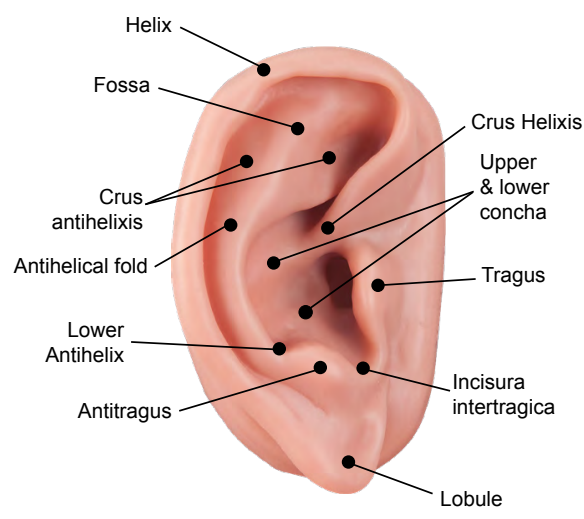

Figure 1. Ear Image.

The Figure 1 shows the ear image of an individual. It is stored in the IIT Delhi database and can be used for the task of identification through its unique characteristics. The human outer ears parts are formed by various terminologies include Helix, the Lobe, Antihelix, the tragus, concha along with an antitragus.

Recently, several matching algorithms have been adapted for the ear recognition process. Anyhow, these algorithms possess various limitations which are explained in this section. Primarily, the holistic matching method utilized from global features to extract the complete ear. Bustard et al. arrange the ear dataset by considering the planar surface. It was registered as homography transform designed of Scale Invariant Feature Transform from trails 
(Bustard \& Nixon, 2010). This method was strong to conditions in the appearance of pose variation and occlusion.

Ultimately, the matching algorithms with local feature have been adopted for identification. So Nanni et al. determined to decrease the dimension by usual sub-window of feature extraction with a Gabor Filter along with LaplacianEigenmaps (Zhu, Jia, \& Liu, 2009). They also used the Sequential Forward Floating Selection to sort out the finest matching feature with concord trained. However the function of Laplacian Eigen maps could drop datas extremely. Yazdanpanah et al. designed a region of covariance matrix through wellorganized plus strong ear caption, which is strong to clarification with fake difference (Miyazawa, Ito, Aoki, Kobayashi, \& Katsumata, 2006). In the following year, Yuan et al. ear region has extracted to segment by conserving from neighborhood impact and the particular region has chosen by the recognition rate (Zhang, Zhang, \& Zhang, 2009). This method could keep away from the occlusion problem other than shortcoming from multifake problem.
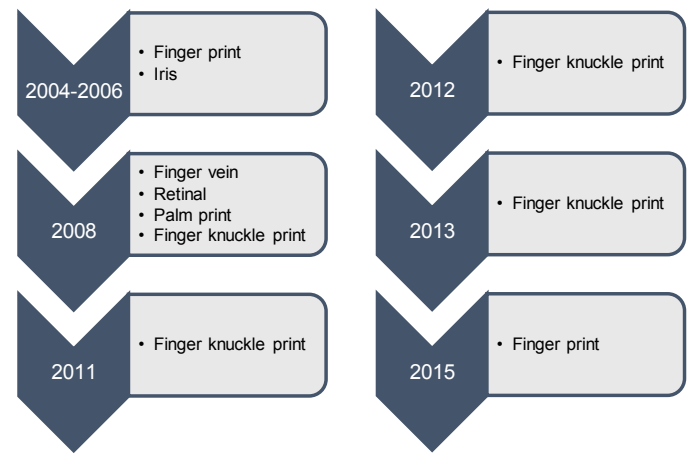

Figure 2. Different biometrics images used by POC and BLPOC.

The POC and BLPOC matching algorithm play the major role in image processing or pattern matching. There are many benefits in using Phase based matching algorithm such as simple to construct score, easy to implement, finest for multiple test and close to realtime performance. The Figure 2 shows different biometrics images are used by POC and BLPOC algorithm over the years for instance finger vein, finger print, iris, retinal, palm print as well as finger knuckle print except ear biometric. Therefore, this paper proposes human ear recognition based on phase-base matching algorithm. 


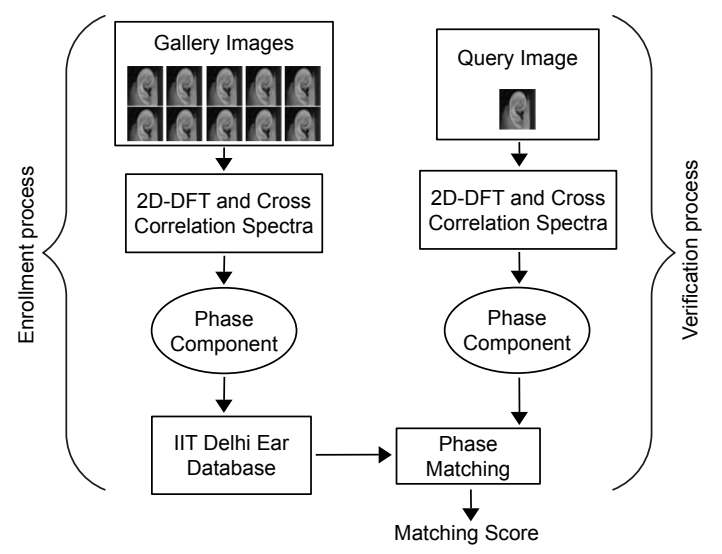

Figure 3. Proposed system.

The Figure 3 shows the proposed system of this paper. This proposed system involves two processes namely (i) Enrollment process and (ii) Verification process. First, enrollment process carries out the process that involves feature extraction of POC and BLPOC technique. This feature extraction minimizes the ear images into mathematical phase information. This information will be stored in database to establish the authentication of ear images. At the same time, verification process also arrive the same process for extracting features of POC and BLPOC technique which generates the phase information. This feature extraction converts the ear images into mathematical phase information. These both phase information's are afforded by decision making subsidiary. The decision making subsidiary used to provide a final conclusion (i.e. genuine or imposter output). The output of decision making is a potential matching score by matching identities.

The remainders in this paper are structured as follows: The second section discusses about survey of the literature which gives brief discussion about existed POG and BLPOC. The third section outlines the proposed work and its implementation of Human Ear Recognition. The fourth section discusses about presents the experimental results of proposed system. The conclusion has been described in the section 5 .

\section{RELATED WORK}

In this related work, discusses the detailed functions of phase-base on image matching algorithms (i.e. POG and BLPOC). The POC has been designed to find the correlation 
within two images (i.e. different images or similar images). The purpose of POC is to frame-up only phase feature and to isolate the magnitude feature of images for accuracy in image matching. During the trial time of POC with two similar images provides distinct sharp correlation peak and the correlation peak drops when two images are different (Takita, Muquit, Aoki, \& Higuchi, 2004). The experimental results of phase correlation demonstrate high robustness and accuracy in the pattern matching and the image registration (Ito, Nakajima, Kobayashi, Aoki, \& Higuchi, 2004). This has been achieved with direct estimation in Fourier domain through 2D phase difference data set (Miyazawa et al., 2006). This kind of POG correlation is more convenient and more profitable than other conventional correlation. The most remarkable advantages of POC are it provides better discrimination for similar images and high accuracy translational of displacement among the illustrations (Nanni \& Lumini, 2007). But for most part of phase function algorithm cannot carry the nonlinear deformation of illustrations (Rutty et al., 2005). The BLPOC is very crucial method for correlation in optimum band limit for phase based recognition. In POC function, every frequency components are concerned. Though, high frequency components tend to highlight and it probably prone to noise. Hence, worthless high frequency components are eliminated by setting a band-limited while calculating the cross-power spectrum (Yazdanpanah \& Faez, 2010). Also, in order to carry the nonlinear deformation, it employs BLPOC matching algorithm. The BLPOG be sufficient to estimate accurate overall correlation (Yuan, Wang, \& Mu, 2010). The modified version of POG is BLPOG which is committed to evaluate match between images due to its better performance than the POC function (Zhang, Zhang, Zhang, \& Guo, 2012). The proposed BLPOC perform (Zhang, Zhang, Zhang, \& Zhu, 2011) committed to biometric recognition tasks. Several authors attempted different modalities of biometric recognition by using POC and BLPOC are outlined in the given Table 1.

Table 1. Outline about Phase-Based Function matching algorithm in various biometrics.

\begin{tabular}{|c|c|c|}
\hline Author & Feature & Trait (Images) \\
\hline $\begin{array}{c}\text { (Takita et al., 2004). } \\
\text { (Ito et al., 2004). }\end{array}$ & POC and BLPOC & Finger Print \\
\hline $\begin{array}{c}\text { (Miyazawa, Ito, Aoki, } \\
\text { Kobayashi, \& Nakajima, } \\
\text { 2005). }\end{array}$ & POC and BLPOC & Iris \\
\hline (Zhu et al., 2009). & POC and BLPOC & Palm print \\
\hline
\end{tabular}




\begin{tabular}{|c|c|c|}
\hline Author & Feature & Trait (Images) \\
\hline $\begin{array}{c}\text { (Mahri, Suandi, \& Rosdi, } \\
\text { 2010). }\end{array}$ & POC and BLPOC & Finger Vein \\
\hline $\begin{array}{c}\text { (Miyazawa et al., 2006). } \\
\text { (Yuan et al., 2010). }\end{array}$ & POC and BLPOC & Retinal \\
\hline $\begin{array}{c}\text { (Zhang et al., 2011). } \\
\text { (Zhang et al., 2012). }\end{array}$ & POC and BLPOC & FKP \\
\hline $\begin{array}{c}\text { (Aoyama, Ito, \& Aoki, } \\
\text { 2014). }\end{array}$ & POC and BLPOC & FKP \\
\hline
\end{tabular}

Human Ear Recognition Using Phase-Based Function Matching.

\section{METHOD}

In this section, POC and BLPOC are discussed and derived in detail.

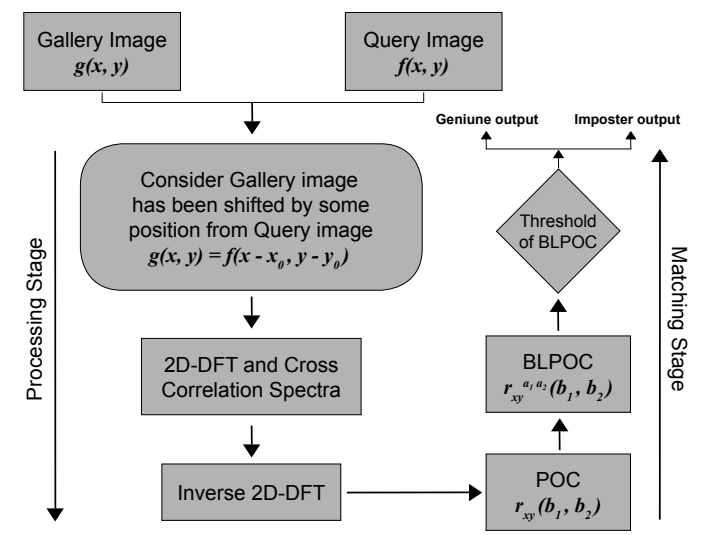

Figure 4. Overall structural outline of the proposed design of this paper.

The Figure 4 shows the overall structural outline of proposed design of this paper. This method is discussed in next section 3.1 and 3.2 in detail.

\subsection{PHASE-ONLY FUNCTION/CORRELATION (POC)}

This section discussed about the principal of POC. The POC is some time called as "PhaseOnly Function") (Zhang et al., 2009). Assume $2 \mathrm{M}$ x N images, g $x_{1}, x_{2}$ and the $f\left(x_{1}, x_{2}\right)$, where consider that the basis ranges are $x_{1}=M \ldots . . M(M \geq 0)$ and $x_{2}=N \ldots . . N(N \geq 0)$ in support of mathematical ease, and therefore $M=2 M+1$ and $N=2 N+1$. The conversation can be simply general towards non-nullifying basis ranges by rule of 2 image sizes. Let $F\left(a_{l}\right.$, 
$\left.a_{2}\right)\left(\right.$ and $G\left(a_{1}, a_{2}\right)$ denote the 2D-DFTs of $g\left(x_{1}, x_{2}\right)$ and, correspondingly. According to the description of DFT (Zhang et al., 2009), $F\left(a_{1}, a_{2}\right)$ and $G\left(a_{1}, a_{2}\right)$ be known through

$$
\begin{gathered}
F\left(a_{1}, a_{2}\right)=\sum_{x=0}^{M-1} \sum_{y=0}^{N-1} f\left(x_{1}, x_{2}\right) \cdot e^{\left(-j 2 \pi\left(\frac{a_{1} x_{1}}{M}+\frac{a_{2} x_{2}}{N}\right)\right)} \\
G\left(a_{1}, a_{2}\right)=\sum_{x=0}^{M-1} \sum_{y=0}^{N-1} g\left(x_{1}, x_{2}\right) \cdot e^{\left(-j 2 \pi\left(\frac{a_{1} x_{1}}{M}+\frac{a_{2} x_{2}}{N}\right)\right)}
\end{gathered}
$$

Consider $g\left(x_{1}, x_{2}\right)=f\left(x_{1}-x_{0}, x_{2}-y_{0}\right)$

Consider image is shifted by $x_{0}$ and $y_{0}$ portion, then $x_{0}=y_{0}=0$ the both is same. Now applying spatial shifting property,

$$
\begin{gathered}
F T\left[f\left(x_{1}-x_{0}, x_{2}-y_{0}\right)\right]=\exp ^{\left(-j 2 \pi\left(\frac{a_{1} x_{0}}{M}+\frac{a_{2} y_{0}}{N}\right)\right)} F\left(a_{1}, a_{2}\right) \\
\therefore G\left(a_{1}, a_{2}\right)=e^{\left(-j 2 \pi\left(\frac{a_{1} x_{0}}{M}+\frac{a_{2} y_{0}}{N}\right)\right)} F\left(a_{1}, a_{2}\right)
\end{gathered}
$$

For finding Correlation of, using equation

$$
f\left(x_{1}, x_{2}\right) * h\left(x_{1}, x_{2}\right)=\sum_{m=0}^{M-1} \sum_{n=0}^{N-1} f(m, n) \cdot h\left(x_{1}+m, x_{2}+n\right)
$$

Correlation phase spectra

$$
R_{G F}\left(a_{1}, a_{2}\right)=\frac{G\left(a_{1}, a_{2}\right) \cdot F^{*}\left(a_{1}, a_{2}\right)}{\left|G\left(a_{1}, a_{2}\right) \cdot F^{*}\left(a_{1}, a_{2}\right)\right|}
$$

Sub 6 and 7, so we get,

$$
\therefore R_{G F}\left(a_{1}, a_{2}\right)=\exp ^{\left(-j 2 \pi\left(\frac{a_{1} x_{0}}{M}+\frac{a_{2} y_{0}}{N}\right)\right)}
$$

2DIDFT-

$$
f\left(x_{1}, x_{2}\right)=\frac{1}{M N} \sum_{u=0}^{M-1} \sum_{v=0}^{N-1} F\left(a_{1}, a_{2}\right) \exp \left(-j 2 \pi\left(\frac{a_{1} x_{1}}{M}+\frac{a_{2} x_{2}}{N}\right)\right)
$$

Take 2D-IDFT for equation 7 , 


$$
\therefore r_{g f}\left(x_{1}, x_{2}\right)=\frac{1}{M N} \sum_{u=0}^{M-1} \sum_{v=0}^{N-1} R_{G F}\left(a_{1}, a_{2}\right) \exp \left(-j 2 \pi\left(\frac{a_{1} x_{1}}{M}+\frac{a_{2} x_{2}}{N}\right)\right)
$$

Put equation 8 in 10,

$$
\begin{gathered}
r_{g f}\left(x_{1}, x_{2}\right)=\frac{1}{M N} \sum_{u=0}^{M-1} \sum_{v=0}^{N-1} \exp ^{\left(-j 2 \pi\left(\frac{a_{1} x_{1}}{M}+\frac{a_{2} x_{2}}{N}\right)\right)} \exp \left(-j 2 \pi\left(\frac{a_{1} x_{0}}{M}+\frac{a_{2} y_{0}}{N}\right)\right) \\
=\frac{1}{M N} \sum_{u=0}^{M-1} \sum_{v=0}^{N-1} \exp ^{-j 2 \pi\left[\frac{a_{1} x_{0}}{M}+\frac{a_{2} y_{0}}{N}-\frac{a_{1} x_{1}}{M}-\frac{a_{2} x_{2}}{N}\right]} \\
r_{g f}\left(x_{1}, x_{2}\right)=\frac{1}{M N} \sum_{u=0}^{M-1} \sum_{v=0}^{N-1} \exp ^{\left.-j 2 \pi\left[\frac{a_{1}\left(x_{0}-x_{1}\right)}{M}+\frac{a_{2}\left(y_{0}-x_{2}\right)}{N}\right)\right)} \\
x_{0}-x_{1}=0 \quad y_{0}-x_{2}=0 \\
r_{g f}\left(x_{1}, x_{2}\right)=1 \quad(\text { Peak value })
\end{gathered}
$$

Respectively, where $a_{1}=-M_{1} \ldots M_{1}, \quad a_{2}=-M_{2} \ldots M_{2}, W_{N 1}=e^{-j 2 \pi / N 1} \quad, W_{N 2}=e^{-j 2 \pi / N 2}$ and $\Sigma_{n 1 n 2}$ denotes $\Sigma_{n_{1}=-m_{1}}^{M 1} \Sigma_{n_{2}=-m_{2}}^{M 2} . \quad A_{F}\left(a_{1}, a_{2}\right)$ and $A_{G}\left(a_{1}, a_{2}\right)$ are amplitude, and $\theta_{F}\left(a_{1}, a_{2}\right)$ and $\theta_{G}\left(a_{1}, a_{2}\right)$ are phase. The normalized cross power spectrum $R_{F G}\left(a_{1}, a_{2}\right)$ is set by

$$
R_{F G}\left(a_{1}, a_{2)}=\mathrm{F}\left(a_{1}, a_{2)} \overline{G\left(a_{1}, a_{2}\right)} / \mid \mathrm{F}\left(a_{1}, a_{2}\right) \overline{G\left(a_{1}, a_{2}\right)}\right.\right.
$$

Where $G\left(a_{1}, a_{2}\right)$ is the complex conjugate of $G\left(a_{1}, a_{2}\right)$ and $\theta\left(a_{1}, a_{2}\right)$ denotes the phase difference $\theta_{F}\left(a_{1}, a_{2}\right)-\theta_{G}\left(a_{1}, a_{2}\right)$. The POC function $r_{f g}\left(n_{1}, n_{2}\right)$ is the 2D IDFT of $R_{F G}\left(a_{1}, a_{2}\right)$ and is set by

$$
r_{f g}\left(x_{1}, x_{2}\right) \frac{1}{N_{1} N_{2}} \sum_{k 1 k 2} R_{F G}\left(a_{1}, a_{2}\right) W_{N 1}^{-a_{1} n_{1}} W_{N 2}^{-a_{1} n_{2}}
$$

Where $\Sigma_{a_{1}, a_{2}}$ denotes $\Sigma_{a_{1}=-m 1}^{M 1} \Sigma_{a_{2}=-m 2}^{M 2}$. When 2 images are alike, their POC perform provides a definite quick peak. When 2 images aren't alike, the height drop noticeably. The height of peak provides a decent likeness for match the image, with therefore the position of height shows change of location displacement among the illustration (Zhang et al., 2009). 


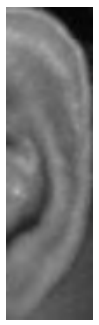

(a)

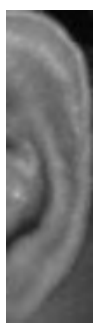

(b)

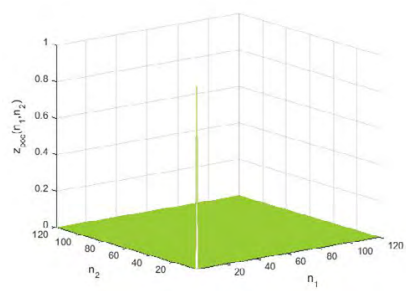

(c)

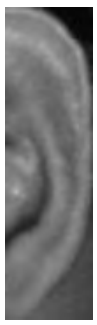

(d)

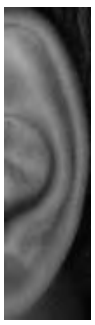

(e)

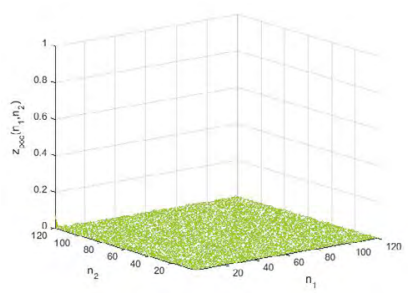

(f)

Figure 5. (a) Gallery image, (b) Query image, (c) POC for the same ear images, (d) Gallery image, (e) Query image and (f) POC for different ear images.

\subsection{BAND-LIMITED PHASE ONLY FUNCTION/CORRELATION (BLPOC)}

Consider to facilitate the range of the intrinsic frequency band be set through $a_{1}=-a_{1} \ldots$ $a_{1}$ and $a_{2}=-a_{2} \ldots a_{2}$, where $0 \leq a_{1} \leq M_{1}$ and $0 \leq a_{2} \leq M_{2}$. Therefore, the valuable size of frequency spectrum be set through $L_{1}=2 a_{1}+1$ and $L_{2}=2 a_{2}+1$ (Miyazawa et al., 2006). The BLPOG is known by means of

$$
r_{f g}^{a_{1}, a_{2}}\left(x_{1}, x_{2}\right) \frac{1}{L_{1} L_{2}} \sum_{a_{1}, a_{2}} \operatorname{Rg} f\left(a_{1}, a_{2}\right) W_{N 1}^{-a_{1} n_{1}} W_{N 2}^{-a_{2} n_{2}}
$$

Where $x_{1}=-a_{1} \ldots a_{1}, x_{2}=-a_{2} \ldots a_{2}$ and $\Sigma_{a_{1}, a_{2}}$ denote $\Sigma_{a=-a_{1}}^{a_{1}}$. Remind that the highest value of correlation peak of BLPOC is constantly normalized to 1 and it not lean on L1 and L2 (Nanni \& Lumini, 2007).

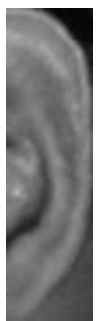

(a)

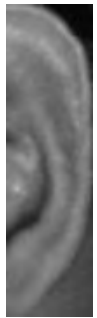

(b)

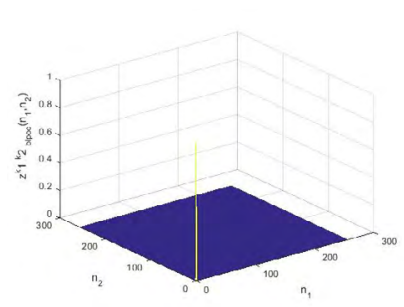

(c)

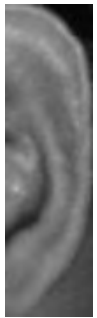

(d)

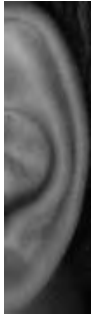

(e)

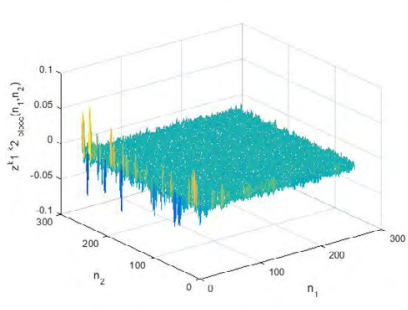

(f)

Figure 6. (a) Gallery image, (b) Query image, (c) BLPOC for the same ear images, (d) Gallery image, (e) Query image and (f) BLPOC for different ear images. 


\section{EXPERIMENT AND DISCUSSION}

The intended method be extensively investigated using an Ear images database provided by IIT Delhi. It is contained of the ear images, where collected from the students and staff at IIT Delhi (Zhu et al., 2009). In this paper proposed method are implemented and evaluated by using MATLAB software. Next in this paper proposed method has employee two processes: one is POC based phase matching components of two same images and two different images. Another one is to perform BLPOC based phase matching components of two same images and two different images. This issue has been showed in Figure 4 and 5 . The Figure 5 shows POC level estimated at 0.64, it express high matching trial has achieved. Uniformly Figure 6 shows BLPOC level estimated at 0.14, it express high matching trial has achieved. It shows better performance than other modalities that are previously existed.

\subsection{PERFORMANCE EVALUATION OF HUMAN EAR RECOGNITION}

The Receiver Operating Characteristic (ROC) curve evaluates the performance of the biometrics-based authentication system, which constructs with the help of False Reject Rate (FRR) and False Accept Rate (FAR) in various thresholds resting on matching score. Primarily, estimate the FRR in support of all the probable combinations number of authentic attempts. Similarly, estimate the FAR in support of all the probable combinations number of imposter attempts shown in Figure 7. In Figure 8, the ROC curve belongs to BLPOC; it demonstrates this algorithm is suitable for recognizing human ear images. Hence, proposed algorithm consider overall and confined deformation of human ear images together to calculate the scores of matching among the human ear images. Table 2 shows the EER [\%] and distance $\left(d^{\prime}\right)$ values of Ear identification.

$$
\mathbf{d}^{\prime}=\frac{\sqrt{2}\left|\mu_{\text {genuine }}-\mu_{\text {imposter }}\right|}{\sqrt{\sigma_{\text {genuine }}^{2}+\sigma_{\text {imposter }}^{2}}}
$$

Table 2. Equal error rate and distance (d') of the human ear recognition.

\begin{tabular}{|c|c|c|}
\hline Proposed algorithm & Equal error rate (\%) & Distance (d') \\
\hline BLPOC & 0.86 & 2.145 \\
\hline
\end{tabular}




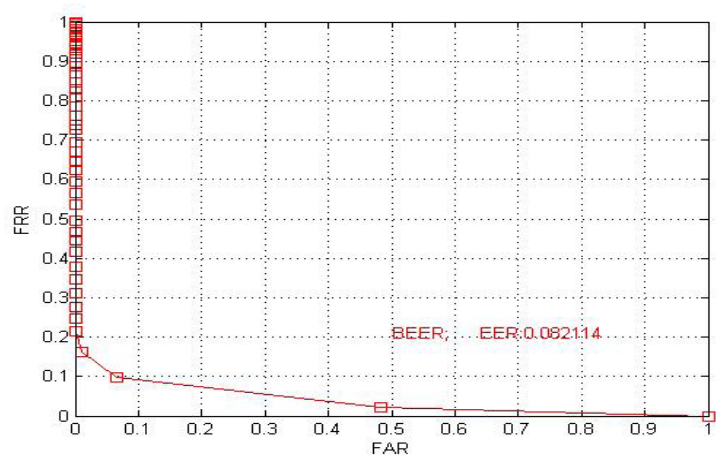

Figure 7. EER rate of proposed system.

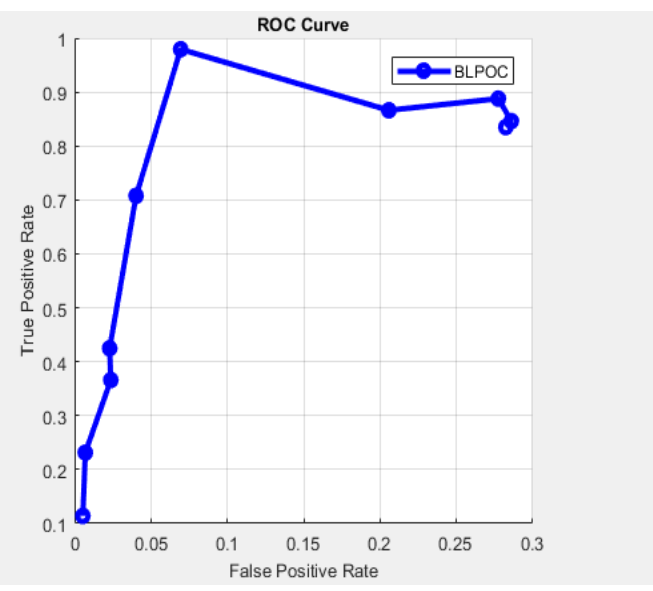

Figure 8. ROC curves for BLPOC algorithm.

Where, the mean value of genuine and imposter matching scores, respectively to an authentic and fraud identification, and the standard deviation of genuine and imposter matching scores, respectively to an authentic and fraud identification. The performances of a proposed method are able to assess in terms of identification accuracy, which is specified in the equation (17).

$$
\text { Accuracy }=100-\frac{\text { False rejection rate }+ \text { False acceptance rate }}{2}
$$




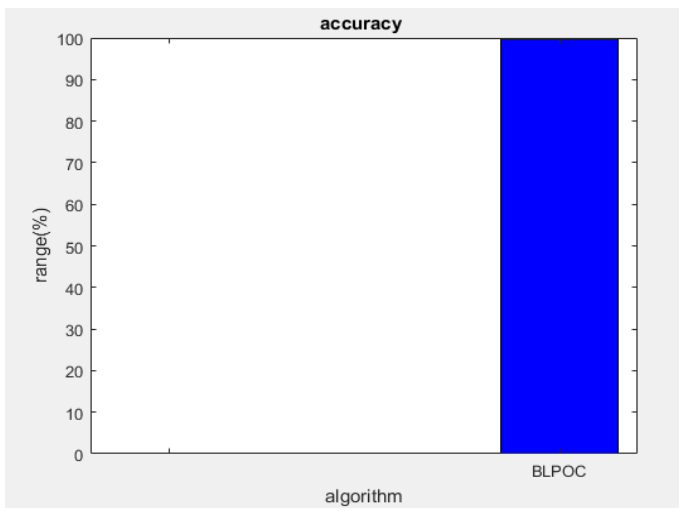

Figure 9. Accuracy of BLPOC algorithm.

The Figure 9 shows the accuracy of BLPOC algorithm. Where, FRR is the false rejection rate at which an authentic person is wrongly rejected as an imposter and FAR is the false acceptance rate, which denotes the rate of an imposter is wrongly accepted as an authentic person.

\section{CONCLUSION}

This paper intends a human ear identification using phase based function matching algorithm. The proposed BLPOC algorithm of Human Ear Recognition makes it possible to align ear images, correctly evaluated similarity between them and obtained the reliable matching score. The experimental results reveal that the intended method has achieved the well again Human Ear recognition performance and robustness over other previous methods. Extensively experiments were tested on the Ear database IIT Delhi. The proposed method of human ear recognition has performed the finest verification results on the Ear database IIT Delhi, with the equal error rate $0.86 \%$.

\section{ACKNOWLEDGEMENT}

I thank the department of Electronics and Communication Engineering of Kalasalingam University, (Kalasalingam Academy of Research and Education), Tamil Nadu, India for permitting to use the computational facilities available in Signal Processing and VLSI Design laboratory which was setup with the support of the Department of Science and Technology (DST). 


\section{REFERENCES}

Aoyama, S., Ito, K., \& Aoki, T. (2014). A finger-knuckle-print recognition algorithm using phase-based local block matching. Information Sciences, 268, 53-64. https://doi. org/10.1016/j.ins.2013.08.025

Bustard, J. D., \& Nixon, M. S. (2010). Toward unconstrained ear recognition from twodimensional images. IEEE transactions on systems, man, and cybernetics-Part A: Systems and Humans, 40(3), 486-494. https://doi.org/10.1109/TSMCA.2010.2041652

IIT Delhi Ear Database. (2007). www.iitdelhi/biometric/ear.html

Ito, K., Nakajima, H., Kobayashi, K., Aoki, T., \& Higuchi, T. (2004). A fingerprint matching algorithm using phase-only correlation. IEICE transactions on fundamentals of electronics, communications and computer sciences, 87(3), 682-691. http://www.aoki.ecei. tohoku.ac.jp/research/docs/e87-a_3_682.pdf

Jain, A.K., Ross, A., \& Pankanti, S. (2006). Biometrics: a tool for information security. IEEE transactions on information forensics and security, 1(2), 125-143. https://doi. org/10.1109/TIFS.2006.873653

Mahri, N., Suandi, S. A., \& Rosdi, B. A. (2010, August). Finger vein recognition algorithm using phase only correlation. In 2010 International Workshop on Emerging Techniques and Challenges for Hand-Based Biometrics (pp. 1-6). IEEE. https://doi.org/10.1109/ ETCHB.2010.5559283

Malathy, G., Annapurani, K., \& Sadiq, A. K. (2013). Analysis of fusion methods for ear biometrics. International fournal of Advanced Research in Computer Science and software Engineering, 3(1), 181-185.

Miyazawa, K., Ito, K., Aoki, T., Kobayashi, K., \& Katsumata, A. (2006, October). An iris recognition system using phase-based image matching. In 2006 International Conference on Image Processing (pp. 325-328). IEEE. https://doi.org/10.1109/ ICIP.2006.313159 
Miyazawa, K., Ito, K., Aoki, T., Kobayashi, K., \& Nakajima, H. (2005, September). An efficient iris recognition algorithm using phase-based image matching. In IEEE international conference on image processing 2005, (Vol. 2, pp. II-49). IEEE. doi: https://doi. org/10.1109/ICIP.2005.1529988

Nanni, L., \& Lumini, A. (2007). A multi-matcher for ear authentication. Pattern Recognition Letters, 28(16), 2219-2226. https://doi.org/10.1016/j.patrec.2007.07.004

Rutty, G. N., Abbas, A., \& Crossling, D. (2005). Could earprint identification be computerised? An illustrated proof of concept paper. International Journal of Legal Medicine, 119(6), 335-343. https://doi.org/10.1007/s00414-005-0527-y

Takita, K., Muquit, M. A., Aoki, T., \& Higuchi, T. (2004). A sub-pixel correspondence search technique for computer vision applications. IEICE transactions on fundamentals of electronics, communications and computer sciences, 87(8), 1913-1923. http://www.aoki.ecei. tohoku.ac.jp/research/docs/e87-a_8_1913.pdf

Yazdanpanah, A. P., \& Faez, K. (2010). Ear Recognition Using Bi-Orthogonal and Gabor Wavelet-Based Region Covariance Matrices. Applied Artificial Intelligence, 24(9), 863-879. https://doi.org/10.1080/08839514.2010.514228

Yuan, L., Wang, Z. H., \& Mu, Z. G. (2010, April). Ear recognition under partial occlusion based on neighborhood preserving embedding. In Biometric Technology for Human Identification VII (Vol. 7667, p. 766701). International Society for Optics and Photonics.

Zhang, L., Zhang, L., \& Zhang, D. (2009, September). Finger-knuckle-print verification based on band-limited phase-only correlation. In International Conference on Computer Analysis of Images and Patterns (pp. 141-148). Springer, Berlin, Heidelberg.

Zhang, L., Zhang, L., Zhang, D., \& Guo, Z. (2012). Phase congruency induced local features for finger-knuckle-print recognition. Pattern Recognition, 45(7), 2522-2531. https://doi.org/10.1016/j.patcog.2012.01.017

Zhang, L., Zhang, L., Zhang, D., \& Zhu, H. (2011). Ensemble of local and global information for finger-knuckle-print recognition. Pattern recognition, 44(9), 1990-1998. https://doi.org/10.1016/j.patcog.2010.06.007 
Zhu, Y. H., Jia, W., \& Liu, L. F. (2009, September). Palmprint recognition using bandlimited phase-only correlation and different representations. In International Conference on Intelligent Computing (pp. 270-277). Springer, Berlin, Heidelberg. 


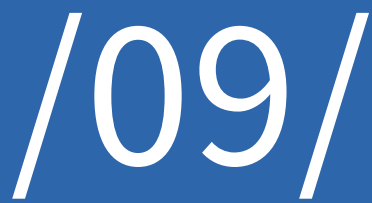




\title{
NEW INTUITION ON EAR AUTHENTICATION WITH GABOR FILTER USING FUZZY VAULT
}

\author{
A. Kavipriya \\ Department of Electronics and Communication Engineering, \\ Kalasalingam Academy of Research and Education, \\ Krishnankoil, (India).
}

E-mail: kavidivya222@gmail.com ORCID: https://orcid.org/0000-0002-2965-2542

M. Arunachalam

Department of Electronics and Communication Engineering,

Kalasalingam Academy of Research and Education,

Krishnankoil, (India).

E-mail: muthuece.eng@gmail.com ORCID: https://orcid.org/0000-0001-8070-3475

\section{Citación sugerida:}

Kavipriya, A., y Arunachalam, M. (2020). New Intuition on Ear Authentication with Gabor Filter Using Fuzzy Vault. 3C Tecnología. Glosas de innovación aplicadas a la pyme. Edición Especial, Marzo 2020, 159-179. http://doi.org/10.17993/3ctecno.2020.specialissue4.159-179

\section{Suggested citation:}

Kavipriya, A., \& Arunachalam, M. (2020). New Intuition on Ear Authentication with Gabor Filter Using Fuzzy Vault. 3C Tecnología. Glosas de innovación aplicadas a la pyme. Edición Especial, Marzo 2020, 159-179. http://doi.org/10.17993/3ctecno.2020.specialissue4.159-179 


\section{ABSTRACT}

At present, Frequent Biometrics Scientific Research deals with other biometric application like Face, Iris, Voice, Hand-Based Biometrics traits for classification and spotting out the persons. These Specific Biometric traits have their own improvement and weakness for opting the terms like Accuracy \& cost of all applications. However, in addition to other Face-based Biometric techniques, Ear Recognition has been appealed to Boom the attention among other Biometric researchers. This Image Template Pattern Formation of Ear cuddles the report which is relevant for maculating the Uniqueness of their individuality. This Ear Biometric trait observes the person's identity based on its stable Anatomical behavior. This biometric trait does not involve any emotional feelings with facial expressions in the same way as a unique pair of Fingerprint. In this work, a Contemporary approach for Personal identification is imported with Ear along with the data stores in a secured way has been proposed. This authentication Process includes the revolution of features with Gabor Filter and Dimension Reduction based on Multi-Manifold Discriminant Analysis (MMDA). This work is adequately analyzed in Matlab with the Evaluation metrics such as FMR, GAR, FNMR, by modifying the key value each time. The results of this suggested work promote better values in recognition of individuals as for Ear modalities. Conclusively the Features are grouped using K-Means for both identification and Verification Process. This Proposed system is initialized with Ear Recognition Template based on Fuzzy Vault. The Key stored in the Fuzzy Vault is utilized in safeguarding the existence of Chaff Points.

\section{KEYWORDS}

2D Gabor Filter, Multi-Manifold Discriminant Analysis (MMDA), K-Means, False Matching Rate (FMR), False Non Matching Rate (FNMR), Genuine Acceptance Rate (GAR). 


\section{INTRODUCTION}

In the present day scenario, the booming demand in case for both Security and automated recognition system leads to radical research resolution in the various areas of Computer Vision and Intelligent systems. At Present Periodic arrangements of individual identity happens through the enactment of Password with Permissive Activities in Public security, Access Control, Computer Vision as well as Intelligent Systems. Therefore Biometrics is considered as a significant application of forensics, Surveillance examination which assigns to the technique of diagnosing the Humans by utilizing their physical or behavioral traits along with faces, Iris, Fingerprint, Ear, Palm print, FKP, voice, and signature. These features can be treated as Biometric diagnostic features with satisfaction of requirements: (i)Universality, (ii)Distinctiveness, (iii)Permanence, (iv)Performance, (v)Collectability, (vi) Acceptability. Each of these above mentioned biometric procedure has both its precedence and nuisance using single modality which is optimal for other types of Professional systems applications. This paper targets on Human Ear as one of the auspicious and idiosyncratic biometric modality that involves enduring and dependent with a shape which does not expose desperate contradiction with age.

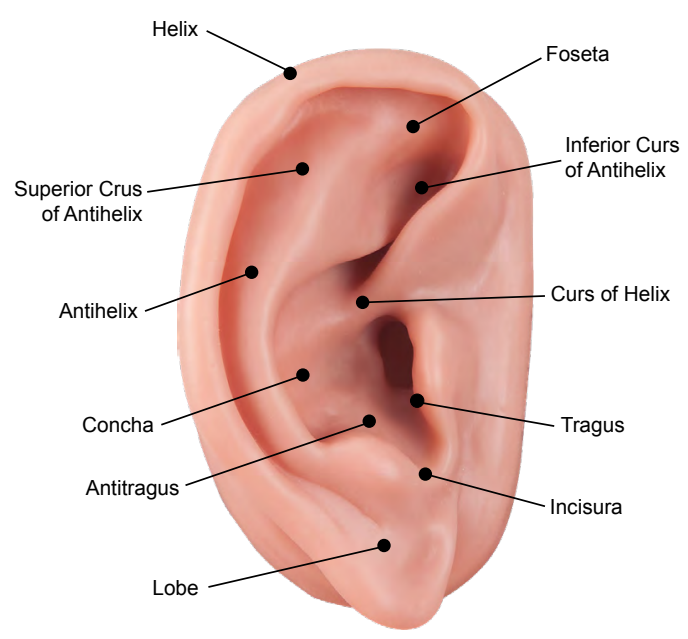

Figure 1. Anatomy of Human Ear.

Based on Figure 1 explains the External anatomical Structure of Human Ear with its lingual components including Helix, Antihelix, Tagus, Concha, Lobe and further Parts. This Infinite ridges and Valleys on the external Ear's Surface act as phonic Signals. In case 
of Low Frequencies, Pinna reproduces the acoustic signals near to the ear canal. Similarly, High Frequencies reverberate the acoustic wave which causes the frequencies to be calm down. This makes to note that origin of Sound perceived enabled by humans in Outer Ear. Random Factors of Ear's appearances can be first examined by correlating the Left and Right Ear of the similar Persons.

The ear Recognition can be approximately restricted based on the categories of several methods like Hassaballah, Alshazly, and Ali (2019) explains the EAR authentication complication utilizing Local Binary Patterns (LBP) Features. This Monotonic image of Gray-Scale factor transforms with its computational efficiency with Local Binary Pattern features that is fit for Ear Recognition problem. This tested LBP variants almost show the accuracy rate around 99\%, while the attainment faces several difficulties when the level of distortion boost. Likewise, Gandhimathi and Janarthanan (2019) describe the new class of Biometric as Ear recognition in comparison of Fingerprint that can be smoothly confiscated from the area's measures. Alike due to the emotion the shape of Ear does not change even due to the emotion. It is relatively constant over a Person's life. Robust Feature extraction helps in determining the personality of several individuals, for instance, terrorists at airports \& stations. Similarly, Gandhimathi and Radhamani (2016), and Gandhimathi and Janarthanan (2019), defines the effective fusion method for the combination of various data that can be secured by the generation of chaff points. These chaff points help in the formation of a secret key using the unimodal biometric data with feature vectors. The optimal location of these feature vectors is basically created by the fitness value as well as the development of security enhancement with the help of multi-biometric systems by means of the proposed modified template of Log Gabor Features XOR pattern. This kind of template security is basically determined by the other way of fuzzy vault multi- biometric cryptosystem. Vinothkanna and Amitabh (2014) explains the grouped feature vector resemblance points that are developed by chaff points and feature points. This grouped vector points in the fuzzy vault database lead to the accurate identification of the recognized individuals with correct match points. Several evaluation metrics like FAR, FRR, GAR, Secret key helps in the assessment of grouped vector points. Bansal and Hanmandlu (2017) presents the ear based identification function by the means of entropy values with reference to change in information gain information score values. This effective 
Gaussian and Exponential function find in generating the refined scores that are facilitated using the Euclidean distance metrics. Anwar, Ghany, and Elmahdy (2015) developed an advanced algorithm in case of Ear Recognition with geometric features extraction. Here Ear recognition with geometric features extraction. The ear detection generally based on the snake model with a median filter for removal of noises. Then canny edge and distance metrics are created with these image features. This method is invariant to translation\& rotation with accuracy of 98\%. Kacar and Kirci (2018) introduced the novel architecture with Score Net in case of Ear recognition. This method creates with modality pool in accordance with cascade fusion learning that is compatible with parallel processing. Lakshman (2013) implemented the double-stage geometric approach in both scale features $\&$ rotation invariant in case of uprooting the unique features. Hence the matching scores are compared with the basics of threshold values with authenticating the persons. Herewith PSO technique helps in Optimize the parameters with threshold and weights which helps in regulating the computation time. Rathgeb, Pflug, Wagner, and Busch (2016) deals with the image compression that helps in ear recognition stages with stimulated image distortions and partitions. Finally the detailed investigation of image compression technique. The feature extraction was calculated with uncompressed samples of Ear databases with numerous bitrates. Pflug and Busch (2012) discovered the identification by Ear recognition in case of 2D, 3D images in case of smart surveillance \& forensic image analysis. It explains the database collection with various features against various techniques. Nandakumar, Jain, and Pankanti (2007) extracts the highest curvature points that are helpful in aligning the template. Minutiae Matcher of decoding part leads to non-linear distortion which gives a significant improvement of GAR. Koptyra and Ogiela (2015) present a unique idea of hiding the secrets using the fuzzy vault. It is mainly hidden the noisy data based on multibiometric cryptosystem. It proposes a choice of authentication accuracy relevant with a cryptosystem on single biometric. Bae, Noh, and Kim (2003) shows the encoding of iris code that helps in the performance of EER that gives the magnitude performance for iris size along with processing time. Arunachalam and Kanan (2015) integrate the secret key value using Advanced Encryption Standard to avoid several attacks like spoofing, intraclass variations, etc., for the generation of biometric key utilizing the cryptographic fusion Uludag and Jain (2006) aims in the safeguarding and aloofness of biometric systems with the 
transformed version of the template that is stored as a cryptographic framework. So they introduce the orientation field of helper data for the extraction of fingerprints. Yang, Sun, and Zhang (2011) proposed the dimensionality reduction method for pattern recognition purposes that is based on graph embedded learning. This technique mainly based on the construction of low dimensional data. Basically, it cannot apply for small size problem. To overcome this, MMDA is calculated for Eigenvectors and Eigen value representations. Yang, Sun, and Wang (2011) have attracted interest against Gabor feature with MMDA. Certain Fuzzy vault system is generally on the support of local iris feature points from the exact values of an unordered set with basis if shift matching technique.

Remaining paper is formulated as follows: Section $2 \& 3$ precedes the inquiry of scheduled work and it portrayed the Fuzzy Vault which includes eradication of Ear along with Gabor features and grouped according to k-Means clustering algorithm in a detailed manner and Section 4 provides the particulars in relation to the basic Fuzzy Vault construction with enrolment and verification phase. Experimental decision is essentially explained in Section 5 and finally $6^{\text {th }}$ section entirely organizes the basic work that provides points for future research. The motivation of this work involves the Human Authentication that must be considered as the most important tasks which are used in this world for the case of identification of Persons using biometrics with its Physical and Behavioral Characteristics. They include Fingerprints, Handprints, Palm prints, Hand veins, Eyes, Ears, Voice, and signature. Basically, this biometric system is categorized as Unimodal; Multimodal, and Multi-biometric system, etc. This unimodal biometric System has severe challenges against noisy data. In this work, the Ear modalities are selected to generate the polynomial construction to the let the secure key in a collapsed manner. The reason for selecting the ear as main modalities is due to surprising rich features with it. Changes do not happen due to its stable structure.

\section{SYNOPSIS OF THE PROPOSED WORK}

This proposed paper suggested the flow diagram that explains briefly about the Ear recognition with the generation of polynomial construction in both locking and unlocking set for the case of Fuzzy Vault system. This work illustrates the cryptographic fuzzy vault technique as three phases. In Recognition phase the Ear images are collected from the 
database for the further process of dimensionality reduction phase. Further the enrollment phase the Gabor feature extraction is carried with five scales and eight orientations. These extracted features basically have high dimensional values. Minimization of this idea is accessed by the MMDA Technique in the calculation of Eigen value and Eigenvector that are explained below in this block Diagram.

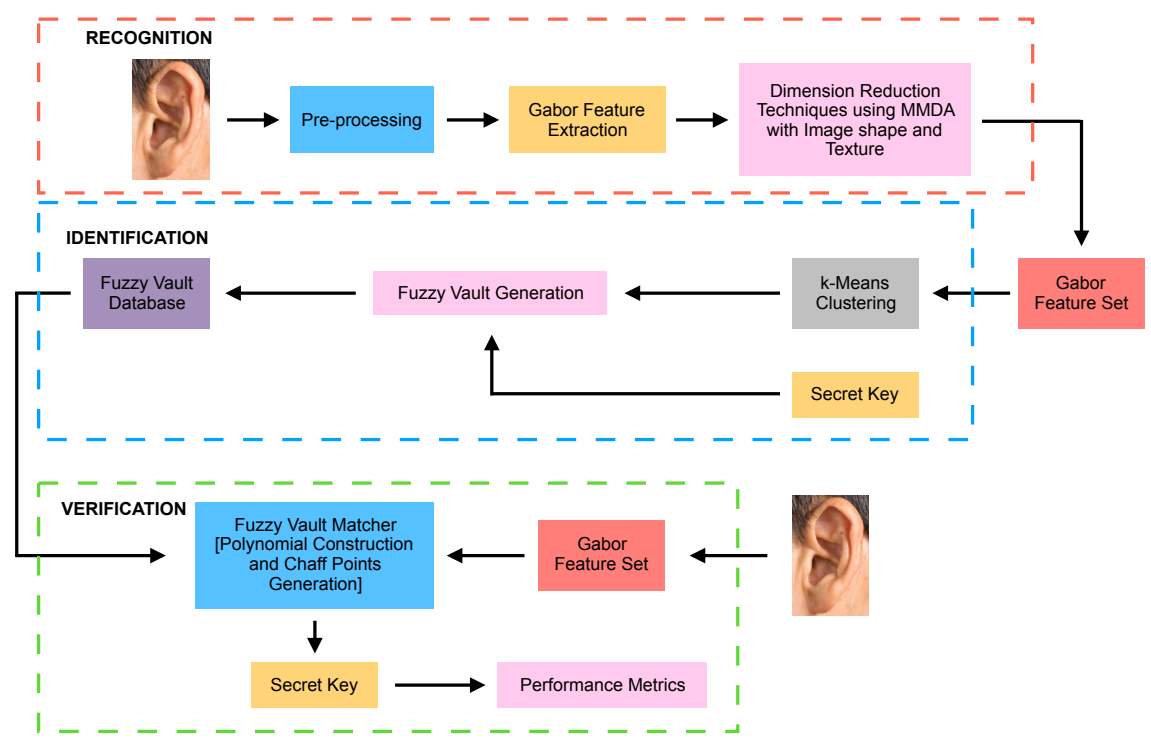

Figure 2. Flow diagram of proposed work with Fuzzy Vault Technique.

This Figure 2 shows the process of Ear recognition with several methods that include based on Fuzzy Vault. This vault helps in providing the security to several biometric cryptosystems. Here the chaff points are formed promptly from the biometric features which are identified easily. The features are clustered based on manifold learning Process. The origination of chaff points or secret key by the process of the vault locking process. Locking process creates the Polynomial generation of chaff points as a key that must be entered. Similarly, the Testing phase the same procedure is repeated in order to assess the common features and matching is done based on the revealing of the secret key along with biometrics. There are four significant stages in this proposed work:

\section{A. Pre-processing.}

\section{B. Gabor Feature Extraction.}




\section{Generation of Polynomial construction of grouped feature vectors.}

\section{Identification and Authentication of Secret Key.}

\section{A. Pre-Processing Phase}

Since the early phase, the images are to be pre-processed along with the objective of getting rid of the rejected part in image such as noise, Blur, reflections. Originally, these Ear images are reformed into gray scale images in the datasets are in RGB format. Thus the training process is enforced with ear datasets. Specific basic non-linear methods recycled are the median Filter. The main method of this filter helps in glaring of edges that helps in reducing the noises with the point of subsiding the current pixel point with the median of illumination in its range.

This center pixel appraisal is named as "median" and similarly the neighboring pattern as "window".

$$
H(m, n)=\operatorname{median}[x(m-k, n-l) \hat{I} w]
$$

This equation 1 " $w$ " imitates the window along with the pixels $(m, n)$. Here the inured input images of the ear are expertly pre-processed and represented as $I_{e}$. Further this images separable which is cropped out to obtain the ROI with the help of changing the image size and Pixels.

\section{B. Gabor Feature Extraction\& k-means clustering}

Gabor feature Extraction is based on spatial locality and oriented selectivity with Ear Images. Gabor wavelets formation is developed. Gabor wavelet formation is developed with the kernels which are to identical to certain profiles and exposing the desirable location and orientation selectivity. This Gabor wavelet determination is to be entitled as:

$$
\left.\psi_{u, v(z)}=\left\|\frac{k_{u, v}}{\sigma_{2}}\right\|^{2} e^{\left(-\left|\frac{k(u, v)}{2 \sigma^{2}}\right|^{z}\right.}\right)\left(e^{i k_{u, v}} e^{\frac{-\sigma^{2}}{2}}\right)
$$

Where $\mathrm{u}, \mathrm{v}$ denotes the direction, scales of Gabor feature kernels. It is defined based on norm operator 
Where:

$$
k_{u, v}=\frac{k \max }{\frac{v}{2}}
$$

This factor "K max" represents high frequency and $\mathrm{f}$ depicts spacing vector with five scales and eight orientations. Further convolution of Gabor features is based on $Z(x, y)$ that serves the ultimate position of the figure and *denotes convolution operator.

\section{Multi-Manifold Discriminant Analysis (MMDA)}

Collection of Sample set with various ear data label is denoted as

$$
A=\left[A_{1} A_{2}, A_{3} \ldots A_{N}\right] A_{i} \sum R_{M}
$$

Likewise the linear projection of low dimensional space is defined as the

$$
B=P^{T} A^{r}
$$

Considering the points with several similar class labels that Possess edge construction between the nodes $y_{i}, y_{j}$ from the corresponding class. It is also broadly promoted such as $y_{i}, y_{j}$ with its parameter

$$
o \leq s_{i j} \leq 1
$$

Here weight functions are taken as an important note with strict monotonically decreasing function. Apparently, it has been noticed with negative non-symmetric that are exalted by the matrices between Class and within class scatter in $\beta_{W}, \beta_{B}$

$$
J(P)=\arg _{p} \max \equiv \frac{J_{R}(P)}{J_{W}(P)}
$$

Therefore it can be represented as:

$$
\frac{P^{T} B_{b} P}{P^{T} B_{W} P}
$$


Hence the projection matrix is generally represented as:

$$
\beta_{b} p=\lambda \beta_{w} p
$$

This projection matrix is literally named after the Graph embedding algorithm which is intended by the Eigen Value. These processes are clustered by the part of k-means clustering by calculating the centroid points and accredited these points towards the center.

\section{Glustering using K-means}

Clustering mainly used to acclimate the feature points based on the performance of unsupervised classification of certain patterns as groups. Considering the size of input and classification in large groups, $\mathrm{k}$-means clustering target the execution process with a basis of Ear feature points. Further, it is continued based on centroid calculation. Basically, it is like the expectation-maximization algorithm with mixtures of Gaussian in the process of finding clustering with various attributes.

$$
J=\sum_{j=1}^{k} \sum_{i=1}^{n}\left\|x_{i}^{j}-c_{1}^{2}\right\|
$$

Where J represents the objective function that is to be defined number of cases and centroid for cluster points that are based on Euclidean distance with distance measure defined as the classification of objects.

\section{Algorithm:}

Input: $\mathrm{k}$ and other points with $\mathrm{b}_{1}, \mathrm{~b} 2 \ldots \ldots \mathrm{b}$; Clustering the data into several $\mathrm{k}$ groups.

Gluster Update: Selecting k points at random cluster Centers.

Centers Update: Assigning articles to the adjoining cluster Centre to determine according to the Euclidean distance.

Stopping Update: Determining the centroid points or mean of severalEar featuresin ever Cluster.

Output: Repetition of steps 2, 3 until similar points assigned to each cluster. 


\section{c. Generation of Polynomial construction of grouped feature vectors}

In order to assigning the template security, Secret key plays the main role in generating the fuzzy vault that is united to form grouped feature vector. Originally the intake of secret keyis concealed with the number of chaff point's generation. Considering the information stored in the dataset is Permanent, Security is taken as an important note. Fuzzy vault is radically a cryptographic construction recommended by Juels and Sudan (2006) securing the critical data with the help of biometric data.

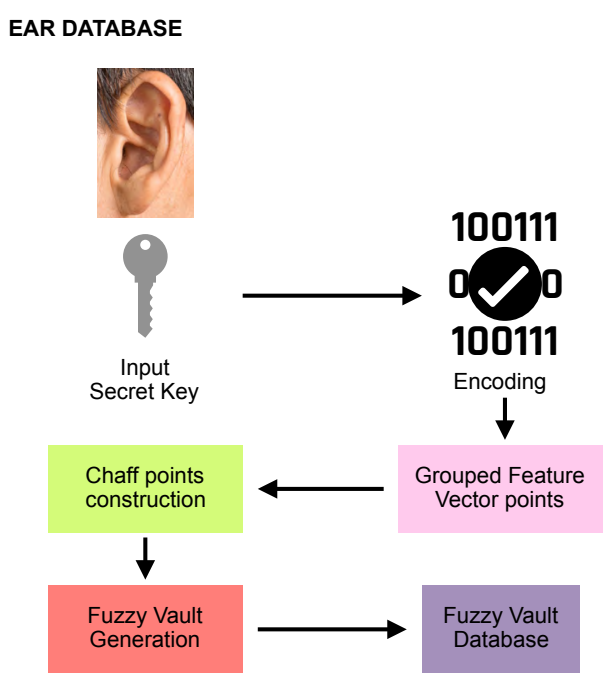

Figure 3. Block diagram of Fuzzy vault Construct.

Based on Figure 3 Polynomial construction with genuine points are stored as a secret key from the Ear database. Usually, the secret key information that is distributed as unordered sets named as Chaff points. These chaff points basically denote the content of secure information to be reconstructed for revealing the secret code which is stored in the Fuzzy vault database.

\section{d. Identification and Authentication of Secret Key}

In the recognition phase, Person's ear images are taken as input that is pre-processed and the features are extracted for the combination of feature vector. This input feature vector is compared to the fuzzy vault database. Matching relates with the secret key generation and authentication is proved. This recognition process is adorned. Let the given person's 
Gabor feature vector that must express by $c$ that is related to the fuzzy vault in the dataset. In case if every feature points of the ear image matches the features in the fuzzy vault, then the individual is admitted authentication or else the authentication is contradicted. Assured points in the fuzzy vault will be left deserted. These points are named as "secret points" and the x-coordinates of these features' points provide the secret key of the authenticated person.

Finally the procreation of the authenticated person is the second confirmation of the person which boosts the template security.

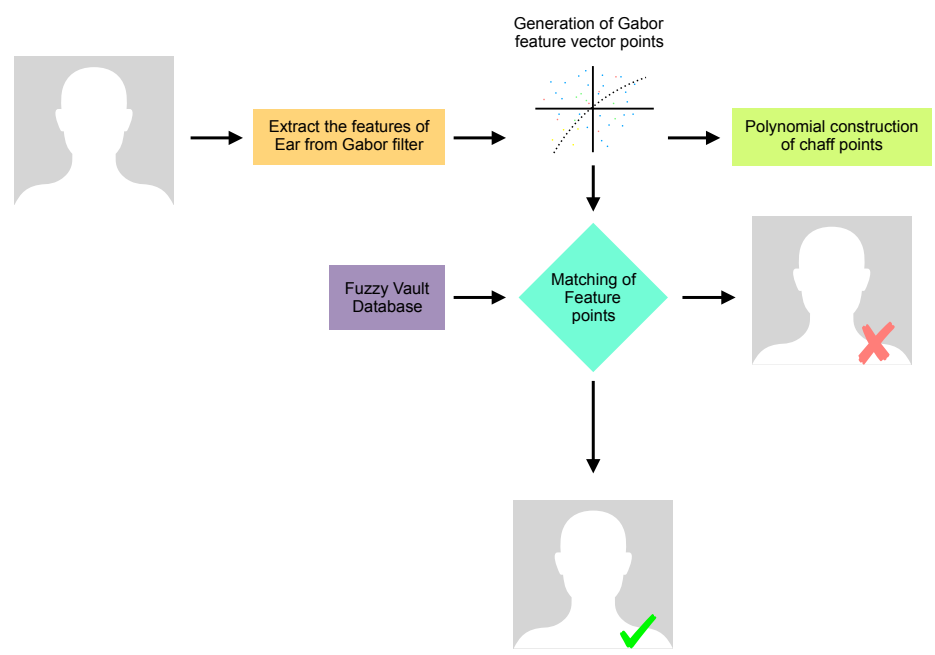

Figure 4. Recognition of a person with Fuzzy vault using Ear.

This Figure 4 represents the recognition of the person based on the person's ear image with Fuzzy vault. These features which are extracted from the Gabor filters are generated to form the polynomial construction of chaff points. These matched feature points are determined from the vault database gives the authentication of the person.

\section{EXPERIMENTAL RESULTS AND PERFORMANCE EVALUATION}

In this category, the consequences of the designed biometric method for the recognition of Ear modalities utilizing Fuzzy Vault are contended with detailed manner in this work. This Intended methodology is executed in Matlab Platform of version 2017. Dataset confession of IIT Delhi Ear images is utilized work. 


\section{EAR-IIT Delhi}

This dataset version 1.0 mainly incorporates the ear Images collected from the graduates and Faculties at IIT Delhi, India. This entire dataset in the dataset is chiefly around ages grouped under $14-58$ years. The directory of 471 images is progressively counted for every user with unique identification number. The intention of these figures is about $272 \times 204$ pixels and reachable in jpeg format. This dataset endeavors the naturally normalized and cropped ear images of size about $50 \times 180$ pixels beside the authentic images. A further large adaption of ear dataset from 212 users with 754 ear images is incorporated.
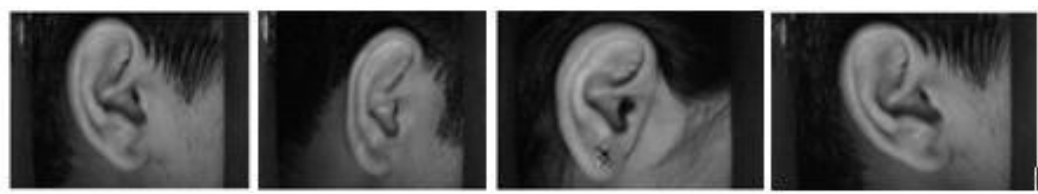

Figure 5. Illustration of Ear images from IIT Delhi Ear database Version 1.0.

\section{Experimental Results}

Originally these Ear figures are in gray scale format, it is very much accessible for filtering process. This filtering method includes Sobel filter which excludes the noise regions like thin hair, studs etc., and the Pre-processed process these figures are shown in the Figure 6.

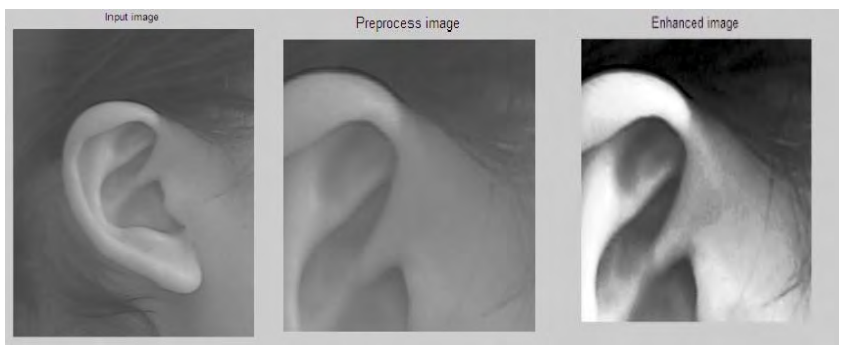

Figure 6. Results of Ear model (a) Input Figure (b) Preprocess Figure (c) Enhanced Image.

This Figure 6 shows the basic pre-processing and enhancement process which helps the enhanced image after histogram equalization that further moves to feature extraction of Gabor Filter. 


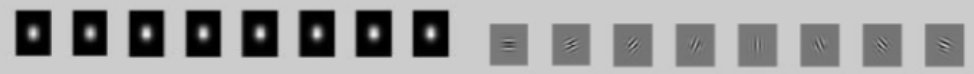

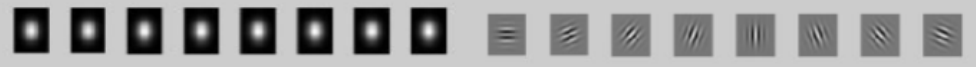

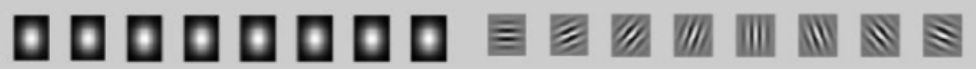

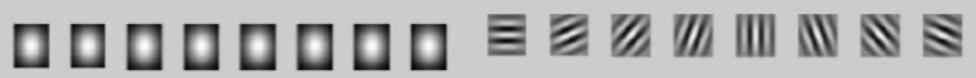

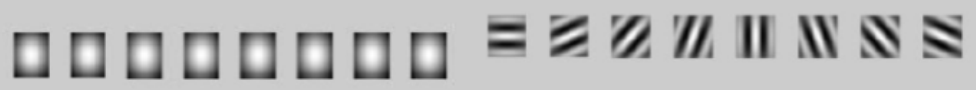

Figure 7. Magnitude and Real Parts of Gabor Filter.

The Figure 7 explains the Magnitude as well as Real Parts of Gabor feature Extraction which is determined from the Gabor Feature Extraction.

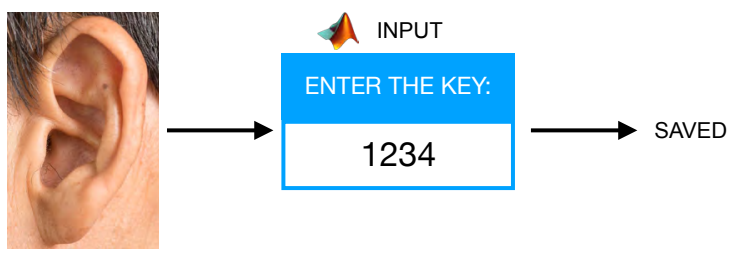

Figure 8. Encoding Process of Fuzzy Vault.

This Figure 8 explains the input image which are grouped as feature ve0ctors has been stored as [1234] in the Fuzzy Vault Database.

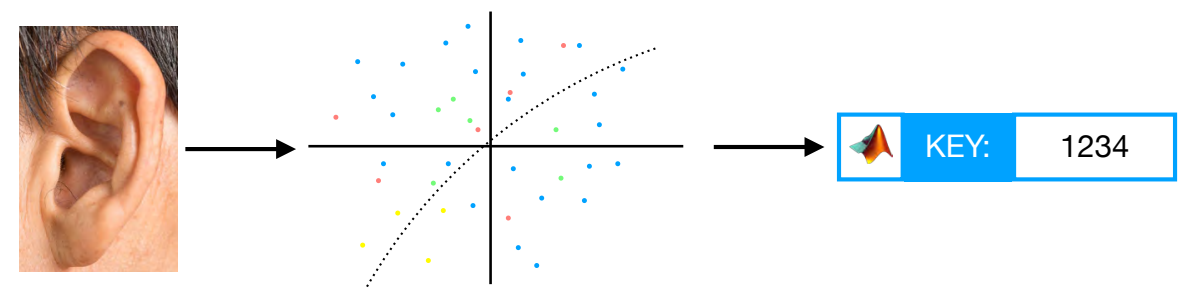

Figure 9. Decoding Process of Fuzzy Vault.

This Figure 9 shows the retrieval of Secret key from the Fuzzy Vault Database. It involves the grouped feature points that are indulged as chaff point's generation.

\section{Performance Evaluation Metrics}


To estimate this proposed biometric system it is established on Ear Images, several evaluation metrics are employed. This evaluation Metrics for this work involved are False Matching Rate (FMR), False Non-matching Rate (FNMR), Genuine Acceptance Rate (GAR) and Accuracy.

\section{False Matching Rate (FMR)}

This Matching Rate is based on the improper recognition of un-authorized People. The FMR is specified as resulted,

FMR = Number of un-authorized inputs with improper recognition

Total Number of Inputs

\section{False Non Matching Rate (FNMR)}

This Matching Rate is defined based on the improper recognition of authorized people. It has been defined as designed

FNMR $=$ Number of authorized Inputs that are falsely not recognized

Total Number of inputs

\section{Genuine Acceptance Rate (GAR)}

It is defined as the Probable of truly matching figures that are matched by the biometric security system with the entire images in the dataset.

\section{GAR=1-FNMR}

\section{Performance Analysis of this Proposed Work}

The results of this proposed image from Ear modalities are collected from 25 samples from various kinds of dataset. The results are taken based on the calculation of these evaluation metrics that is explained in Table 1.

Table 1. Analysis of biometric system with Ear modality.

\begin{tabular}{|c|c|c|c|c|}
\hline SI No. & FMR (\%) & FNMR (\%) & GAR (\%) & Accuracy (\%) \\
\hline 1 & 0.62 & 0.40 & 0.60 & 97.0 \\
\hline 2 & 0.60 & 0.40 & 0.61 & 98.0 \\
\hline 3 & 0.63 & 0.40 & 0.62 & 98.8 \\
\hline
\end{tabular}




\begin{tabular}{|c|c|c|c|c|}
\hline SI No. & FMR (\%) & FNMR (\%) & GAR (\%) & Accuracy (\%) \\
\hline 4 & 0.64 & 0.40 & 0.60 & 98.8 \\
\hline 5 & 0.72 & 0.30 & 0.70 & 96.0 \\
\hline 6 & 0.71 & 0.30 & 0.70 & 95.0 \\
\hline 7 & 0.70 & 0.30 & 0.70 & 98.1 \\
\hline 8 & 0.70 & 0.30 & 0.71 & 98.2 \\
\hline 9 & 0.80 & 0.20 & 0.80 & 96.0 \\
\hline 10 & 0.90 & 0.10 & 0.90 & 94.1 \\
\hline 11 & 0.70 & 0.30 & 0.70 & 98.8 \\
\hline 12 & 0.90 & 0.10 & 0.70 & 95.5 \\
\hline 13 & 0.60 & 0.40 & 0.60 & 94.1 \\
\hline 14 & 0.50 & 0.50 & 0.50 & 98.2 \\
\hline 15 & 0.70 & 0.30 & 0.70 & 98.8 \\
\hline 16 & 0.80 & 0.20 & 0.80 & 98.3 \\
\hline 17 & 0.60 & 0.40 & 0.60 & 94.0 \\
\hline 18 & 0.60 & 0.40 & 0.60 & 94.2 \\
\hline 19 & 0.70 & 0.30 & 0.70 & 95.5 \\
\hline 20 & 0.50 & 0.50 & 0.50 & 96.1 \\
\hline 21 & 0.40 & 0.60 & 0.40 & 97.1 \\
\hline 22 & 0.70 & 0.30 & 0.70 & 98.1 \\
\hline 23 & 0.80 & 0.20 & 0.80 & 98.9 \\
\hline 24 & 0.90 & 0.10 & 0.90 & 98.3 \\
\hline 25 & 0.50 & 0.50 & 0.50 & 98.4 \\
\hline
\end{tabular}

Table 1 shows the performance metrics of Ear modalities biometric system with various rates.

\section{CONCLUSION}

The stages in this work for this useful biometric system includes are (i) Pre-processing (ii) Gabor Feature Extraction (iii) Polynomial Construction of grouped vector from chaff points(iv)Identification and Authentication of secret key. These Proposed work biometric authentication systems with ear modalities are efficiently implemented in Matlab. Evaluation Metrics (FMR, FNMR, GAR) are calculated by frequently altering the key value. The analysis of this proposed work smoothened with better accuracy value as such $98.83 \%$. Further this idea will involve with multimodal biometric system to check its accuracy. 


\section{ACKNOWLEDGMENT}

We would like to be grateful for the International Research Centre of Kalasalingam Academy of Research and Education for investing financial assistance upon the scheme of University Research Fellowship (URF) and we also endorsed the Department of Electronics and Communication Engineering of Kalasalingam Academy of Research and Education, Tamil Nadu, India for providing usage of the computational facilities available in Signal Processing and VLSI Design laboratory that were set up with the assistance of the Department of Science and Technology (DST). 


\section{REFERENCES}

Anwar, A. S., Ghany, K. K. A., \& Elmahdy, H. (2015). Human ear recognition using geometrical features extraction. Procedia Computer Science, 65, 529-537. https://doi. org/10.1016/j.procs.2015.09.126

Arunachalam, M., \& Kanan, S. (2015). AES Based Multimodal Biometric Authentication using Cryptographic Level Fusion with Fingerprint and Finger Knuckle Print. The International Arab Fournal of Information Technology, 12(5), 431-440. https://www. semanticscholar.org/paper/AES-Based-Multimodal-Biometric-Authenticationusing-Arunachalam-Kannan/32a5f4d1d2f4c1d6ac521a486a7e20bc5c7b7da9

Bae, K., Noh, S., \& Kim, J. (2003). Iris feature extraction using independent component analysis. International Conference on Audio-and Video-Based Biometric Person Authentication. Springer, Berlin, Heidelberg. https://doi.org/10.1007/3-540-44887-X_97

Bansal, M., \& Hanmandlu, M. (2017). A new entropy function for feature extraction with the refined scores as a classifier for the unconstrained ear verification. Fournal of Electrical Systems and Information Technology, 4(1), 135-158. https://doi.org/10.1016/j. jesit.2016.10.006

Gandhimathi, A., \& Janarthanan, G. R. (2019). A Fuzzy vault based Multimodal biometric cryptosystem for enhancing security. International Fournal of Electrical and Computer Engineering, 768-773.

Gandhimathi, A., \& Radhamani, G. (2016). New chaff point based fuzzy vault for multimodal biometric cryptosystem using particle swarm optimization. Fournal of King Saud University-Computer and Information Sciences, 28, 381-394. https://core.ac.uk/ download/pdf/82045848.pdf

Hassaballah, M., Alshazly, H., \& Ali, A. A. (2019). Ear recognition using local binary patterns: A comparative experimental study. Expert Systems with Applications, 118, 182200. https://doi.org/10.1016/j.eswa.2018.10.007 
Hurley, D. J., Arbab-Zavar, B., \& Nixon, M. S. (2008). The ear as a biometric. In Jain A. K., Flynn P., Ross A. A. (eds) Handbook of Biometrics. Springer, Boston, MA, 131- 150. https://doi.org/10.1007/978-0-387-71041-9_7

Juels, A., \& Sudan, M. (2006). A fuzzy vault scheme. Designs, Codes and Cryptography, 38(2), 237-257.

Kacar, U., \& Kirci, M. (2018). ScoreNet: deep cascade score level fusion for unconstrained ear recognition. IET Biometrics, 8(2), 109-120. https://doi.org/10.1049/ietbmt.2018.5065

Koptyra, K., \& Ogiela, M. R. (2015). Fuzzy vault schemes in multi-secret digital steganography. In 2015 10th International Conference on Broadband and Wireless Computing, Communication and Applications (BWCCA). IEEE. https://doi.org/10.1109/ BWCAA.2015.87

Lakshmanan, L. (2013). Efficient person authentication based on multi-level fusion of ear scores. IET biometrics, 2(3), 97-106. https://doi.org/10.1049/iet-bmt.2012.0049

Lee, Y.J., Park, K. R., Lee, S. J., Bae, K., \& Kim, J. (2008). A new method for generating an invariant iris private key based on the fuzzy vault system. IEEE Transactions on Systems, Man, and Cybernetics, Part B (Cybernetics), 38(5), 1302- 1313. https://doi. org/10.1109/TSMCB.2008.927261

Nandakumar, K., Jain, A.K., \& Pankanti, S. (2007). Fingerprint-based fuzzy vault: Implementation and performance. IEEE transactions on information forensics and security, 2(4), 744-757. https://doi.org/10.1109/TIFS.2007.908165

Pflug, A., \& Busch, C. (2012). Ear biometrics: a survey of detection, feature extraction and recognition methods. IET biometrics, 1(2), 114-129. https://doi.org/10.1049/ietbmt.2011.0003

Rathgeb, C., Pflug, A., Wagner, J., \& Busch, G. (2016). Effects of image compression on ear biometrics. IET Biometrics, 5(3), 252-261. https://doi.org/10.1049/ietbmt.2015.0098 
Uludag, U., \& Jain, A. (2006). Securing fingerprint template: Fuzzy vault with helper data. In 2006 Conference on Computer Vision and Pattern Recognition Workshop (CVPRW'06). IEEE. https://doi.org/10.1109/CVPRW.2006.185

Vinothkanna, R., \& Amitabh, W. (2014). Fuzzy vault fusion based multimodal biometric human recognition system with fingerprint and ear. Fournal of theoretical and applied information technology, 59(2), 304-317. http://www.jatit.org/volumes/ Vol59No2/9Vol59No2.pdf

Yang, W., Sun, C., \& Wang, Z. (2011). Finger-knuckle-print recognition using Gabor feature and MMDA. Frontiers of Electrical and Electronic Engineering in China, 6(2), 374. https://doi.org/10.1007/s1 1460-011-0141-3

Yang, W., Sun, G., \& Zhang, L. (2011). A multi-manifold Discriminant analysis method for image feature extraction. Pattern Recognition, 44(8), 1649-1657. https://doi. org/10.1016/j.patcog.2011.01.019 


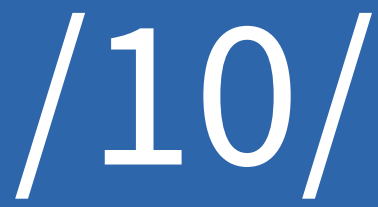




\title{
MEMETIC ALGORITHM BASED ON HILL CLIMBING ALGORITHM FOR IC PARTITIONING
}

\author{
K. Jeya Prakash \\ Assistant Professor, ECE Department, \\ Kalasalingam Academy of Research and Education \\ (Deemed to be University). \\ Krishnankoil, (India). \\ E-mail: k.jeyaprakash@klu.ac.in ORCID: https://orcid.org/0000-0001-7493-1914 \\ P. Sivakumar \\ Professor, ECE Department, \\ Kalasalingam Academy of Research and Education \\ (Deemed to be University). \\ Krishnankoil, (India). \\ E-mail: siva@klu.ac.in ORCID: https://orcid.org/0000-0003-1328-8093
}

Recepción: 05/12/2019 Aceptación: 17/12/2019 Publicación: 23/03/2020

\section{Citación sugerida:}

Jeya Prakash, K., y Sivakumar, P. (2020). Mememtic algorithm based on hill climbing algorithm for IC partitioning. 3C Tecnología. Glosas de innovación aplicadas a la pyme. Edición Especial, Marzo 2020, 181-193. http://doi.org/10.17993/3ctecno.2020.specialissue4.181-193

\section{Suggested citation:}

Jeya Prakash, K., \& Sivakumar, P. (2020). Mememtic algorithm based on hill climbing algorithm for IC partitioning. 3C Tecnología. Glosas de innovación aplicadas a la pyme. Edición Especial, Marzo 2020, 181 193. http://doi.org/10.17993/3ctecno.2020.specialissue4.181-193 


\section{ABSTRACT}

To reduce the premature convergence of the optimization problem, the genetic algorithm with local search called "memetic algorithm" is introduced. The proposed memetic algorithm can partition a complex circuit design into a few sub-circuits. The aim of this paper is to reduce the interconnects between the partitioned blocks. The experimental results show that the method is effective for solving the given input and to find the minimum cut size. Applying memetic algorithm like Hill Climbing algorithm for 3D IC partitioning is the novelty in this work.

\section{KEYWORDS}

Memetic algorithm, Genetic algorithm, Gircuit partition, Cut size. 


\section{INTRODUCTION}

Very-large-scale integration (VLSI) is a process which integrates many transistors into a single chip called "Integrated Circuit". An electronic circuit requires many sub circuits like CPU, ROM, RAM and other glue logic. VLSI made it possible to include all of them into one chip. Designers depend on Computer Aided Design (CAD) tools to provide a higher level of idea to reduce the complexity of circuits.

The phrase related with the mission of automatically designing a circuit by means of CAD tools is known as Electronic Design Automation (EDA). In VLSI design, physical design is one of the steps in the standard design cycle which trails the circuit design as shown in Figure 1. At this step, circuit representation of the devices and interconnects of the design are changed into geometric representations of shapes which, at the point when produced in the relating layers of materials, will guarantee the essential functioning of the components. This geometric representation is called IC layout.

Circuit partitioning is a vital step which ensures the interactions between circuit blocks is minimal. The minimal inter-partition communication may lead to have a few numbers of wires between them. This in turn leads to small interconnect delay and low power.
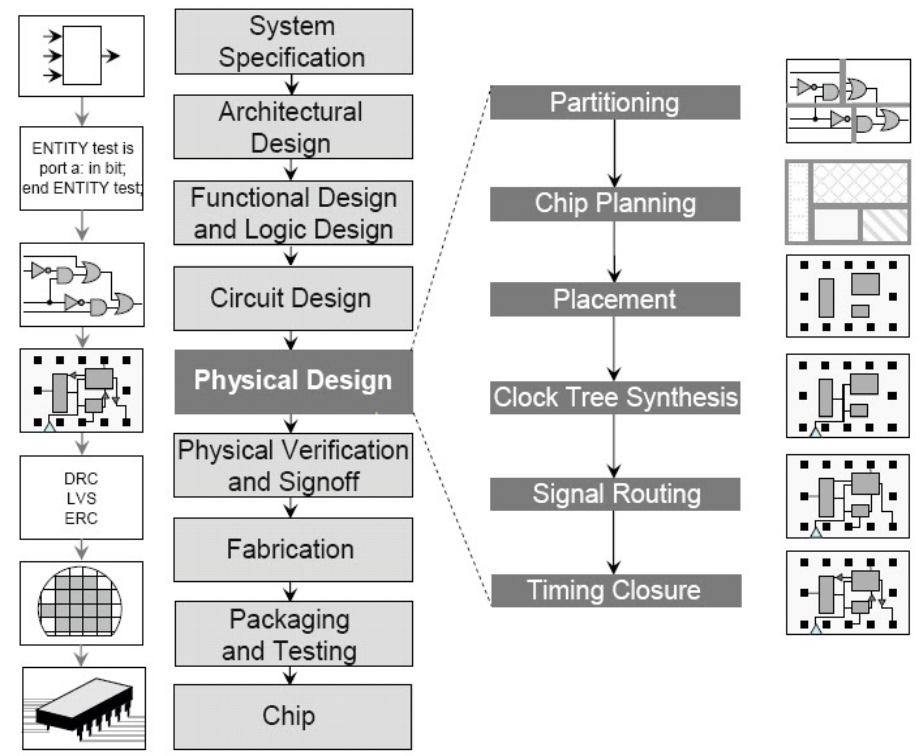

Figure 1. Design flow of VLSI. 
Hence, the main goal is partitioning a circuit into multiple blocks with an attempt to lessen the cut-size.

\section{PROBLEM FORMULATION}

In circuit partitioning problem, the logic representation of the circuit, modules and interconnection between modules are represented as geometric representation, vertices $(\mathrm{V})$ and edges $(\mathrm{E})$ of a graph $(\mathrm{G})$ respectively. The vertices and edges of $\mathrm{G}$ may be weighted to reveal module area or significance of an interconnection. The circuit partitioning has the following goals to make the IC compact:

Minimum Gut: Given $G=(V, E)$, partition $\mathrm{V}$ into disjoint subsets $\mathrm{X}$ and $\mathrm{Y}$ such that e $(\mathrm{X}$, $Y)$, the number of edges in $\{(x, y) \in E \mid x \in X, y \in Y\}$, is minimized.

Minimum-Width Bisection: Given $G=(V, E)$, partition $\mathrm{V}$ into disjoint subsets $\mathrm{X}$ and $\mathrm{Y}$, with $|X|=|Y|$, such that e $(\mathrm{X}, \mathrm{Y})$ is minimalized. Since this leads to equal number of modules in each partition, it is needed. The more general partitioning problem is when $\mathrm{k}$ disjoint subsets are formed.

Given two $n * n$ matrix $X=\left(x_{i j}\right)$ and $Y=\left(y_{i j}\right)$, where usually $x_{i j}, y_{i j}>0$, and the objective is:

$$
\min \left(\pi \varepsilon \mathrm{S}_{\mathrm{n}}\right) \sum_{\mathrm{i}=1}^{\mathrm{n}} \sum_{\mathrm{j}=1}^{\mathrm{n}} \mathrm{x}_{\mathrm{ij}} \mathrm{y}_{\pi(\mathrm{i}) \pi(\mathrm{j})}
$$

where $\mathrm{Sn}$ is set of all probable permutation of $(1,2 \ldots \ldots \mathrm{n})$. Sometimes there is an accessory $n * n$ matrix $Z=\left(z_{i j}\right)$, then the equation becomes,

$$
\min \left(\pi \varepsilon \mathrm{S}_{\mathrm{n}}\right) \sum_{\mathrm{i}=1}^{\mathrm{n}} \sum_{\mathrm{j}=1}^{\mathrm{n}} \mathrm{x}_{\mathrm{ij}} \mathrm{y}_{\pi(\mathrm{i}) \pi(\mathrm{j})}+\sum_{\mathrm{i}=1}^{\mathrm{n}} \mathrm{z}_{\mathrm{i} \pi(\mathrm{j})}
$$

$\boldsymbol{x}_{i j}$ represents the flow from the module i to the module $\mathrm{j}$,

$\boldsymbol{y}_{i j}$ represents the distance from the location i to the location $\mathrm{j}$,

$\boldsymbol{z}_{i j}$ represents the cost of the placing module $\mathrm{i}$ to the location $\mathrm{j}$. 


\section{MEMETIC ALGORITHM}

In evolutionary computation, the Memetic algorithms (MA) play a vital role in growing areas of research. The word MA is now broadly used as a synergy of any global search procedure with local enhancement procedures for problem search.

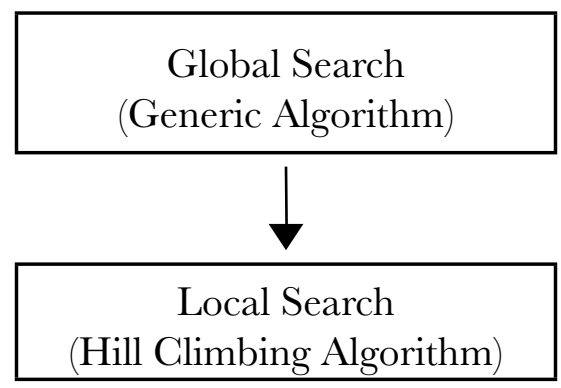

Figure 2. Memetic Algorithm.

The memetic algorithm, which is shown in Figure 2 is utilized to reduce the interconnections, i.e. min-cut problem of circuit partitioning based on a balanced limitation.

\subsection{GENETIC ALGORITHM}

A global exploration procedure to solve optimization problem, which evolves toward better solution, known as Genetic algorithm, is shown in Figure 3.

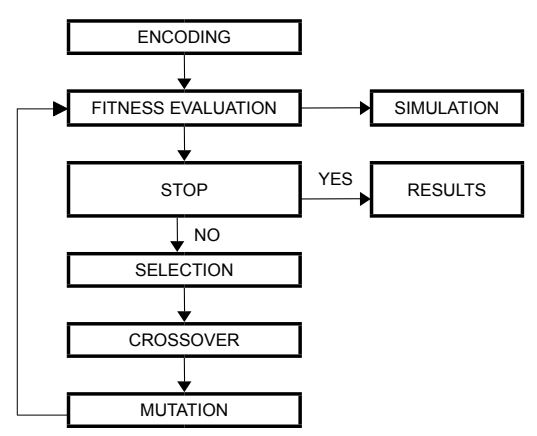

Figure 3. Genetic Algorithm- Flow Chart.

Encoding: The parameter like wire-length are represented as fixed length binary strings.

Initialization: Refers to generation of population of 'n' chromosomes randomly. Here, the population is the tentative solution for the problem. The population is here initialized by Roulette wheel Selection or Tournament methods. 
Fitness Evaluation: Evaluate the fitness $\mathrm{f}(\mathrm{x})$ of each chromosome $\mathrm{x}$ in the populace

Selection: In this process, the better fit two strings are selected as two parent chromosomes to create the offspring.

Grossover: Two parent qualities are mixed to get new children so that solution characteristics get changed. A probability is associated with this.

Mutation: With a mutation probability mutate new offspring to introduce new properties.

Termination: The process continues and replaces the existing solution until the termination condition is satisfied.

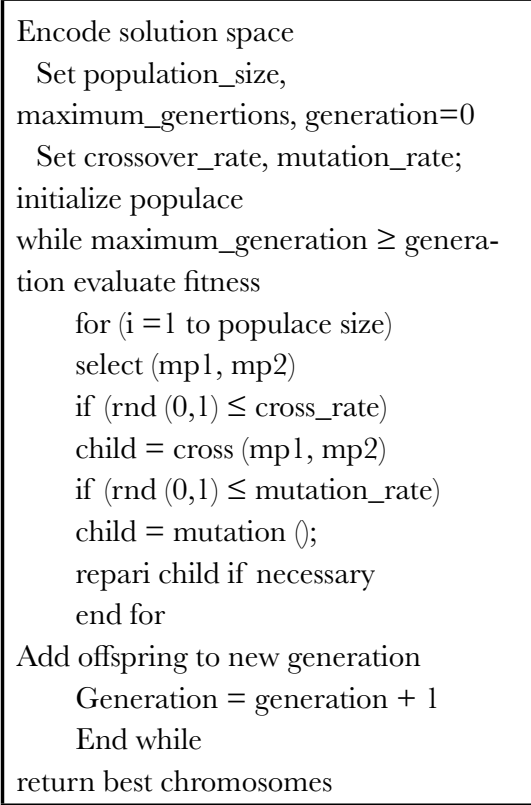

Figure 4. Pseudo code for Genetic Algorithm.

The algorithm takes specific paces, Initialization, Evaluation, Selection, Crossover, and Mutation. Every time, each person's fitness in the populace is evaluated. The fitness is typically the assessment of the target work in the issue being tackled. The best individual is preferred arbitrarily from the present populace and every individual's chromosomes and qualities are altered to make the fittest. The new populace is then used in the algorithm. The algorithm will end after predefined number of populaces are produced or achieved the optimal fitness function. 


\subsection{HILL CLIMBING ALGORITHM}

As stated in Lin and Zhu (2014), the GA is not fit for finding solutions which have closed to optimal solutions. Hence, usually GA is combined with local search algorithms like Hill climbing algorithm called Memetic Algorithms are used. In this paper we proposed a Memetic algorithm based on Genetic Algorithm and Hill climbing algorithm for circuit partitioning. Hill climbing algorithm is one of the algorithms to find the best state in optimization problems with less conditions than other techniques.

The genetic algorithm is not appropriate for fine-tuning the solution which are close to optimal. So, for fine tuning separate algorithm (local hill climbing algorithm) is used with genetic algorithm called Memetic. They have demonstrated that they are requests of greatness speedier than customary hereditary Algorithms for some issue areas. In a memetic algorithm, the population is initialized randomly or using a heuristic. Then, every individual makes nearby search to enhance its wellness. To frame another populace for the following group, higher quality solutions are chosen. The selection stage is similar stage. With two individuals selected, their chromosomes are joined to produce new individuals.

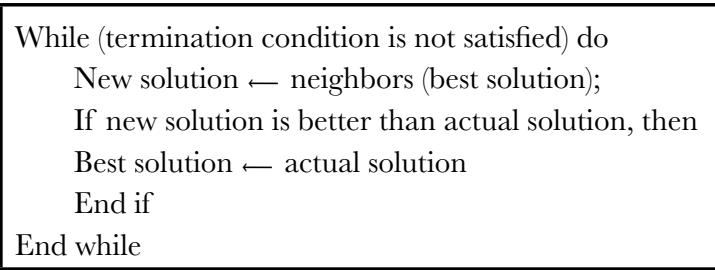

Figure 5. Pseudo Code for Hill climbing search algorithm.

The later are boosted utilizing a neighbourhood seek method. The role is to trace the local best more proficiently than the genetic algorithm. The hill climbing search algorithm proposed as a local search procedure shown in Figure 5. It is just a loop that ceaselessly goes toward expanding quality.

\section{RESULTS}

The parameter settings of iteration are varied, and the cut size is calculated. The best cost for various iterations up to 20 iterations as example, is taken in partitioning ami33 is shown in figures below: 


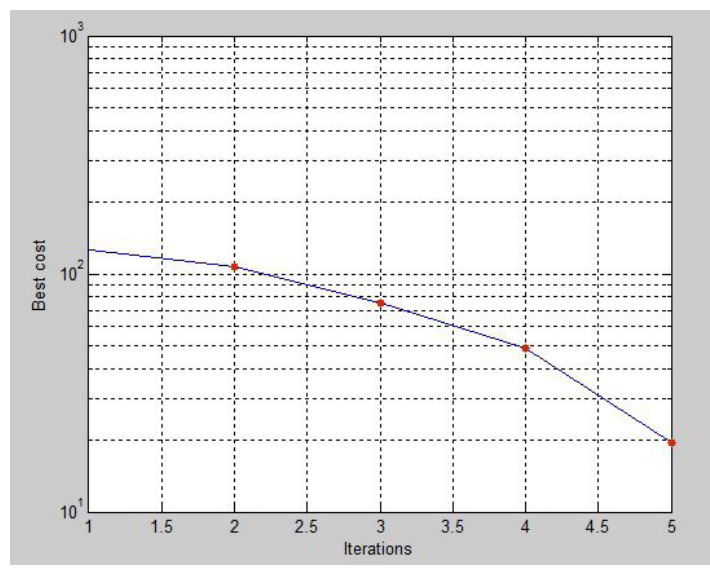

Figure 6. Iterations Vs. Best cost (for Maxlt=5).

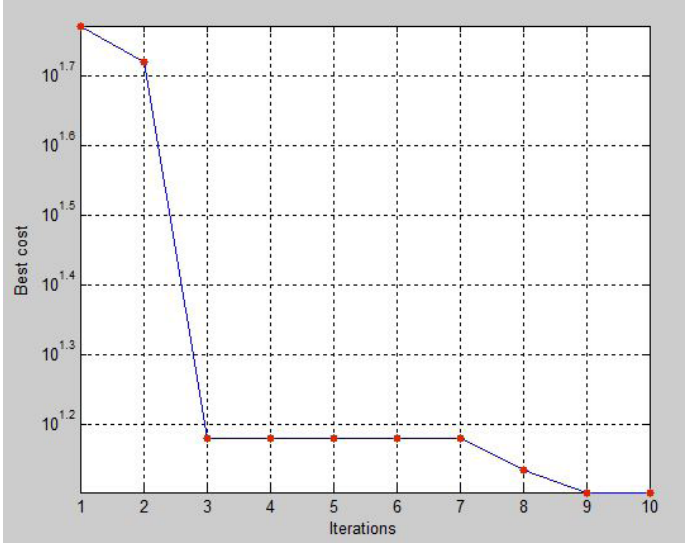

Figure 7. Iterations Vs. Best cost (for Maxlt=10).

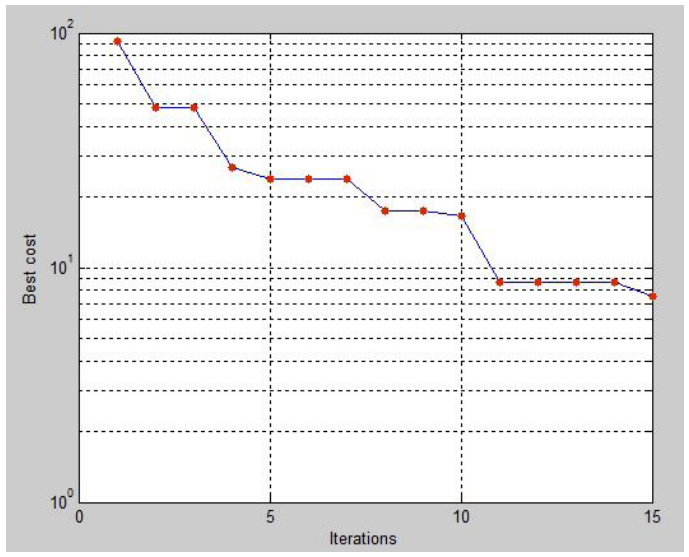

Figure 8. Iterations Vs. Best cost (for Maxlt=15). 


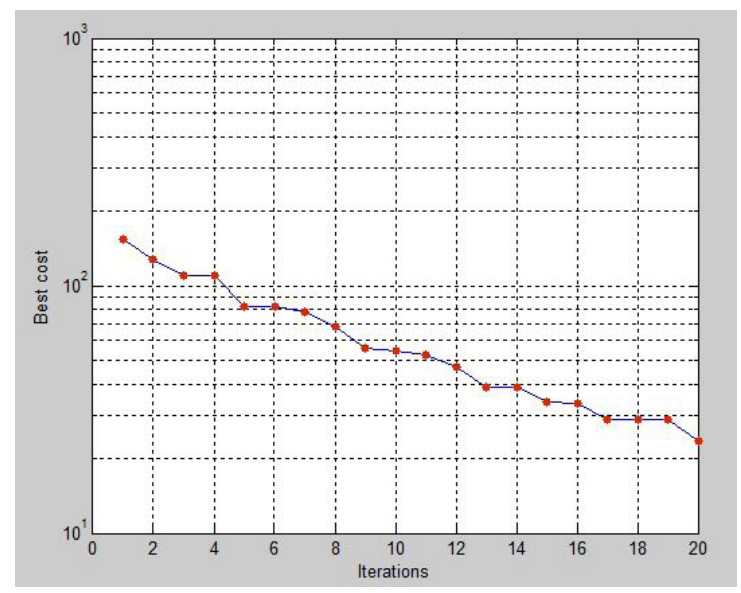

Figure 9. Iterations Vs. Best cost (for Maxlt=20).

The tabulation for memetic algorithm of min cut, max cut and average cut is shown in the Table 1.

Table 1. Min cut, max cut and average cut of partitioning.

\begin{tabular}{|c|c|c|c|c|}
\hline Iterations & Min cut & Max cut & Average cut & Memetic Mean (*106) \\
\hline 5 & 32.06 & 576.02 & 29.03 & 5.26 \\
\hline 10 & 32.38 & 544.03 & 27.2 & 5.26 \\
\hline 15 & 33.5 & 544.08 & 28.29 & 5.44 \\
\hline 20 & 32.40 & 320.00 & 19.20 & 5.44 \\
\hline
\end{tabular}

The results clearly show that the proposed work results in one of the best ways to partition a 3D IC.

\section{CONCLUSION}

The combination of genetic algorithm with local hill climbing algorithm forms a memetic algorithm which is proposed to circuit partitioning yields a major development in result quality. The experimental result shows that the algorithm provides good and consistent result. This result shows the flexibility of the memetic methodology in solving the problem of VLSI circuit netlist partitioning. 


\section{ACKNOWLEDGEMENT}

Authors want to express their gratitude to ECE Department at Kalasalingam Academy of Research and Education for allowing them to utilize the computing facilities in DST-FIST sponsored VLSI Research Laboratory. 


\section{REFERENCES}

Ababei, G., Selvakkumaran, N., Bazargan, K., \& Karypis, G. (2002). Multi-objective circuit partitioning for cutsize and path-based delay minimization. Proceedings of the 2002 IEEE/ACM International Conference on Computer-Aided Design - ICCAD 02, 181-185. https://doi.org/10.1145/774572.774599

Alpert, C.J. (1998). The ISPD98 circuit benchmark suite. Proceedings of the 1998 International Symposium on Physical Design - ISPD 98. https://doi.org/10.1145/274535.274546

Areibi, S., \& Yang, Z. (2004). Effective Memetic Algorithms for VLSI Design = Genetic Algorithms Local Search Multi-Level Clustering. Evolutionary Computation, 12(3), 327353. https://doi.org/10.1162/1063656041774947

Du, H. Q., \& Qi, J. B. (2010). Application of a Hybrid Algorithm Based on Genetic Algorithm and Hill-Climbing Algorithm to Tool Path Optimization in CNG Machining. Advanced Materials Research, 102-104, 681-685. https://doi.org/10.4028/ www.scientific.net/amr.102-104.681

Gill, S. S., Chandel, R., \& Chandel, A. (2010). Genetic Algorithm Based Approach to Circuit Partitioning. International Fournal of Computer and Electrical Engineering, 196-202. https://doi.org/10.7763/ijcee.2010.v2.136

Lin, G., \& Zhu, W. (2014). An Efficient Memetic Algorithm for theMax-Bisection Problem. IEEE Transactions on Computers, 63(6), 1365-1376. https://doi.org/10.1109/tc.2013.7

Marichelvam, M. K., Prabaharan, T., \& Yang, X. S. (2014). A Discrete Firefly Algorithm for the Multi-Objective Hybrid Flowshop Scheduling Problems. IEEE Transactions on Evolutionary Computation, 18(2), 301-305. https://doi.org/10.1109/ tevc.2013.2240304

Nagarajan, K. (2018). A Predictive Hill Climbing Algorithm for Real Valued multiVariable Optimization Problem like PID Tuning. International fournal of Machine Learning and Computing, 8(1), 14-19. https://doi.org/10.18178/ijmlc.2018.8.1.656 
Roy, S., \& Sarma, S. S. (2012). Improvement of the quality of VLSI circuit partitioning problem using Genetic algorithm. Fournal of Global Research in Computer Science, 3(12), $18-22$.

Sharma, P. K., \& Kaur, M. (2014). A Discrete FireFly Algorithm for VLSI Circuit Partitioning. 2014 International Conference on Electronics and Communication Systems (ICECS). https://doi.org/10.1109/ecs.2014.6892764

Subbaraj, P., Sivasundari, K., \& Kumar, P. (2007). An effective memetic algorithm for VLSI partitioning problem. IET-UK International Conference on Information and Communication Technology in Electrical Sciences (ICTES 2007), 667-670. https://doi. org/10.1049/ic:20070696 


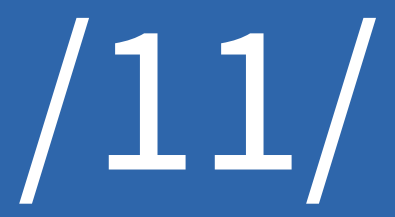




\section{MODIFIED SOBEL MASK TO LOCATE KNEE JOINT BOUNDARIES}

S. Sheik Abdullah

Research Scholar, Department of Electronics and Communication Engineering,

Kalasalingam Academy of Research and Education, Virudhunagar, (India). E-mail: sabdullah787@gmail.com ORCID: https://orcid.org/0000-0001-6765-8374

M. Pallikonda Rajasekaran

Professor, Department of Electronics and Communication Engineering, Kalasalingam Academy of Research and Education. Virudhunagar, (India). E-mail: mpraja80@gmail.com ORCID: https://orcid.org/0000-0001-6942-4512

\section{Citación sugerida:}

Abdullah, S. S., y Rajasekaran, M. P. (2020). Modified sobel mask to locate knee joint boundaries. 3C Tecnología. Glosas de innovación aplicadas a la pyme. Edición Especial, Marzo 2020, 195-205. http://doi. org/10.17993/3ctecno.2020.specialissue4.195-205

\section{Suggested citation:}

Abdullah, S. S., \& Rajasekaran, M. P. (2020). Modified sobel mask to locate knee joint boundaries. 3C Tecnología. Glosas de innovación aplicadas a la pyme. Edición Especial, Marzo 2020, 195-205. http://doi. org/10.17993/3ctecno.2020.specialissue4.195-205 


\section{ABSTRACT}

Sobel masking algorithm is a very important technique to detect edges in an image. Comparing the Sobel gradient operator with other edge/boundary detection operators used repeatedly; Making an additional study on the traditional Sobel gradient operator, the benefits of Sobel mask are its quick speed of detecting edges. Meanwhile, it has also an impact on suppressing and smoothing noise. In addition, Sobel operator has a standard effect on detecting the edges. Although Sobel gradient operator has some advantages in different aspects, it exists some problems. The Existing Sobel masking technique is a type of edge detection in vertical and horizontal directions only and it ignores the boundary points in other directions. It cannot attain a true location of edge points in an image. In this paper, the existing sobel technique is improved by adding an increase of 315 degrees and 360 degrees in horizontal and vertical directions. This will have an effect of detecting the knee joint space of osteoarthritis. According to simulation results, they show this method is very simple and feasible, and the outcomes are more abundant than traditional Sobel edge detection. In this paper edge detection and noise interference problems are improved.

\section{KEYWORDS}

Osteoarthritis, Sobel mask, Image Processing Techniques. 


\section{INTRODUCTION}

In digital image processing, edge/boundary feature is one of the very important characteristics of the image, and it is a significant part of image processing, analyzing, pattern recognition and computer vision. Edge detection outcomes affect further image analyzing and pattern/texture recognition directly (Amer \& Abushaala, 2015). In recent days, Image edge detection has become the main research theme in image processing technology. With the advance of science and technology, researchers have analyzed and proposed some techniques for the detection of edges in an image and assessment of edge detection. At the same time, these edge/boundary recognition methods are applied to the area of digital vision and pattern recognition, which make the use of edge detection technology more broadly. Over the years, segmentation of an image has been creating more and more attention. Lots of image segmentation techniques have been put forward. They can be divided into different methods like bit threshold method, edge detection method and regional growth method (Argyle, 1971; Canny, 1989). Edge detection method comprises of: edge detection operator which contains mask like Roberts operator, Prewitt operator, LOG operator and Sobel operator (Abbasi \& Abbasi, 2007). Sobel mask is slightly better than others. The classical Sobel technique also has some problems such as it is sensitive to the vertical and horizontal direction only (Lakshmi \& Sankaranarayanan, 2010). However, the information in the image is not restricted to the horizontal and vertical directions; it can make an element of the image information lose. In this paper, a new improved operator is proposed to detect more image information. In the modified Sobel operator, 2 direction patterns (315 degrees and 360 degrees) are added to get multi-directional image acquisition. Then calculate the threshold by using the Otsu method and refine the detected rough edges by using the method to achieve the results of image edge detection. Edge detection effect can be achieved better by using the Matlab simulation method.

\section{LITERATURE SURVEY-COMPARISION OF TRADITIONAL EDGE DETECTION OPERATORS}

Roberts operator: It did not pass smooth analysis, so it is very sensitive to the noise.

Prewitt operator and Sobel operator: extraction of edge/boundaries effect is almost the same (Lakshmi \& Sankaranarayanan, 2010; Abbasi \& Abbasi, 2007). Sobel operator is a weighted average filter, Prewitt operator is an average filter; Sobel operator have better 
detection effect on images which have low level noise, but the detection of the edge effect is not clear.

LOG operator: detecting edges by using second order derivatives zero crossing edge method (Yu-quian, Wei-hua, Zhen-cheng, Jing-tian, \& Ling-yun, 2005). Smoothing effect is more important, noise removal is improved, but the loss of information in an image is higher, the edge accuracy is lower. So there is a challenge between placing edge accuracy and removing noise level.

\section{TRADITIONAL SOBEL OPERATOR}

Sobel operator, because of its task in the pattern is small, the computation is also very small, and the image information of the shape can be attained. Operator template size is even, the pending pixel cannot be placed in the center position of the template. Sobel differential mask is a differential mask of $3 \times 3$ size template (Argyle, 1971). The expressions of formula as follow:

$$
\begin{array}{r}
G x(u, v)=f[u-1, v+1]+2 * f[u, v+1]+f[u+1, v+1]- \\
f[u-1, v-1]-2 * f[u, v-1]-f[u+1, v-1] \\
f[u-1, v-1]-2 * f[u, v-1]-f[u+1, v-1] \\
G x(u, v)=f[u-1, v-1]+2 * f[u+1, v]+f[u+1, v+1]- \\
f[u-1, v-1]-2 * f[u-1, v]-f[u-1, v+1] \\
f[u-1, v-1]-2 * f[u-1, v]-f[u-1, v+1]
\end{array}
$$

The convolution template of the Sobel operator is expressed as the formula

$$
G x=\left[\begin{array}{rrr}
-1 & -2 & -1 \\
0 & 0 & 0 \\
1 & 2 & 1
\end{array}\right] \quad G y=\left[\begin{array}{rrr}
-1 & 0 & 1 \\
-2 & 0 & 2 \\
-1 & 0 & 1
\end{array}\right]
$$

The calculating steps of Sobel operator: first, the edge detection image is divided into matrix form 


$$
\left[\begin{array}{ccc}
f(x, y) & f(x+1, y) & f(x+2, y) \\
f(x, y+1) & f(x+1, y+1) & f(x+2, y+1) \\
f(x, y+2) & f(x+1, y+2) & f(x+2, y+2)
\end{array}\right]
$$

Multiply the vertical direction by horizontal direction of the template,

$$
\begin{aligned}
& F x=G x . * A \\
& F x=G y \cdot * A
\end{aligned}
$$

gradient size calculation, as shown in the formula

$$
G=\sqrt{G x^{2}+G y^{2}}
$$

The formula for calculating the gradient direction is shown in the formula

$$
\theta=\tan ^{-1}(G y / G x)
$$

The Sobel mask set up the weighted local average, The operator not only influences the edge detection of an image but also hold back the noise further, but the edge is wider. The basic idea of Sobel operator algorithm: The edge of the image is situated at the place in which the brightness varies significantly (Kalpana \& Padmaa, 2014), the gray value of pixels exceeds a set threshold depending on the specific steps for the edge (Xing, 2005). The specific steps of the Sobel operator algorithm are as follows:

- Moving the horizontal and vertical direction templates from right to left, from top to bottom, and moving from one pixel to another.

- Multiplying the pixel values in the image with operator coefficient.

- Calculated gradient value is the new gray value by using 2 convolution values.

\section{IMPROVED SOBEL OPERATOR}

Adding 315 degrees and 360 degrees with respect to the template in a basis of the traditional Sobel operator, the direction template is changed into two directions ( $G x=315$ degree, $G y=360$ degree): the horizontal and vertical direction with respect to 315 degrees and 360 degrees. It 
improved the weights of the new template in the direction of boundaries. Specific details are as follows:

According to the calculation of the two template directions and calculating an image point by point, the maximum value is observed as the pixel gray values. According to the threshold setting, the edge point is determined.

$$
\begin{gathered}
S x(o, p)=f[o+2, p+1]+2 * f[o+2, p+2]+f[o+1, p+2]- \\
f[o+1, p]-2 * f[o, p]-f[o, p+1] \\
f[o+1, p]-2 * f[o, p]-f[o, p+1] \\
S x(o, p)=f[o, p+2]+2 * f[o+1, p+2]+f[o+2, p+2]- \\
f[o, p]-2 * f[o+1, p+2]-f[o+2, p] \\
f[o, p]-2 * f[o+1, p+2]-f[o+2, p]
\end{gathered}
$$

Start

$S x=\left[\begin{array}{rrr}-3 & -1 & 0 \\ -1 & 0 & 0 \\ 0 & 1 & 2\end{array}\right] \quad S y=\left[\begin{array}{ccc}-1 & -2 & -1 \\ 0 & 0 & 0 \\ 1 & 2 & 1\end{array}\right]$

\section{Convert RGB image to grayscale}

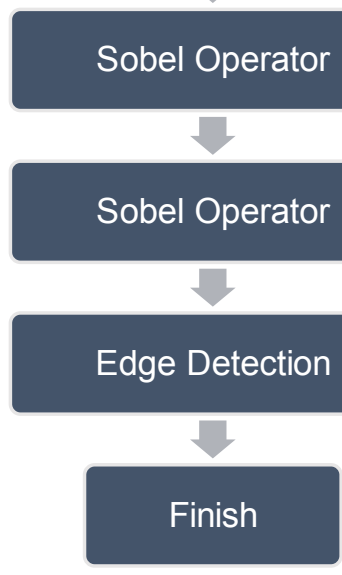

Figure 1. Flowchart of proposed system. 


\section{RESULTS}

Edge detection process followed below:

Step 1: Set Threshold value $\mathrm{T}=255$.

Step 2: If Gradient value $(\mathbf{S})<255$ is less than the Thresh, considered as 1 , other than are 0 (value below 255 set to be 0 ).

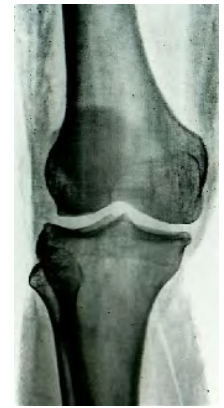

Figure 2. Original Image.

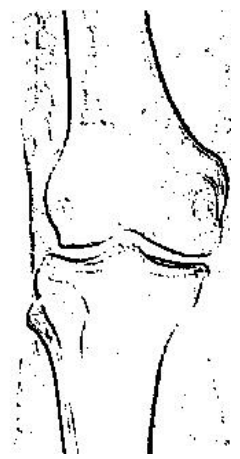

Figure 4. Sobel Operator.

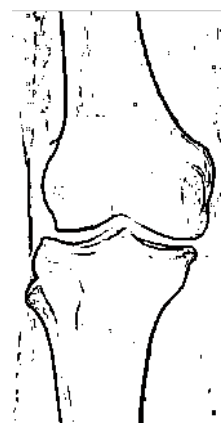

Figure 6. Prewitt Operator.

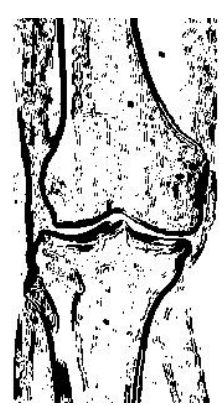

Figure 3. Proposed System.

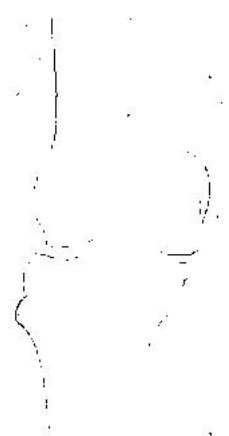

Figure 5. Roberts Operator.

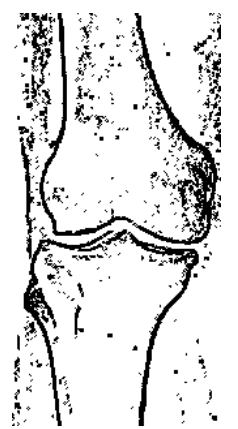

Figure 7. Homogeneity Operator. 
Table 1. PSNR comparison of different operators.

\begin{tabular}{|c|c|c|c|c|c|c|}
\hline $\begin{array}{c}\text { Test } \\
\text { images }\end{array}$ & Sobel & Zero Cross & Prewitt & Roberts & Homogeneity & $\begin{array}{c}\text { Modified } \\
\text { Sobel }\end{array}$ \\
\hline 01 & +19.39 & +13.48 & +18.51 & +14.25 & +10.23 & +20.95 \\
\hline 02 & +19.58 & +13.92 & +18.76 & +14.73 & +10.18 & +21.34 \\
\hline 03 & +19.63 & +13.79 & +13.71 & +14.63 & +10.27 & +21.15 \\
\hline 04 & +19.68 & +13.86 & +18.80 & +15.21 & +10.20 & +21.22 \\
\hline 05 & +20.03 & +13.76 & +18.96 & +14.32 & +10.31 & +21.49 \\
\hline 06 & +19.99 & +13.84 & +19.22 & +16.23 & +10.22 & +21.30 \\
\hline 08 & +19.88 & +13.86 & +19.00 & +16.10 & +10.19 & +21.45 \\
\hline 10 & +19.43 & +13.87 & +18.63 & +15.34 & +10.21 & +21.03 \\
\hline 11 & +19.63 & +13.67 & +18.72 & +15.64 & +10.41 & +21.21 \\
\hline 09 & +19.13 & +13.82 & +18.16 & +13.62 & +10.23 & +20.86 \\
\hline
\end{tabular}

\section{CONCLUSION}

This paper analyzes the classic sobel edge detection algorithm and improves the algorithm from the gradient calculation. The improved algorithm is realized that result outcomes prove that the modified algorithm is better and clearer on the edge detection of the image. From experiment, it proved that this proposed system is better than the traditional Sobel operator in image edge detection and achieves the specific accurate detection and reduces the loss of edge. The experiments show that the method provided in this paper is feasible. Improve the masking performance by increasing PSNR value and detect finite boundaries/ edges of intra articular space in future. 


\section{REFERENCES}

Abbasi, T. A., \& Abbasi, M. U. (2007). A novel FPGA-based architecture for Sobel edge detection operator. International Fournal of Electronics, 13(9), 889-896. https://doi. org/10.1080/00207210701685253

Amer, G. M. H., \& Abushaala, A. M. (2015). Edge Detection Methods. In G. Deng, Z. Liu. Comparison and Analysis for Edge Detection Algorithms based on SQI Image Enhancement. IEEE, 3615-3617.

Argyle, E. (1971). Techniques for edge detection. IEEE proceedings, 59, 285-286.

Ganny, J. (1989). A computational approach to edge detection. IEEE Transactions on Pattern Analysis and Machine Intelligence, PAMI-8(6), 679-698. https://doi.org/10.1109/ TPAMI.1986.4767851

Kalpana, Y. B., \& Padmaa, M. V. (2014). An efficient edge detection algorithm for flame and fire image processing. In 2014 International Conference on Communication and Signal Processing, 696-700. https://doi.org/10.1109/ICGSP.2014.6949932

Lakshmi, S., \& Sankaranarayanan, V. (2010). A study of Edge Detection Techniques for Segmentation Computing Approaches. IfCA Special Issue on Computer Aided Soft Computing Techniques for Imaging and Biomedical Applications. https:/ / pdfs.semanticscholar. org/3c0e/8119096edd337002d8430c6c6ad69f126520.pdf

Rama Bai, M. (2010). A new approach for border extraction using morphological methods. International Fournal of Engineering Science and Technology, 2, 3832-3837.

Xing,J. (2005). Digital image edge detection based on Sobel operator. Fournal of microcomputer development, 18-19.

Yu-quian, Z., Wei-hua, G., Zhen-cheng, G., Jing-tian, T., \& Ling-yun, L. (2005). Medical Images Edge detection Based on mathematical Morphology. In 2005 IEEE Engineering in Medicine and Biology 27th Annual Conference, Shanghai, China. https://doi. org/10.1109/IEMBS.2005.1615986 
Zhang, R., Zhao, G., \& Su, L. (2005). A New Edge Detection Method in Image Processing. IEEE International Symposium on Communications and Information Technology. ISCIT, 1, 445448. https://doi.org/10.1 109/ISCIT.2005.1566889 


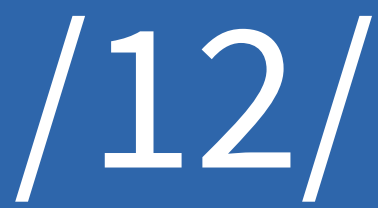




\title{
HUMAN 2D EAR BIOMETRIC RECOGNITION BASED ON CONTOUR MATCHING TECHNIQUE
}

\author{
Alagarsamy Santham Bharathy \\ Department of Electronics and Communication Engineering, \\ School of Electronics and Electrical Technology, \\ Kalasalingam Academy of Research and Education, \\ Krishnankoil, Virudhunagar Dt., (India). \\ E-mail: santhembharathy@gmail.com ORCID: https://orcid.org/0000-0003-0978-3905 \\ Kalpana Murugam \\ Department of Electronics and Communication Engineering, \\ School of Electronics and Electrical Technology, \\ Kalasalingam Academy of Research and Education, \\ Krishnankoil, Virudhunagar Dt., (India). \\ E-mail: drmkalpanaece@gmail.com ORCID: https://orcid.org/0000-0002-5121-0468
}

Recepción: 05/12/2019 Aceptación: 03/01/2020 Publicación: 23/03/2020

\section{Gitación sugerida:}

Bharathy, A. S., y Murugam, K. (2020). Human 2D Ear Biometric Recognition Based on Contour Matching Technique. 3C Tecnología. Glosas de innovación aplicadas a la pyme. Edición Especial, Marzo 2020, 207-217. http://doi.org/10.17993/3ctecno.2020.specialissue4.207-217

\section{Suggested citation:}

Bharathy, A. S., \& Murugam, K. (2020). Human 2D Ear Biometric Recognition Based on Contour Matching Technique. 3C Tecnología. Glosas de innovación aplicadas a la pyme. Edición Especial, Marzo 2020 , 207-217. http://doi.org/10.17993/3ctecno.2020.specialissue4.207-217 


\section{ABSTRACT}

This paper presents, the Ear detection biometric is obtainable utilizing normal ear method to detection, which is motivated through normal face acknowledgment methods. This work proposed another ear correlation method dependent on template expansion. The work is connected with ear database given by USTB China on which, the work delivered 100\% exactness more than 180 ear images.

\section{KEYWORDS}

Image Processing, Ear Images, Feature Extraction, Contour Matching. 


\section{INTRODUCTION}

In the recent science technology biometrics where an element is recognized based on physical highlights or conduct qualities (Basit, Javed, \& Anjum, 2005). Physical attributes incorporate unique face, retina, finger, palm print, iris, and ear with so forth while behavioral qualities comprise of step acknowledgment, voice, odour acknowledgment, with mark confirmation. The acquired biometric outcomes are utilizing solo or different methods. The accomplished outcomes show that biometric methods be considerably extra exact with precise over conventional systems. But accuracy, it has been dependably sure issues which stay related to current customary methods. For instance, think about belonging and information. Both can be shared, stolen, overlooked, copied, lost or removed. Anyway, the peril is limited in the event of biometric implies (Moreno, Sanchez, \& Velez, 1999). The biometrics work is amiable within a wide range of safety frameworks. By means of the dangers/progresses of innovations, and it's needed a constant to look at new methods for utilizing like remain solitary relevancies or related to current frameworks. To incorporate any new category of biometric, the state necessary is that it ought to be general, unmistakable, eternal and collectible for example every people should have those highlights (widespread) and highlights ought to recognizable in support of every person (particular). The highlights ought didn't to shift (everlasting) and it must be anything but difficult to get required data from these highlights (collectible) (Jain, Hong, \& Pankati, 2000). Clearly, ears are an unmistakable element of all people making it all around satisfactory. Ear biometrics has a few points of interest over whole face: decreased position able goals, a progressively equal appropriation hue and reduce fluctuation with demeanors and direction of face. In this proposed work, another ear acknowledgment strategy is planned dependent by and large ear; it is connected for individual ID. The remaining of this paper is sorted out as pursues. In section 2 foundation and related work regarding ear acknowledgment are given. Section 3 incorporates pre-handling pursued by highlight origin and coordinating in section 4 . The section 5 test outcomes with talk are accounted for an indefinite section 6 ends be made.

\section{RELATED WORK}

The first ear was utilized for acknowledgments for individual was elaborated in Iannarelli (1989) who utilized labor-intensive methods toward distinguish ear pictures. Tests of 
more than ten thousands ears were concentrated to demonstrate the uniqueness of ears. Arrangement of ear could not modify profoundly after some time. The restorative writing (Victor, Bowyer, \& Sarkar, 2002) gives data that ear development is corresponding later than initial 4 months in birth and modifies are not detectable from the age eight to seventy. In paper, Chang, Bowyer, Sarkar, and Victor (2003), and Chen and Bhanu (2005) utilized Eigen ear image for distinguishing proof. The outcomes got be diverse in the two types. In Kumar (2012), Miyazawa, Ito, Aoki, Kobayashi, and Nakajima (2008), Ito, Iitsuka, and Aoki (2009), Ansari and Gupta (2007), and Hurley, Nixon, and Carter (2005) outcomes demonstrate no distinction in face and ear execution as Victor's outcomes demonstrate that ear execution is more awful over face. As per in Yan and Bowyer (2007), Joshi and Chauhan (2011), Gonzalez, Woods, and Eddins (2004), and Tang (2016), the distinction in result may be because of utilization of various picture quality. As in Kumar (2012), utilized 2D force pictures of ears by means of 3 neural methodologies (Weighted Bayesian, Bayesian, Borda) for acknowledgment. In this work, three pictures of every individual as of 60 individuals were utilized to assess the acknowledgment.

\section{PROPOSED SYSTEM}

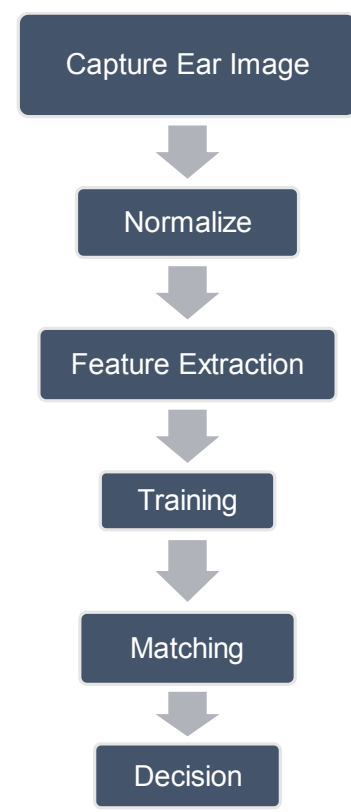

Figure 1. Process. 
Each picture is experienced the accompanying strides before highlight extraction. Ear picture is edited physically from the caught head picture of an individual. Edited ear picture is resized. The hued picture is changed over into grayscale. Concentrate the highlights structure grayscale picture utilizing vigilant edge finder and spare as the paired picture. Manual trimming has been done in the work in light of the fact that robotized ear editing is under the procedure. The sizes of the edited ear picture are extraordinary. So as to locate a similar amount of highlights as of every ear picture, rearranging the pictures to a remarkable fixed size of $80 * 150$ pixels is completed. Every picture is changed over as of color to grayscale. At that point, it is sent in support of the component origin part by the Canny edge finder. In Figure 2 exhibits yield toward finish the pre-handling step. In Figure 2(a) demonstrates the genuine picture in the catalog with the trimmed picture is obvious in Figure 2 (b). Figure 2 (c) and Figure 2 (d) are the resized edited picture with color and grayscale individually. Figure 2 (e) is the genuine element removed after pre-preparing.

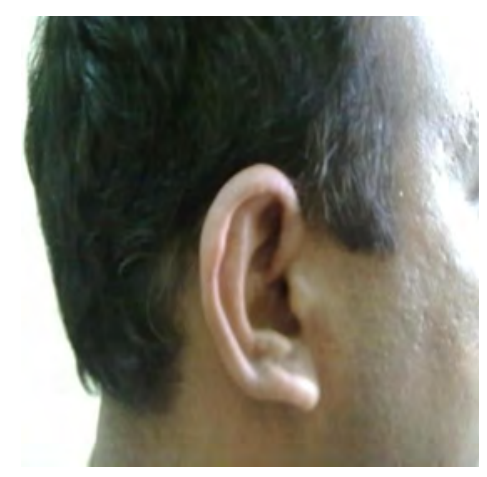

Figure 2 (a). Real Image.

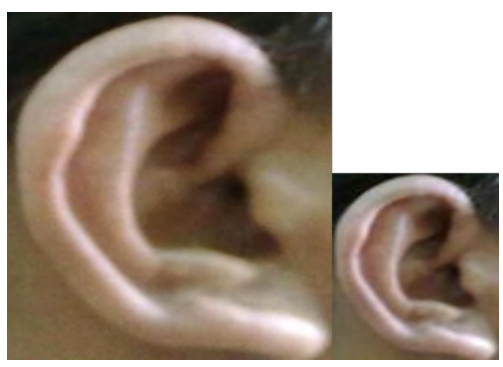

Figure 2 (b). Image Cropped. Figure 2 (c). Image Resized. 


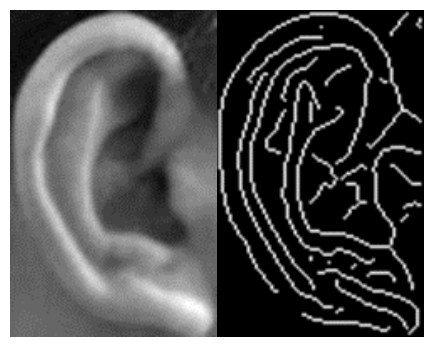

Figure 2 (d). Image Gray Scale. Figure 2 (e). Image Binary with real Features.

\subsection{FEATURE LEVEL EXTRACTION WITH MATCHING}

While the portioned ear can be legitimately utilized during the coordinating stage, most frameworks separate a striking arrangement of highlights to speak to the ear. Highlight extraction alludes to the procedure where the sectioned ear is diminished to a numerical model (e.g., an element vector) that abridges the prejudicial data. In the wake of normalizing the ear pictures, the following stage is including extraction and coordinating. Existing technique vigilant edge finder has been utilized for highlight extraction. Another strategy is proposed for ear correlation dependent by and large ear picture. In this methodology, every ear picture highlights are as a parallel lattice of $80 \times 150$. To build the thickness of ear picture highlights, enlargement activity has been achieved on each ear picture. In Figure $3(\mathrm{a})$ is the real ear picture highlights and $3(\mathrm{~b})$ is the enlarged picture. ' $\mathrm{N}$ ' is a number of the expanded twofold picture of a similar individual with various variety has been utilized to figure normal ear picture. Determined normal ear picture has been spared as a twofold framework for the layout. These exploration works, 180 ear pictures of 60 people (three pictures of every individual) has been utilized. The 3 pictures of every individual have been utilized for normal picture figuring and spare as a double framework of $80 \times 150$ which is utilized as a layout. The ensuing calculation has planned for ear perceiving.

Stage 1: calculate complete number of pixel in twofold normal ear picture format.

Stage 2: achieve bitwise intelligent OR activity among the normal double picture and inquiry picture. Tally yet again the number of resultant.

Stage 3: the all-out number of ones include in Stage 2 is same, which is included in stage 1, at that point show the note ear is perceiving through the personality of layout and outlet. 
Stage 4: if all out no of one's includes in Stage 2 is fewer, at that point and equivalent to the quantity of include in stage 1 in addition to limit esteem (for this situation edge worth is 200 pixels) at that point question ear picture is perceived and exit.

Stage 5: Check on the off chance that it is last normal ear layout, on the off chance that indeed, at that point go to Stage 6 generally go to Step 1 and contrast question picture and another ear format.

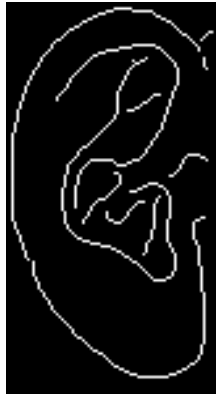

Figure 3 (a). Image in Binary by means of real Features.

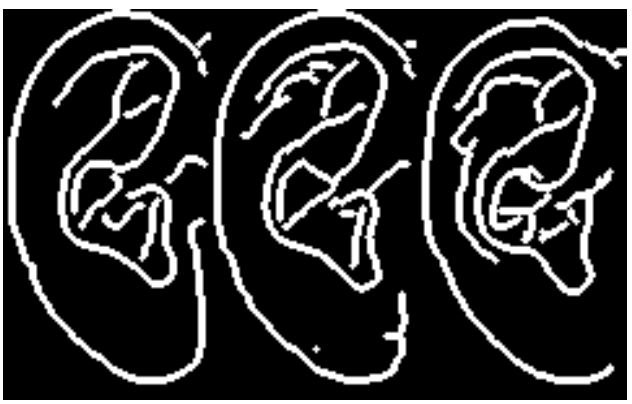

Figure 3 (c). 3 Dilated picture utilized in Average Image Calculation.

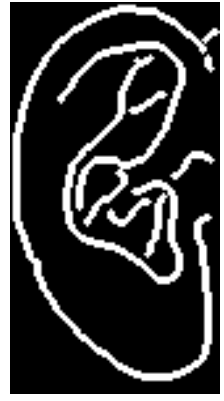

Figure 3 (b). Image Dilated Features.

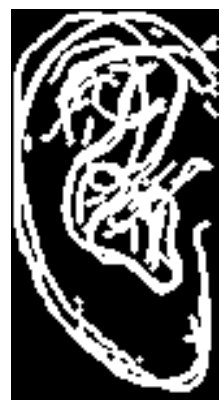

Figure 3 (d). Real Image Average.

\section{RESULTS AND DISCUSSION}

The planned strategy is actualized in MATLAB 2017 version on a laptop. In the examination, ear database from the USTB has been utilized. The databases enclose a sum of 200 pictures with $80 \times 150$ pixels goals. A lot of 60 individuals has been utilized for examinations having at least three pictures each. Three pictures of every individual have been utilized for normal picture computation. The resultant picture has been utilized as a format for ear acknowledgment. 


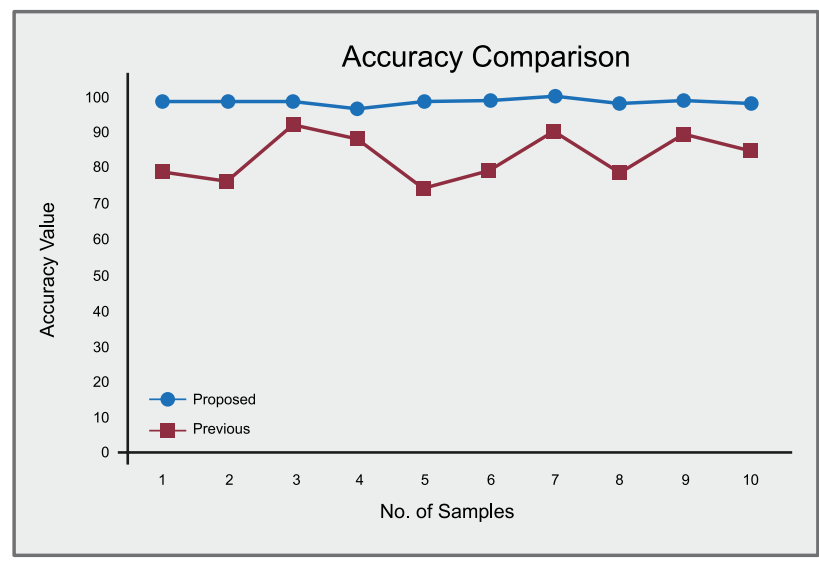

Figure 4. Accuracy comparison Ear Recognition.

Time investigation has demonstrated Figure 4, the time examination among time in second with time taking to perceive explicit ear, at $2.27 \mathrm{GHz}$ Intel 3i processor, inquiry ear picture correlation time and putting away normal ear format is 0.108 Second. In this analysis, 180 ear pictures of 60 people, three pictures of every individual have been utilized for normal picture count. The ear acknowledgment rate is $100 \%$ percent more than 180 pictures. 20 pictures of 20 people, which isn't taking an interest in normal picture computation likewise delivered $90 \%$ exactness by utilizing a limit esteems $\mathrm{TH}=173$. In this examination work, test on possess database is under-preparing, It is normal that as the number of ear picture increment for normal picture computation, the acknowledgment rate will increment.

\section{CONCLUSION}

Ear biometrics got consideration regarding the examination as of late. In this paper, another technique for human acknowledgment is proposed dependent by and large ear pictures. Ear pictures are trimmed physically and resized to a fixed size pursued by the change to grayscale. After that Canny edge identifier is utilized to remove the element from the picture. Database pictures are prepared and put away as a normal ear picture. Results got are promising and empowering with right acknowledgment rate just as the time required. Results will get better if number of ear pictures increment in normal picture count. 


\section{REFERENCES}

Ansari, S., \& Gupta, P. (2007). Localization of ear using outer helix curve of the ear. International Conference on Computing Theory and Applications (ICCTA), pp. 688-692. https://doi.org/10.1109/ICGTA.2007.82

Basit, A., Javed, M. Y., \& Anjum, M. A. (2005). Efficient iris recognition method for human identification. International Scholarly and Scientific Research \& Innovation, 1(4), 2426. https://publications.waset.org/15966/pdf

Chang, K., Bowyer, K. W., Sarkar, S., \& Victor, B. (2003). Comparison and Combination of Ear and Face Images in Appearance-Based Biometrics. IEEE Transactions on Pattern Analysis and Machine Intelligence, 25(9), 1160-1165. https://doi. org/10.1109/TPAMI.2003.1227990

Chen, H., \& Bhanu, B. (2005). Contour Matching for 3D Ear Recognition. Seventh IEEE Workshops on Applications of Computer Vision (WACV/MOTION'05), 1, 123-128. https:/ / doi.org/10.1109/acvmot.2005.38

Gonzalez, G. R., Woods, R. E., \& Eddins, S. L. (2004). Digital Image Processing Using $\operatorname{MATLAB}(R)$. Pearson Education.

Hurley, D. J., Nixon, M. S., \& Carter, J. N. (2005). Force field feature extraction for ear biometrics. Computer Vision and Image Understanding, 98(3), 491-512. https://doi. org/10.1016/j.cviu.2004.11.001

Iannarelli, A. V. (1989). Ear Identification. Paramont Publishing Company.

Ito, K., Iitsuka, S., \& Aoki, T. (2009). A palmprint recognition algorithm using phasebased correspondence matching. In 16 th IEEE International Conference on Image Processing (ICIP). https://doi.org/10.1109/ICIP.2009.5413740

Jain, A., Hong, L., \& Pankati, S. (2000). Biometric Identification. Communications of the ACM, 43(2), 91-98. https://doi.org/10.1145/328236.328110 
Joshi, K., \& Chauhan, N. C. (2011). A Template Matching and Support Vector Machine based Approach for Human Ear Detection and Varification. International Conference on Information, Signal and Communication. https://www.researchgate.net/ publication/281741782_A_Template_Matching_and_Support_Vector_Machine_ Based_Approach_for_Human_Ear_Detection_and_Verification

Kumar, A. (2012). Can We Use Minor Finger Knuckle Images to Identify Humans? IEEE Fifth International Conference on Biometrics: Theory, Applications and Systems (BTAS), pp. 5560. https://doi.org/10.1109/crprw.2014.21

Miyazawa, K., Ito, K., Aoki, T., Kobayashi, K., \& Nakajima, H. (2008). An Effective Approach for Iris Recognition Using Phase-Based Image Matching. IEEE transactions on pattern analysis and machine intelligence, 30(10), 1741-1756. https://doi.org/10.1109/ TPAMI.2007.70833

Moreno, B., Sanchez, A., \& Velez, J. F. (1999). On the Use of Outer Ear Images for Personal Identification in Security Applications. Proceedings IEEE 33rd Annual 1999 International Carnahan Conference on Security Technology (Cat. No.99CH36303). https:/ / doi. org/10.1109/CCST.1999.797956

Tang, T. (2016). Biometric Recognition Using Wavelet Domain Features. Electrical and Electronics Engineering An International Fournal (ELELFU), 5.

Victor, B., Bowyer, K., \& Sarkar, S. (2002). An Evaluation of Face and Ear Biometrics. Proceedings 16th International Conference Pattern Recognition, pp. 429-432. https://doi. org/10.1109/ICPR.2002.1044746

Yan, P., \& Bowyer, K. W. (2007). Biometric recognition using 3D ear shape. IEEE Transactions on Pattern Analysis and Machine Intelligence, 29(8), 1297-1308. https://doi. org/10.1109/TPAMI.2007.1067 


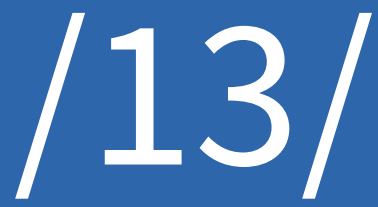




\title{
DESIGN OF MODIFIED MARCH-C ALGORITHM AND BUILT-IN SELF-TEST ARCHITECTURE FOR MEMORIES
}

\author{
G. Karthy \\ B.E., M. Tech., Assistant Professor, ECE, \\ Kalasalingam Academy of research and education. \\ Krishnankoil, (India). \\ E-mail: g.karthy@klu.ac.in ORCID: https://orcid.org/0000-0003-0084-4185 \\ P. Sivakumar \\ B.E., M.Tech., Ph.D., Professor, ECE, \\ Kalasalingam Academy of Research and Education. \\ Krishnankoil, (India). \\ E-mail: siva@klu.ac.in ORCID: https://orcid.org/0000-0003-1328-8093
}

\section{Citación sugerida:}

Karthy, G., y Sivakumar, P. (2020). Design of Modified March-C Algorithm and Built-in self-test architecture for Memories. $3 C$ Tecnología. Glosas de innovación aplicadas a la pyme. Edición Especial, Marzo 2020, 219-229. http://doi.org/10.17993/3ctecno.2020.specialissue4.219-229

\section{Suggested citation:}

Karthy, G., \& Sivakumar, P. (2020). Design of Modified March-G Algorithm and Built-in self-test architecture for Memories. 3C Tecnología. Glosas de innovación aplicadas a la pyme. Edición Especial, Marzo 2020, 219-229. http://doi.org/10.17993/3ctecno.2020.specialissue4.219-229 


\section{ABSTRACT}

Semiconductor Memories is a pivotal aspect as its technology growth increases. RAM, ROM, DRAM, etc., are the different types of memory and it becomes difficult to test the memory because of the complexity of the design increases day by day. The testing of memory is very difficult as it's required many test patterns. In this paper, a new test architecture is designed using a response analyzer and checker to detect a fault on a chip, and the modified MARCH C algorithm is also proposed to check the fault in the memory in the shortest time.

\section{KEYWORDS}

RAM, SOC, Response analyzer, Checker, March algorithm. 


\section{INTRODUCTION}

Testing the complete memory is a difficult task. Testing can be done with help of fault models in the Built-in self-test (BIST) architecture. Many fault models are available to test the memory. Here we have used traditional faults models to test the memory. Out of various available algorithms MARACH algorithms provides better fault detection and coverage. In this paper, we proposed a new architecture that consists of the checker, response analyzer, memory unit and a BIST controller with Modified March C algorithm. By using a checker, we can get more précised output.

\section{PROBLEM STATEMENT}

Memory testing is used to identify that the memory is capable of writing and reading the correct data or not. March based algorithms can identify and locating the fault types which can help to check the design and manufacturing errors. The quality of the test is strongly dependent on the fault model in terms of its fault coverage, its test length as well as the test time required.

In this paper Modified MARCH C- the algorithm is implemented to detects the maximum fault. In addition to that Response analyzer and Checker are included in this architecture to identifies more faults with high precision.

\section{TYPES OF FAULT IN THE MEMORY}

There are 3 types functional faults models involved in the memory:

1. Memory cell faults.

2. Address Decoder faults.

3. Dynamic faults.

\subsection{MEMORY CELL FAULTS}

This type of faults forces the contents from 0 to 1 or does not change the contents. Types are SAF- Stuck at fault, SOF-Stuck at open fault, TF - Transition fault, DRF-Data retention 
fault, CF- Coupling fault, BF-Bridging fault BF, NPSF-Neighborhood Pattern Sensitive Fault, Active (Dynamic) NPSF, Passive NPSF, Static NPSF.

\subsection{ADDRESS DECODER FAULTS (AFS)}

It occurs in the address, it can be:

Cell not accessed by an address, many cells are accessed by an address, cell accessed by many addresses.

\subsection{DYNAMIC FAULTS}

1. Recovery faults: Part of the memory cannot recover fast enough from a previous state.

2. Disturb faults: victim cell forced to 1 or 0 when we read or write aggressor cell (maybe the same cell).

3. Data Retention faults: Because memory loses its content spontaneously, data cannot be retrieved.

\section{MARCH ALGORITHMS}

The fixed sequence of read/write operations is carried out to check whether the memory cell is good. The targeted fault model decides the real number of write/read operations and the order of the operations. March tests are the most commonly used memory test algorithms, in which there are fixed sequences of March elements. Then March element is applied to a cell in memory one by one. The operation can be in either descending or ascending address order. The notations of the March algorithm are summarized below:

$\uparrow:$ address sequence changes in ascending order

$\downarrow$ : address sequence changes in descending order

$\uparrow:$ address sequence can change either way

R0: read operation (reading a 0 from a cell)

R1: read operation (reading a 1 from a cell) 


\section{WO: write operation (writing a 0 to a cell)}

W1: write operation (writing a 1 to a cell)

The response will be 0 or 1 if the test algorithm reads a cell, and they are specified as R0 and $\mathrm{R} 1$, respectively. Similarly, writing a 1 into a cell is denoted as W1 and writing 0 as W0.

A March- based test algorithm is a fixed sequence of read and write operations called March element. It is specified by a number of reads and write operations and $\mathrm{n}$ address order. MATS, MATS+, March-C, March-Y, March-A, and March-B are different types of March- based tests. Because of its simplicity and high fault coverage in most contemporary memory BIST, March based test algorithm is implemented because of its high fault coverage and simplicity. The various March algorithms and Features are stated in the below table:

Table 1. Memory Algorithms and its features.

\begin{tabular}{|c|c|c|}
\hline SI. No & $\begin{array}{l}\text { Type of the March } \\
\text { algorithm }\end{array}$ & Features \\
\hline 1. & MARCHING $1 / 0$ Test & $\begin{array}{l}\text { It can detect Auxiliary faults (AF) and Stuck at faults (SAF) and Transition faults } \\
\text { (TF). }\end{array}$ \\
\hline 2. & MATS Test & Modified Algorithmic Test Sequence, it can detect OR type technology. \\
\hline 3. & MATS+ Test & It can detect all Stuck at faults (SAF) and Auxiliary faults (AF). \\
\hline 4. & MATS++ & It is like MAT+ additionally it covers transition faults (TF). \\
\hline 5. & MARCHX & $\begin{array}{l}\text { It can detect all stuck at faults, auxiliary faults, transition faults and Coupling } \\
\text { faults }\end{array}$ \\
\hline 6. & MARCH C & $\begin{array}{l}\text { It can also detect all stuck at fault, auxiliary faults, transition faults and Coupling } \\
\text { faults }\end{array}$ \\
\hline 7. & MARCH C- & Redundancy of MARCH C algorithm is removed. \\
\hline 8. & MARCH A & $\begin{array}{l}\text { It can detect AF's, SAF's, linked Coupling Fault CFid's, TF's and certain CFin's } \\
\text { linked with CFid's }\end{array}$ \\
\hline 9. & MARCH Y & Extended version of MARCHX \\
\hline 10. & MARCH B & Extended version of MARCHA \\
\hline
\end{tabular}

\section{BUIILT -IN-SELF-TEST(BIST)}

Built-In Self-Test (BIST), test generation and response evaluation hardware are included on-chip so that in-circuit tests can be performed with minimal need for external test equipment, if any. The BIST technique is a common technique to test memories (RAM and ROMs).

There are two types of BIST; On-line BIST and Off-line BIST. 
a. On-line BIST: It is implemented on the chip itself. It has area overhead but has the shortest test time.

b. Off-line BIST: It is implemented off the chip itself. It has no area overhead but has the longest test time.

\section{TEST ARCHITECTURE}

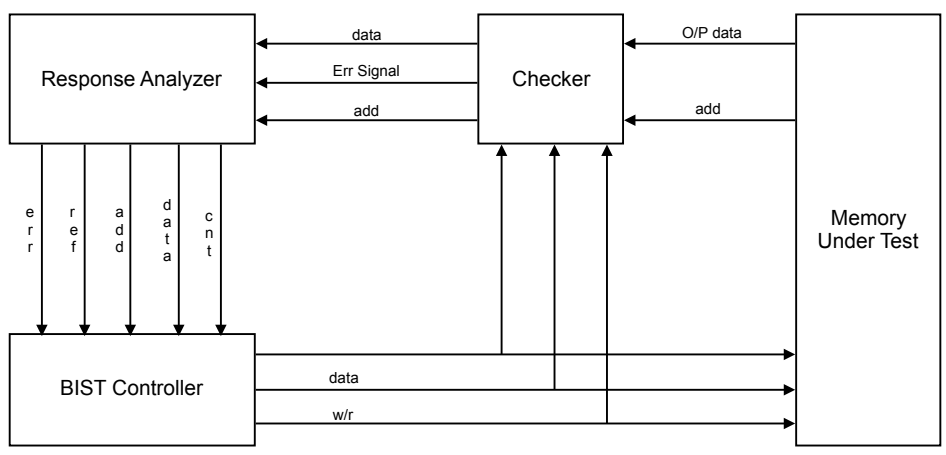

Figure 1. BIST Architecture.

\section{EXPLANATION AND RESULTS}

Built-In Self-Test (BIST), test generation and response evaluation hardware are included on-chip so that in-circuit tests can be performed with minimal need for external test equipment, if any. The BIST technique is a common technique to test memories (RAM and $\mathrm{ROMs}$ ).

This architecture consists of BIST Controller, Memory Under Test (MUT), Checker and Response Analyzer. Clock signal becomes to enable the BIST controller to starts working. The BIST controller gives the control signal to the memory. Then the memory undergoes read or/write operation according to the March algorithm.

Then the output from the memory is given to the checker. The checker compares the output from the memory to the data stored inside it. Whenever the fault occurs the checker gives the error signal, the original data along with the address to the response analyzer. 
The response analyzer is used to switch the controller from normal to repair mode. Whenever the repair mode becomes to enable the controller automatically enables the write signal to repair the fault according to the address and data given by the response analyzer. After the repair operation gets completed the ref signal becomes enable to indicate that fault is repaired. As the continue signal becomes to enable the controller switches to normal mode. This process is continued until the end of the operation. The export mask address signal is used to indicate whether the fault is repairable or not. In this way, this test architecture is used to test and repair the fault in memory with maximum accuracy.

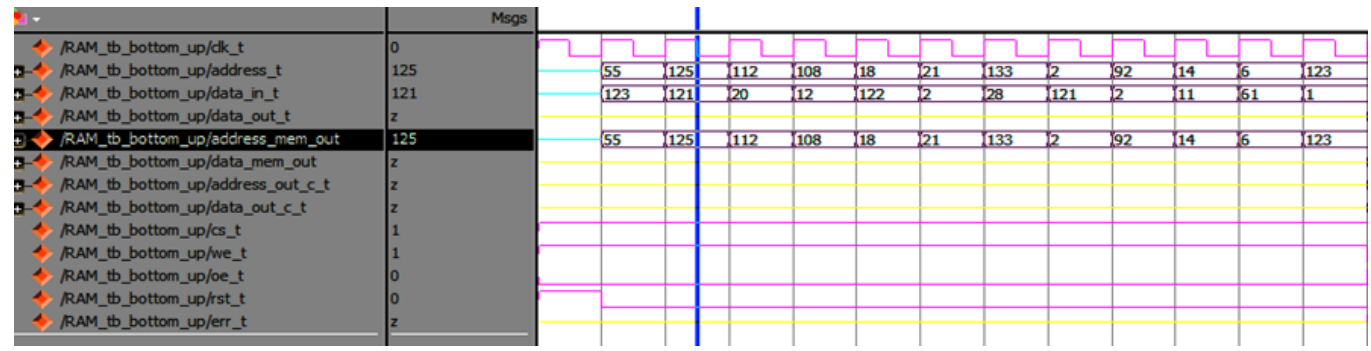

Figure 2. Data writing an operation into the memory and checker.

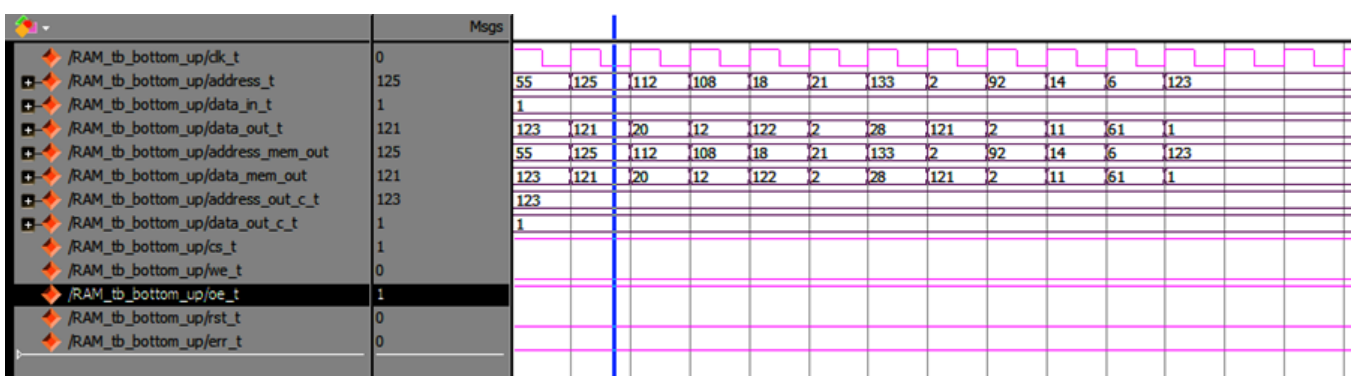

Figure 3. Data reading operation from the memory and it is compared inside the checker for error.

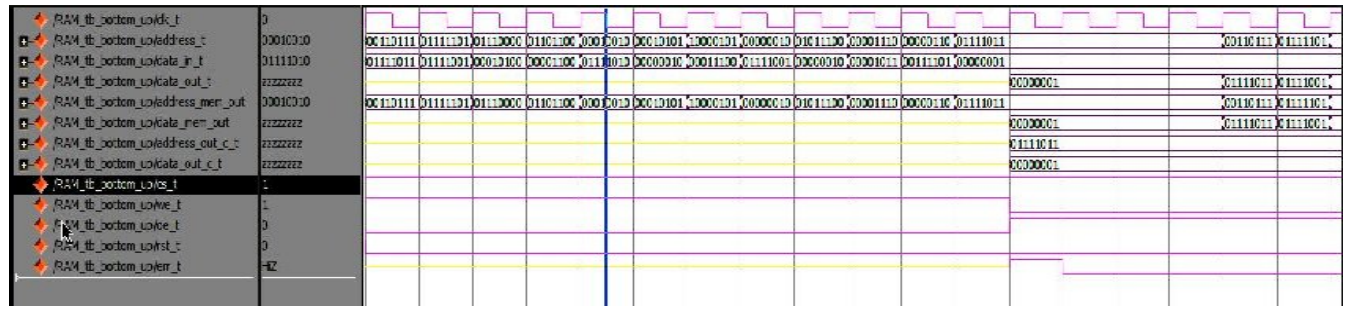

Figure 4. Reading, Writing, and comparison of the data inside the checker for detecting the error. 


\section{CONCLUSION}

MARCH tests are extensively being used today for functional testing of memory technologies. They are more efficient with better fault coverage than the older classical pattern. In this project modified MARCH C Algorithm with modified BIST architecture are proposed. With this simple BIST architecture and modified MARCH C, testing time can be reduced because two read/write operations carried out in a single clock time. So, Testing Speed can also be doubled. It also provides better fault coverage as MARCH C algorithm covers most of the faults in the memory.

\section{ACKNOWLEDGMENT}

We thank the ECE department of Kalasalingam Academy of Research and education, Krishnankoil for supporting this research by providing their center for VLSI lab facility. The Facility which is sponsored by Department of Science and technology (DST) under Fund for Improvement of S\&T Infrastructure (FIST) Scheme. 


\section{REFERENCES}

Adams, R. D. (2003). High Performance Memory Testing: Design Principles, Fault Modeling and SelfTest. Springer US. https://doi.org/10.1007/b101876

Begum, I., Khayyum, S., \& Ravinder, K. (2013). A Built-In Self-Test By Enhanced Faults Coverage with Microcode Optimization for Embedded Memory. International fournal of Advanced Research in Computer and Communication Engineering, 2(7). https://ijarcce.com/ upload/2013/july/42-h-Khayyum\%20Shaik-A\%20Built-In\%20Self\%20Test\%20 By $\% 20$ Enhanced $\% 20$ Faults $\% 20$ Coverage $\% 20$ with $\% 20$ Microcode.pdf

Gockburn, B. F. (1994). Tutorial on Semiconductor Memory Testing. Fournal of Electronic Testing: Theory and Applications, 5(4), 321-336. https://doi.org/10.1007/BF00972517

Hamdioui, S., Al-Ars, Z., \& Van de Goor, A. J. (2002). Testing Static and Dynamic Faults in Random Access Memories. In Proceedings of 20th IEEE VLSI Test Symposium, pp. 395-400, Monterey, CA, USA. https://doi.org/10.1109/VTS.2002.1011170

Hamdioui, S., Van de Goor, A. J., \& Rodgers, M. (2002). March SS: A Test for All Static Simple RAM Faults. In Proceedings of 2002 IEEE International Workshop on Memory Technology, Design, and Testing, pp. 95-100, Bendor, France. https://doi.org/10.1109/ MTDT.2002.1029769

Hamdioui, S., Wadsworth, R., De los Reyes, J., \& Van de Goor, A. J. (2003). Importance of Dynamic Faults for New SRAM Technologies. In 8th IEEE Proceedings Of European Test Workshop, pp. 29-34, Maastricht, Netherlands, Netherlands. https://doi. org/10.1109/ETW.2003.1231665

Kapse, V., \& Arif, M. (2012). Optimization of Microcode Built-In Self-Test by Enhanced Faults Coverage for Embedded Memory. IEEE Students' Conference on Electrical, Electronics and Computer Science, Bhopal, India. https://doi.org/10.1109/SCEECS.2012.6184782

Li,J. F., Cheng, K. L., Huang, C. T., \& Wu, C. W. (2001). March-based RAM diagnostic algorithms for stuck-at and coupling faults. Proceedings International Test Conference 2001 (Cat. No.01CH37260), Baltimore, MD, USA, pp. 758-767. https://doi.org/10.1109/ TEST.2001.966697 
Parvathi, M., Vasantha, N., \& Prasad, K. S. (2012). Modified March C Algorithm for Embedded Memory Testing. International fournal of Electrical and Computer Engineering (IFECE), 2(5), 571-576. https://pdfs.semanticscholar.org/ a697/29ca02295d535faf06141b7c9572bfc48e86.pdf

Sharma R. K., \& Sood, A. (2010). Modeling and Simulation of Multi-operation Microcode-Based Built-In Self Test for Memory Fault Detection and Repair. IEEE Computer Society Annual Symposium on VLSI. https:/ / doi.org/ 10.1109/ISVLSI.2010.88

Singh, S., \& Chandrawad, U. (2013). Built-In-Self Test for Embedded Memories by Finite State Machine. International Journal of Digital Application $\mathcal{E}$ Contemporary research, 2(2). http://ijdacr.com/uploads/papers/Swati+singh_paper.pdf

Tanabe, A., Takeshima, T., Koike, H., Aimoto, Y., Takada, M., Ishijima, T., ... Okuda, T. (1992). A 30-ns 64 Mb DRAM with built-in self-test and selfrepair function. IEEE Journal of Solid-State Circuits, 27(11), 1525-1533. https://doi. org/10.1109/4.165332

Tehranipour, M. H., Nourani, M., Fakhraie, S. M., Movahedin, M. R., \& Navabi, Z. (2011). Embedded Test for Processor and Memory Cores in System-on-Chips. https://wenku. baidu.com/view/260a9369561252d380eb6ee3.html

Van de Goor, A. J. (1998). Testing Semiconductor Memories, Theory and Practice. Netherlands: ComTex Publishing. https://doi.org/10.1002/qre.4680080216

Van de Goor, A.J., \& Al-Ars, Z. (2000). Functional memory faults: a formal notation and a taxonomy. In Proceedings of 18 th IEEE VLSI Test Symposium, Montreal, Quebec, Canada, Canada. https://doi.org/10.1109/VTEST.2000.843856

Wang, D., Hu, Y., Li, H. W., \& Li, X. W. (2008). The Design- for-Testability Features and Test Implementation of a Giga Hertz General Purpose Microprocessor. Fournal of computer science and technology, 23(6), 1037-1046. http://jcst.ict.ac.cn/EN/Y2008/ $\mathrm{V} 23 / \mathrm{I} 6 \% 20 \% 20 \% 20 \% 20 \% 20 \% 20 \% 20 \% 20 \% 20 / 1037$ 
Zakaria, N. A., Hassan, W. Z. W., Halin, I. A., Sidek, R. M., \& Xiaoqing, W. (2012). Fault detection with optimum march test algorithm. Third International Conference on Intelligent Systems Modelling and Simulation. https:// doi.org/10.1109/ISMS.2012.88 


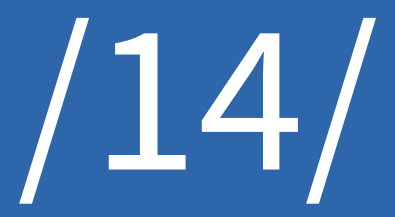




\section{QUEUING SYSTEM IN SYNCHRONOUS OPTICAL NETWORK (SONET)}

S. Maragathasundari

Associate professor, Department of Mathematics, Kalasalingam Academy of Research and Education.

Krishnankoil, (India). E-mail: maragatham01@gmail.com ORCID: https://orcid.org/0000-0003-1210-6411

\section{P. Suthersan}

Assistant professor, Department of Mathematics, Kalasalingam Academy of Research and Education. Krishnankoil, (India). E-mail: suthersan.p@klu.ac.in ORCID: http://orcid.org/0000-0003-0712-0518

K. S. Dhanalakshmi

Assistant professor, Department of Electronics and Communication Engineering, Kalasalingam Academy of Research and Education.

Krishnankoil, (India).

E-mail: k.s.dhanalakshmi@klu.ac.in ORCID: https://orcid.org/0000-0001-6285-3656

\section{Citación sugerida:}

Maragathasundari, S., Suthersan, P., y Dhanalakshmi, K. S. (2020). Queuing system in Synchronous Optical Network (SONET). 3C Tecnología. Glosas de innovación aplicadas a la pyme. Edición Especial, Marzo 2020, 231-245. http://doi.org/10.17993/3ctecno.2020.specialissue4.231-245

\section{Suggested citation:}

Maragathasundari, S., Suthersan, P., \& Dhanalakshmi, K. S. (2020). Queuing system in Synchronous Optical Network (SONET). 3C Tecnología. Glosas de innovación aplicadas a la pyme. Edición Especial, Marzo 2020, 231-245. http://doi.org/10.17993/3ctecno.2020.specialissue4.231-245 


\section{ABSTRACT}

This paper researches an investigation on Queuing framework in Synchronous Optical Network (SONET). Optical fiber utilized in SONET can blame on various conditions that are capricious, which is a fundamental dependability worry for power lattice interchanges. Different transmission advances have been utilized in whole deal interchanges, for example, optical fiber, microwave, or satellite. Optical fiber can blame on various flighty conditions which make it a noteworthy danger to arrange unwavering quality. Phases of administration in SONET, administration intrusion in this system are well explained. The Queuing issue occurring in this system is very much tackled by strengthening variable methodology and the comparing line execution measures are inferred. The purpose of issue emerged is very much anticipated by this Queuing approach and the administration interference could be limited or to a NIL base. Numerical delineation encourages the model to be defended to an incredible extent. Graphical portrayal unmistakably clarifies the presentation proportions of the Queuing framework in SONET.

\section{KEYWORDS}

Batch arrival, Optional First Stage, Compulsory Second Stage, Service Interruption. 


\section{INTRODUCTION}

SONET is utilized to change over electrical sign into optical sign so it can travel longer separations. Synchronous Optical NET work (SONET) is a typical for optical televise communications transport, which was created in the mid-1980s, and stays in boundless use today. Contrasted with Ethernet cabling that traverses separations up to100 meters, SONET fiber ordinarily runs a lot further. Indeed, even short achieve connections range as much as 2 kilometers (1.2 miles); intermediate and long achieve connections spread many kilometers. Along these lines it is appropriate for whole deal transmission, for example, the one in the power lattice correspondences. Wu, Kobrinski, Ghosal, and Lakshman (1994) examined a few DCS design upgrade choices, including a parallel handling/crossinterface DCS engineering, which may improve the administration rebuilding time. Boehm, Ching, Griffith, and Saal (1986) gave an account of the exercises in different benchmarks associations, with accentuation on a synchronous system proposition which is as of now being talked about in the $\mathrm{T} 1$ advisory group. Way, Smith, Johnson, and Izadpanah (1992) tentatively confirmed the system idea and talked about various system applications for bursty information traffic and persistent voice/video traffic. Blumenthal et al. (2003) explored the sign handling procedures, Hac and Mutlu (1989) researched the B-ISDN convention, guidelines utilized in the Broadband reference model. Lee, Sherali, Han, and Kim (2000) dealt with a system plan issue emerging from the sending of synchronous optical systems (SONET), a standard of transmission utilizing optical fiber innovation. Cosares, Deutsch, Saniee, and Wasem (1995) inspected SONET framework by the Bellcore customer organizations has spared 10 to 30 percent in expenses and requests of greatness in time. Chao, Shtirmer, and Smoot (1989) broke down the physical layer of the system utilizes the synchronous optical system transmission design. Fundamental ideas are talked about and reviewed by Jue, Yang, Kim, and Zhang (2009). Kang, Park, Shin, and Jeong (1995) watched the normal for the system relying on the collected transmission limit of the network. Maragathasundari and Balamurugan (2015) contemplated the presentation examination of bunch landing line with two phases of administration. Maragathasundari and Dhanalakshmi (2018) investigated versatile adhoc systems issue A Queuing approach. Maragathasundari and Srinivasan (2012) made an investigation on M/G/1 input line with three phase and different server get-away. Maragathasundari and Srinivasan (2015) examined a Non-Markovian Multistage Batch entry line with breakdown and reneging. An examination on the investigation of execution proportion of mass information line with $\mathrm{N}$ 
sort of extra discretionary administration, administration interference and deterministic get-away were inspected by Maragathasundari and Sowmiah (2016).

\subsection{ADVANTAGES OF SONET}

1) Transmits data to large distances.

2) Low electromagnetic interference.

3) High data rates.

4) Large Bandwidth.

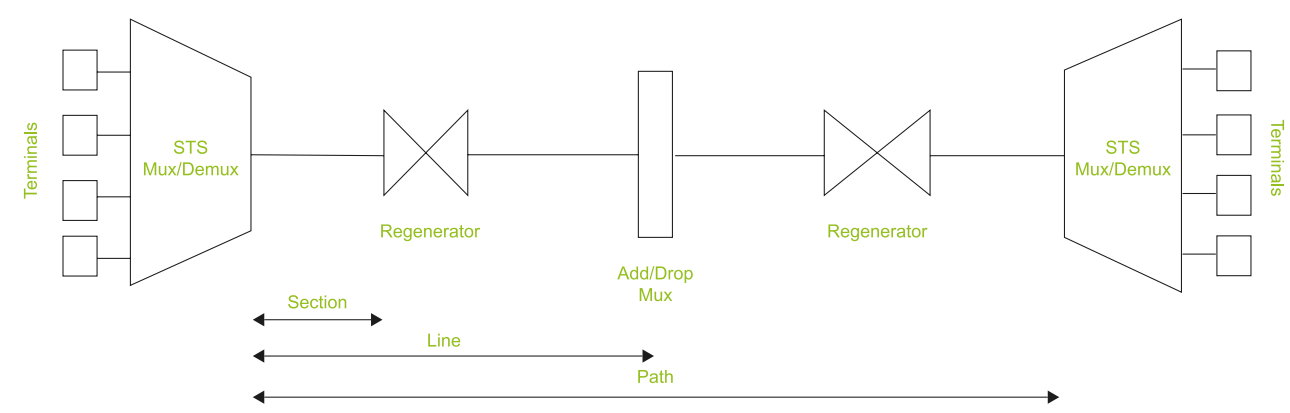

Graphic 1. SONET Network Elements.

\subsection{SONET CONNECTIONS}

1) Section: Portion of system interfacing two neighboring gadgets.

2) Line: Portion of system interfacing two neighboring multiplexers.

3) Path: End-to-end segment of the system.

\section{*STS Multiplexer:}

Performs multiplexing of sign.

\section{*STS Demultiplexer:}

Performs demultiplexing of sign.

Converts optical sign to electrical sign.

\section{*Regenerator:}

It is a repeater, that takes an optical sign and recovers (builds the quality) it. 


\section{*Add/Drop Multiplexer:}

It permits including sign originating from various sources into a given way or expelling a sign.

\subsection{SONET LAYERS}
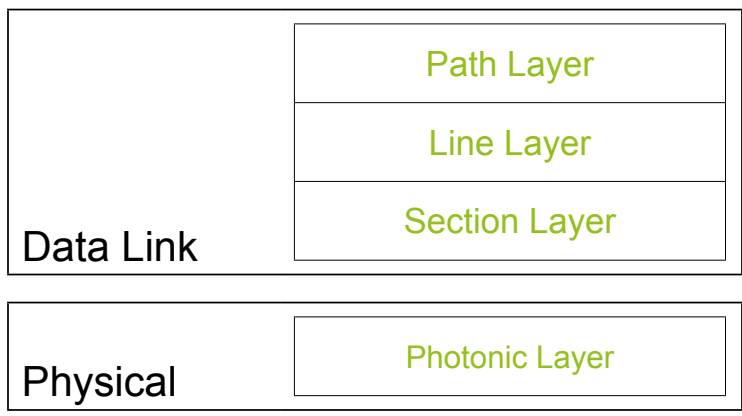

Graphic 2. SONET Layers.

SONET includes four functional layers:

\section{1) Path Layer:}

a. It is in charge of the development of sign from its optical source to its optical goal.

b. STS Mux/Demux gives way layer capacities.

\section{2) Line Layer:}

a. It is in charge of the development of sign over a physical line.

b. STS Mux/Demux and Add/Drop Mux give Line layer capacities.

\section{3) Section Layer:}

a. It is in charge of the development of sign over a physical area.

b. Each gadget of system gives segment layer capacities.

\section{4) Photonic Layer:}

a. It relates to the physical layer of the OSI model.

b. It incorporates physical determinations for the optical fiber channel (nearness of light $=1$ and nonappearance of light $=0$ ). 


\subsection{PERFORMANCE REQUIREMENTS}

We assume the following to describe the queuing model of our study.

1) Batch arrival - Queue - We consider a solitary server line which will give two distinct administrations, an Essential Service and Optional Service.

2) Essential administration - 2 phases: One of the benefits of SONET is that it can pass on gigantic payloads (more than $50 \mathrm{Mbps}$ ). To achieve this capacity, the STS SPE can be sub-apportioned into more diminutive sections or structures, known as VTs (Virtual tributaries)

*Optional First Stage: Except for connected sign, all data sources are at last changed over to a base setup of a synchronous STS-1 signal (51.84 Mbps or higher). Lowerspeed information sources, for instance, DS-1s are first piece or byte-multiplexed into VTs. Several synchronous STS-1s are then multiplexed together in either a singular or two-mastermind system to outline an electrical STS $-\mathrm{N}$ signal $(\mathrm{N}>=1)$.

* Compulsory Second Stage: Any kind of organization, running from voice to quick data and video, can be recognized by various types of organization connectors. An organization connector maps the sign into the payload envelope of the STS-1 or VT. New organizations and sign can be transported by including new organization connectors at the edge of the SONET sort out.

3) Optional administration - Service Interruption happens - Optical Cable Failures are considered here as Service Interruption during this Optional Service.

Three kinds of optical strands have been utilized in the whole deal transport of information.

- Buried fiber optic links have a higher disappointment rate than the two overhead links.

- Optical ground wire links and introduced overhead on posts or transmission towers.

- All dielectric self-supporting cables introduced overhead on posts or transmission towers. 
Two sorts of disappointments are being considered,

(a) "cable cut" disappointments which will influence both the working strand and the assurance strand, and

(b) "Strand disappointments" which will bomb just one strand inside the link.

4) Completion of Both administrations- Dissatisfied Customers (not ready to utilize the multiplexing procedure successfully) can join the tail of the first line to get a Feedback administration.

\section{MATHEMATICAL PORTRAYAL OF THE QUEUING MODEL}

The arithmetical portrayal of the Queuing frame work has the option to be described by the resulting proposition:

Customers meet up at the structure in clusters of variable size in a compound strategy pursues Poisson conveyance. Let $\lambda d_{j} d t(j=1,2,3 \ldots)$ be the first order probability that a batch of $j$ customers arrives at the system during a short duration of time $(t, t+d t)$ where $0 \leq d_{j} \leq 1$ and $\sum_{j=1}^{n} d_{j}=1$ and $\lambda>0$ is the mean landing rate of the batches.

The administration time pursues general(arbitrary) circulation. First stage of essential service follows distribution function as $L_{e_{1}}(x)$ and density function $l_{e_{1}}(x)$. Let $\mu_{\left(e_{1}\right)}(x) d x$ be the conditional density function. Hence, we have:

$$
\mu_{\left(e_{1}\right)}(x)=\frac{l_{e_{1}}(x)}{1-L_{e_{1}}(x)}, \quad l_{e_{1}}(x)=\mu_{\left(e_{1}\right)}(x) e^{-\int_{0}^{s} \mu_{\left(e_{1}\right)}(s) d s}
$$

For second stage of essential service,

$$
\mu_{\left(e_{2}\right)}(x)=\frac{l_{e_{2}}(x)}{1-L_{e_{2}}(x)} \quad, l_{e_{2}}(x)=\mu_{\left(e_{2}\right)}(x) e^{-\int_{0}^{s} \mu_{\left(e_{2}\right)}(s) d s}
$$

For optional service,

$$
\mu_{k}(x)=\frac{l_{k}(x)}{1-L_{k}(x)} \quad, l_{k}(x)=\mu_{k}(x) e^{-\int_{0}^{s} \mu_{k}(s) d s}
$$


Service interruption follows Poisson distribution with mean rate $\beta>0$.

\section{GOVERNING EQUATIONS OF THE MODEL}

$$
\begin{gathered}
\frac{d}{d x} P_{n}^{\left(e_{1}\right)}(x)+\left(\lambda+\mu_{\left(e_{1}\right)}(x)\right) P_{n}^{\left(e_{1}\right)}(x)=\lambda \sum_{j=1}^{n} d_{j} P_{n-j}^{\left(e_{1}\right)}(x) \\
\frac{d}{d x} P_{0}^{\left(e_{1}\right)}(x)+\left(\lambda+\mu_{\left(e_{1}\right)}(x)\right) P_{0}^{\left(e_{1}\right)}(x)=0 \\
\frac{d}{d x} P_{n}^{\left(e_{2}\right)}(x)+\left(\lambda+\mu_{\left(e_{2}\right)}(x)\right) P_{n}^{\left(e_{2}\right)}(x)=\lambda \sum_{j=1}^{n} d_{j} P_{n-j}^{\left(e_{2}\right)}(x) \\
\frac{d}{d x} P_{0}^{\left(e_{2}\right)}(x)+\left(\lambda+\mu_{\left(e_{2}\right)}(x)\right) P_{0}^{\left(e_{2}\right)}(x)=0 \\
\frac{d}{d x} K_{n}(x)+\left(\lambda+\mu_{k}(x)+\beta\right) K_{n}(x)=\lambda \sum_{j=1}^{n} d_{j} K_{n-j}(x) \\
\frac{d}{d x} K_{0}(x)+\left(\lambda+\mu_{k}(x)+\beta\right) K_{0}(x)=0 \\
\frac{d}{d x} M_{n}(x)+\left(\lambda+\mu_{m}(x)\right) M_{n}(x)=\lambda \sum_{j=1}^{n} d_{j} M_{n-j}(x) \\
\frac{d}{d x} M_{0}(x)+\left(\lambda+\mu_{m}(x)\right) M_{n}(x)=0 \\
\lambda Q=\int_{0}^{\infty} M_{0}(x) \mu_{m}(x) d x+(1-m) \int_{0}^{\infty} P_{0}^{\left(e_{2}\right)}(x) \mu_{\left(e_{2}\right)}(x) d x+(1-r) Q \int_{0}^{\infty} K_{0}(x) \mu_{k}(x)
\end{gathered}
$$

\section{BOUNDARY CONDITIONS}

The following boundary conditions are used to solve the above equations:

$$
\begin{gathered}
P_{n}^{\left(e_{1}\right)}(0)=\int_{0}^{\infty} M_{n+1}(x) \mu_{m}(x) d x+(1-m) \int_{0}^{\infty} P_{n+1}^{\left(e_{2}\right)}(x) \mu_{\left(e_{2}\right)}(x) d x+r \int_{0}^{\infty} K_{n}(x) \mu_{k}(x) d x+\lambda D_{n+1} \mathrm{Q} \\
P_{n}^{\left(e_{2}\right)}(0)=\int_{0}^{\infty} P_{n}^{\left(e_{1}\right)}(x) \mu_{\left(e_{1}\right)}(x) d x \\
K_{n}(0)=\int_{0}^{\infty} P_{n}^{\left(e_{2}\right)}(x) \mu_{\left(e_{2}\right)} d x \\
M_{n}(0)=\beta \int_{0}^{\infty} K_{n}(x)
\end{gathered}
$$




\section{QUEUE LENGTH DISTRIBUTION}

\section{Usage of Supplementary variable technique}

We multiply (1) by $z^{n}$ and sum over $\mathrm{n}$ from 1 to $\infty$ and add it to (2).

We get,

$$
\frac{d}{d x} P^{\left(e_{1}\right)}(x, z)+\left(\lambda-\lambda D(z)+\mu_{\left(e_{1}\right)}(x)\right) P^{\left(e_{1}\right)}(x, z)=0
$$

Again integrating the above from 0 to $\mathrm{n}$, we get

$$
P^{\left(e_{1}\right)}(x, z)=P^{\left(e_{1}\right)}(0, z) e^{-(\lambda-\lambda D(z)) x} \int_{0}^{x} \mu_{\left(e_{1}\right)}(t) d t
$$

Again integrating $\left(^{*}\right)$ by parts with respect to $x$ yields,

$$
P^{\left(e_{1}\right)}(z)=P^{\left(e_{1}\right)}(0, z)\left[\frac{1-L_{\left(e_{1}\right)}(\lambda-\lambda D(z))}{(\lambda-\lambda D(z))}\right]
$$

Multiplying both sides of the $\left.{ }^{*}\right)$ by $\mu_{\left(e_{1}\right)}(x)$ and integrating over $x$, we get:

$$
\int_{0}^{\infty} P^{\left(e_{1}\right)}(x, z) \mu_{\left(e_{1}\right)}(x)=P^{\left(e_{1}\right)}(0, z) L_{\left(e_{1}\right)}(\lambda-\lambda D(z))
$$

Applying the same concept for the second stage (optional) in essential service $\left[P_{n}^{\left(e_{1}\right)}(x)\right]$, optional service $\left[K_{n}(x)\right]$, and repair process $\left[M_{n}(x)\right]$, we get,

$$
\begin{gathered}
\text { i) } P^{\left(e_{2}\right)}(z)=P^{\left(e_{2}\right)}(0, z)\left[\frac{1-L_{\left(e_{2}\right)}(\lambda-\lambda D(z))}{(\lambda-\lambda D(z))}\right] \\
=P^{\left(e_{1}\right)}(0, z) L_{\left(e_{1}\right)}(\lambda-\lambda D(z))\left[\frac{1-L_{\left(e_{2}\right)}(\lambda-\lambda D(z))}{(\lambda-\lambda D(z))}\right]
\end{gathered}
$$

Also we have,

$$
\begin{array}{r}
\int_{0}^{\infty} P^{\left(e_{2}\right)}(x, z) \mu_{\left(e_{2}\right)}(x) d x=P^{\left(e_{1}\right)}(0, z) L_{\left(e_{1}\right)}(\lambda-\lambda D(z)) L_{\left(e_{2}\right)}(\lambda-\lambda D(z)) \\
\text { ii) } K(z)=K(0, z)\left[\frac{1-L_{k}(\lambda-\lambda D(z)+\beta)}{(\lambda-\lambda D(z)+\beta)}\right]=P^{\left(e_{1}\right)}(0, z) L_{\left(e_{1}\right)}(\lambda-\lambda D(z))
\end{array}
$$




$$
L_{\left(e_{2}\right)}(\lambda-\lambda D(z))\left[\frac{1-L_{\left(e_{2}\right)}(\lambda-\lambda D(z))}{(\lambda-\lambda D(z))}\right]
$$

$\int_{0}^{\infty} K(x, z) \mu_{k}(x) d x=P^{\left(e_{1}\right)}(0, z) L_{\left(e_{1}\right)}(\lambda-\lambda D(z)) L_{\left(e_{2}\right)}(\lambda-\lambda D(z)) L_{k}(\lambda-\lambda D(z)+\beta)$

$M(z)=\beta z P^{\left(e_{1}\right)}(0, z) L_{\left(e_{1}\right)}(\lambda-\lambda D(z)) L_{\left(e_{2}\right)}(\lambda-\lambda D(z))\left[\frac{1-L_{k}(\lambda-\lambda D(z)+\beta)}{(\lambda-\lambda D(z)+\beta)}\right]\left[\frac{1-H(\lambda-\lambda D(z))}{(\lambda-\lambda D(z))}\right]$

$\int_{0}^{\infty} M(x, z) \mu_{m}(x) d x=\beta z^{\left(e_{1}\right)}(0, z) L_{\left(e_{1}\right)}(\lambda-\lambda D(z)) L_{\left(e_{2}\right)}(\lambda-\lambda D(z))\left[\frac{1-L_{k}(\lambda-\lambda D(z)+\beta)}{(\lambda-\lambda D(z)+\beta)}\right] H(\lambda-\lambda D(z))$

Now $\sum_{n=0}^{\infty} z^{n}(10)$, using (9) and further using (18), (20), (22) we get,

$$
P^{\left(e_{1}\right)}(0, z)=\frac{\lambda Q(D(z)-1)}{z-L_{\left(e_{1}\right)}(a) L_{\left(e_{2}\right)}(a)\left[\beta z H(a)\left[\frac{1-L_{k}(b)}{b}\right]+(1-m)+r L_{k}(b)\right]}
$$

Substituting (23) in (15), (17), (19), (21) we get,

$$
\begin{aligned}
& P^{\left(e_{1}\right)}(z)=\frac{\left[L_{\left(e_{1}\right)}(a)-1\right] Q}{z-L_{\left(e_{1}\right)}(a) L_{\left(e_{2}\right)}(a)\left[\beta z H(a)\left[\frac{1-L_{k}(b)}{b}\right]+(1-m)+r L_{k}(b)\right]} \\
& P^{\left(e_{2}\right)}(z)=\frac{L_{\left(e_{1}\right)}(a)\left[L_{\left(e_{2}\right)}(a)-1\right] Q}{z-L_{\left(e_{1}\right)}(a) L_{\left(e_{2}\right)}(a)\left[\beta z H(a)\left[\frac{1-L_{k}(b)}{b}\right]+(1-m)+r L_{k}(b)\right]} \\
& K(z)=\frac{a L_{\left(e_{1}\right)}(a) L_{\left(e_{2}\right)}(a)\left[L_{k}(b)-1\right]}{b\left[z^{\left.\left.\left.-L_{(}\right)^{(a) L_{(}}\right)^{(a)}\left[\beta z H(a)\left[\frac{-L_{k}(b)}{b}\right]+(1-m)+r L_{k}(b)\right]\right]}\right.} \\
& M(z)=\frac{\beta z L_{\left(e_{1}\right)}(a) L_{\left(e_{2}\right)}(a)\left[L_{k}(b)-1\right][1-H(a)]}{b\left[z-L_{\left(e_{1}\right)}(a) L_{\left(e_{2}\right)}(a)\left[\beta z H(a)\left[\frac{1-L_{k}(b)}{b}\right]+(1-m)+r L_{k}(b)\right]\right]}
\end{aligned}
$$

Where, $a=\lambda-\lambda D(z), b=\lambda-\lambda D(z)+\beta$

\section{PROBABILITY CAPACITY FUNCTION OF THE QUEUE LENGTH}

Let $J_{q}(z)$ be the PGFof the queue length

$$
J_{q}(z)=P^{\left(e_{1}\right)}(z)+P^{\left(e_{2}\right)}(z)+K(z)+M(z)
$$

Adding (24) to (27), we get, 


$$
J_{q}(z)=\frac{Q\left\{b\left[L_{\left(e_{1}\right)}(a) L_{\left(e_{2}\right)}(a)-1\right]+L_{\left(e_{1}\right)}(a) L_{\left(e_{2}\right)}(a)\left[L_{k}(b)-1\right][a+\beta z[1-H(a)]]\right\}}{b\left[z-L_{\left(e_{1}\right)}(a) L_{\left(e_{2}\right)}(a)\left[\beta z H(a)\left[\frac{1-L_{k}(b)}{b}\right]+(1-m)+r L_{k}(b)\right]\right]}
$$

\section{IDLE TIME AND UTILIZATION FACTOR}

Idle time is determined using the condition:

$$
J_{q}(1)+Q=1
$$

Applying LH rule we get,

$$
\begin{aligned}
& J_{q}(z)=\frac{N^{\prime}(1)}{D^{\prime}(1)} \\
& Q=\frac{D^{\prime}(1)}{D^{\prime}(1)+N^{\prime}(1)}
\end{aligned}
$$

From the idle factor $Q$, the utilization rate is calculated.

To find $L_{q}$ the length of the Queue and the Queue performance measures.

We have $L_{q}(z)=\frac{d}{d z} J_{q}(z) \mid z=1=\frac{0}{0}$ (indeterminate form)

$$
L_{q}=\lim _{z \rightarrow 1} \frac{D^{\prime}(z) N^{\prime \prime}(z)-N^{\prime}(z) D^{\prime \prime}(z)}{2\left(D^{\prime}(z)\right)^{2}}
$$

Here $J_{q}(z)=\frac{N(z)}{D(z)}$ where $\mathrm{N}(\mathrm{z})$ and $\mathrm{D}(\mathrm{z})$ are the numerator and denominator of (28).

$$
\begin{gathered}
N^{\prime}(1)=\lambda E(I)\left[E\left(L_{\left(e_{1}\right)}\right)+E\left(L_{\left(\left(_{2}\right)\right.}\right)+1+\beta E(H)\left(L_{k}(\beta)-1\right)\right] \\
N^{\prime \prime}(1)=2(-\lambda E(I))^{2}\left[E\left(L_{\left(e_{1}\right)}\right)+E\left(L_{\left(e_{2}\right)}\right)\right] \\
+\beta(\lambda E(I))^{2}\left[E\left(L_{\left(e_{1}\right)}{ }^{2}\right)+E\left(L_{\left(e_{2}\right)}{ }^{2}\right)+2\left[E\left(L_{\left(e_{1}\right)}\right) E\left(L_{\left(\left(_{2}\right)\right.}\right)\right]\right](-\lambda E(I))^{2}\left[-E(H)\left[E\left(L_{\left(\left(_{1}\right)\right.}\right)+E\left(L_{\left(\left(_{2}\right)\right.}\right)+L_{k}^{\prime}-\beta\right]\right. \\
\left.+\beta E(H)\left(L_{k}(\beta)+(-\lambda E(I))\left(E\left(L_{\left(e_{1}\right)}\right)+E\left(L_{\left(\left(_{2}\right)\right.}\right)+L_{k^{\prime}}\right)\right)+(-\lambda E(I))^{2} \beta E\left(H^{2}\right) L_{k}(\beta)\right] \\
D^{\prime}(1)=(-\lambda E(I))\left[1-\left(1-L_{k}(\beta)+(1-m)+r L_{k}(\beta)\right)\right] \\
+\beta\left\{1+(-\lambda E(I))\left[E\left(L_{\left(e_{1}\right)}\right)+E\left(L_{\left(\left(_{2}\right)\right.}\right)\right]\left[\left(1-L_{k}(\beta)\right)+(1-m)+r L_{k}(\beta)\right]\right. \\
\left.-\left[\left(1-L_{k}(\beta)\right)+\lambda E(I) E(H)\left(1-L_{k}(\beta)\right)+\beta \lambda E(I) L_{k}^{\prime}+r L_{k}^{\prime} \lambda E(I)\right]\right\}
\end{gathered}
$$




$$
\begin{aligned}
D^{\prime \prime}(1)= & -\lambda E(1) 2\left\{1+(-\lambda E(I))\left[E\left(L_{\left(e_{1}\right)}\right)+E\left(L_{\left(e_{2}\right)}\right)\right]\left[\left(1-L_{k}(\beta)\right)+(1-m)+r L_{k}(\beta)\right]\right. \\
- & {\left.\left[\left(1-L_{k}(\beta)\right]+\lambda E(I) E(H)\left(1-L_{k}(\beta)\right)+\beta \lambda E(I) L^{\prime}{ }_{k}+r L_{k}{ }_{k} \lambda E(I)\right]\right\} } \\
+ & \beta\left\{-(-\lambda E(I))^{2}\left(E\left(L_{\left(e_{1}\right)}{ }^{2}\right)+E\left(L_{\left(e_{2}\right)}{ }^{2}\right)+2 E\left(L_{\left(e_{1}\right)}\right) E\left(L_{\left(e_{2}\right)}\right)\right)\left[1-L_{k}(\beta)\right.\right. \\
& \left.+(1-m)+r L_{k}(\beta)\right]+(-\lambda E(I)) L^{\prime}{ }_{k}\left(E\left(L_{\left(e_{1}\right)}\right)+E\left(L_{\left(e_{2}\right)}\right)\right) \\
& -(\lambda E(1))\left(E ( L _ { ( e _ { 1 } ) } + E ( L _ { ( e _ { 2 } ) } ) ) \left[1-L_{k}(\beta)+E(H) \lambda E(I)\left(1-L_{k}(\beta)\right)\right.\right. \\
& \left.-\lambda E(I) L^{\prime}{ }_{k}+r \lambda E(I) L_{k}{ }_{k}\right] \\
& -\left[(\lambda E(I)) E(H)\left(1-L_{k}(\beta)\right)+\beta L^{\prime}{ }_{k}+E(H) \lambda E(I)\left(1-L_{k}(\beta)\right)\right. \\
+ & E\left(H^{2}\right)(-\lambda E(I))^{2}\left(1-L^{\prime}{ }_{k}(\beta)\right)+\beta E(I) E(H) L^{\prime}{ }_{k}+\beta(-\lambda E(I)) L^{\prime}{ }_{k} \\
& \left.\left.-\beta(-\lambda E(I))^{2} L^{\prime}{ }_{k} E(H)+\beta E\left(L_{k}{ }_{k}\right)(\lambda E(I))^{2}+r E\left(L_{k}{ }^{2}\right)(-\lambda E(1))^{2}\right]\right\}
\end{aligned}
$$

Substituting (33) in (32) we obtain $L_{q}$ in closed form.

Further, all the other queue performance measures can be found using Little's law $W_{q}=\frac{L_{q}}{\lambda^{+}}, \mathrm{W}=\frac{L}{\lambda^{+}}, \mathrm{L}=L_{q}+\rho$

\section{NUMERICAL ILLUSTRATION}

$$
\begin{gathered}
\lambda=4, \beta=2, r=06, m=05, \mu_{\left(e_{1}\right)}=25, \mu_{\left(e_{2}\right)}=35, \mu_{k}=28, \mu_{m}=3, \\
E\left(L_{\left(e_{1}\right)}\right)=\frac{1}{\mu_{\left(e_{1}\right)}}, E\left(L_{\left(e_{2}\right)}\right)=\frac{1}{\mu_{\left(e_{2}\right)}}, L_{k}^{\prime}=\frac{1}{\mu_{k}}, E(H)=\frac{1}{\mu_{m}}, \quad L_{k}(\beta)=\frac{\mu_{k}}{\mu_{k}+\beta}, E\left(L_{k}^{2}\right)=\frac{2}{\mu_{k}^{2}}, \\
E\left(H^{2}\right)=\frac{2}{\mu_{m}^{2}}, L_{k}^{\prime}(\beta)=\frac{\mu_{k}}{\left(\beta+\mu_{k}\right)^{2}}
\end{gathered}
$$

Table 1. Effect of change of ( $\beta=2,25,3,35,4)$.

\begin{tabular}{|c|c|c|c|c|c|}
\hline $\boldsymbol{Q}$ & $\boldsymbol{P}$ & $\boldsymbol{L} \boldsymbol{q}$ & $\boldsymbol{L}$ & $\boldsymbol{W} \boldsymbol{W}$ & $\boldsymbol{W}$ \\
\hline 0.6485 & 0.3515 & 8.5449 & 8.8964 & 2.1362 & 2.2241 \\
\hline 0.6917 & 0.3083 & 12.552 & 12.8602 & 3.138 & 3.2151 \\
\hline 0.7382 & 0.2618 & 16.556 & 16.8173 & 4.1389 & 4.2043 \\
\hline 0.7787 & 0.2213 & 20.983 & 21.2043 & 5.2457 & 5.3011 \\
\hline 0.8190 & 0.1810 & 25.237 & 25.4176 & 6.3092 & 6.3544 \\
\hline
\end{tabular}

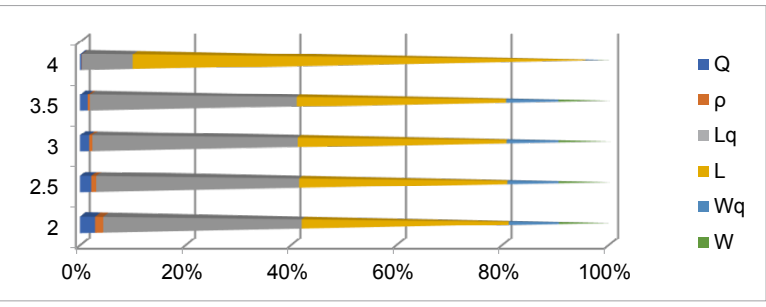

Graphic 3. Effect of change of $\beta$. 
From Table 1 and Figure 3, it is clear that if the probability in service interruption increases it leads to an increase in all the performance measures. Since the service interruption gets increased the idle time gets amplified and utilization factor is decreased.

Table 2. Effect of change of .

\begin{tabular}{|c|c|c|c|c|c|}
\hline $\boldsymbol{Q}$ & $\boldsymbol{P}$ & $\boldsymbol{L q}$ & $\boldsymbol{L}$ & $\boldsymbol{W q}$ & $\boldsymbol{W}$ \\
\hline 0.6485 & 0.3515 & 8.5449 & 8.8964 & 2.1362 & 2.2241 \\
\hline 0.645 & 0.355 & 8.6013 & 8.9563 & 2.1503 & 2.2399 \\
\hline 0.6391 & 0.3609 & 8.6967 & 9.0576 & 2.1742 & 2.2644 \\
\hline 0.6277 & 0.3723 & 8.9197 & 9.2077 & 2.2299 & 2.3019 \\
\hline 0.5977 & 0.4023 & 9.7597 & 10.162 & 2.4399 & 2.5405 \\
\hline
\end{tabular}

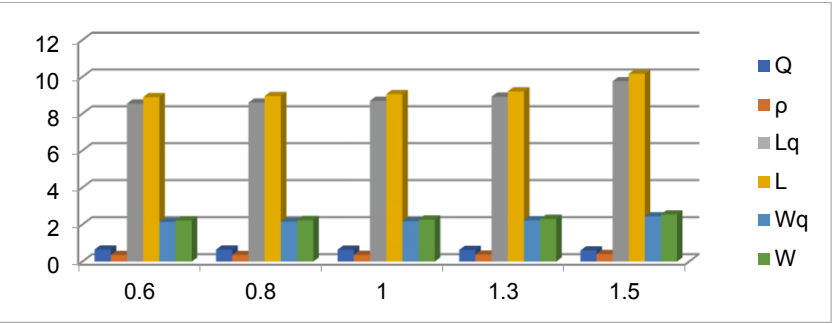

Graphic 4. Effect of change of $r$.

Table 2 indicates that, as the probability of repair rate gets increased, length of the queue is increased. Since the repair rate increased utilization factor gets increased and idle time gets decreased.

\section{CONCLUSIONS}

In this paper we have studied a batch arrival, two phases of essential administration and optional administration, service interruption, feedback service. This paper clearly analyses the steady state results and some queuing performance measures. Further this model can be extended by adding the concept of delay time, reneging, long vacation, short vacation etc. 


\section{REFERENCES}

Blumenthal, D. J., Bowers, J. E., Rau, L., Chou, H. F., Rangarajan, S., Wang, W., \& Poulsen, K. N. (2003). Optical signal processing for optical packet switching networks. IEEE Communications magazine, 41(2), S23-S29. https://doi.org/10.1109/ MCOM.2003.1179494

Boehm, R., Ghing, Y. G., Griffith, G., \& Saal, F. (1986). Standardized Fiber Optic transmission systems-A Synchronous optical network view. IEEE Journal on Selected Areas in Communications, 4(9), 1424-1431. https://doi.org/10.1109/JSAC.1986.1146482

Ghao, H. J., Shtirmer, G., \& Smoot, L. S. (1989). H-Bus: an experimental ATM-based optical premises network. Fournal of Light wave Technology, 7(11), 1859-1867. https:// doi.org/10.1109/50.45908

Gosares, S., Deutsch, D. N., Saniee, I., \& Wasem, O. J. (1995). SONET Toolkit: A Decision support system for Designing Robust and Cost-Effective Fiber-Optic Networks. Informs fournal on applied analytics, 25(1), 1-142. https://doi.org/10.1287/ inte.25.1.20

Hac, A., \& Mutlu, H. B. (1989). Synchronous optical network and broadband ISDN protocols. IEEE Computer, 22(11), 26-34. http://doi.org/10.1109/2.43524

Jue,J. P ., Yang, W. H., Kim, Y. G., \& Zhang, Q. (2009). Optical packet and burst switched networks: a review. IET communications, 3(3), 334-352. http://doi.org/10.1049/ietcom:20070606

Kang, G. S., Park, B. S., Shin, J. D., \& Jeong, J. M. (1995). A broadband ring network: Multichannel optical slotted ring. Computer Networks and ISDN Systems, 27(9), 13871398. https://doi.org/10.1016/0169-7552(94)00055-X

Lee, Y., Sherali, H. D., Han, J., \& Kim, S. (2000). A branch-and-cut algorithm for solving an intraring synchronous optical network design problem. Networks an International fournal, 35(3), 223-232. https://doi.org/10.1002/(SICI)10970037(200005)35:33.0.CO;2-J 
Maragathasundari, S., \& Balamurugan, B. (2015). A study on the performance analysis of a batch arrival queue with two stages of service Bernoulli schedule vacation extended vacation and service interruption. International journal of computer applications, 124(12), 33-37. https://www.researchgate.net/publication/295513726_A_Study_ on_the_Performance_Analysis_of_a_Batch_Arrival_Queue_with_Two_Stages_ of_Service_Bernoulli_Schedule_Vacation_Extended_Vacation_and_Service_ Interruption

Maragathasundari, S., \& Dhanalakshmi, K. S. (2018). Mobile adhoc networks problem-A queuing approach. International journal of communication networks and distributed system, 21(4). https://www.researchgate.net/profile/Dhanalakshmi_ Sadhasivan/publication/325987340_Mobile_Adhoc_Networks_MANETs_ Problem-_A_Queueing_approach/links/5c6270c792851c48a9cd5656/MobileAdhoc-Networks-MANETs-Problem-A-Queueing-approach.pdf

Maragathasundari, S., \& Sowmiah, S. (2016). A study on the analysis of performance measure of bulk input queue with $\mathrm{N}$ type of additional optional service, service interruption and deterministic vacation. Imperial fournal of Interdisciplinary Research, 2(8), 1436-1444.

Maragathasundari, S., \& Srinivasan, S. (2012). Analysis of M/G/1 feedback queue with three stages and multiple server vacation. Applied mathematical sciences, 6(125), 6221-6240. http://m-hikari.com/ams/ams-2012/ams-125-128-2012/ maragathaAMS 125-128-2012.pdf

Maragathasundari, S., \& Srinivasan, S. (2015). A Non-Markovian Multistage Batch arrival queue with break down and reneging. Mathematical problems in engineering, Article ID 519579. http://dx.doi.org/10.1155/2014/519579

Way, W. I., Smith, D. A., Johnson, J. J., \& Izadpanah, H. (1992). A self-routing WDM high-capacity SONET ring network. IEEE Photonics Technology Letters, 4(4), 402-405. https://doi.org/10.1109/68.127228 


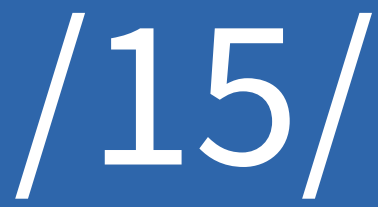




\section{SURVEY ON VARIOUS PERSPECTIVES OF RAMAN AMPLIFIERS}

Sumathy Raju

Assistant Professor, ECE, Kalasalingam Academy of Research and Education,

Virudhunagar, Tamil Nadu, (India).

E-mail: sumathyraju1110@gmail.com ORCID: https://orcid.org/0000-0001-9871-1780

Muthukumar Arunachalam

Associate Professor, ECE, Kalasalingam Academy of Research and Education,

Virudhunagar, Tamil Nadu, (India). E-mail: muthuece.eng@gmail.com ORCID: https://orcid.org/0000-0001-8070-3475

\section{Citación sugerida:}

Raju, S., y Arunachalam, M. (2020). Survey on various perspectives of raman amplifiers. 3C Tecnología. Glosas de innovación aplicadas a la pyme. Edición Especial, Marzo 2020, 247-259. http://doi. org/10.17993/3ctecno.2020.specialissue4.247-259

\section{Suggested citation:}

Raju, S., \& Arunachalam, M. (2020). Survey on various perspectives of raman amplifiers. 3C Tecnología. Glosas de innovación aplicadas a la pyme. Edición Especial, Marzo 2020, 247-259. http://doi. org/10.17993/3ctecno.2020.specialissue4.247-259 


\section{ABSTRACT}

Raman Amplifier (RA) is the fiber amplifier that follows Stimulated Raman Scattering (SRS) mechanism. For broadband amplification it is used, because of low noise and better gain. Raman amplification was investigated in multiple views. Many research works had focused in the views of pumping schemes, gain flattening, transmission system and noise analysis. In this paper, Raman amplification is studied in the views of varying Refractive Index profile of core, varying core gap radius, hybrid combination of RA with Erbium Doped Fiber Amplifier (EDFA). This paper also studies the investigations of this hybrid combination in Dispersion compensation at $\mathrm{C}$ and $\mathrm{S}$ bands, recycling of pump power and location of EDFA.

\section{KEYWORDS}

Refractive index profile, Hybrid Amplifiers, Dispersion compensation. 


\section{INTRODUCTION}

In long distance Optical communication system, whenever the signal strength becomes low, we need to boost up it. At the beginning, Regenerators were used to rejuvenate the signal. Here, the optical signal is converted into the electrical signal. After the regeneration, the electrical signal is again converted into optical signal which is a very costly process. Then Semiconductor Optical Amplifiers (SOA) were used. From the studies (Elndash, Mohammed \& Rashed 2010; Fugihara \& Pinto, 2008), these amplifiers have disadvantages like, low output power, high noise figure. In order to avoid these, Fiber amplifiers were used. Fiber amplifiers are the amplifiers where a fiber itself becomes amplification medium by using pumping. There are two fiber amplifiers are mostly used. One is EDFA and second one is Raman Amplifier. In EDFA, a rare earth element Erbium is doped. When EDFA is compared with RA, gain bandwidth is low and wavelength of operation is limited. But in RA, at any wavelength we can obtain amplification. So Raman amplifications are now mostly used in long distance optical communication system. The researches (Bromage, 2004; Islam, 2002; Namiki \& Emori, 2001) concluded that Raman amplification has important advantages of having very low noise and suitable for broadband applications, particularly in WDM systems, where simultaneous amplification is required for multi-channel light. Raman amplification may be Distributed Raman amplification (DRA) or lumped Raman amplification. Dhir and Gupta (2013), found that DRA has benefits like high gain, high data rate and less fiber loss. Raman amplifier also provides better performance when it is combined with EDFA or SOA. Many research works had been done in various aspects like flat gain amplification, noise performance, pumping schemes, \& hybrid system. Lot of ideas to pump powers and wavelengths selection was discussed in the literatures (Ferreira, Cani, Pontes \& Segatto 2011; Neto, Teixeira, Wada, \& André, 2007). Raman amplification is happening from the process SRS. SRS is a notable non-linear effect which affects the Signal to Noise Ratio (SNR) in a WDM system. It can also be used for amplification of the optical signals in a long distance optical communication link. The spontaneous Raman scattering was found by Sir C. V. Raman. In case of this scattering, a small quantity of the incident light is changed into light signal of either low or high frequency. SRS gives the amplification if the pump signal with suitable wavelength enters the fiber (Dhir et al., 2013; Ferreira et al., 2011; Fugihara et al., 2008). In SRS, photon form pumping source is 
absorbed by material and emits a photon with energy at its vibrational state. In fact, energy is transmitted from a high frequency optical signal to lower frequency optical signal (Dhir and Gupta, 2014). Raman amplification is not only possible for single mode fiber but also for multimode fiber which was discussed in a research (Polley \& Ralph, 2007). In this work, For fundamental mode LP0,1, the Raman gain was compared. This multimode amplification is mainly used in space-division multiplexing (Antonelli, Mecozzi \& Shtaif 2013; Namiki \& Emori, 2001). We can make any fiber into a Raman amplifier by suitable selection of pumping wavelength (Anwar \& Aly, 2010). This paper investigates two important views in RA. Section 2 investigates about RA performance in various Refractive Index Profiles and Section 3 investigates about the performance of RA with EDFA hybrid configuration.

\section{INVESTIGATIONS OF REFRACTIVE INDEX PROFILE (RIP)}

Raman amplifiers are used not only for amplification but also for dispersion compensation. In Dispersion compensation, the refractive index profile of fiber plays a major role.

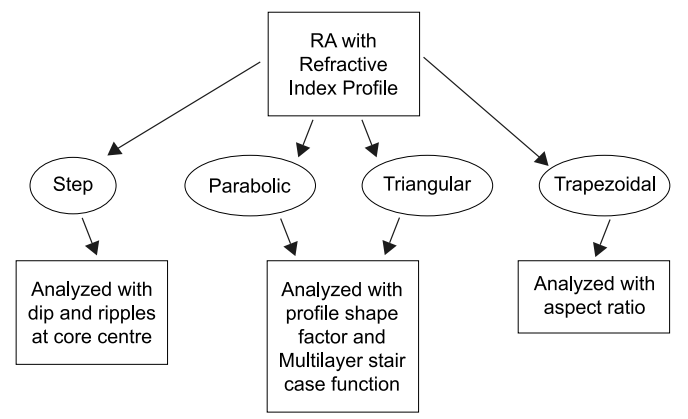

Figure 1. Study of Raman Amplifier based on Refractive Index Profile.

In the design of RA, the operating wavelength range is adjusted by correctly selecting suitable RIP structure and inner core radius. In this paper, RIP based performance of RA is studied as per Figure 1 Among step, parabolic and triangular refractive index profiles, a parabolic profile provides good effective Raman gain results at 20Gbps. The paper (Chan \& Premaratne 2007) also reports the change in dispersion co-efficient and gain as the function of RIP. For obtaining better flattening of Raman gain, the core gap radius of a fiber is varied with step index profile. Additional to this, large negative dispersion coefficient is achieved by varying core gap radius (Bandyopadhyay \& Sarkar 2013) From this, dispersion compensation is achieved at C and S bands. A research (Pramanik, Das, \& Sarkar, 2010) 
found that Trapezoidal index profile also affects the gain performance of RA. The aspect ratio $\mathrm{S}$ is modified, and same phase matching is achieved for various values of core radius. During the design of RA, step index profile at both inner and outer cores was considered in another research (Pramanik \& Sarkar, 2014). This work says that the occurrence of axial dip at core center is unavoidable. Up to $0.25 \%$ dip depth and $25 \%$ dip width, RA is performing as perfect as RA without any dip or profile imperfections. But above this limit of dip depth and dip width, the performance becomes poor. This paper concluded that step index profile in core is most excellent for better performance of RA.

From the investigations of these research works, the Raman gain of various profiles are observed at $1550 \mathrm{~nm}$ wavelength window for fundamental mode $\operatorname{LP}(0,1)$ and tabulated in Table 1.

Table 1. Raman Gain values at 1550nm Window.

\begin{tabular}{|c|c|}
\hline Type of profile & Raman gain $(\mathbf{m} / \mathbf{W})$ \\
\hline Parabolic index & $0.7 \times 10-13$ \\
\hline Triangular index & $0.62 \times 10-13$ \\
\hline Step index & $0.76 \times 10-13$ \\
\hline Trapezoidal index & $1.04 \times 10-13$ \\
\hline
\end{tabular}

From this Table 1, trapezoidal index profile gives the better Raman gain. But even with dip at core centre step index profile gives the better performance (Pramanik et al., 2014).

\section{INVESTIGATIONS ON HYBRID COMBINATION OF EDFA \& RAMAN AMPLIFIERS}

Many research works have used benefits from both the fiber amplifiers RA and EDFA. When RA is combined with EDFA, cross talk becomes very less even for channel spacing of $0.4 \mathrm{~nm}$ and $0.2 \mathrm{~nm}$. This hybrid RA and EDFA performance is inspected for 16X10Gbps DWDM system (Singh \& Kaler, 2015). And this paper concluded that hybrid RA and EDFA is better than other hybrid optical amplifiers. Better power utilization also possible with this hybrid RA and EDFA. It is investigated in the research (Lee, Chang, Han, Kim, Chung \& Lee, 2004) that recycling the residual Raman pump to make pumping on EDFA. Choosing proper pump wavelength is the only thing to be considered. This gives the possibility for the design of broadband amplifiers with high gain. 


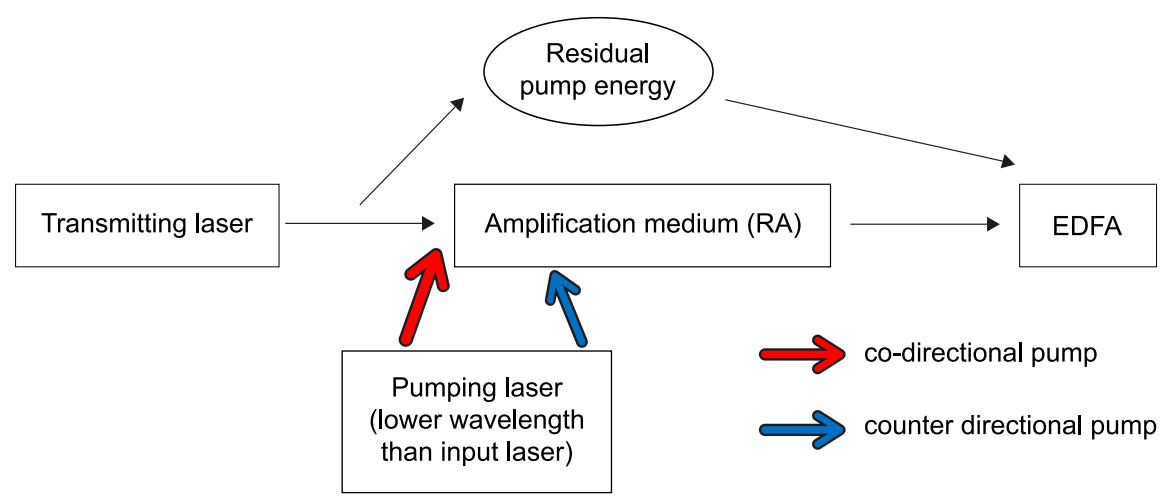

Figure 2. RA-EDFA hybrid configuration with single pump.

As shown in Figure 2, the pumping may be given either in forward direction (co-direction) or in backward direction (counter-direction).

In hybrid optical amplifiers, the site of the Erbium doped fiber severely affects the performance of the dispersion compensation of Raman/EDF and this is discussed in a research (Ali, Abdullah, Jamaludin, Al-Mansoori, Al-Mashhadani \& Abass, 2013). EDF may be placed before RA or after RA. In this work, these are considered as two cases. A 10m EDF and $7 \mathrm{Km}$ Dispersion Compensating Fiber (DCF) is considered. Here, DGF is acting as Raman Amplification medium. In first case, EDF is placed before RA. And in second case EDF is placed after RA. In this research, fiber has the following specifications. They are $0.55 \mathrm{~dB} / \mathrm{Km}$ of attenuation coefficient, $98 \mathrm{ps} / \mathrm{nm} . \mathrm{km}$ of dispersion co efficient, and 15.3 $\mu \mathrm{m} 2$ of effective area. And EDF has the following specifications. Erbium concentration of $440 \mathrm{ppm}, 2.2 \mu \mathrm{m}$ core radius, and effective area of $15.2 \mu \mathrm{m}^{2}$. If we place the RA firstly and EDFA secondly, then we obtain large input signal gain, noise figure and gain variation. RAMAN-SOA and RAMAN -EDFA are analyzed in the research of Upma (2015). This research proposed the 8 channel transmitter with constant attenuation value $0.2 \mathrm{db} / \mathrm{Km}$. And data speed of $10 \mathrm{Gbps}$ is considered. Under such case, Raman-EDFA gives highest $\mathrm{Q}$ factor of 19.92db, RAMAN-SOA provides highest eye opening, Raman -EDFA provides smallest jitter 0.0243. so RAMAN -EDFA is a hopeful alternative to all other hybrid amplifiers. With the help of mono pump source, dispersion compensating Raman/EDFA hybrid amplifier is achieved. Also overall power conversion efficiency is increased. Here mono pump source having two lasers is used for Dispersion Compensating Fiber (DCF) which has Raman Amplification. The remaining power from this DCF is again recycled 
and utilized by EDFA. The laser pumps are operating at $1455 \mathrm{~nm}$ and $1465 \mathrm{~nm}$. A total pump power of $500 \mathrm{~mW}$ is launched into a $12.6 \mathrm{Km}$ DCF. Effective power utilization and larger amplifier efficiency is obtained in the research (Lee, Chang, Han, Chung, Kim \& Lee, 2005).

Like Erbium, Ytterbium can also be used as fiber amplifier. By Yb-Raman combined non linear amplifier, an improved power of $1.5 \mathrm{~kW}$ was obtained in the wavelength of $1100 \mathrm{~nm}$ to $1200 \mathrm{~nm}$. In this work (Zhan, Tao, Zhou, Wang, \& Xu, 2014), the amplifier is seeded by 1070 and 1120nm signal lasers simultaneously.

For 16 channel system (Lee, Oh, Lee, Lee, \& Hwang, 2004), Q factor \& BER for RamanEDFA \& EDFA-RAMAN-EDFA are same for short distance. For longer distance, EDFARAMAN-EDFA has largest $Q$ value among all. For 32 channel system, RAMAN-EDFA provides good output power; BER \& Q factor. In the hybrid combination of RamanEDFA, EDFA may be used in parallel configuration and residual pumping is done. In order to minimize the cost, mono pump wavelength is used in Raman amplifier. Raman amplifier in the role of DCF has two benefits. First one is, obtaining low loss and dispersion compensation at the same time. Second is, amplification is done at wider band in optical wavelength window by changing the pump wavelength and it was discussed in a research (Singh \& Kaler, 2015). In order to maximize capacity for amplification scheme and transmission distance, a variety of combinations of three 16QAM based coded modulation schemes with spectral efficiencies 4.86/5.4 for C+L EDFA experiment and 5.45/6.14 [bits/ $\mathrm{Hz}]$ are used in this paper (Cai et al., 2015).

A different research is done in this work (Mahran, 2015). Here, the bending loss in EDFA makes the gain of hybrid amplifier to increase up to 7db more than normal EDFA/Raman. OSNR calculations also show a better performance. In this paper bending radius is chosen as $4 \mathrm{~mm}$, EDFA is taken with length $10 \mathrm{~m}$ where, forward pump power in the range of 100 $500 \mathrm{~mW}$, Raman amplifier length is chosen between 12 to55Km, where backward pump power in the range of $80-200 \mathrm{mw}$ and input signal power is $-20 \mathrm{dBm}$. Even for reduced channel spacing, this hybrid RA-EDFA provides better performance. This research (Singh \& Kaler, 2012) investigated various combination of hybrid amplifiers. 100 channels were 
used at channel spacing of $6.25 \mathrm{GHz}$. Quality factors, acceptable bit rate, Bit Error Rate (BER) were calculated for all the configurations.

Pump power of RA is unused in Dispersion compensating modules (DCM). This wastage of pump power is avoided and utilized by using FBG at one side of DCM. This DCM is designed for short or medium distance mostly from $50 \mathrm{Km}$ to $100 \mathrm{Km}$ (Nicholson, 2003). Both the benefits of dispersion compensation and effective power utilization were obtained from this research.

\section{SUMMARY}

This paper assesses about various perspectives of Raman Amplifiers. The effort towards mitigating dispersion, improving gain bandwidth, effective power utilization, obtained low noise performance, dependency of Refractive index profile using RA and corresponding researches were described in this paper. When hybrid configuration of RA-EDFA is considered, the innovative research papers of bending loss in EDFA for dispersion compensation and locating EDFA for low noise were also depicted in this paper. 


\section{REFERENCES}

Ali, M. H., Abdullah, F., Jamaludin, M. Z., Al-Mansoori, M. H., Al-Mashhadani, T. F., \& Abass, A. K. (2013). Effect of EDF position on the performance of hybrid dispersion-compensating Raman/EDF amplifier. In IEEE 4th International Conference on Photonics (ICP) (pp. 187-189). IEEE. https://doi.org/10.1109/ICP.2013.6687109

Antonelli, G., Mecozzi, A., \& Shtaif, M. (2013). Raman amplification in multimode fibers with random mode coupling. Optics letters, 38(8), 1188-1190. https://doi. org/10.1364/OL.38.001188

Anwar, N. M., \& Aly, M. H. (2010). Backward Pumped Distributed Fiber Raman Amplifiers. 27th National Radio Science Conference. https://www.researchgate.net/ publication/259181526_Backward_Pumped_Distributed_Fiber_Raman_ Amplifiers

Bandyopadhyay, P. K., \& Sarkar, S. (2013). Effect of variation of core gap radius on the performance of dual concentric core Raman fiber amplifier. Optics Communications, 300, 27-32. https://doi.org/10.1016/j.optcom.2013.02.029

Bromage, J. (2004). Raman amplification for fiber communications systems. Fournal of Lightwave Technology, 22(1), 79-93. https://doi.org/10.1109/JLT.2003.822828

Cai, J. X., Sun, Y., Zhang, H., Batshon, H. G., Mazurczyk, M. V., Sinkin, O. V., ... \& Pilipetskii, A. (2015). $49.3 \mathrm{~Tb} / \mathrm{s}$ transmission over $9100 \mathrm{~km}$ using C+ L EDFA and $54 \mathrm{~Tb} / \mathrm{s}$ transmission over $9150 \mathrm{~km}$ using hybrid-Raman EDFA. Journal of Lightwave Technology, 33(13), 2724-2734. https://doi.org/10.1109/JLT.2015.2409846

Chan, A. C. O., \& Premaratne, M. (2007). Dispersion-compensating fiber Raman amplifiers with step, parabolic, and triangular refractive index profiles. Fournal of lightwave technology, 25(5), 1190-1197. https://doi.org/10.1109/JLT.2007.893033

Dhir, E. J., \& Gupta, E. V. (2013). Improvement of Gain with Figure of Merit in Discrete Raman Amplifier. International Fournal of Computer Science and Communication Engineering, (IFCSCE), 22-24. 
Dhir, J., \& Gupta, V. (2014). Improvement of Raman Gain with Different Parameters in Discrete Raman Amplifier. International Journal of Engineering Research and Applications (IJERA). National Conference on Advances in Engineering and Technology (pp. 16-19). http:// www.ijera.com/special_issue/AET_Mar_2014/ECE/Version\%20\%201/D1619. pdf

Elndash, A., Mohammed, N. A., \& Rashed, A. N. Z. (2010). Comparison performance evolution of different transmission techniques with bi-directional distributed Raman gain amplification technique in high capacity optical networks. International Journal of Physical Sciences, 5(5), 484-495. https://www.semanticscholar.org/paper/ Comparison-performance-evolution-of-different-with-Elndash-Mohammed/7c085 d6ce3d37b342167a0bb8e624eceea036646

Ferreira, G. G., Gani, S. P. N., Pontes, M.J., \& Segatto, M. E. V. (2011). Optimization of distributed Raman amplifiers using a hybrid genetic algorithm with geometric compensation technique. IEEE Photonics Fournal, 3(3), 390-399. https://doi. org/10.1109/JPHOT.2011.2140366

Fugihara, M. G., \& Pinto, A. N. (2008). Low-cost Raman amplifier for CWDM systems. Microwave and Optical Technology Letters, 50(2), 297-301. https://doi.org/10.1002/ mop. 23075

Islam, M. N. (2002). Raman amplifiers for telecommunications. IEEE fournal of selected topics in Quantum Electronics, 8(3), 548-559. https://doi.org/10.1109/JSTQE.2002.1016358

Lee, H. H., Oh, J. M., Lee, D., Lee, G. W., \& Hwang, S. T. (2004). Demonstration of $16 \times 10 \mathrm{~Gb} / \mathrm{s}$ WDM transmission over $4 \times 40 \mathrm{~km}$ of SMF using linear optical amplifiers combined with Raman-pumped dispersion compensation fibers under dynamic add/drop situations. In Optical Fiber Communication Conference, Technical Digest (CD) (Optical Society of America, 2004), paper WG2. https://www.osapublishing.org/ abstract.cfm? uri $=\mathrm{OFG}-2004-\mathrm{WG} 2$ 
Lee, J. H., Chang, Y. M., Han, Y. G., Ghung, H., Kim, S. H., \& Lee, S. B. (2005). A detailed experimental study on single-pump Raman/EDFA hybrid amplifiers: static, dynamic, and system performance comparison. Fournal of light wave technology, 23(11), 3484. https://doi.org/10.1109/JLT.2005.857773

Lee, J. H., Chang, Y. M., Han, Y. G., Kim, S. H., Ghung, H., \& Lee, S. B. (2004). Dispersion-compensating Raman/EDFA hybrid amplifier recycling residual Raman pump for efficiency enhancement. IEEE Photonics Technology Letters, 17(1), 43-45. https://doi.org/10.1109/LPT.2004.837264

Mahran, O. (2015). Performance study of macro-bending EDFA/Raman hybrid optical fiber amplifiers. Optics Communications, 353, 158-164. https://doi.org/10.1016/j. optcom.2015.05.030

Namiki, S., \& Emori, Y. (2001). Ultrabroad-band Raman amplifiers pumped and gainequalized by wavelength-division-multiplexed high-power laser diodes. IEEE fournal of Selected Topics in Quantum Electronics, 7(1), 3-16. https://doi.org/10.1109/2944.924003

Neto, B., Teixeira, A. J., Wada, N., \& André, P. S. (2007). Efficient use of hybrid genetic algorithms in the gain optimization of distributed Raman amplifiers. Optics express, 15(26), 17520-17528. https://doi.org/10.1364/OE.15.017520

Nicholson, J. W. (2003). Dispersion compensating Raman amplifiers with pump reflectors for increased efficiency. Fournal of lightwave technology, 21(8), 1758. https://doi. org/10.1109/JLT.2003.815495

Polley, A., \& Ralph, S. E. (2007). Raman amplification in multimode fiber. IEEE Photonics Technology Letters, 19(4), 218-220. https://doi.org/10.1109/LPT.2006.890752

Pramanik, S., \& Sarkar, S. (2014). Effect of dip in refractive index profile on fiber Raman gain amplifier performance. Optics Communications, 329, 145-150. https:// doi.org/10.1016/j.optcom.2014.05.016

Pramanik, S., Das, G., \& Sarkar, S. N. (2010). Comparative study of the influence of the aspect ratio of trapezoidal index profiles on the performance of a fiber Raman amplifier. Optical Engineering, 49(5), 055001. https://doi.org/10.1117/1.3421554 
Singh, S., \& Kaler, R. S. (2012). Investigation of hybrid optical amplifiers for dense wavelength division multiplexed system with reduced spacings at higher bit rates. Fiber and Integrated Optics, 31(3), 208-220. https://doi.org/10.1080/01468030.2012 .666818

Singh, S., \& Kaler, R. S. (2015). Performance optimization of EDFA-Raman hybrid optical amplifier using genetic algorithm. Optics \& Laser Technology, 68, 89-95. doi: https://doi.org/10.1016/j.optlastec.2014.10.011

Singh, S., \& Kaler, R. S. (2015). Review on recent developments in hybrid optical amplifier for dense wavelength division multiplexed system. Optical Engineering, 54(10), 100901. https://doi.org/10.1117/1.OE.54.10.100901

Upma, P. S. (2015). Performance Comparison Of Optical Amplifiers And their Hybrid Configurations In 8x10 Gbps WDM Based Optical Network. International fournal of Engineering Research and General Science, 3(3), 154-158. http://pnrsolution.org/ Datacenter/Vol3/Issue3/233.pdf

Zaki Rashed, A. N. (2011). New trends of forward fiber Raman amplification for Dense Wavelength Division Multiplexing (DWDM) photonic communication networks. International Fournal on Technical and Physical Problems of Engineering, 3, 30-39. http:// www.iotpe.com/IJTPE/IJTPE-2011/IJTPE-Issue7-Vol3-No2-Jun2011/6-IJTPEIssue7-Vol3-No2-Jun2011-pp30-39.pdf

Zhang, H., Tao, R., Zhou, P., Wang, X., \& Xu, X. (2014). 1.5-kW Yb-Raman combined nonlinear fiber amplifier at $1120 \mathrm{~nm}$. IEEE Photonics Technology Letters, 27(6), 628-630. https://doi.org/10.1109/LPT.2014.2386973 


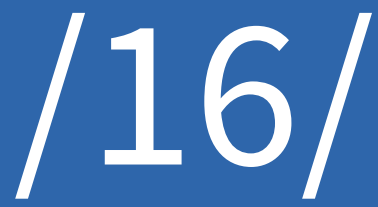




\section{MUSIC RECOMMENDATION SYSTEM BASED ON FACIAL EMOTION RECOGNITION}

Deny John Samuvel

Department of Electronics and Communication Engineering, Kalasalingam Academy of Research and Education,

Krishnankoil, Virudhunagar Dt., (India). E-mail: deny.j@klu.ac.in ORCID: http://orcid.org/0000-0001-6515-3575

B. Perumal

Department of Electronics and Communication Engineering,

Kalasalingam Academy of Research and Education, Krishnankoil, Virudhunagar Dt., (India). E-mail: palanimet@gmail.com ORCID: https://orcid.org/0000-0003-4408-9396

Muthukumaran Elangovan

Department of Electronics and Communication Engineering,

Dr. B. R. Ambedkar Institute of Technology, Pahargaon, Port Blair, (India). E-mail: reachmkumaran@gmail.com ORCID: https://orcid.org/0000-0002-0763-9902

\section{Citación sugerida:}

Samuvel, D. J., Perumal, B., y Elangovan, M. (2020). Music recommendation system based on facial emotion recognition. 3C Tecnología. Glosas de innovación aplicadas a la pyme. Edición Especial, Marzo 2020, 261-271. http://doi.org/10.17993/3ctecno.2020.specialissue4.261-271

\section{Suggested citation:}

Samuvel, D. J., Perumal, B., \& Elangovan, M. (2020). Music recommendation system based on facial emotion recognition. 3C Tecnología. Glosas de innovación aplicadas a la pyme. Edición Especial, Marzo 2020 , 261-271. http://doi.org/10.17993/3ctecno.2020.specialissue4.261-271 


\section{ABSTRACT}

Face recognition technology has widely attractedattention due to its enormous application value and marketpotential. It is being implemented in various fields like securitysystem, digital video processing, and many such technologicaladvances. Additionally, music is the form of art, which isknown to have a greater connection with a person's emotion. Ithas got a unique ability to lift up one's mood. Relatively, thispaper focuses on building an efficient music recommendationsystem which determines the emotion of user using FacialRecognition techniques. The algorithm implemented wouldprove to be more proficient than the existing systems. Moreover, on a larger dimension, this would render salvage oftime and labor invested in performing the process manually. The overall concept of the system is to recognize facial emotionand recommend songs efficiently. The proposed system will beboth time and cost efficient.

\section{KEYWORDS}

Recognition, Artificial intelligence, OpenCV Application. 


\section{INTRODUCTION}

Artificial intelligence, an extensive, prominent andimperative domain that has attracted a lot of researchers andprograms in recent times. This particular domain has takenover the world in very short notice. It is incorporated in over daily life in the form of chatbots, digital assistants like Siriand several other technology-based systems. One of the most prominent powers up of artificialintelligence is face recognition techniques. The basicexample of its usage is the grouping of Google Photos of aparticular person.

There are many existing systems that could recognize facialemotions. On the other hand, there are systems thatrecommend music. Bringing together, a system which willrecommend music by recognizing the mood of the user fromfacial emotions is the overall concept described in the paper. Emotion recognition would have larger scope in the nearfuture in fields like robotics for efficient sentimentalanalysis without the involvement of another human.

\section{RELATED WORK}

A few methodologies have been proposed and embraced to group human feelings successfully. Most of themethodologies laid their emphasis on seven essential feelings which are steady over age culture or different characters.

Describes the advantages of using OpenCV, especially the Adaboost algorithm, in the process of face recognition. Detecting and recognition of face in complicated color images can be achieved using a combination of a particular algorithm with AdaBoost algorithm. It also talks about the disadvantages of using a timer in face detection.

Proposes on utilizing Support Vector Machines (SVM) as the primary characterization technique to order eight facial feelings. The faces distinguished utilizing channels in OpenCV and changed over to Greyscale. The paper likewise explains on robotized constant coding of outward appearances in nonstop video gushing, which is feasible forapplications in which frontal perspectives can be accepted utilizing webcam.

The creator proposed a calculation to produce a subset ofa unique playlist or a custom playlist related to the feeling perceived. The picture to be prepared was acquired from 
aweb camera or the hard circle itself. The picture is expose to improvements, where a few mapping and upgrade procedures are connected to reestablish required differentiation of the picture. Preparing and arrangement are maintained by "one versus all" approach of SVM to encourage multi-class characterization.

Proposes on the utilization of profound convolutional neural networks. It depends on solid face acknowledgment convolutional systems, which can be effectively tweaked toplay out the feeling acknowledgment task. Visual models are supplemented with sound highlights for better face acknowledgment.

Aids in the music suggestion framework which is additionally a significant module of the proposed framework. It discusses highlights to be removed from the music to characterize its mind-set.

The paper depicts utilizing Thayer's model of mind-sets toperceive the state of mind of the music piece. The edge level of a music piece is resolved and the feeling it brings is perceived via prepared neural systems.

\section{METHODOLGY}

Compared to other algorithms used in previous systems, the proposed algorithm is proficient enough to battle large pose variations. Large pose variations tend to disrupt the efficiency of pre-existing algorithms. To reduce this Standard image input format is taken. Few systems detect the faces first and then locate them. On the other hand, rarely, some other algorithms detect and locate the faces at the same time. Every face detection algorithm usually has common steps. First, to achieve a response time, then to perform data dimension. Focusing on data dimension a few algorithms extract facial measurements and the next react certain relevant facial region. Advantages of the proposed algorithm Using the static image gives a great advantage on the defect of pose variations. The three most faced problems are the presence of unidentified elements like glasses or beard, quality of static images and unidentifiable facial gesture. Face Feature Extraction Pictures are spoken to as weight edeigen vectors that are consolidated and known as "Eigenfaces". One of the focal points taken by Eigen faces is the comparability between the pixels among pictures by methods for their covariance network. 
Following are the means required to perceive the outwardappearances utilizing this Eigenfaces approach:

Let $X=\left\{x_{1}, x_{2}, \ldots, x_{n}\right\} x_{i} \in R^{d}$

Here $X$ be a random vector with observations.

1. Calculate the mean $\mu$ :

$$
\mu=\frac{n}{1} \sum_{i=1}^{n} x_{i}
$$

2. Calculate the covariance matrix $S$ :

$$
S=\frac{n}{1} \sum_{i=1}^{n}\left(x_{i}-\mu\right)\left(x_{i}-\mu\right)^{T}
$$

3. Compute the eigenvectors $v_{i}$ and eigenvalues $\lambda_{\mathrm{i}}$ of $S$ :

$$
S v_{i}=\lambda_{i} v_{i}, i=1,2, \ldots, n
$$

4. The eigenvectors are arranged by their egeinvalue in descending order:

$$
y=W^{T}(x-\mu)
$$

5. Calculate eigenfaces.

Eigen Faces: Not all the parts of the face are important for emotion recognition. This key fact is considered to be important anduseful. Face recognition techniques focus on recognizingeyes, nose, cheek and forehead and how the change with respect to each other. Overall, the areas with maximumchanges, mathematically, areas with high variations are targeted. When multiple faces are considered, they are compared by detecting these parts of the faces becausethese parts are the most useful and important parts of a face. They tend to catch the maximum change among faces, specifically, the change that helps to differentiate one face from the other. This is how Eigen Faces face recognizer works. 


\section{SYSTEM ARCHITECHURE}

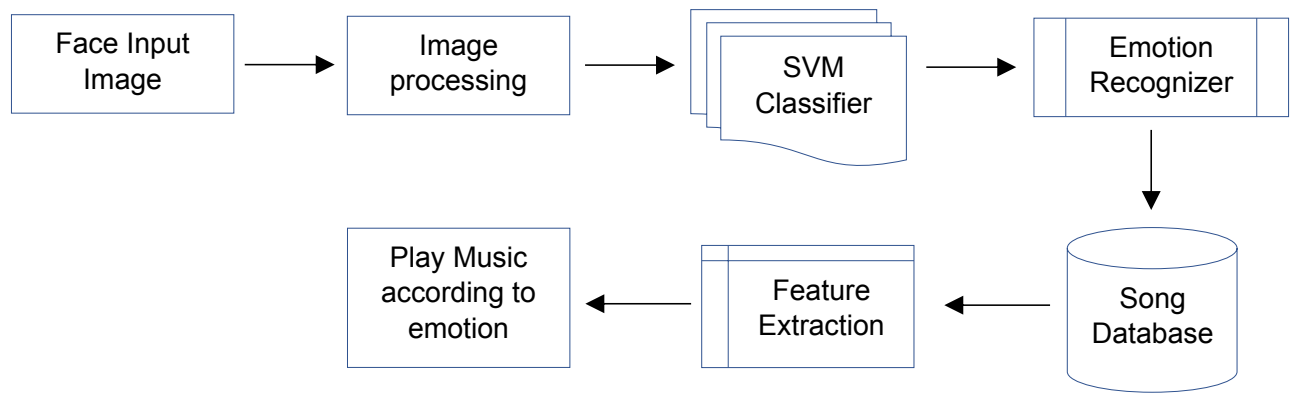

Graphic 1. Block Diagram.

The proposed framework is first prepared to distinguish a face from a static picture. When the information picture is perceived, the picture is handled. The picture isexposed to SVM classifiers for subtleties to perceive thefeeling displayed by the face. The subtleties recuperated from the image are utilized by the feeling classifier to discover feeling.

The song database and feature extraction module function simultaneously. The songs are disintegrated into several music pieces and the mood of the song is recognized. The songs are stored based on the mood detected. Once the emotion recognizer reports the mood, the songs pertaining to the mood are played by the music player.

\section{MODULE IDENTIFICATION}

Face Detection and Recognition: Facial expressions are powerful reflections of the emotional state of a person. In this section, we will discusshow images with human faces can be processed in order to detect the emotions presented in them. Various algorithms are used for face recognition. Here we are using the OpenCV to detect the face in the image. Eigenfaces algorithm is usedto recognize the face. The algorithms used for local feature extraction are Local Binary Patterns, Direct Cosines Transform, and Gabor Wavelets.

To depict progressively trademark highlights of thespecific chose face most noteworthy Eigenvalues of the Eigenvector will be picked as the ideal eigenface. Most noteworthy Eigenfaces with low Eigenvalues could be discarded since they coordinated just a little piece of trademark highlights of the countenances. 


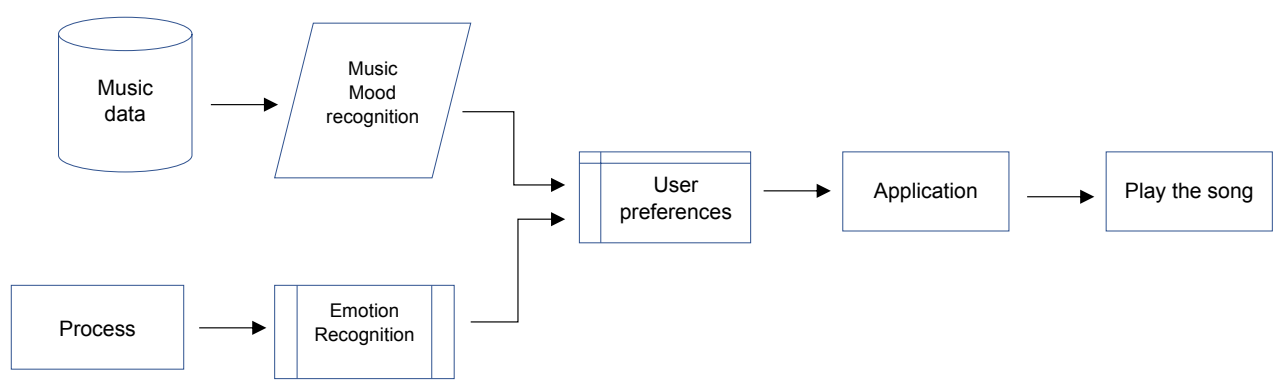

\section{Graphic 2. Module Explanation.}

Music Feature: Music can be recommended based on available information such as the album and artist. Another way of classifying the mood based on pitch and rhythm. Unfortunately, this will lead to predictable recommendations. For example, recommending songs basedon the artists that the user is known to enjoy is not particularly useful. With developing procedures, the utilization of Neural Networks has turned out to be progressively famous. We utilize an Artificial Neural Network (ANN) to arrange the melodies in individual classes. The dataset we utilized for preparing the model is Million Song Dataset given by Kaggle. The information comprises of two records: metadata document and triplet document. The metadat_file contains the title, song_id, artist_name, andrelease_by. Thetriplet_file contains user_id, song_id and listen time. 


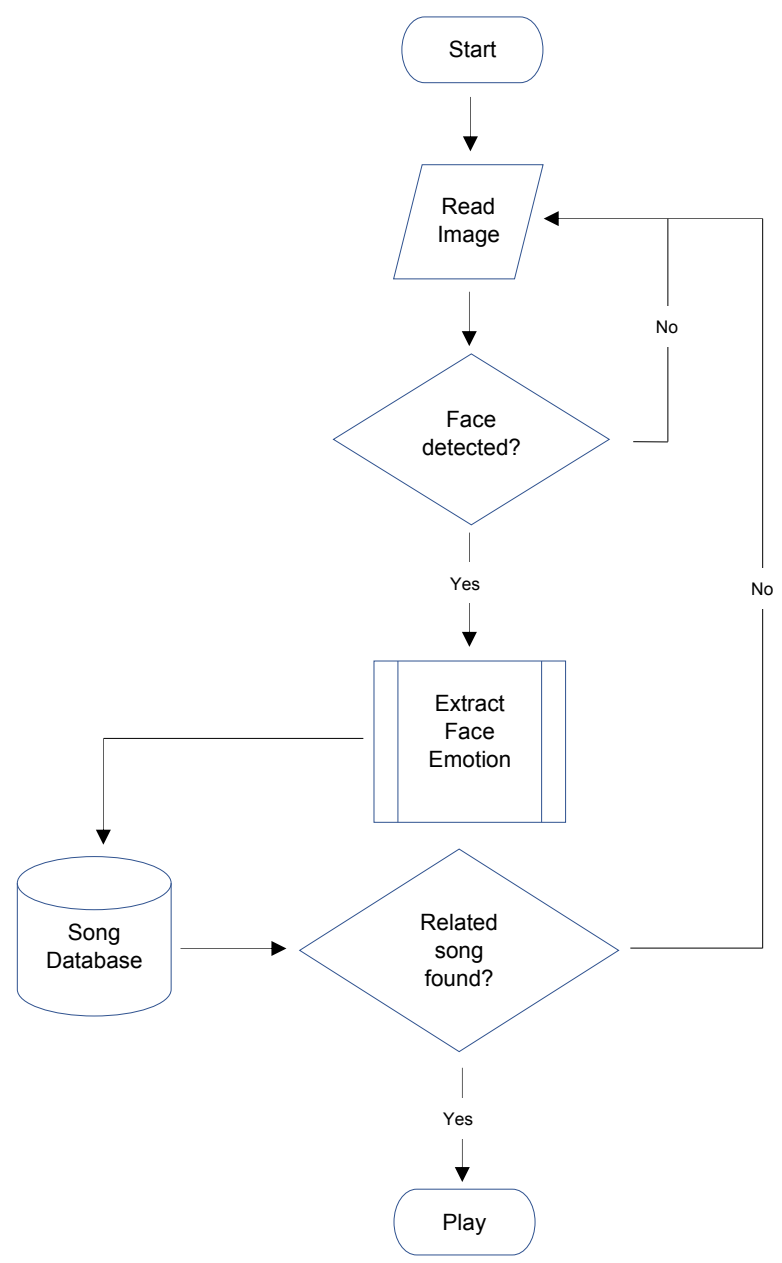

Graphic 3. Flow diagram of the proposed system.

\section{CONCLUSION AND FUTURE ENHANCEMENT}

A simple system is proposed here for the music recommendation using face emotion recognition. It suggests music by extracting different facial emotion of a person: Happy, anger, surprise, neutral. There is a degree for further upgrades and enhancements. Progressively effective approaches to incorporate different highlights and functionalities should, in any case, be investigated due to the lopsided nature of each element set. It is additionally seen that to improve the exactness of the arrangement framework the informational collection used to construct the grouping model could be expanded further. 


\section{REFERENCES}

Bhat, A. S., Amith, V. S., Prasad, N. S., \& Mohan, M. (2014). An Efficient Classification Algorithm For Music Mood Detection In Western and Hindi Music Using Audio Feature Extraction. 2014 Fifth International Conference on Signal and Image Processing, 359364. https://doi.org/10.1109/ICSIP.2014.63

Chew, L. W., Seng, K. P., Ang, L. M., Ramakonar, V., \& Gnanasegaran, A. (2011), Audio-Emotion Recognition System using Parallel Classifiers and Audio Feature Analyzer. In Proceedings of the 2011 Third International Conference on Computational Intelligence, Modelling \& Simulation (pp. 210-215), USA. IEEE. https://doi.org/10.1109/ CIMSim.2011.44

Deny, J., \& Sundararajan, M. (2014). Survey of Texture Analysis UsingHistogram in Image Processing. International Fournal of Applied Engineering Research, 9(26), 87378739. https://www.researchgate.net/publication/304091674_Survey_of_Texture_ Analysis_Using_Histogram_in_Image_Processing

Deny, J., \& Sundhararajan, M. (2015). Multi Modal Biometric Security for MANET Military Application-Face and Fingerprint. Fournal of Computational and Theoretical Nanoscience, 12(12), 5949-5953. https://www.researchgate.net/ publication/304380954_Multi_Modal_Biometric_Security_for_MANET_ Military_Application-Face_and_Fingerprint

Deny, J., Muthukumaran, E., Ramkumar, S., \& Kartheesawaran, S. (2018). Extraction Of Respiratory Signals And Motion Artifacts From PPG Signal Using Modified Multi Scale Principal Component Analysis. International fournal of Pure and Applied Mathematics, 119(12), 13719-13727. https://www.researchgate. net/publication/325465991_Extraction_Of_Respiratory_Signals_And_ Motion_Artifacts_From_PPG_Signal_Using_Modified_Multi_Scale_Principal_ Component_Analysis

Dureha, A. (2014). An Accurate Algorithm for Generating a Music laylist based on Facial Expressions. International Journal of Computer Applications, 100(9). https://pdfs. semanticscholar.org/312b/2566e315dd6e65bd42cfcbe4d919159de8a1.pdf 
Fan, X., Zhang, F., Wang, H., \& Lu, X. (2012). The System of Face Detection Based on OpenCV. In 24 th Chinese Control and Decision Conference (CCDC), Taiyuan, China. IEEE. https://doi.org/10.1109/CGDC.2012.6242980

Gilda, S., Zafar, H., Soni, G., \& Waghurdekar, K. (2017). Smart Music Player Integrating Facial Emotion Recognition and Music Mood Recommendation. In 2017 International Conference on Wireless Communications, Signal Processing and Networking (WiSPNET), Chennai, India. IEEE. https://doi.org/10.1109/WiSPNET.2017.8299738

Gossi, D., \& Gunes, M. H. (2016). Lyric-based music recommendation. In Cherifi H., Gonçalves B., Menezes R., Sinatra R. (eds.) Complex Networks VII. Studies in Computational Intelligence, vol. 644. Springer, Cham. https://doi.org/10.1007/978-3319-30569-1_23

Gupta, S. (2018). Facial emotion recognition in real-time and static images. In 2nd International Conference on Inventive Systems and Control (ICISC), Coimbatore, India. IEEE. https://doi.org/10.1109/ICISG.2018.8398861

Knyazev, B., Shvetsov, R., Efremova, N., \& Kuharenko, A. (2018). Leveraging large face recognition data foremotion classification. In 13th IEEE International Conference on Automatic Face $\mathcal{E}$ Gesture Recognition (FG 2018), Xi'an, China. IEEE. https://doi. org/10.1109/FG.2018.00109

Levi, G., \& Hassner, T. (201 1). Emotion Recognition in the Wild via Convolutional Neural Networks and Mapped Binary Patterns. In Proceedings ACM International Conference on Multimodal Interaction (ICMI), Seattle. https://doi.org/10.1 145/2818346.2830587

Muthukumaran, E., Deny, J., Perumal, B., Suseendran, G., \& Akila, D. (2015). A narrative Non-Invasive Diagnostic loom Based by the side of correlation of Nasal set Rhythm in addition to customary Three Radial Pulses Measurement. Fournal of Physics: Conference Series, 1228(1). https://iopscience.iop.org/ article/10.1088/1742-6596/1228/1/012075 
Sankaranarayanan, S., \& Deny, J. (2013). Securable Image Compression Using SPIHT Algorithm. International journal of Electronics and Communication Engineering \& Technology, $4(4)$, 96-100. https://fdocuments.in/document/securable-image-compressionusing-spiht-algorithm.html

Tzanetakisand, G., \& Cook, P. (2002). Musical Genre Classification of Audio Signals. IEEE Transactions on Speech and Audio Processing, 10(5). https://doi.org/10.1109/ TSA.2002.800560

Youssif, A. A. A., \& Wesam, A. A. A. (2011). Automatic Facial Expression Recognition System based on Geometric and Appearance Features. Computer and Information Science, 4(2), 115-124. https://doi.org/10.5539/cis.v4n2p115

Zeng, Z., Pantic, M., Roisman, G. I., \& Huang, T. S. (2009). A survey of affect recognition methods: Audio, visual, and spontaneousexpressions. IEEE Transactions on Pattern Analysis and Machine Intelligence, 31(1). https://doi.org/10.1109/TPAMI.2008.52 


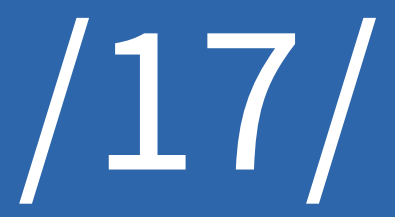




\section{INTELLIGENT GAS BOOKING AND LEAKAGE SYSTEM USING WIRELESS SENSOR NETWORKS}

Kalpana Murugam

Department of Electronics and Communication Engineering,

School of Electronics and Electrical Technology,

Kalasalingam Academy of Research and Education,

Krishnankoil, Virudhunagar Dt., (India).

E-mail: drmkalpanaece@gmail.com ORCID: https://orcid.org/0000-0002-5121-0468

\section{Citación sugerida:}

Murugam, K. (2020). Intelligent gas booking and leakage system using wireless sensor networks. 3C Tecnología. Glosas de innovación aplicadas a la pyme. Edición Especial, Marzo 2020, 273-285. http://doi. org/10.17993/3ctecno.2020.specialissue4.273-285

\section{Suggested citation:}

Murugam, K. (2020). Intelligent gas booking and leakage system using wireless sensor networks. 3C Tecnología. Glosas de innovación aplicadas a la pyme. Edición Especial, Marzo 2020, 273-285. http://doi. org/10.17993/3ctecno.2020.specialissue4.273-285 


\section{ABSTRACT}

Nowadays the technology is rapidly growing, and human life is becoming easier than the past. In our country maximum number of people is using LPG (Liquid Petroleum Gas) in several places like houses, shops and in many industries. To assemble of LPG is too diminutive. But it is very difficult to the illiterate people to understand and many of the people in our society is busy with their works, so that they can't know the status of the gas and if the booking of the LPG gets delay then it will be uncomfortable to the people. In order to overcome this problem, Intelligent Gas booking and Leakage Detection System (IGLS) is proposed. When the LPG gas gets minimum weight then GSM drive the memo to the owner and warns about the LPG gas is getting over, so that the owner tries to book the LPG in advance. Huge numbers of people are facing the gas leakage problem. In order to avoid this drawback, a gas sensor is utilized to sense the gas outflow and switch ON the buzzer and sends a message of outflow of the gas to warn the owner. Proposed system will be benefitted for society.

\section{KEYWORDS}

Gas sensor, Gas detector, Wireless Sensor networks, LCD display, Load cell, Buzzer, Arduino board. 


\section{INTRODUCTION}

LPG is known as Liquid Petroleum Gas. In Arpitha, Kiran, Gupta, and Duraiswamy (2016) consists of saturated propane and saturated butane mixture as well as unsaturated hydrocarbons. In LPG, Ethanethiol is added (Macker, Shukla, Dey, \& Agarwal, 2018). So that it becomes odourless gas, which will easily detect leakage of gas. In our country (Varma, Prabhakar, \& Jayavel, 2017) there are approximately 40 crore people are using LPG which is nearly $45 \%$ of our population. In Shinde, Patil, and Patil (2012), and Mahalingam, Naayagi, and Mastorakis (2012), LPG gas, which is commercially used in domestic area for heating, cooking, shops and industrial purpose, etc., LPG has highly flammable because of the mixture of propane and butane. In Priya, Surekha, Preethi, Devika, and Dhivya (2014) a cylinder is to be found that it is loaded, which will measure the weight of the cylinder. In Ramya and Palaniappan (2012) inserted a minimum weight of the cylinder according to the level of a gas.

\section{RELATED WORK}

In Shyamaladevi, Rajaramya, Rajasekar, and Ashok (2014) proposed a structure that will make the whole LPG compartment booking system robotized was excluded human intervention. This framework ceaselessly decides the greatness of the chamber drive notice to the insisted LPG agent with the target that they can pass on the LPG chamber in time.

In Cheung, Lin, and Lin (2018) consolidates WSN into an astounding structure which enables the structure site to ostensibly screen the prosperity status by methods for a spatial, concealed interface and empty any perilous gas normally. In Dewi and Somantri (2018) were proposed the inspiration driving this structure is to recognize gas spillage, murder it, and thwart the impact. WSN framework works were reliant on gas sensor MQ-6 and remote module Bluetooth HC-05. Impact neutralizing activity system works reliant on the alarm/ringer, exhaust fan, and customized gas controller.

In Hema, Murugan, and Chitra (2013) the light was unmistakable tangibility and common confinements of different gas identifying developments, experts have been managing different circumstances with overhauled frameworks. In this, they were analyzed the overview of progressed enhancement in leakage of gas recognizing. In Kalaivanan and 
Manoharan (2016) were the essential objectives of the endeavor is to gather a Gas spillage discoverer using LPG gas sensor and furthermore it was interface with IoT using ESP module for security. A solenoid is set such a way, that at whatever point there is a sign, it will close the pipe to stop the movement of gas.

In Padmapriya and Kamini (2013) the structure also is used for other application in the business or plant that depends upon LPG and vaporous oil in their action this endeavor is used to screen relentless load of the LPG chamber. In our country customarily that people don't know decisively the status of the chamber and there is a deferment in lighting up gas office. To keep far from such conditions have completed the endeavor called "Customized Gas Booking Using Embedded System with Safety Guards". In this endeavor used weight cell as a weight sensor. MQ6 as an LPG spillage sensor which will recognize the spillage of LPG and offers security to people. The gas office gets the solicitation of new chamber and owner got the messages regarding the status. In paper (Apeh, Erameh, \& Iruansi, 2014) were smoke sensor was used to recognize gas spillage in the home. In case any gas spillage recognized subsequently, it will send SMS to the fire station. GSM is a champion among the most cell frameworks used in India. In our endeavor, we have used weight cell to screen the hold up of the LPG gas routinely. In Bangali and Shaligram (2013) proposes the Internet of Things is considered as the third surge of information development straightforwardly after Internet and flexible correspondence sort out, which is depicted by progressively complete interoperability and learning. By this creative progress, it engages us to screen the activities through our propelled cell phones, to share information.

In Potadar, Salvi, Sathe, and Chavan (2015) proposes the system will recognize the spillage and exhort the client about the gas spillage by SMS Audio yield is moreover made on speaker. As a bit of emergency, the system will immediately temperament executioner the control valve with the help of stepper motor to evade a mishap. The additional good position of the system is that it constantly screens the component of the LPG present in the chamber using load cell and demonstrate the weight always. The programmed gas booking framework will really discard call stopping or dialing such countless numbers while booking the gas chamber. 
In Ravichandran (2017) were LPG will finish without instructing us it can make an inconvenient condition for cooking, etc. Our arrangement relies upon ARM controller, it can pursue LPG void continually in case LPG is close culmination or at void measurement, by then, it can alert us by sending SMS to LPG Agency for mentioning the LPG chamber. In Imade, Rajmanes, Gavali, and Nayakwadi (2018) were analyzed, the fundamental stress of any assignment has not been left impeccable by IoT. In Khan, Prince, Dewangan, and Rathore (2014), Gas Leakages in open or shut districts can show to be dangerous and savage. The customary Gas Leakage Detector Systems have various phenomenal precision; disregard to perceive two or three segments in the field of disturbing the all-inclusive community about the spillage. In Jolhe, Potdukhe, and Gawai (2013) in the unlikely event that LPG is going to complete without teaching us, it can make an irksome condition for cooking, etc. Our structure relies upon the microcontroller, it can pursue LPG void continually in case LPG is especially near completion or at void measurement, by then it can alert us by sending SMS to the owner and it can in like manner move the message to LPG Agency for mentioning the LPG chamber.

\section{PROPOSED SYSTEM}

\subsection{INTELLIGENT GAS BOOKING AND LEAKAGE DETECTION SYSTEM (IGLS)}

Gas leakage means if the gas is transferred from one pipe to another pipe or other container of natural gas or another gas product may not be present. Leaks are very dangerous because a small leak can gradually produce an explosive concentration of gas. This is the main drawback of traditional gas leakage system. In order to overcome the above problem, this work proposes Intelligent Gas booking and Leakage Detection System (IGLS).

When the LPG gets to the base weight, the GSM sends the message to the proprietor by the assistance of Arduino. Arduino is accustomed to sending the message to the client with the assistance of GSM. The LCD shows the heaviness of the chamber consistently. This program is created to send the message through GSM to the given number of the client. The gas sensor MQ6 to detect the gas around the place, after that microcontroller automatically switch ON the signal and sends the message to the proprietor. 
The message sent to the fixed number automatically and access remotely with the assistance of GSM. At the point when the heaviness of the gas chamber gets least; GSM sends the message to the proprietor and cautions the proprietor about the leakage of the gas.

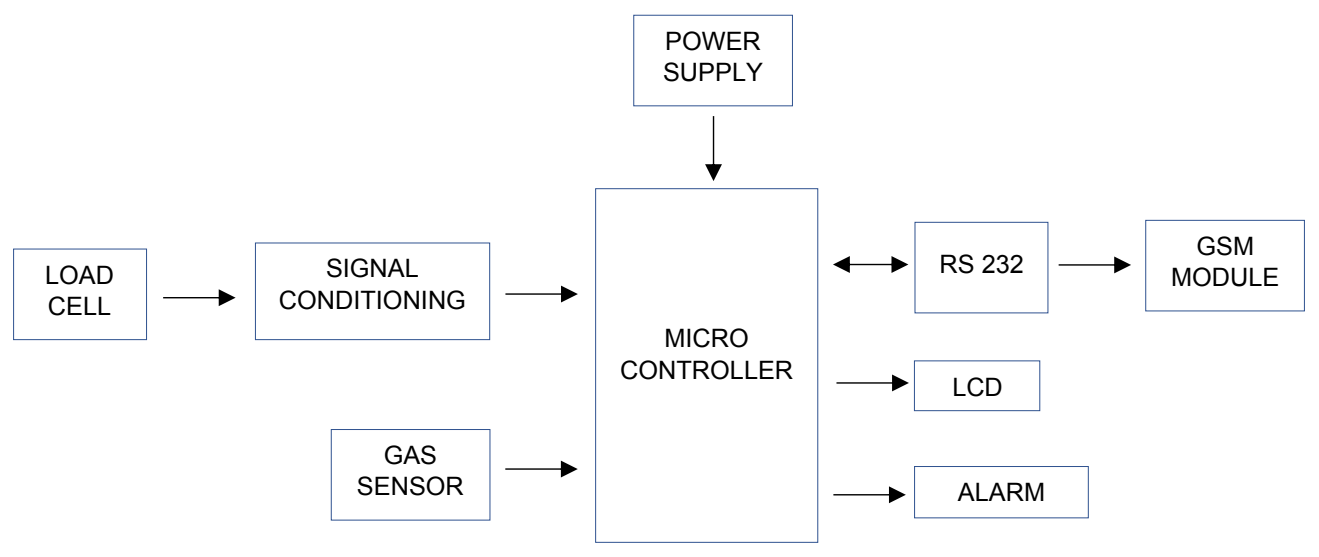

Graphic 1. BLOCK DIAGRAM.

\section{METHODOLOGY}

The main aim of this system is to estimate the GAS level and leakage of the gas from the room or an industry. The leakage of the gas will be estimated with the help of IGLS system using MQ6 gas sensor. This gas sensor senses the gas and sends the message to the user with the help of GSM module. Liquefied Petroleum Gas (LPG) sensor is simple and easy to detect the gas level. Gas sensor has fast response and high sensitivity module. Liquefied Petroleum Gas (LPG) sensor is simple and easy to detect the gas level. Gas sensor has fast response and high sensitivity.

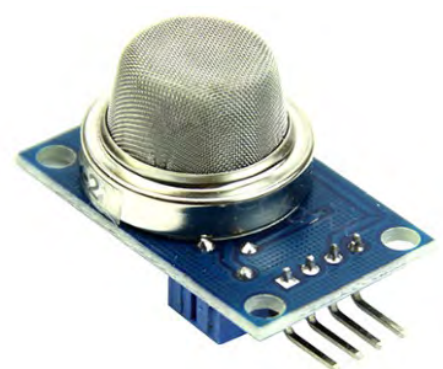

Graphic 2. PIR Sensor. 
Table 1. Specification of MQ6 sensor.

\begin{tabular}{|c|c|c|c|}
\hline \multicolumn{3}{|c|}{ Model No. } & MQ-6 \\
\hline \multicolumn{3}{|c|}{ Sensor Type } & Semiconductor \\
\hline \multicolumn{3}{|c|}{ Standard Encapsulation } & Bakelite (Black Bakelite) \\
\hline \multicolumn{3}{|c|}{ Detection Gas } & Isobutane, Butane, LPG \\
\hline \multicolumn{3}{|c|}{ Concentration } & $\begin{array}{c}300-10000 \text { ppm } \\
\text { (Butane, Propane, LPG) }\end{array}$ \\
\hline \multirow{3}{*}{ Circuit } & Loop Voltage & $\mathrm{V}_{\mathrm{c}}$ & $\leq 24 \mathrm{~V} \quad \mathrm{DC}$ \\
\hline & Heater Voltage & $\mathrm{V}_{\mathrm{H}}$ & $5.0 \mathrm{~V} \pm 0.2 \mathrm{~V}$ ACorDC \\
\hline & Load Resistance & $\mathrm{R}_{\mathrm{L}}$ & Adjustable \\
\hline \multirow{5}{*}{ Character } & Heater Resistance & $\mathrm{R}_{\mathrm{H}}$ & $31 \Omega \pm 3 \Omega$ (Room Tem.) \\
\hline & Heater consumption & $\mathrm{P}_{\mathrm{H}}$ & $\leq 900 \mathrm{~mW}$ \\
\hline & Sensing Resistance & $\mathrm{R}_{\mathrm{s}}$ & $2 \mathrm{~K} \Omega-20 \mathrm{~K} \Omega$ (in 2000ppm C3H8) \\
\hline & Sensitivity & S & Rs (in air) / Rs (1000ppm C4H10) $\geq 5$ \\
\hline & Slope & $\alpha$ & $\leq 0.6$ (R2000ppm / R1000ppm LPG) \\
\hline \multirow{3}{*}{ Condition } & \multicolumn{2}{|c|}{ Tem. Humidity } & $20^{\circ} \mathrm{C} \pm 2^{\circ} \mathrm{C} ; 65 \% \pm 5 \% \mathrm{RH}$ \\
\hline & \multicolumn{2}{|c|}{ Standard test circuit } & $\mathrm{V}_{\mathrm{c}}: 5.0 \mathrm{~V} \pm 0.1 \mathrm{~V} ; \mathrm{V}_{\mathrm{H}}: 5.0 \mathrm{~V} \pm 0.1 \mathrm{~V}$ \\
\hline & \multicolumn{2}{|c|}{ Preheat time } & Over 48 hours \\
\hline
\end{tabular}

\section{WORKING PRINCIPLE}

The working standard of this system has appeared in the square outline in Figure 1. The Arduino board is assuming an indispensable job which is utilized to quantify the parameters of gas and weight. Every one of the parts is associated with the Arduino board. Arduino gets the messages to the purchaser about the gas chamber like gas spillage and gas weight of the chamber. At the point when the gas is spilling from the gas chamber, the gas sensor will detect the gas spillage and after that sends the data to the Arduino then the Arduino will check the code and sends the data to the GSM module and ringer. The GSM module will send the short message too private the client to realize the gas is spilling and the ringer will make the noisy sound that underwear that gas is spilling. This work will screen with the assistance of GSM ceaselessly if the gas weight is low as for the given load in the code. The gas weight is recognized by the heap cell. The heap cell checks the heaviness of the chamber if the heaviness of the chamber is lower than the given code it will send the data to the Arduino board. It will give the direction to the GSM module to send the message to the customer that the gas weight is low and it starts the client to book the gas chamber. By utilizing the LCD show the weight and the gas spillage estimations of the chamber are shown routinely. 


\section{RESULT AND DISCUSSION}

Intelligent Gas booking and Leakage Detection System is implemented in real time. The gas weight and the gas spillage are estimated by utilizing IGLS, the estimated gas values are showed in the Liquid Crystal Display (LCD). The gas present in the room air before the gas spillage having the level is $0 \mathrm{ppm}$, which was appeared in the LCD. When the gas was spilled from the gas chamber the gas sensor will detect the gas in the room air. The GSM module will send the message to the individual proprietor of the user where the portable number was in the GSM module and sends the message "GAS WAS LEAKING". On and off messages send to the controller when the gas was not spilling from the gas chamber. Burden cell will detect the heaviness of the gas chamber. On and off message shows that the heaviness of the chamber is not exactly the edge weight, at that point, it will send the message to the user that the "GAS CYLINDER WEIGHT IS LOW BOOK THE GAS CYLINDER" was sent to the versatile number present in the GSM module. This system will be benefit able for society.

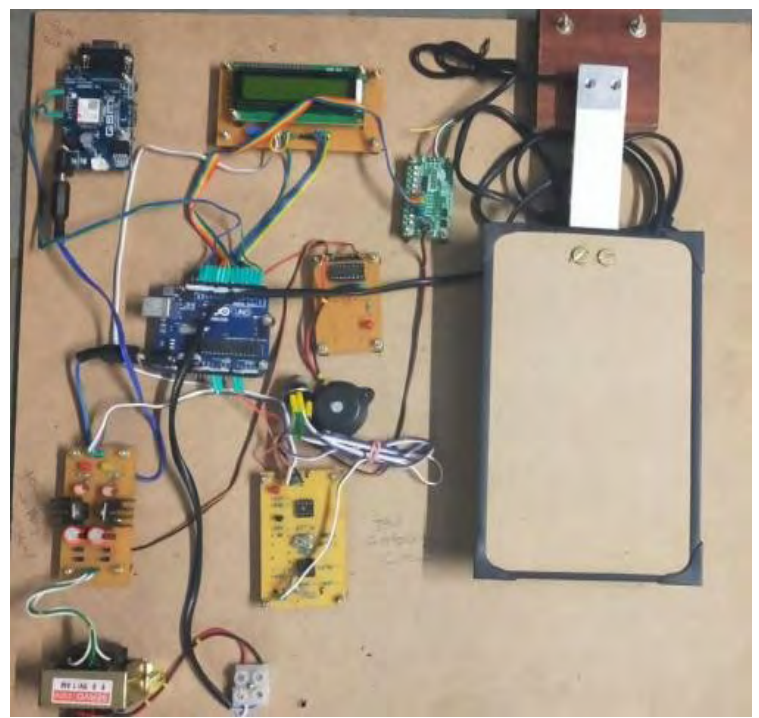

Graphic 3. Output for gas booking and leakage system System.

\section{CONCLUSION}

This project is based on the Intelligent gas booking system using wireless sensor networks and it is working well, and the main parameters of the Intelligent gas booking system is 
based on the safety purpose of the of day today life everyone are using the LPG gas for cooking and other purposes there is no safety in the place. This project will work nicely in any complex stages. During the design as well as the construction on it because to avoid the hiccups at the final stage. The layout was prepared with almost care to avoid wrong things in the circuit is made as simple as possible to understand easily to our knowledge. The components also taken care about these performance and cost effectiveness. It was interesting while preparing the project and some difficult at some stages it will be enthusiastic and easy to do the work on the project. This project is benefitted for society. 


\section{REFERENCES}

Apeh, S. T., Erameh, K. B., \& Iruansi, U. (2014). Design and Development of Kitchen Gas Leakage Detection and Automatic Gas Shut off System. Fournal of Emerging Trends in Engineering and Applied Sciences (JETEAS), 5(3), 222-228. http://jeteas. scholarlinkresearch.com/articles/Design \%20and\%20Development $\% 20$ of\%20 Kitchen\%20Gas\%20Leakage.pdf

Arpitha, T., Kiran, D., Gupta, V. S., \& Duraiswamy, P. (2016). FPGA-GSM based gas leakage detection system. IEEE Annual India Conference (INDICON), Bangalore, India, pp.1-4. IEEE. https://doi.org/10.1109/INDICON.2016.7838952

Bangali, J., \& Shaligram, A. (2013). Design and Implementation of Security Systems for Smart Home based on GSM technology. International Fournal of Smart Home, 7(6), 201-208. http://dx.doi.org/10.14257/ijsh.2013.7.6.19

Cheung, W. F., Lin, T. H., \& Lin, Y. C. (2018). A real-time construction safety monitoring system for hazardous gas integrating wireless sensor network and building information modeling technologies. Sensors, 18(2), 436. https://doi.org/10.3390/s18020436

Dewi, L., \& Somantri, Y. (2018). Wireless Sensor Network on LPG Gas Leak Detection and Automatic Gas Regulator System Using Arduino. In IOP Conference Series: Materials Science and Engineering, 384(1), 012064. https://doi.org/10.1088/1757899X/384/1/012064

Hema, L. K., Murugan, D., \& Chitra, M. (2013). WSN based Smart system for detection of LPG and Combustible gases. In National Conference on Architecture, Software systems and Green computing-2013. https://pdfs.semanticscholar. org/304f/541754fle24f8ed6fdf4c2750a1c771173aa.pdf

Imade, S., Rajmanes, P., Gavali, A., \& Nayakwadi, P. V. N. (2018). Gas leakage detection and smart alerting system using IOT. International fournal of Innovative research \& studies, 2(II). https://www.pramanaresearch.org/gallery/22.\%20feb\%20ijirs \%20 $-\% 20 d 539 . p d f$ 
Jolhe, B. D., Potdukhe, P. A., \& Gawai, N. S. (2013). Automatic LPG booking, leakage detection and real time gas measurement monitoring system. International Journal of Engineering Research \& Technology (IfERT), 2(4), 1192-1195. https://www. ijert.org/research/automatic-lpg-booking-leakage-detection-and-real-time-gasmeasurement-monitoring-system-IJERTV2IS4612.pdf

Kalaivanan, S., \& Manoharan, S. (2016). Monitoring and Controlling of Smart Homes using IoT and Low Power Wireless Technology. Indian fournal of Science and Technology, 9(31), 1-9. https://doi.org/10.17485/ijst/2016/v9i31/92701

Khan, A., Prince, N. K., Dewangan, S. K., \& Rathore, P. S. (2014). GSM based automatic LPG ordering system with leakage alert. IfRET: International fournal of Research in Engineering and Technology, 3. https:/ /www.academia.edu/8034393/GSM_ BASED_AUTOMATIC_LPG_ORDERING_SYSTEM_WITH_LEAKAGE_ ALERT

Macker, A., Shukla, A. K., Dey, S., \& Agarwal, J. (2018). ARDUINO Based LPG Gas Monitoring Automatic Cylinder Booking with Alert System. In 2nd International Conference on Trends in Electronics and Informatics (ICOEI), Tirunelveli, India. IEEE. 12091212. https://doi.org/10.1109/ICOEI.2018.8553840

Mahalingam, A., Naayagi, R. T., \& Mastorakis, N. E. (2012). Design and implementation of an economic gas leakage detector. Recent Researches in Applications of Electrical and Computer Engineering, 20-24. http://www.wseas.us/e-library/ conferences/2012/Vouliagmeni/ACA/ACA-02.pdf

Padmapriya, R., \& Kamini, E. (2013). Automatic LPG Booking, Leakage Detection and a Real Time LPG Measurement Monitoring System. https:/ / docplayer.net/8700630-Automaticlpg-booking-leakage-detection-and-a-real-time-lpg-measurement-monitoringsystem.html

Potadar, M. P., Salvi, P. S., Sathe, R. B., \& Chavan, P. S. (2015). LPG Leakage Detection and Automatic Gas Cylinder Booking System. International fournal of Engineering Research. 
Priya,K.P.,Surekha,M.,Preethi,R.,Devika,T., \&Dhivya,N.(2014).Smartgas cylinder using embedded system. International Fournal of Innovative Research in Electrical, Electronics, Instrumentation and Control Engineering (IFIREEICE), 2(2), 958-962. http://citeseerx.ist. psu.edu/viewdoc/download?doi=10.1.1.428.5116\&rep=rep 1\&type=pdf

Ramya, V., \& Palaniappan, B. (2012). Embedded system for Hazardous Gas detection and Alerting. International Fournal of Distributed and Parallel Systems (IFDPS), 3(3), 287300. https://doi.org/10.5121/ijdps. 2012.3324

Ravichandran, S. (2017). Cloud Connected Smart Gas Cylinder Platform Senses LPG Gas Leakage Using IoT Application. International fournal of MC Square Scientific Research (IFMSR), 9(1), 324-330. https://pdfs.semanticscholar.org/ bc5b/3982690389a19060c9dac08eb0df42ab3fec.pdf

Shinde, S., Patil, S. B., \& Patil, A. J. (2012). Development of movable gas tanker leakage detection using wireless sensor network based on embedded system. International Journal of Engineering Research and Application (IfTERA), 2(6), 1180-1183. http:// citeseerx.ist.psu.edu/viewdoc/download?doi=10.1.1.415.33\&rep=rep $1 \&$ type=pdf

Shyamaladevi, S., Rajaramya, V. G., Rajasekar, P., \& Ashok, P. S. (2014). Arm7 based automated high performance system for LPG refill booking \& leakage detection. International journal of engineering research, science and technology (IfERST), 3(2).

Varma, A., Prabhakar, S., \& Jayavel, K. (2017). Gas Leakage Detection and Smart Alerting and prediction using IoT. In 2nd International Conference on Computing and Communications Technologies (ICCCT), Chennai, India, pp. 327-333. IEEE. https://doi. org/10.1109/ICCGT2.2017.7972304 


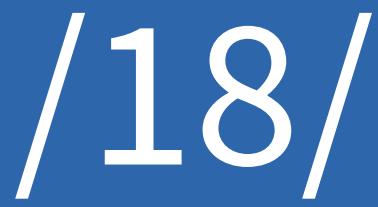




\section{SMART DRIVING SYSTEM WITH AUTOMATIC DRIVER ALERT AND BRAKING MECHANISM}

P. B. Dhanusha

Department of Electronics and communication Engineering, SAINTGITS College of Engineering Kottayam, Kerala, (India). E-mail: dhanusha.pb@saintgits.org ORCID: https://orcid.org/0000-0001-7375-168X

\section{A. Lakshmi}

Department of Electronics and Communication Engineering Kalasalingam

Academy of Research and Education Tamil Nadu, (India). E-mail: lakshmi@klu.ac.in ORCID: http://orcid.org/0000-0002-6744-7048

\section{K. Saravanan}

Department of Electronics and Communication Engineering, SAINTGITS College of Engineering Kottayam, Kerala, (India). E-mail: saravanan.k@saintgits.org ORCID: https://orcid.org/0000-0002-6160-3601

\section{Gitación sugerida:}

Dhanusha, P. B., Lakshmi, A., y Saravanan, K. (2020). Smart driving system with automatic driver alert and braking mechanism. $3 C$ Tecnología. Glosas de innovación aplicadas a la pyme. Edición Especial, Marzo 2020, 287-299. http://doi.org/10.17993/3ctecno.2020.specialissue4.287-299

\section{Suggested citation:}

Dhanusha, P. B., Lakshmi, A., \& Saravanan, K. (2020). Smart driving system with automatic driver alert and braking mechanism. 3C Tecnología. Glosas de innovación aplicadas a la pyme. Edición Especial, Marzo 2020, 287-299. http://doi.org/10.17993/3ctecno.2020.specialissue4.287-299 


\section{ABSTRACT}

Driving is one of the most important job for almost all people. Person use their vehicle to travel from one place to other. The count of automobiles is increasing every day. It increases the risk to accident. Currently, percentage numbers of accident are increasing drastically. One of the main reason for accident is the failure in concentration of the driver due to which he/she may fall asleep or sometimes due to the delay for applying the brake. A new system is developed that can solve these problems where an alert is given to the people present inside the vehicle to indicate that the driver is falling asleep and a co- system which can automatically stop the vehicle even if the driver may not brake manually due to obstacles. Our aim is to make a smart driving system with automatic waking alert and automatic braking system to ensure the safety of driving.

\section{KEYWORDS}

Smart, Automatic, Braking. 


\section{INTRODUCTION}

"Driving to save lives, time, and money in spite of the conditions around you and the actions of others" - one of the quotes for protective Driving.

Accidents are happening due to improper driving. The main causes for accidents are the drowsiness of the driver or unaware of surroundings. Driver drowsiness is found as a very important fact in the automobile accidents. It was observed that $20 \%$ of vehicle accidents occurs due to the increased drowsiness. We know that life that lost can't be re-winded. Accidents due to this reason can be avoided with the help of advanced electronic technology to an extent (Fisher \& Talwar, 2013).

Studies shows that drowsiness is one of the important reason for accidents and it can impair the brain of a human being as much as an alcohol can. In a survey it was found that, twenty three percentage of people have fallen asleep during driving. According to Department of Transportation, United States, the tendency for fallen asleep during driving for male is twice as much as female drivers. As claimed by the National Highway Traffic Safety Administration, drowsiness is the only factor in more than 100,000 accidents, causing 1,550 deaths and 40,000 bruise annually in USA.

The chances of accidents can be reduced by the effective use of advanced electronics technology. If all the vehicles are implanted with an automated security system such that the system provides good security to driver along with an alarm, we can decrease the chances of accidents. The percentage of accidents is increasing every day since the amount of vehicles is also increasing. The main reason for the accident is due to the delay caused by the driver to hit the brake. In order to stop this type of accidents, a system with automatic braking can be implemented. (Niehaus \& Stengel, 1991) The proposed system gives an automatic driver alert and braking mechanism by which the rate of accidents can be reduced. The important part of the system is a brain wave sensor. The sensor detects the drowsiness of the human being by sensing the brain waves. These waves are processed, and an alarm is operated if needed. The system also provides an additional facility of automatic braking if there is a delay in applying the brake by the driver. This system measures brainwave strength using brainwave sensor and give waking alert if the driver falls asleep. The system also gives automatic braking assistance using ultrasonic sensor. 


\section{MATERIALS AND METHODS}

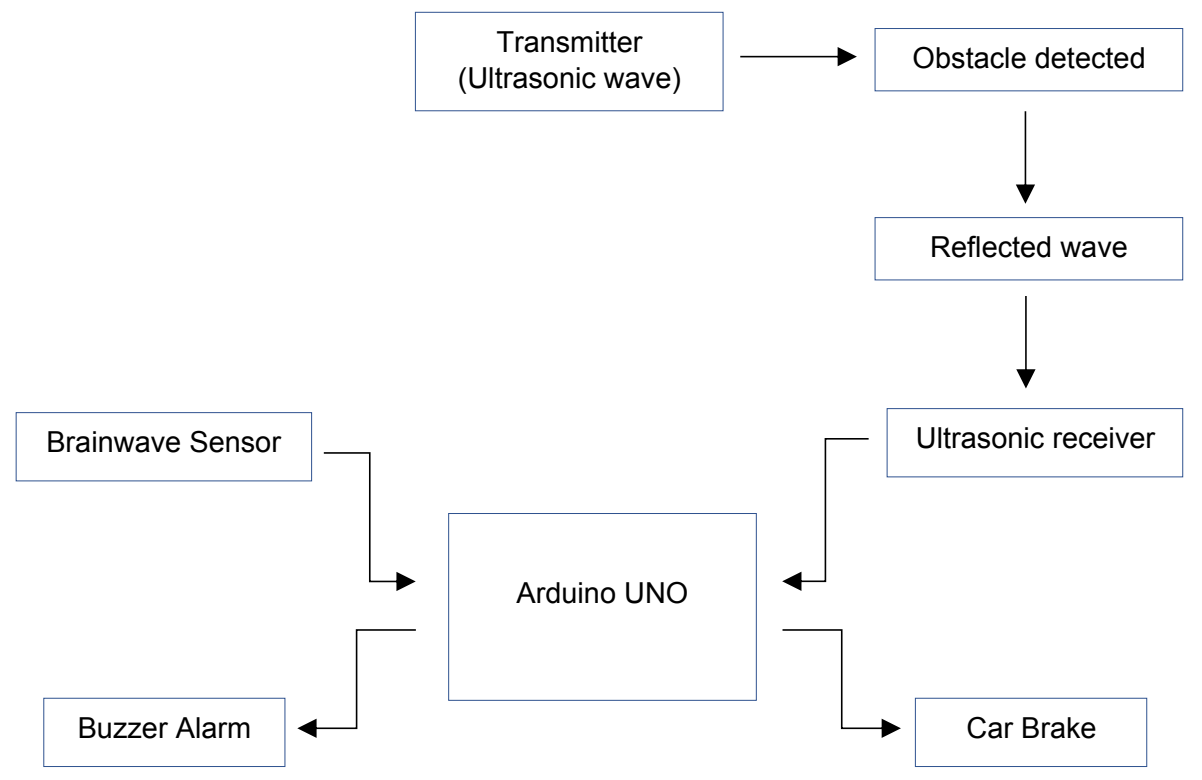

Figure 1. Overall block diagram.

The brainwaves are measured using the brainwave sensor and is analysed by the Arduino program. When the certain waves (mainly gamma) falls under a threshold value we can say the driver is going to fall asleep. When the threshold level is reached, we will give a buzzer alarm to wake the driver. The smart system consists of a sender and receiver which sends the ultrasonic waves and also receives. (Wang, Zeng, \& Yang, 2006) The ultrasonic signal emitter is fixed at the front of the automobile, which emits ultrasonic waves in a preset distance at the front of the automobile. Ultrasonic signal collector is also fixed in front of the car, which receives the ultrasonic wave which is reflected from the obstacle. The distance between the car and the barrier is measured by analyzing the received ultrasonic signal. Using the program which is programmed for automatic braking will control the braking system according to this distance. Brake is applied automatically to avoid forward collision.

\section{HARDWARE REQUIRMENT}

Arduino UNO, Mind-flex Brainwave sensor, Ultrasonic sensor, LEDs, Battery Operated motors, Buzzer, Bread board, Dot board, connecting wire 


\section{SOFTWARE REQUIREMENT}

Arduino programming $(\mathrm{c}++)$, Fritzing.

\section{WORKING OF CIRCUIT}

The important part of the smart system is a sender and receiver which sends the ultrasonic waves and also receives. The ultrasonic signal emitter is fixed at the front of the vehicle, which emits ultrasonic waves in a preset distance at the front of the vehicle. Ultrasonic signal collector is also fixed at the front of the car, which receives the ultrasonic signal reflected from the obstacle. The car and the barrier separation are measured by analyzing the obtained ultrasonic signal. The reflected wave is measured so that the separation between the automobile and the obstacle is obtained. This distance is analysed using the Arduino program and based on this signals are given to motor shield. According to these signals motor is controlled. Here we take $30 \mathrm{~cm}$ as alerting point, in which led will turned on and we take $20 \mathrm{~cm}$ as braking point in which automatically brake is applied. The brainwaves are measured using the Mindflex brainwave sensor and is analysed by the Arduino program. When the certain waves (mainly gamma) falls under a threshold value we can say the driver is going to fall asleep. When the threshold level is reached, we will give a buzzer alarm to wake the driver.

\section{SMART DRIVING}

The main idea is to merge the above two ideas and produce a smart driving system for driving especially at night. Because the chances of falling asleep and chances of collision with obstacles are very high compared to day. The block diagram indicating the same.

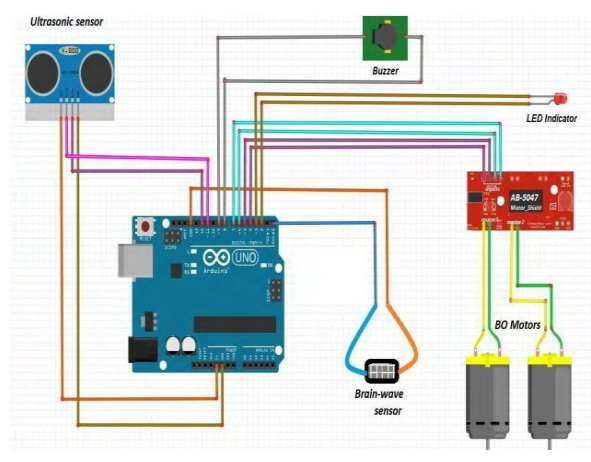

Figure 2. Circuit diagram. 


\section{COMPONENT DESGRIPTION}

\section{$\underline{\text { ARDUINO UNO }}$}

The sensing of the obstacle is done using Arduino. It is an open source companies that develops microcontroller kits which can senses/detect objects in the real world for different applications.

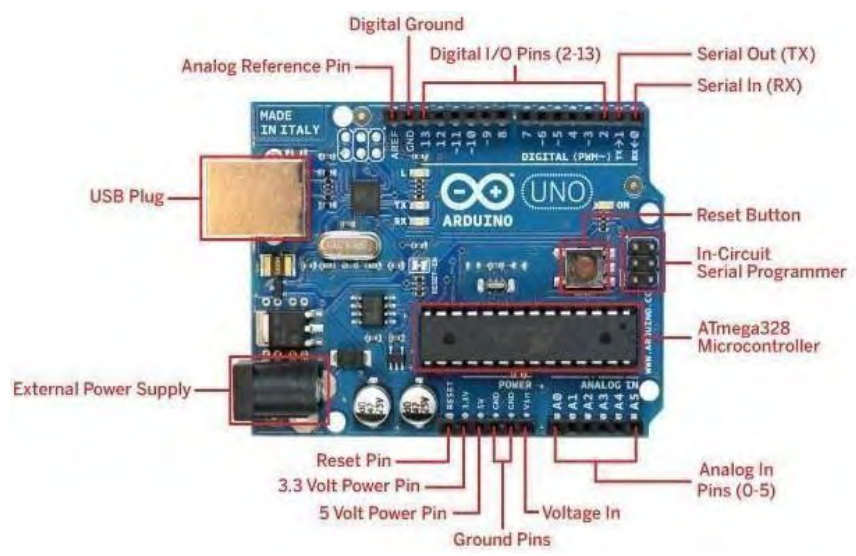

Figure 3. ARDUINO UNO.

It is possible to design Arduino boards by using different types of processors and controllers based on the application. It has many digital input and digital output pins by which the kit must be interfaced to other boards and electronic circuits. Some kits provide Universal Serial Bus (USB) connectors through which we can load programs from the personal computers. The integrated chip is generally programmed using $\mathrm{C}$ or $\mathrm{C}++$.

This work makes use of the Arduino board to interface all the components and they are run by a program compiled by Arduino software. The microcontroller used in Arduino Uno is ATmega 328P. Arduino Uno is the commonly used kit of the Arduino family. Arduino Uno has fourteen digital I/O pins, six analog input pins, a $16 \mathrm{MHz}$ quartz crystal, a power jack and a USB connection.

\section{ULTRASONIC SENSOR - HC-SR04}

The ultrasonic sensor used in the proposed system is HC-SR04. This sensor gives up to $400 \mathrm{~cm}$ of measurement with $3 \mathrm{~mm}$ ranging accuracy. The different modules included are an ultrasonic receiver, transmitter and a control circuitry. 


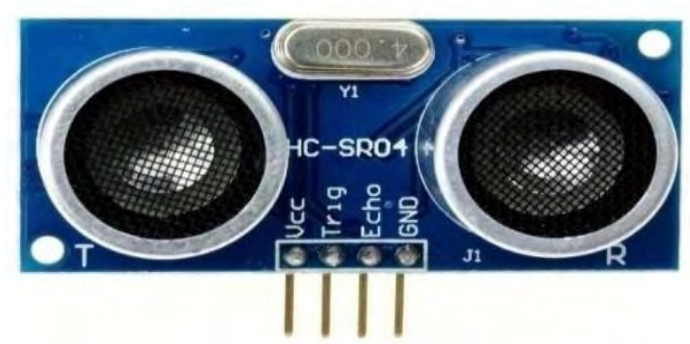

Figure 4. Ultrasonic sensor HC-SR04.

By analyzing the time delay between transmitted and received signal ofthe sensor, the range can be calculated.
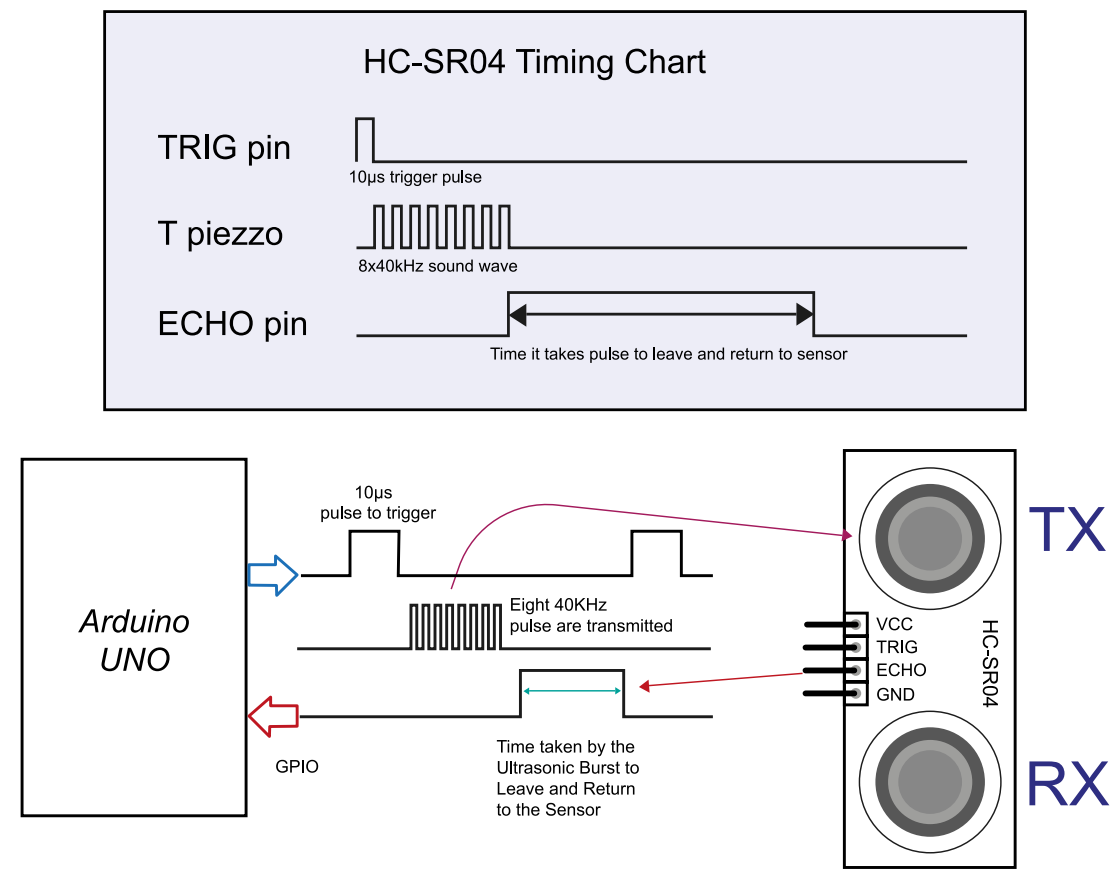

Figure 5. System Overview.

\section{BRAINWAVE SENSOR}

A sensor which can detect Brainwaves is known as a brainwave sensor. It will transform these brainwaves into electrical signals which can be used for the analysis. The figure shows a 'Mindflex' brainwave sensor which can detect brainwaves. This sensor can detect delta, theta, alpha, beta and Gamma waves of the brain. An electrode in the sensor have direct contact with the forehead of the person using the sensor which can detect brainwaves. 


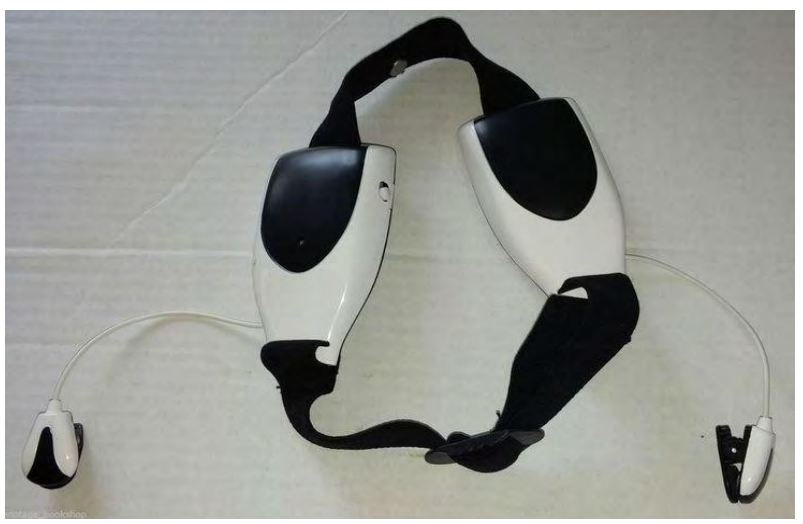

Figure 6. Brainwave sensor.

\section{BUZZER}

The signaling device used in this project is a buzzer. A signaling device has many applications like in automobiles, household appliances etc. A buzzer includes switches or sensors, which are controlled by a control unit that checks if and which button was pushed or a present time has lapsed, and blinks light on the appropriate button or control panel and sounds a warning in the form of beeping sound.

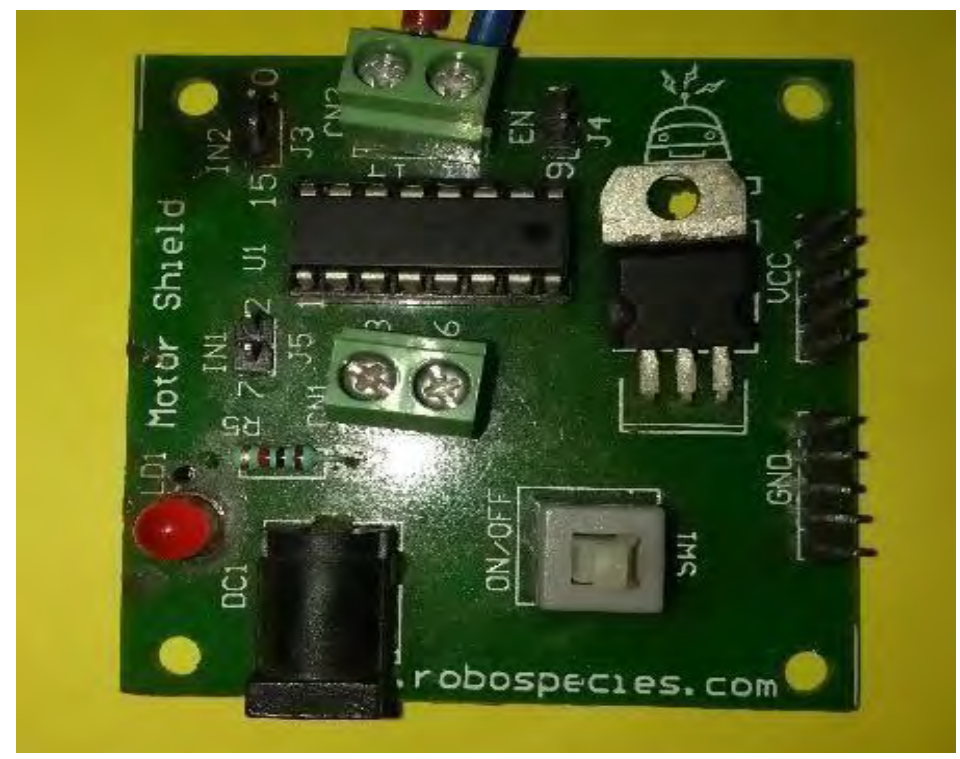

Figure 7. Arduino Motor Shield. 


\section{$\underline{\text { MOTOR SHEILD }}$}

For controlling the DC motors we are using an Arduino Motor Shield which is developed to control relays, DC motors, solenoids, and stepping motors. By using the Arduino board control of two DC motor is possible. Here WE are using AB 5407 Motor shield for Arduino.

\section{BRAINWAVES}

Brain wave sensor is placed on the scalp, which senses the brain waves. According to the condition of our mind the frequencies of the brain waves will be varied. A low frequency signal indicates tiredness. For hyper alert condition the brain waves will have high frequency. Brain waves are divided into different types based on the frequency (Mostafa, Mustapha, Hazeem, Khaleefah, \& Mohammed, 2018).

Different types of brainwaves:

\section{INFRA-LOW WAVES}

Infra-Low brainwaves are thought to be the basic cortical rhythms that underlie our higher brain functions. Very little is known about infra-low brainwaves. They come under frequency less than $0.5 \mathrm{~Hz}$. They appear to take a major role in brain timing and network function.

\section{DELTA WAVES}

Frequency of the delta waves ranges from $0.5 \mathrm{~Hz}-3 \mathrm{~Hz}$. They are slow brainwaves with low frequency and highly penetrating in nature. Delta waves are produced during meditation or dreamless sleep.

\section{THETA WAVES}

Theta waves come under the frequency range 3 to $8 \mathrm{~Hz}$. Theta waves are generated during sleep. Theta waves generally gives information about learning and memory.

\section{$\underline{\text { ALPHA WAVES }}$}

The frequency of alpha waves ranges from 8 to $12 \mathrm{~Hz}$. Alpha brainwaves are generated during quietly flowing thoughts. Alpha is the resting state for the brain. Alpha brainwaves aim overall mental coordination, learning, calmness, mind/body integration and alertness. 


\section{BETA WAVES}

The frequency range of Beta waves is from 12 to $38 \mathrm{~Hz}$. Beta waves are generated when we are alert and attentive.

\section{GAMMA WAVES}

Brain waves which comes under the frequency range 38 to $42 \mathrm{~Hz}$ is called gamma waves. Gamma brainwaves are the fastest of brain waves. It is speculated that Gamma rhythms modulate perception and consciousness, and that a greater presence of Gamma relates to expanded consciousness and spiritual emergence.

\section{ARDUINO SOFTWARE DESGRIPTION}

In this work the platform used is an Arduino integrated development environment also known as IDE, in which the programming is written in Java. IDE provides different features like text cutting, searching, pasting, replacing text, automatic indenting, and syntax highlighting. The programs are compiled and uploaded to the Arduino kit using a very simple one click mechanism. The other important highlights of IDE include a message area, a text console, a toolbar with buttons etc.

The audio programs written for IDE are known as sketch. This sketches are saved as text files in the development computer with the extension as .ino. The languages supported by Arduino IDE are $\mathrm{C}$ and $\mathrm{C}++$ using special rules of code structuring.

\section{CONCLUSION}

A practical system (Automatic waking alert) which could detect whether the driver is going to fall asleep or the concentration level of the driver is too low is produced, it also gives alarm when the above situations occurs. An automatic braking system which could automatically apply brake if the driver takes too much time to manually apply the brake is also produced as part of this work.

We believe this system will reduce the chances of road accidents and ensure safe driving. 


\section{REFERENCES}

Borraz, R., Navarro, P. J., Fernández, G., \& Alcover, P. M. (2018). Cloud Incubator Car: A Reliable Platform for Autonomous. Driving. Applied Sciences, 8(2), 303. https:/ / doi.org/10.3390/app8020303

Fisher \& Talwar. (2013). Car Accident Statistics 2013. https://www.fishertalwar.com/caraccident-statistics/

John, S., \& Pedro, J. O. (2013). A comparative study of two control schemes for anti-lock braking systems. In 9th Asian Control Conference (ASCC), 1-6. https://doi.org/10.1109/ ASCG.2013.6606061

Lexus. (2017). Lexus Outlines Advanced Active Safety Technologies for All-Nere LS. https:// newsroom.lexus.eu/lexus-outlines-advanced-active-safety-technologies-for-all-newls/ (accessed on 20 December 2018).

Mi, G., Lin, H., \& Zhang, Y. (2009). Iterative learning control of antilock braking of electric and hybrid vehicle. IEEE Transactions on Vehicular Technology, 54(2), 486 -494. https://doi.org/10.1109/TVT.2004.841552

Modi, S., Chesnakov, D., Zhang, W. C., Lin, Y., \& Yang, G. S. (2012). A driverautomation system for brake assistance in intelligent vehicles. IEEE 10th International Conference on Industrial Informatics, 446-451. https://doi.org/10.1109/ INDIN.2012.6301063

Mostafa, S. A., Mustapha, A., Hazeem, A. A., Khaleefah, S. H., \& Mohammed, M. A. (2018). An Agent-Based Inference Engine for Efficient and Reliable Automated Car Failure Diagnosis Assistance. IEEE Access, 6, 8322-8331. https:// doi.org/10.1109/ACGESS.2018.2803051

Niehaus, A., \& Stengel, R. F. (1991). An expert system for automated highway driving. IEEE Control Systems Magazine, 11, 53-61. https://doi.org/10.1109/37.75579 
Wang, F.-Y., Zeng, D., \& Yang, L. (2006). Smart cars on smart roads: an IEEE intelligent transportation systems society update. IEEE Pervasive Computing, 5(4), 68-69. https:// doi.org/10.1109/MPRV.2006.84 


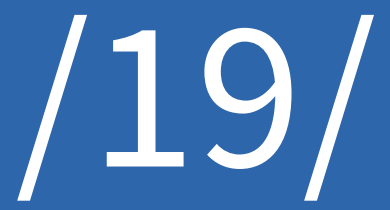




\title{
IMPLEMENTATION OF DIFFERENTIAL EVOLUTION ALGORITHM TO PERFORM IMAGE FUSION FOR IDENTIFYING BRAIN TUMOR
}

\author{
Pothiraj Sivakumar \\ Department of Electronics and Communication Engineering, \\ Kalasalingam Academy of Research and Education, \\ Virudhunagar District, Tamil Nadu, (India). \\ E-mail: siva@klu.ac.in ORCID: https://orcid.org/0000-0003-1328-8093
}

Subbiah Parvathy Velmurugan

Department of Electronics and Communication Engineering,

Kalasalingam Academy of Research and Education,

Virudhunagar District, Tamil Nadu, (India).

E-mail: s.p.velmurugan@klu.ac.in ORCID: https://orcid.org/0000-0002-3314-1454

Jenyfal Sampson

Department of Electronics and Communication Engineering, Kalasalingam Academy of Research and Education,

Virudhunagar District, Tamil Nadu, (India). E-mail: jenyfal.sampson@klu.ac.in ORCID: https://orcid.org/0000-0001-8007-3995

Recepción: 05/12/2019 Aceptación: 30/12/2019 Publicación: 23/03/2020

\section{Citación sugerida:}

Sivakumar, P., Velmurugan, S. P., y Sampson, J. (2020). Implementation of differential evolution algorithm to perform image fusion for identifying brain tumor. 3C Tecnología. Glosas de innovación aplicadas a la pyme. Edición Especial, Marzo 2020, 301-311. http://doi.org/10.17993/3ctecno.2020. specialissue4.301-311

\section{Suggested citation:}

Sivakumar, P., Velmurugan, S. P., \& Sampson, J. (2020). Implementation of differential evolution algorithm to perform image fusion for identifying brain tumor. 3C Tecnología. Glosas de innovación aplicadas a la pyme. Edición Especial, Marzo 2020, 301-311. http://doi.org/10.17993/3ctecno.2020. specialissue4.301-311 


\section{ABSTRACT}

Automated mechanization for curing a disease is a reliable and protuberant method. A disease in brain can be detected by Magnetic Resonance Imaging (MRI). In this context, image fusion is a method for creating an image by merging pertinent data from 2 or more images. The resultant image will be highly useful than the individual input images to retentive the vital characteristics of every image. Multiple image fusion is a significant method employed in image processing techniques. In this study, differential evolution (DE) algorithm-based image fusion has been performed with MRI and computed tomography (CT) images. The simulation works have been carried out to evaluate the different quality measurements of DE on image fusion.

\section{KEYWORDS}

De-speckling, Brain tumor detection, CT, DE, Image fusion, MRI. 


\section{INTRODUCTION}

Brain tumours is harmful to humans, due to the atypical availability of cells inside the brain. Brain function will be interrupted and be deadly. Benign and malignant tumors are frequently identified. Benign tumors are not as harmful as malignant tumors, because they can grow rapidly. Medical imaging methodologies such as MRI, CT, Ultrasound, $\mathrm{X}$-ray etc. are employed to display the internal body parts for diagnosing (Rowden, 2019). Among them MRI is widely employed and it offers accurate brain images and cancer cells. So, brain tumor can be detected via MRI images. This study concentrates on detection of brain tumor through image fusion. Image fusion is a process of merging two or more images into a single compound image that contains the information of the source images without clamor. Multi-modular recuperative image fusion has been employed to recognize the wounds. In biomedical image processing image fusion has got more attention in the past decade (Daneshvar \& Ghassemian, 2010; Wang, Li, \& Tian, 2014). MRI and CT images held more practical information than biomedical images. The aim of image fusion is to obtain the information at each pixel without damaging the pixel associations of the particular image.

In this context, previously, a complex wavelet modification for image fusion has been proposed to attain the optimal combination using Lifting wavelet transform (LWT), Multiwavelet transform (MWT), Stationary wavelet transform (SWT) and spatial domain (Principal component analysis (PCA) approaches (Singh \& Khare, 2014). Similarly, undecimated wavelet has been implemented, where the image is crumbled into two successive scrutinizing errands (Ellmauthaler et al., 2013). An affable fusion technique using SWT and NSCT has been presented, where the input image is rotten by SWT and NSCT. The coefficients of SWT and NSGT are combined to form the fused image (Li \& Liu, 2009). A new framework has been proposed where the images considered with SWT primarily and the overall textural topographies have been attained via gray level cooccurrence matrix (Singh \& Khare, 2014; Huang et al., 2014; Shi \& Fang, 2007). Hence, a scheme for fusing MRI and CT images using DE based Debauchee's wavelet Transform (DE-DWT) has been attempted in this study. 


\section{MATERIALS AND METHODS}

As a part of image fusion, pre-handling of images have been performed using DE. DE has been employed to create the fission rubrics. The preprocessing steps involved in image fusion have been illustrated in Figure 1.

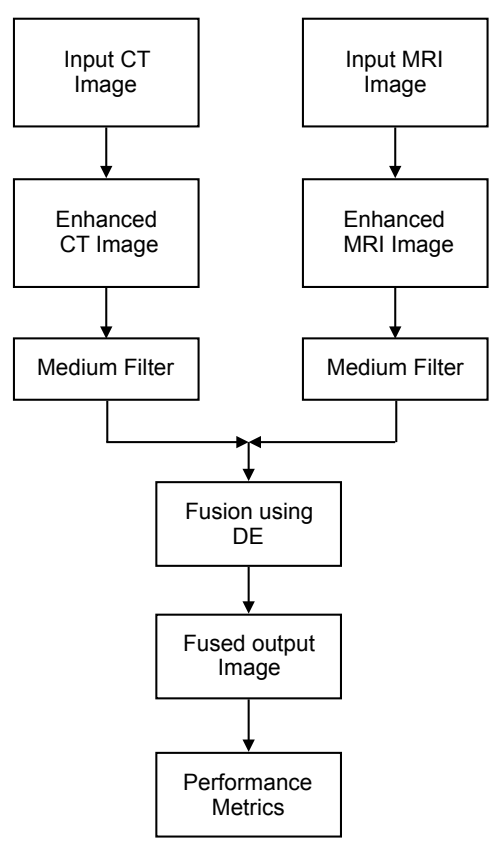

Figure 1. Flowchart of proposed approach.

Primarily, the informative source images such as CT and MRI images have been collected. Subsequently, the source images have been converted into dark scale and resized. The enhancement of quality of the images has been performed using imadjust order available in MATLAB simulation. Commotion dismissal has been carried out by using median channel. This is an excellent method in ejecting salt and pepper commotions of biomedical images. It happens due to the movement of antiquities.

Performance indices such as Mean Square Error (MSE), Peak Signal to Noise Ratio (PSNR), contrast and homogeneity have been estimated. The amount of clamor available in the image is denoted as PSNR. It is used to indicate the obtained fused image has tumbleddown or not. MSE value need to be low and PSNR value need to be high. 


$$
\begin{gathered}
M S E=\frac{1}{m n} \sum_{i=0}^{m-1} \sum_{j=0}^{n-1}[(i, j)-K(i, j)]^{2} \\
P S N R=20 \log _{10}\left(\frac{M A X}{\sqrt{M S E}}\right)
\end{gathered}
$$

Contrast reinstates the data associated with the pixel with the adjacent pixel. It has been calculated as follows.

$$
\text { Contrast }=\sum(i-j) 2 \times P(i, j)
$$

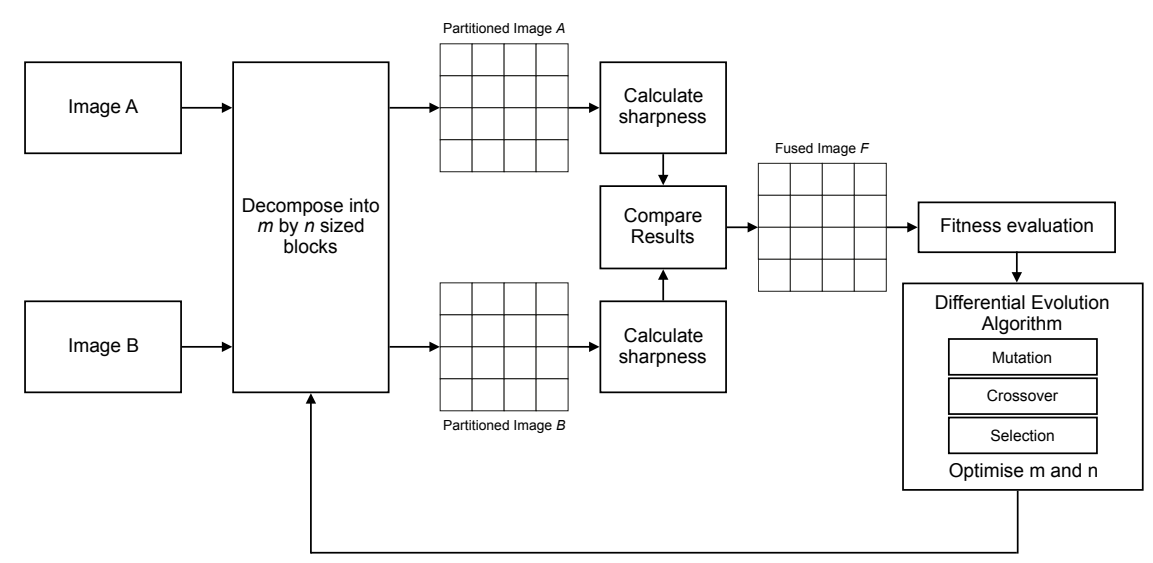

Figure 2. Flowchart for image fusion using DE.

Table 1. Best parameters of DE.

\begin{tabular}{|c|c|}
\hline Parameter & Value \\
\hline Number of population & 100 \\
\hline Maximum generation & 100 \\
\hline Crossover probability & 0.5 \\
\hline Scaling factor & 0.9 \\
\hline
\end{tabular}

Homogeneity has been used to estimate the intimacy of components availed in gray level concurrence matrix (GLCM).

\section{DIFFERENTIAL EVOLUTION ALGORITHM}

Price and Storn introduced DE as a population-based stochastic direct search technique. The implementation procedure of DE has been adopted from Aslantas and Toprak (2014). 
The steps involved in DE based image fusion have been illustrated in Figure 2. The best control parameters for DE have been provided in Table 1 .

The performance indices such as MSE, PSNR, Contrast, Entropy and Homogeneity have been presented in Table 2 .

Table 2. Performance indices of $D E$ on image fusion.

\begin{tabular}{|c|c|c|c|c|c|}
\hline SET & MSE & PSNR & Entropy & Contrast & Homogeneity \\
\hline 1 & 12447 & 5.4875 & 10.1457 & 0 & 1 \\
\hline 2 & 10253 & 6.0248 & 11.2488 & 0 & 1 \\
\hline 3 & 17305 & 8.1027 & 12.5761 & 0 & 1 \\
\hline
\end{tabular}

\section{RESULTS AND DISCUSSIONS}

MRI and CT images have been fused together using DE. The ultimate objective of image fusion is to acme the valuable data from various input images. The adaptive fuzzy clustering rule has been employed to fragment the region of interest (ROI) to isolate the tumor from the resultant fused image. It will group the various grade intensity segments of the fused image. The segments with huge grade intensity are marked as the tumor, and they have been isolated using thresholding method (Chabira, Skanderi, \& Aichouche, 2013).

Figures 3 (a), (b), (c) and (d) provide the information about the CT and MRI images which are processed for fusion. Figures 3 (a) and (b) displays the gray scale CT and MRI images respectively. Figures 3 (c) and (d) demonstrate the median filtered CT and MRI images respectively. DE-DWT has been involved in the fusing mechanism. Using the fusing rules, fusing rules, the input images have been combined. Diverse levels have been fixed to decide clamor data adversity in the image. Figures 4 (a) and (b) demonstrate decomposed CT and MRI images. Figure 5 illustrates the resultant fused image with decent idiosyncratic enrichment. By following the DE-DST rule, least value of CT is combined with the least level decomposed MRI image to form the fused image. 


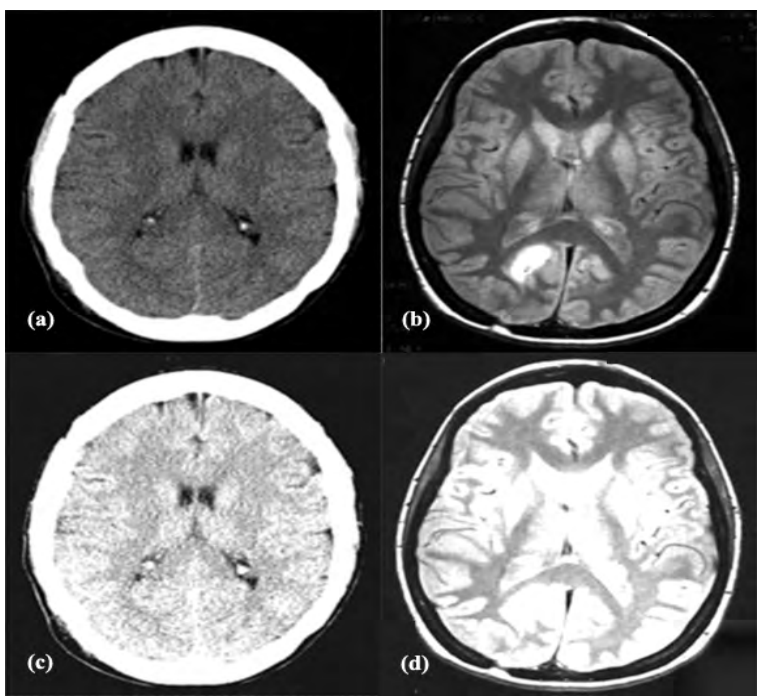

Figure 3. (a) Gray scale CT image (b) Gray scale MRI image (c) Median filtered CT image (d) Median filtered MRI images.

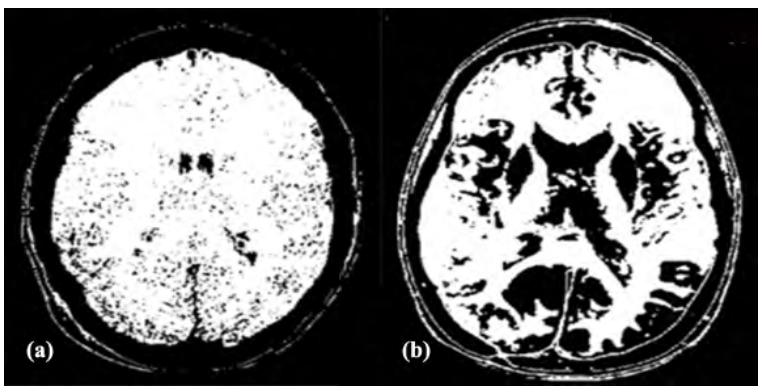

Figure 4. (a) Decomposed CT image (b) Decomposed MRI image.

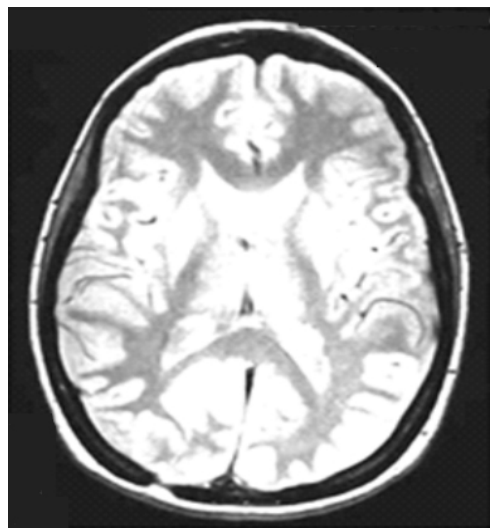

Figure 5. Resultant fused image. 


\section{CONCLUSION}

A typical muscles grown in brain disturb brain activities and that is referred as brain tumor. Biomedical image processing aims to recognize precise data through images with minimum error. Detection of brain tumor via MRI images is not easy due to the intricacy of brain. A pixel based image fusion procedure using DE-DWT has been proposed in this study. The simulations have been carried out with CT and MRI images. The performance indices such as entropy, MSE, PSNR, contrast and homogeneity imply the effectiveness of the proposed DE-DWT approach. 


\section{REFERENCES}

Aslantas, V., \& Toprak, A. N. (2014). Multi focus image fusion by differential evolution algorithm. In Proceedings of the 11th International Conference on Informatics in Control, Automation and Robotics - Volume 2: ICINCO, 312-317, Vienna, Austria. https://doi. $\mathrm{org} / 10.5220 / 0005061103120317$

Chabira, B., Skanderi, T., \& Aichouche, B. A. (2013). Unsupervised change detection from multitemporal multichannel SAR images based on stationary wavelet transform. In 7th International Workshop on the Analysis of Multi-temporal Remote Sensing Images (Multi-Temp). https://doi.org/10.1109/Multi-Temp.2013.6866025

Daneshvar, S., \& Ghassemian, H. (2010). MRI and PET image fusion by combining IHS and retina-inspired models. Information Fusion, 11(2), 114-123. https://doi. org/10.1016/j.inffus.2009.05.003

Ellmauthaler, A., Pagliari, G. L., \& da Silva, E. A. B. (2013). Multiscale Image Fusion Using the Undecimated Wavelet Transform With Spectral Factorization and Nonorthogonal Filter Banks. IEEE Transactions on Image Processing, 22(3), 1005-1017. https://doi.org/10.1109/TIP.2012.2226045

Huang, P. W., Ghen, G. I., Lin, P. L., Ping, G., \& Hsu, L. P. (2014). PET and MRI brain image fusion using wavelet transform with structural information adjustment and spectral information patching. In 2014 IEEE International Symposium on Bioelectronics and Bioinformatics (IEEE ISBB 2014), 1-4. https://doi.org/10.1 109/isbb.2014.6820901

Li, Y., \& Liu, G. (2009). Cooperative Fusion of Stationary Wavelet Transform and Nonsubsampled Contourlet for Multifocus Images. In Second International Symposium on Computational Intelligence and Design, 1, 314-317. https://doi.org/10.1109/ ISCID.2009.86

Rowden, A. (2019). Types, symptoms, and treatment of a brain tumor. https://www. medicalnewstoday.com/articles/315625.php

Sahoo, T., \& Patnaik, S. (2008). Cloud Removal from Satellite Images Using Auto Associative Neural Network and Stationary Wevlet Transform. In First International 
Conference on Emerging Trends in Engineering and Technology, 100-105, Nagpur, Maharashtra, India. IEEE. https://doi.org/10.1109/ICETET.2008.99

Shi, H., \& Fang, M. (2007). Multi-focus Color Image Fusion Based on SWT and IHS. In Fourth International Conference on Fuzzy Systems and Knowledge Discovery (FSKD 2007), 2, 461-465. https://doi.org/10.1109/FSKD.2007.414

Singh, R., \& Khare, A. (2014). Fusion of multimodal medical images using Daubechies complex wavelet transform - A multiresolution approach. Information Fusion, 19, 4960. https://doi.org/10.1016/j.inffus.2012.09.005

Wang, L., Li, B., \& Tian, L. F. (2014). Multi-modal medical image fusion using the interscale and intra-scale dependencies between image shift-invariant shearlet coefficients. Information Fusion, 19, 20-28. https:/ / doi.org/10.1016/j.inffus.2012.03.002 


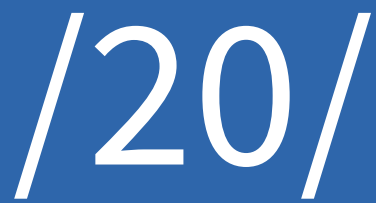




\section{OPTIMAL CHOICE OF SUPERVISED TECHNIQUES FOR MR IMAGE CLASSIFICATION}

Balasubramanian Aruna Devi

Electronics and Communications engg, Kalasalingam Academy of research and Education, Krishnankoil, (India). E-mail: b.arunadevi@klu.ac.in ORCID: https://orcid.org/0000-0002-0981-804X

\section{Murugan Pallikonda Rajasekaran}

Professor. Electronics and Communications engg, Kalasalingam Academy of research and Education

Krishnankoil, (India). E-mail: m.p.raja@klu.ac.in ORCID: https://orcid.org/0000-0001-6942-4512

\section{Citación sugerida:}

Devi, B. A., y Rajasekaran, M. P. (2020). Optimal choice of supervised techniques for MR image classification. 3C Tecnología. Glosas de innovación aplicadas a la pyme. Edición Especial, Marzo 2020, 313-327. http://doi.org/10.17993/3ctecno.2020.specialissue4.313-327

\section{Suggested citation:}

Devi, B. A., \& Rajasekaran, M. P. (2020). Optimal choice of supervised techniques for MR image classification. 3C Tecnología. Glosas de innovación aplicadas a la pyme. Edición Especial, Marzo 2020, 313-327. http://doi.org/10.17993/3ctecno.2020.specialissue4.313-327 


\section{ABSTRACT}

Magnetic Resonance Imaging (MRI) is a modern, robust method that uses in the detection of various medical problems. In this research work, a trial is used to attempt for the detection of tumour in pancreas MR images. An automated classifier is used for detection of tumour in MR images and avoids the drawbacks of MRI. This automated classifiers can detect automatically, either the MR image is affected or not affected. Features are extracted from MR images using second order statistics approach and are classified by two techniques Support Vector Machine (SVM) and Extreme Learning Machine (ELM). SVM approach has high classification accuracy $(96 \%)$ which is higher than ELM, while ELM performs faster compared to SVM.

\section{KEYWORDS}

SVM, ELM, GLCM feature extraction, Image classification. 


\section{INTRODUCTION}

In medicine field, medical Image analysis and processing has enormous applications. It has emerged as one of the superior tools to diagnose as well as detect many disorders. It permits both radiologists and doctors to make exact detection, by analyzing and visualizing the medical image. Computer-Aided Diagnosis (CAD) is an approach that is achieving attention in modern life. It can comfort doctors accurately read images and diagnosis possible decisions to avoid incorrect understanding of lesions. It is necessary to mark that CAD systems can only present a second opinion and can by no means follow physicians or radiologists. There are many imaging modalities for the humans of tissue analysis, such as Magnetic Resonance Imaging (MRI), mammogram function, Computed Tomography (CT) and so on. The main target of this research work is on MRI images. MRI (Armstrong, Cohen, Weinbrg, \& Gilbert, 2004) is a medical imaging method that generates images of the inner part of human body. It is an on-radio active, non-aggressive, pain-free method for visualizing detailed data regarding the normal or tumors without any human involvement. The target of this research work is to grant an automatic detection tool that will guide physicians or radiologists in detecting lesions by diagnosing them from normal tissue. The first step is to extract the features in MRI Images by second order statistics. These extract important image features from the MR Images are used to classify the image is affected or not affected. This will help the physicians or radiologists in the analysis of diagnosing tumor in MR images. In this research work, we have analyzed two classifiers such as SVM (Vapnik, 1995) \& ELM (Huang, Zhu, \& Siew, 2004).

\section{SVM}

SVM is a classification approach for high-dimensional data which is presented by Vapnik (1995) to resolve the discrimination disputes of two issues. SVM has been broadly used in the fields of medical image processing, image retrieval, text analysis, and so on. SVM is based on the working principle that the data in the input space can be linear dividable in a higher dimensional feature space after a certain mapping. 


\section{ELM}

ELM (Huang et al., 2004) is a newly advanced machine learning approach, extensively applied in image processing, computer Vision, biomedical applications, system modeling and regression.

The first stage describes with the feature extraction applying GLCM in MRI pancreas images. In the second stage, they are discriminated by the classification methods such as SVM \& ELM. The output displayed that SVM classification method has the superior classification accuracy compared to ELM.

\section{LITERATURE SURVEY}

Lu et al. (2017) presented DWT feature extraction and classified by bat algorithm based extreme learning machine with a classification accuracy of 93.33\%. Nazir, Wahid, and Khan (2015) classified brain MRI using various moment features extraction and artificial neural networks with an accuracy of 94.24\%. Nandpuru, Salankar, and Bora (2014) classified brain tumor applying Texture features, symmetrical and gray features extraction, principal component analysis (PCA) feature selection and support vector machine (SVM) classification. The classification accuracy was 84\%. Ibrahim, Osman, and Mohamed (2018) classified MR brain images using wavelet-based features extraction, features selection by PGA, and classified by artificial neural networks (ANN) with a classification accuracy of 96.33\%. Othman, Abdullah, and Kamal (2011) discriminated normal and abnormal MRIs using DWT feature extraction, principal component analysis (PCA) feature selection and SVM classification by 65\% classification accuracy. Kavitha and Thyagharajan (2012) have presented histogram, textural features and classified by SVM with an accuracy of 90\%. Diz, Marreiros and Freitas (2015) have described GLCM and Grey-Level Run Length Matrix (GLRLM) feature extraction for mammogram image classification and achieved 76\% accuracy. Dheeba and Selvi (2011) have presented Laws texture features to discriminate images into Benign and Malignant (MIAS-Mammographic Image Analysis Society database) and gained 86.10\% accuracy. Shah, Surve and Turkar (2015) classified pancreatic tumor of CT images using Minimum distance classifier. The classification accuracy was 61.59\%. Yao, Chen and Chow (2009) described wavelet transform features extraction method and classified by SVM with an accuracy of $83 \%$. Aruna Devi and Pallikonda 
Rajasekaran (2018) described GLCM features extraction and differentiated normal and abnormal MR images by applying ANN and SVM methods. ANN found that it achieves 96\% classification accuracy. Aruna Devi, Pallikonda Rajasekaran and Thiyagarajan (2019) proposed tumor discrimination by GLCM feature extraction, JAFER feature selection and comparison among 5 types of classification modes, ANN BP gains 98\% classification accuracy. Based on the survey, the discrimination capability of classifiers is very less and computational time is also high. Our proposed technique increases the discrimination capability as high $(98 \%)$ that is superior to previous research analysis. In this research work, we are going to discriminate the $160 \mathrm{MR}$ images by affected or not affected using second order statistics feature extraction and discriminated by two modes such as ANN and SVM. Sensitivity, specificity and classification accuracy is measured and compared among the two modes.

\section{MATERIALS AND METHODS}

Input data set:

The dataset used for predicting the performance of the proposed model in this research work is based on the MR pancreas medical images that are gathered from the health care centres. The numbers of medical pancreas images totally 160 of which 100 are normal and 60 are abnormal images. Figure 1 shows the normal pancreas images and Figure 2 shows the abnormal pancreas images. Figure 3 displays the flowchart.

\section{FEATURES EXTRACTION}

Features extraction by second order statistics:

The procedure of transferring the input image into a set of features is known as feature extraction. Features normally consist of data relevant to colour, shape, texture or context. First order statistics provides gray level pixels occur in an image. First order measures are mean, variance, skewness and kurtosis. Second order statistics provides inter relationship between pixel and its neighbors. They provide detailed information about the pixel and its neighbors with an angle of 0, 45, 90 and 135 degrees at a distance d. Second order measures are entropy, energy, contrast, homogeneity, sum of variance, cluster prominence, 
sum of entropy, cluster shade, information measure of correlation. Second order statistics features are tabulated in Table 1. In this work, these features are measured and classified by two classifiers namely SVM and ELM.

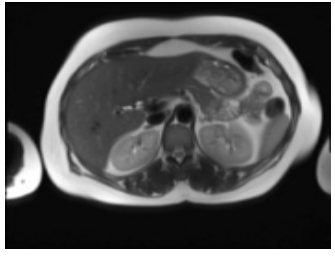

a

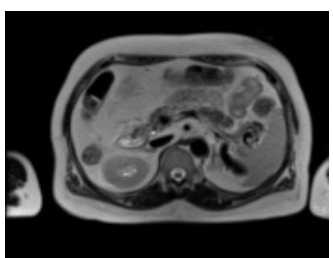

d

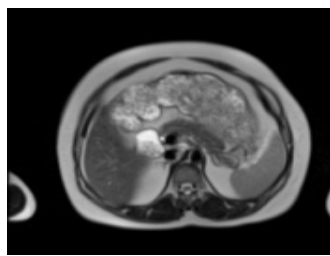

g

Figure 1. Normal pancreas MR images.

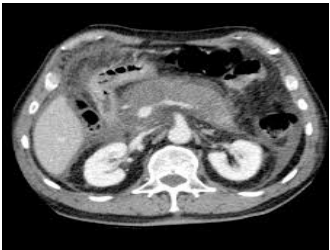

a

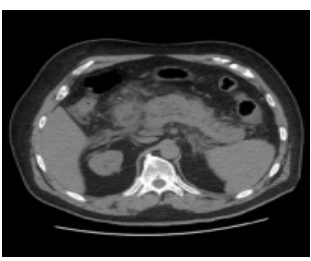

d

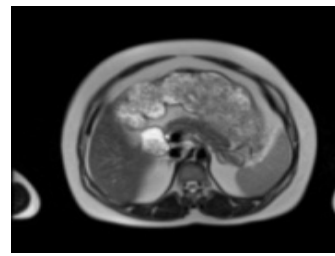

b

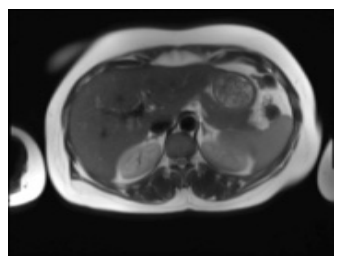

e

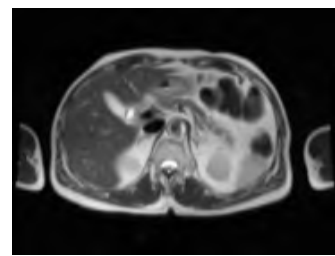

h

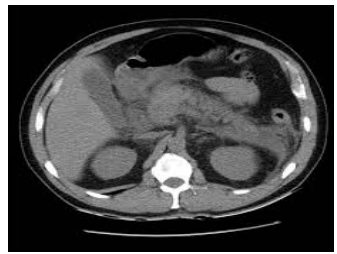

b

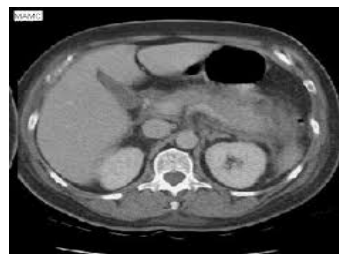

d

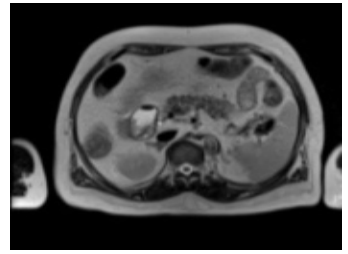

C

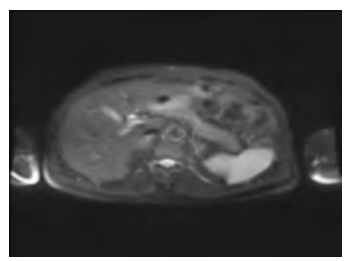

f

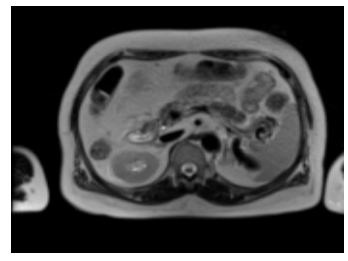

i

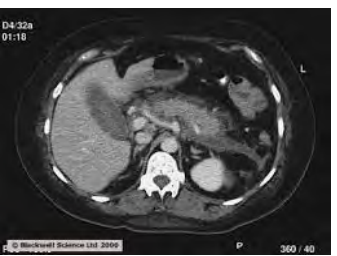

c

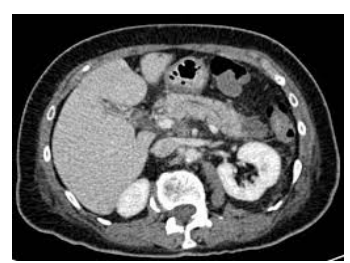

$\mathrm{f}$ 


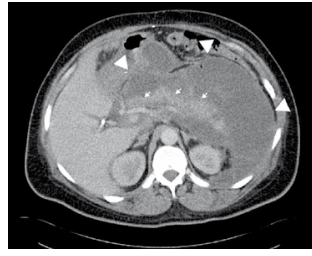

g

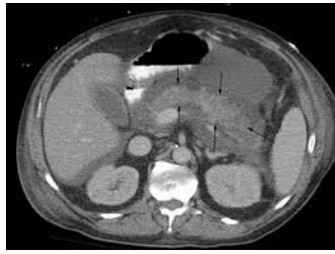

h

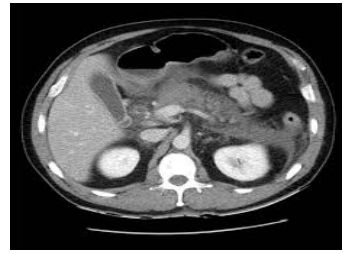

i

Figure 2. Abnormal pancreas MR images.

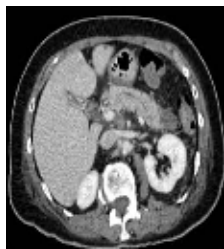

Features extraction using second order statistics

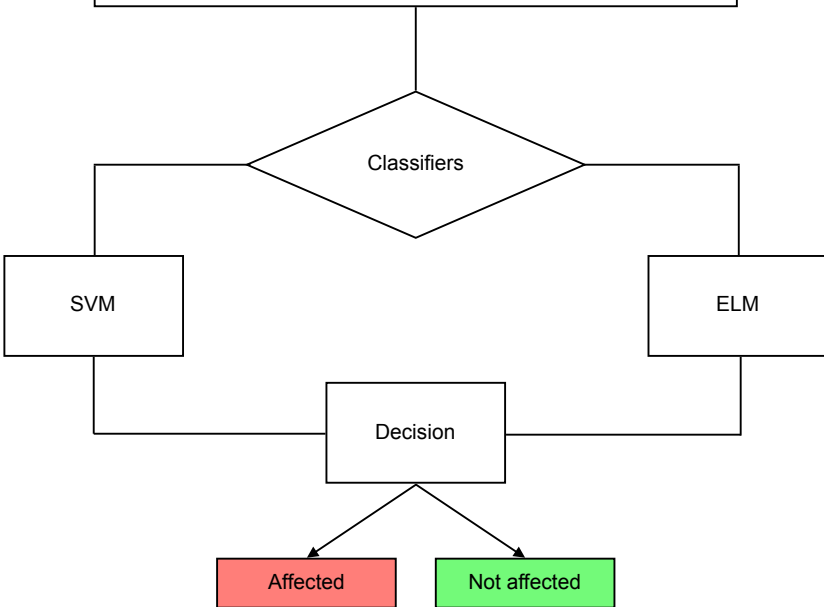

Figure 3. Flow chart.

Table 1. GLCM features.

$$
\begin{aligned}
& \text { GLCM features: } \\
& \text { Contrast: } \quad \sum_{i} \sum_{j}(i-j)^{2} g_{i j} \\
& \sum_{i} \sum_{j}(i-j)^{2} g_{i j}(1) \sum_{i} \sum_{j}(i-j)^{2} g_{i j} \sum_{i} \sum_{j}(i-j)^{2} g_{i j} \sum_{i} \sum_{j}(i-j)^{2} g_{i j} \\
& \text { Energy: } \quad \sum_{i} \sum_{j} g_{i j} \sum_{i} \sum_{j} g_{i j^{2}} \\
& \text { Entropy: } \quad \sum_{i} \sum_{j} g_{i j} g_{i j} \sum_{i} \sum_{j} g_{i j} g_{i j} \\
& \sum_{i} \sum_{j}(i-j)^{2} g_{i j} \sum_{i} \sum_{j}(i-j)^{2} g_{i j} \sum_{i} \sum_{j} g_{i j} g_{i j} \\
& \text { Homogeneity: } \left.\left.\quad \sum_{i} \sum_{j} \frac{(1}{1+(i-j)^{2}}\right) g_{i j}: \sum_{i} \sum_{j} \frac{(1}{1+(i-j)^{2}}\right) g_{i j} \\
& \left.\sum_{i} \sum_{j} \frac{(1}{1+(i-j)^{2}}\right) g_{i j}
\end{aligned}
$$




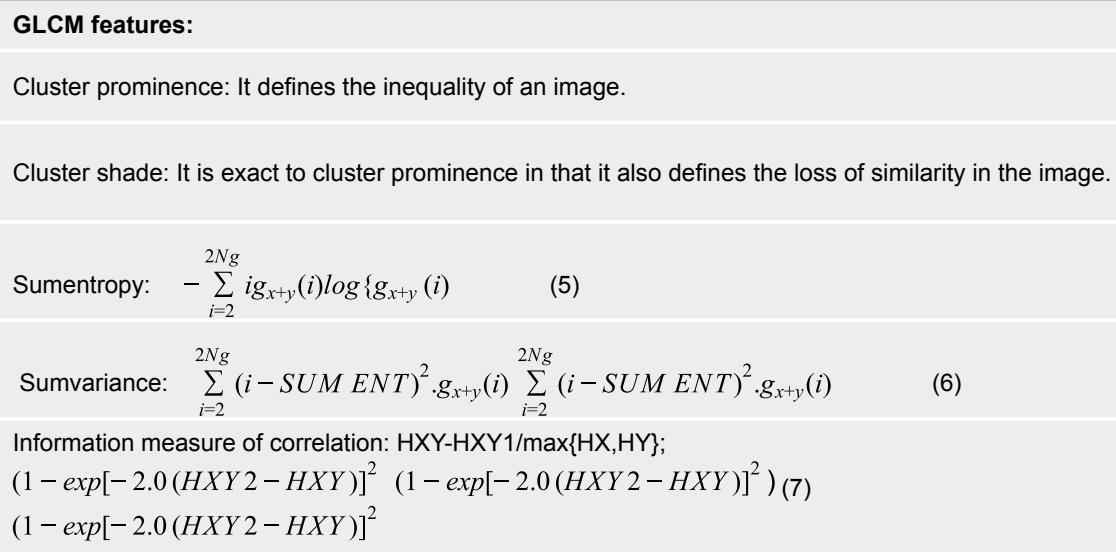

\section{CLASSIFICATION}

Classification is the procedure of classifying a known input by a good classifier. The main target of proper classification is to provide a label to each MR image based on second order statistics features.

\section{SVM}

SVM is used for mapping complex feature space into linear feature space. It works on the base of fitting a boundary to a field of points that are belongs to one class with one another. Once boundary is fixed, on the learned samples, for any unknown points that are test sample need to be classified, and the accuracy will be predicted. Once boundary is fixed, maximum training points are redundant. All it demands a group of points that can identify and fits the boundary. The group of points are known as support vectors and the boundary is called as hyperplane.

\section{ELM}

Extreme Learning Machine (ELM) is a single hidden-layer feed-forward neural network (SLFN). The significance of the SLFN should be convenient for information such as weight, threshold value, and activation function so that superior training can be achieved. In gradient-based learning, all of these quality measures are changed iteratively for the significant value. Therefore, due to the possibility of being attached to the slow and local minimum, the performance can generate low outputs. On the basis of the gradient in the ELM training process, the output weights are analytically calculated where the input 
weights are chosen randomly. In this process, the success rate raises because the resolution time and the errors can actively shorten the possibility of being fitted to a local minimum.

\section{TRAINING AND TESTING}

Feature dataset is classified by the classification such as SVM \& ELM techniques. Out of 165 pancreas MR images, $70 \%$ Images were used for learning and $30 \%$ of images were used for testing.

There are more classification techniques, where training dataset are favoured by random sampling with restoration for MR pancreas image classification. Basically, choosing the best feature election approach with its performance parameters, it was required for process of classification approaches. In continuity, the classification outcomes with parameters for each discrimination approach are followed. Performance parameters were employed for each discrimination approach and the best one was choose for tumour identification.

Support vector machine is relevant for high dimension small-sample learning and nonlinear problems. SVM mainly focus at binary classification. SVM provides strong generalization ability and structural risk minimization. It separates the two classes using hyper plane. The second order statistics features are used as input of the classifier and the corresponding known label (that is affected or not affected) is the output of the classifier. The hyper plane separates the class as affected or not affected. It helps to train the classifier as input, output mappings functions. It provides the superior minimum distance to the learning data. The SVM has many kernel functions. The predominant kernel function is RBF that denotes radial basis function. Here RBF kernel is used. After learning the classifier, the test set was applied to test the significance of the classifier and its capability to accurately discriminate the MR images as either affected or not affected. To check out our SVM classifier, a confusion matrix was generated as shown in Figure 4 and the classification accuracy is $96 \%$ which is tabulated in Table 2.

\section{ELM}

Conventional single hidden-layer feed forward neural networks (SLFNs), such as the back propagation (BP) method, have been applied for research in many applications. The weight which assigns the hidden nodes is applied randomly and weights are not altered forever. 
The weights that correlate hidden nodes with outputs are trained in one step. ELM yields cooperative training technique. Even though ELM confirms to be terribly quick and in a great way in observation, it has some drawbacks. The election of hidden neurons is placed on trial and error technique that can provide into inadequate results. ELM provides 89\% classification accuracy.

Next, the performance evaluation approaches applied to test the two methods (SVM \& ELM). We analyse the execution of the methods in terms of sensitivity, specificity and accuracy.

\section{Performance opinion parameters:}

$$
\begin{gathered}
\text { Sensitivity }=T P /(T P+F N) 100 \% \\
\text { Specificity }=T N /(T N+F P) 100 \% \\
\text { Accuracy }=(T P+T N) /(T P+T N+F P+F N) 100 \%
\end{gathered}
$$

Where:

TN $($ True Negative $)=$ perfectly discriminated negative cases, $\mathrm{TP}($ True Positives $)=$ perfectly discriminated positive cases, FN (False Negative) = imperfectly discriminated positive cases FP (False Positives $)=$ imperfectly discriminated negative cases.

Specificity measures how perform the system can predict the negatives. Sensitivity is the rate of perfectly discriminated positives, describes best performance of the approach in predicting positives. Accuracy confirms the whole correctness of the classifier in predicting both positive and negative cases in terms of tumor.

\section{RESULTS}

SVM and ELM are learned by second order statistics features and classify the pancreas MR image as affected or not affected (normal or abnormal). SVM method provides good accuracy than ELM. The accuracy of SVM method is $96 \%$, specificity and sensitivity are $95 \%$ and $97 \%$ respectively. The classification accuracy of ELM is $89 \%$, specificity and sensitivity are 95\%and 92\% respectively. Table 2 represents classification accuracy, sensitivity and specificity for two methods. Figure 4 represents SVM confusion matrix. Figure 5 represents ELM ROC curve is graphed as a plot of true-positive rate on the y-axis and false-positive rate on the $\mathrm{x}$-axis. 


\section{CONCLUSION}

Among the two methods, SVM is the superior method which gives $96 \%$ classification accuracy. It proves that our proposed SVM technique gives high accuracy of $96 \%$, which is compared to other previous techniques. In this work, using second order stastics features are extracted and classified by SVM and ELM. Results are compared and proved SVM provides high classification accuracy of $96 \%$. In future, best features are selected using feature reduction methods (forward selection, backward elimination) and are classified by SVM and other classifiers to realize which classifier is best in practice.

\section{FUTURE WORK}

In this work, using second order statistics features are extracted and classified by SVM and ELM. Results are compared and proved SVM provides high classification accuracy of $96 \%$. In future, best features are selected using feature reduction methods (forward selection, backward elimination) and are classified by SVM and other classifiers to realize which classifier is best in practice.

Table 2. Comparative analysis of SVM and ELM techniques.

\begin{tabular}{|c|c|c|c|c|}
\hline $\begin{array}{c}\text { Classification } \\
\text { Techniques }\end{array}$ & $\begin{array}{c}\text { Classification } \\
\text { accuracy }\end{array}$ & Sensitivity & Specificity & Time \\
\hline SVM & 96.67 & 97.3 & 95.65 & 1.020 s \\
\hline ELM & 89.05 & 92.3 & 95.24 & 0.320 s \\
\hline
\end{tabular}

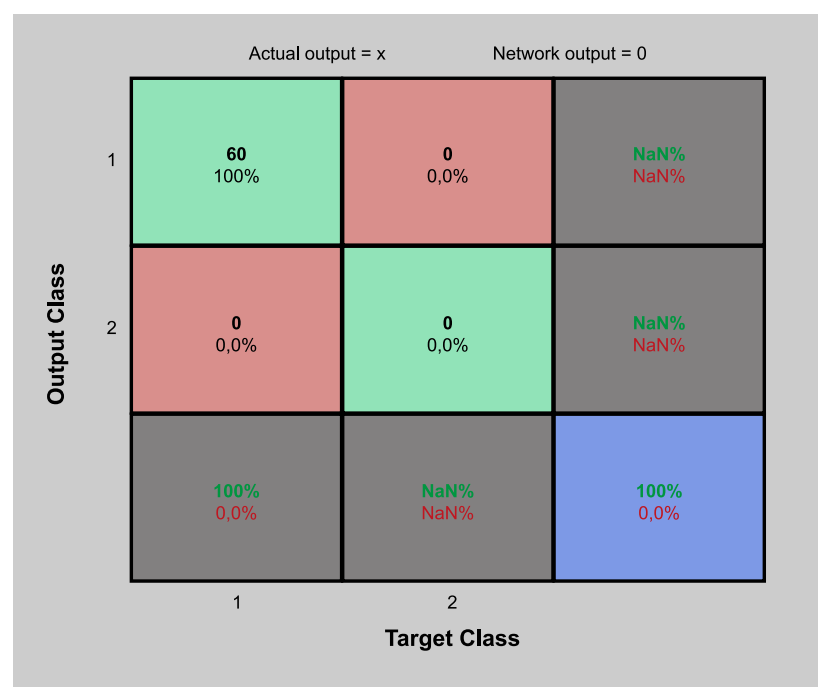

Figure 4. SVM confusion matrix. 


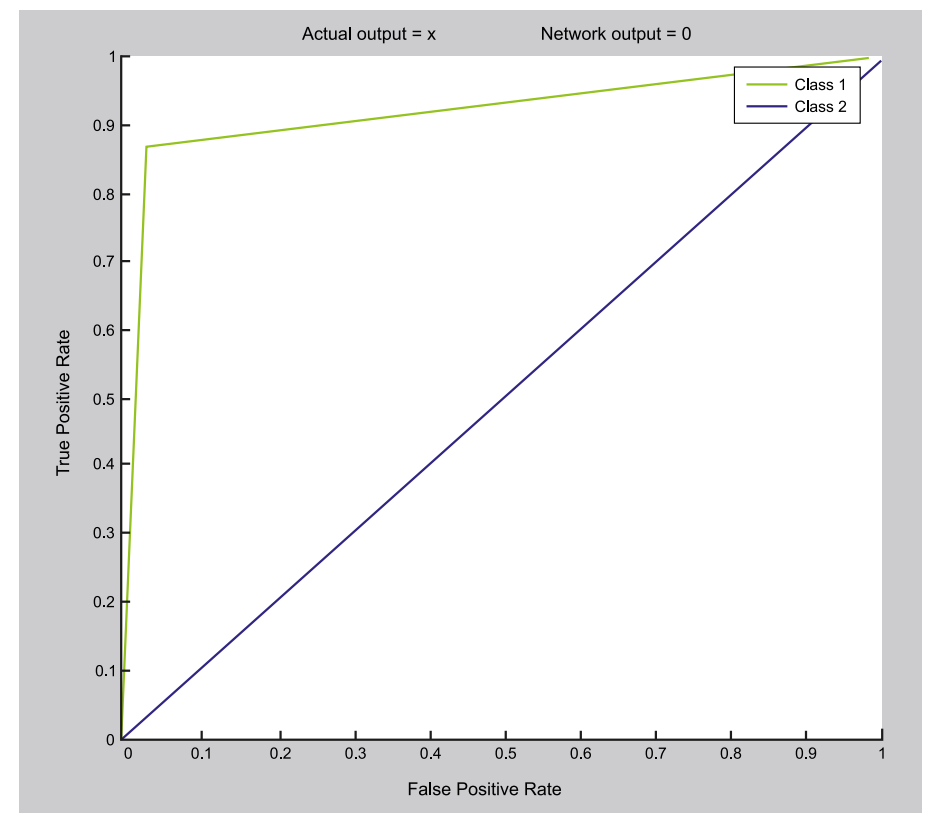

Figure 5. ELM ROC.

\section{ACKNOWLEDGEMENT}

The authors thank the management of Kalasalingam Academy of research and education for granting financial assistance underside the rule of University Research Fellowship (URF) in Department of Electronics and Communication Engineering. Also, we thank KGS health care centre, Madurai for granting the pancreas MR image that is very useful for this research work 


\section{REFERENCES}

Armstrong, T. S., Cohen, M. Z., Weinbrg, J., \& Gilbert, M. R. (2004). Imaging techniques in neuro oncology. Seminars in Oncology Nursing, 20(4), 231-239. https:// doi.org/10.1016/j.soncn.2004.07.003

Aruna Devi, B., \& Pallikonda Rajasekaran, M. (2018). Performance Evaluation of MRI Pancreas Image Classification Using Artificial Neural Network (ANN). In Satapathy S., Bhateja V., Das S. (eds.) Smart Intelligent Computing and Applications. Smart Innovation, Systems and Technologies, vol. 104. Springer, Singapore. https://doi. org/10.1007/978-981-13-1921-1_65

Aruna Devi, B., Pallikonda Rajasekaran, M., \& Thiyagarajan, A. P. (2019). Analysis and classification of malignancy in pancreatic magnetic resonance images using neural network techniques. International journal of imaging systems and technology. https:/ / doi.org/10.1002/ima.22314

Huang, G., Zhu, Q., \& Siew, G. (2004). Extreme learning machine: a new learning scheme of feedforward neural networks. In 2004 IEEE International foint Conference on Neural Networks (IEEE Cat. No.04CH37541), 2, 985-990, vol. 2. https://doi.org/10.1109/ IJCNN.2004.1380068

Ibrahim, W. H., Osman, A. A., \& Mohamed, Y. I. (2013). MRI brain image classification using neural networks. In 2013 International Conference on Computing, Electrical and Electronic Engineering (ICCEEE), 253-258. https://doi.org/10.1109/ ICCEEE.2013.6633943

Kavitha, S., \& Thyagharajan, K. K. (2012). Features based mammogram image classification using weighted feature support vector machine. In Krishna P. V., Babu M. R., Ariwa E. (eds.) Global Trends in Information Systems and Software Applications. ObCom 2011. Communications in Computer and Information Science, vol. 270. Springer, Berlin, Heidelberg. https://doi.org/10.1007/978-3-642-29216-3_35 
Lu, S. S., Qiu, X., Shi, J., Li, N., Lu, Z.H., Chen, P., ... Zhang, Y. D. (2017). A pathological brain detection system based on extreme learning machine optimized by bat algorithm. CNS \& neurological disorders drug targets, 16(1), 23-29. https://doi.org /10.2174/1871527315666161019153259

Nandpuru, H. B., Salankar, S. S., \& Bora, V. R. (2014). MRI brain cancer classification using Support Vector Machine. In 2014 IEEE Students' Conference on Electrical, Electronics and Computer Science, 1-6. https://doi.org/10.1109/SCEECS.2014.6804439

Nazir, M., Wahid, F., \& Khan, S. A. (2015). A simple and intelligent approach for brain MRI classification. Fournal of Intelligent and Fuzzy Systems, 28, 1127-1135. https://doi. org/10.3233/IFS-141396

Othman, M. F., Abdullah, N., \& Kamal, N. F. (2011). MRI brain classification using support vector machine. In 2011 Fourth International Conference on Modeling, Simulation and Applied Optimization, 1-4. https://doi.org/10.1109/ICMSAO.2011.5775605

Shah, J., Surve, S., \& Turkar, V. (2015). Pancreatic Tumor Detection using Image processing Techniques. Procedia Computer Science, 49, 11-16. https://doi.org/10.1016/j. procs.2015.04.221

Vapnik, V. (1995). The Nature of Statistical Learning Theory. New York: Springer.

Yao,J., Chen,J., \& Ghow, G. (2009). Breast Tumor Analysis in Dynamic Contrast Enhanced MRI Using Texture Features and Wavelet Transform. IEEE Journal of Selected Topics in Signal Processing, 3, 94-100. https://doi.org/10.1109/JSTSP.2008.2011110 


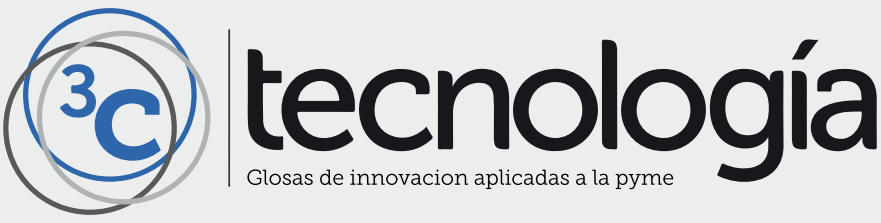

